Estimation of soil water storage change from clay shrinkage using satellite radar interferometry 


\section{Thesis committee}

\section{Promotors}

Prof. Dr S.E.A.T.M. van der Zee

Personal chair Ecohydrology

Wageningen University \& Research

Prof. Dr R.F. Hanssen

Professor of Geodesy and Earth Observation

Delft University of Technology

\section{Co-promotors}

Dr M.J. van der Ploeg

Assistant professor, Soil Physics and Land Management Group

Wageningen University \& Research

Dr G.H. de Rooij

Department Soil Physics, Helmholtz Centre for Environmental Research - UFZ, Halle (Saale), Germany

\section{Other members}

Prof. Dr R. Uijlenhoet, Wageningen University \& Research

Prof. Dr I. Hajnsek, ETH Zürich, Switzerland

Dr F. De Zan, German Aerospace Center, Germany

Dr R.D. Stewart, Virginia Polytechnic Institute and State University, United States of America

This research was conducted under the auspices of the Graduate School for SocioEconomic and Natural Sciences of the Environment (SENSE). 


\title{
Estimation of soil water storage change from clay shrinkage using satellite radar interferometry
}

\author{
Bram te Brake
}

\section{Thesis}

submitted in fulfilment of the requirements for the degree of doctor at Wageningen University

by the authority of the Rector Magnificus

Prof. Dr A.P.J. Mol,

in the presence of the

Thesis Committee appointed by the Academic Board

to be defended in public

on Wednesday 17 May 2017

at 4 p.m. in the Aula. 
Bram te Brake

Estimation of soil water storage change from clay shrinkage using satellite radar interferometry

xvi +126 pages

PhD thesis, Wageningen University, Wageningen, the Netherlands (2017)

With references, with summary in English

ISBN 978-94-6343-163-7

DOI: $10.18174 / 411766$ 


\section{Contents}

$\begin{array}{ll}\text { Abstract } & \text { ix }\end{array}$

Nomenclature $\quad$ xiii

1 Introduction $\quad \mathbf{1}$

1.1 Research background and motivation . . . . . . . . . . . . . . . 1

1.2 Research objectives . . . . . . . . . . . . . . . . . . 3

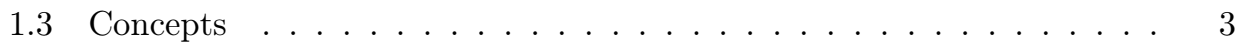

1.3.1 Clay swell and shrinkage . . . . . . . . . . . . . . . 3

1.3.2 Radar interferometry . . . . . . . . . . . . . . . . 5

1.4 Thesis outline . . . . . . . . . . . . . . . . . . . . . . . . . . . . . .

1.5 Prospective audience . . . . . . . . . . . . . . . . 8

2 Radar Interferometry 9

2.1 InSAR principles . . . . . . . . . . . . . . . . . . . . . . 9

2.1.1 Imaging radar observations . . . . . . . . . . . . . . . . . . . . . . . 9

2.1.2 Radar scattering mechanisms . . . . . . . . . . . . . . . 10

2.1.3 Single-Look Complex phase . . . . . . . . . . . . . . . 12

2.1 .4 Interferometric phase . . . . . . . . . . . . . . . . . . . . 13

2.2 Interferometric processing . . . . . . . . . . . . . . . . . . . . 18

2.2.1 Conventional InSAR . . . . . . . . . . . . . . . . . 18

2.2.2 Multi-interferogram techniques ............. 18

3 Shrinkage potential, geometry, and moisture content relations of clay aggregates from a Dutch polder 21

3.1 Introduction . . . . . . . . . . . . . . . . . 21

3.2 Methods . . . . . . . . . . . . . . . . . . . 23

3.2.1 Grain size distribution and clay mineralogy . . . . . . . . . . 23

3.2.2 COLE and PLE . . . . . . . . . . . . . . . . . . . . . . . . . . . . . . . . . 23

3.2.3 Soil shrinkage curve . . . . . . . . . . . . . . . . . . 24

3.3 Results and discussion . . . . . . . . . . . . . . . . . 24

3.3.1 Grain size distribution and clay mineralogy . . . . . . . . . . . 24

3.3.2 COLE and PLE . . . . . . . . . . . . . . . . 25

3.3.3 Soil shrinkage curves . . . . . . . . . . . . . . . . . . . 26

3.3.4 Subsidence modelling . . . . . . . . . . . . . . . 30 
3.4 Conclusions . . . . . . . . . . . . . . . . . . . . . . . . 31

4 Water storage change estimation from in situ shrinkage measurements of clay soils

4.1 Introduction . . . . . . . . . . . . . . . . . . . 33

4.2 Materials and methods . . . . . . . . . . . . . . . . . . . . . 37

4.2.1 Site description . . . . . . . . . . . . . . . . . . . . . . 37

4.2.2 Swelling and shrinkage measurements . . . . . . . . . . . . . . 38

4.2 .3 Volume change . . . . . . . . . . . . . . . . . . . . . . . . 40

4.2.4 Soil moisture measurements . . . . . . . . . . . . . . . . . 40

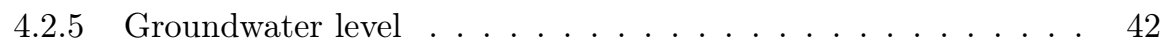

4.2.6 Meteorological data . . . . . . . . . . . . . . . . . . 42

4.3 Results and discussion . . . . . . . . . . . . . . . . . 42

4.3.1 Drying regime, soil shrinkage, and groundwater depth in 2011 . 42

4.3.2 Volume change and soil water storage change of a field soil during extensive drying . . . . . . . . . . . . . . . . . 44

4.3.3 Effects of $r_{\mathrm{s}}$ variation on water storage change of the unsaturated zone . . . . . . . . . . . . . . . . 48

4.3.4 Drying regime, soil shrinkage, and groundwater depth in 2010 . 50

4.3.5 Relation between volume change and soil water storage change in $2010 \ldots \ldots \ldots$. . . . . . . . . . . . . . . . . . 51

4.4 Conclusions and outlook . . . . . . . . . . . . . . . . . . . 55

5 Satellite based radar interferometry to estimate large-scale soil water depletion from clay shrinkage: possibilities and limitations 57

5.1 Introduction . . . . . . . . . . . . . . . . 57

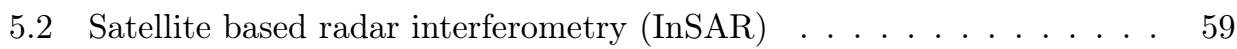

5.2 .1 InSAR measurements of deformation . . . . . . . . . . . . 59

5.2 .2 Interferometric characteristics and limitations . . . . . . . . . 60

5.3 Materials and methods . . . . . . . . . . . . . . . . . . 61

5.3 .1 In situ measurements .................. 61

5.3 .2 TerraSAR-X data. . . . . . . . . . . . . . 62

5.3.3 Information content of phase and in situ observations . . . . . 63

5.4 Results......................... 63

5.4.1 In situ measurements of clay shrinkage . . . . . . . . . . . 63

5.4.2 InSAR observations related to land use, soil type and frost heave 64

5.4.3 Phase observations during the growing season . . . . . . . . . 69

5.4.4 Phase observations of clay shrinkage . . . . . . . . . . . 69

5.5 Discussion . . . . . . . . . . . . . . . . . . . . 72

5.6 Conclusions . . . . . . . . . . . . . . . . . . 75

6 InSAR estimates of clay dynamics related to soil moisture $\quad 77$

6.1 Introduction . . . . . . . . . . . . . . . . . . 77

6.2 Study area, in situ measurements, and radar data . . . . . . . . . . . 78 
6.3 Methodology . . . . . . . . . . . . . . . . . . . . . . 79

6.3.1 Multilooking and coherence estimation . . . . . . . . . . 79

6.3.2 ESM-phase estimation . . . . . . . . . . . . . . . . 81

6.3.3 Spatial phase referencing . . . . . . . . . . . . . . 82

6.3.4 Temporal phase unwrapping. . . . . . . . . . . . . 83

6.4 Soil moisture dielectric phase . . . . . . . . . . . . . . . . . . . 83

6.4.1 Isolation of phase mechanism . . . . . . . . . . . . . . . 83

6.4.2 Soil moisture dielectric phase estimation . . . . . . . . . . . 84

6.5 Shrinkage estimation . . . . . . . . . . . . . . . 85

6.6 Unwrapping correction . . . . . . . . . . . . . . . . . 87

6.6.1 Shrinkage model ................... 87

6.6 .2 Unwrapping results . . . . . . . . . . . . . . . . . . 88

6.7 Shrinkage estimation on all fields in the study area . . . . . . . . . . 89

6.8 Discussion and conclusion . . . . . . . . . . . . . . . . . . . . . . . . . 98

7 Synthesis 101

7.1 Research objectives . . . . . . . . . . . . . . . . . 101

7.1.1 Estimation of soil water storage change from surface elevation changes in clay soils . . . . . . . . . . . . . . . . . 101

7.1.2 Measuring soil surface elevation changes from clay swelling and shrinkage on field to regional scale using satellite based radar interferometry .................. 103

7.1.3 Estimation of soil water storage change from clay shrinkage using satellite radar interferometry . . . . . . . . . . . . 103

7.2 Outlook . . . . . . . . . . . . . . . . . . . . . 104

7.2.1 Further development of the proposed methodology . . . . . . . 104

7.3 Implications for hydrology . . . . . . . . . . . . . . . . . . . 105

7.4 Implications for radar interferometry . . . . . . . . . . . . . . . 106

$\begin{array}{ll}\text { Bibliography } & 107\end{array}$

$\begin{array}{ll}\text { Bedankt! Thanks! } & 119\end{array}$

$\begin{array}{ll}\text { List of publications } & 123\end{array}$ 



\section{Abstract}

Measurements of soil water storage are hard to obtain on scales relevant for water management and policy making. Therefore, this research develops a new measurement methodology for soil water storage estimation in clay containing soils. The proposed methodology relies on the specific property of clay soils to shrink when drying and to swell when (re-)wetted, and the capabilities of a remote sensing technique called satellite based radar interferometry (InSAR) to measure centimetre to millimetre scale displacements of the soil surface. The objective of this thesis was to develop the application of InSAR for soil water storage change estimation on the field scale to regional scale. Two relations are investigated: 1) the relation between water storage change and surface elevation change as a result of swelling and shrinkage of a clayey soil; and 2) the relation between these surface elevation changes and InSAR phase observations.

The shrinkage potential of the soil is very important for successful application of radar interferometry to measure vertical deformation as a result of swelling and shrinkage of clay. Therefore, the shrinkage potential and the water storage change-volume change relation (called the soil shrinkage characteristic, SSC) have been quantified in the laboratory for clay aggregates from the study area in the Purmer, the Netherlands. The clay content of the sampled soil ranged from 3.4 to $23.6 \%$. The aggregates had moderate shrinkage potential over the soil moisture content range from saturation to air-dryness. Shrinkage phases were distinguished based on the portion of water content change that was compensated by volume change. Approximately $40-50 \%$ of water was released in the normal shrinkage phase, where loss of water is fully compensated by volume change. However, the residual shrinkage phase, where volume change is smaller than water content change, started at approx. $50 \%$ normalized soil moisture content (actual moisture content with respect to the moisture content at saturation).

In case of normal shrinkage, soil water storage change can be directly derived from soil volume change. If additionally, clay shrinkage is isotropic, the soil water storage change can be derived from vertical shrinkage measurements. The range of normal and isotropic shrinkage has been assessed in a drying field soil in the study area. To do so, soil water storage change was derived from soil moisture content sensors and groundwater level, and volume change estimates were obtained from soil layer thickness change measurements by ground anchors. Unlike for the aggregates, normal shrinkage was not observed for the field soil, but rather a large degree of linear (basic) shrinkage was observed. In the upper soil layers in the field, normalized soil moisture 
content below $50 \%$ has been observed when drying out. Based on the aggregate SSC, this indicates the occurrence of residual and zero shrinkage in this situation, resulting in less than normal shrinkage when the total unsaturated zone is considered. The water content change - volume change relation thus depends on the scale considered. It was also found that the relation depends on drying intensity, from comparison between shrinkage in a period with prolonged drying and shrinkage in a period with alternating drying end re-wetting.

For the field soil, volume change larger than soil water storage change was observed when assuming isotropic shrinkage. This unrealistic result made clear that the assumption of isotropic shrinkage is invalid. Therefore a correction of the shrinkage geometry factor $r_{\mathrm{s}}$, including dependence of shrinkage geometry on soil moisture content, has been proposed. This correction yielded $r_{\mathrm{s}}$-values between 1.38 and 3 . Dynamics of subsidence porosity (i.e. vertical shrinkage) calculated from the aggregate SSC, and comparison with surface elevation change data from the field study also indicated $r_{\mathrm{s}}$-values smaller than 3 . Values of $r_{\mathrm{s}}$ below 3 , indicate that vertical shrinkage (subsidence) is dominant over horizontal shrinkage (cracking).

Satellite based radar interferometry was applied to measure vertical deformation resulting from clay shrinkage, and evaluate the potential for soil water storage change estimation on the field scale to regional scale. Phase differences between adjacent fields were observed in interferograms over the Purmer area which were hypothesised to be caused by relative motion of the surface level. The combination of a sequence of interferograms covering short time intervals and measurements of soil surface elevation changes in time from ground anchors, indeed revealed similar dynamics in both data. Relative changes between fields in winter were explained by a different effect of frost heave in a bare soil and in a soil permanently covered by grass. Noise in interferograms over agricultural fields was successfully reduced, by multilooking over entire fields. The effect of soil type and land use on phase observation was qualitatively assessed, indicating that agricultural crop fields offer the best phase estimates in winter, while grass fields are more coherent in summer. The results underline the need for careful selection of agricultural fields or areas to base InSAR analysis on.

The differential analysis between fields was extended to time series analysis of phase, to obtain deformation estimates with respect to a stable reference, including correction for unwanted phase contributions and temporal phase unwrapping. The correction of unwanted phase contributions specifically included the soil moisture dielectric effect. This effect was considered by predicting interferometric phase based on in situ measured soil moisture contents. The soil moisture dielectric effect was shown to be much smaller than shrinkage phase in our case study. A simple model was developed to estimate vertical shrinkage, using assumption on shrinkage behaviour (normal and isotropic shrinkage) and an approximation of water storage change from precipitation and evapotranspiration data. Using this model, temporal phase unwrapping results were corrected. The corrections for soil moisture dielectric phase and the correction of phase unwrapping both improved vertical shrinkage measurements from InSAR.

The results in this thesis make clear that vertical clay shrinkage can be estimated from InSAR. At the same time, these results show that clay shrinkage is a considerable phase contribution to interferometric phase and can therefore cause unwrapping and 
interpretation errors when not accounted for. To estimate vertical clay shrinkage from InSAR, a shrinkage model including assumptions of normal and isotropic shrinkage, proved useful in the phase unwrapping procedure in this case study. However, using the same assumptions to compute water storage change from these InSAR estimates, will in many cases produce inaccurate results. Therefore, in order to use InSAR for estimating soil water storage change in clay soils, the soil shrinkage characteristic, soil moisture dependency of the shrinkage geometry factor, and the effect of variable drying and wetting conditions, need to be considered. 



\section{Nomenclature}

Acronyms, symbols for parameters and variables, including their most common units, and operators:

\section{Acronyms}
ALOS
Advanced Land Observation Satellite (Japanese radar
CAT satellite)
COLE
Compact Active Transponder
COSMO-SkyMed
Coefficient Of Linear Extensibility
CS616
COnstellation of small Satellites for the Mediterranean basin Observation (Italian radar satellite constellation)

\section{EC-5 \\ DEM \\ DePSI \\ DInSAR \\ DORIS \\ DS \\ ENVISAT \\ ERS}
ESM
GPS
ILS
InSAR
KNMI
LIDAR
LOS
$\mathrm{MM}$
NSO
Water content reflectometer soil moisture sensor (Campbell Scientific)
Capacitance soil moisture sensor (Decagon)
Digital Elevation Model
Delft implementation of Persistent Scatterer Interferometry
Differential radar interferometry
Delft Object-oriented Radar Interferometric Software
Distributed Scatterer
ENVIronmental SATellite (European radar satellite)
European Remote Sensing Satellite (European radar satellites: ERS-1 and ERS-2)
Equivalent Single Master
Global Positioning System
Integer Least Squares
Interferometric SAR (also: Radar Interferometry)
Royal Netherlands Meteorological Institute (NL: Koninklijk
Nederlands Meteorologisch Instituut)
LIght Detection and RAnging
(Radar) Line Of Sight
Multi-Master
Netherlands Space Office 


$\begin{array}{ll}\text { PLE } & \text { Potential Linear Extensibility } \\ \text { PS } & \text { Persistent Scatterer } \\ \text { PSI } & \text { Persistent Scatterer Interferometry } \\ \text { RADARSAT } & \text { Canadian radar satellites (RADARSAT-1 and } \\ \text { Sentinel-1 } & \text { RADARSAT-2) } \\ \text { SAR } & \text { European radar satellites (Sentinel-1A and Sentinel-1B) } \\ \text { SBAS } & \text { Synthetic Aperture Radar } \\ \text { SHP } & \text { Small BAseline Subset } \\ \text { SLC } & \text { Statistically Homogeneous Pixels } \\ \text { SM } & \text { Single-Look Complex format } \\ \text { SNR } & \text { Single Master } \\ \text { SRTM } & \text { Signal-to-Noise Ratio } \\ \text { SSC } & \text { Shuttle Radar Topography Mission } \\ \text { SRTM } & \text { Soil Shrinkage Curve (also; shrinkage characteristic) } \\ \text { TDR } & \text { Shuttle Radar Topography Mission } \\ \text { TDX } & \text { Time Domain Reflectometry } \\ \text { TSX } & \text { TanDEM-X (German radar satellite) } \\ \text { WGS84 } & \text { TerraSAR-X (German radar satellite) } \\ \text { XRD } & \text { World Geodetic System 1984 } \\ & \text { X-Ray Diffraction }\end{array}$

\section{Symbols}

$\begin{array}{lll}\alpha & \text { Baseline orientation } & \circ \\ \gamma & \text { Coherence } & - \\ \varepsilon & \text { Fitting parameter to describe the soil shrinkage curve or } & - \\ \theta & \text { dielectric constant } & \\ & \text { Volumetric soil moisture content or look angle with } & \mathrm{cm}^{3} \mathrm{~cm}^{-3} \\ \vartheta & \text { respect to the reference surface } & \mathrm{or}^{\circ} \\ \lambda & \text { moisture ratio (volume of water over volume of solids) } & \mathrm{cm}^{3} \mathrm{~cm}^{-3} \\ \Phi & \text { Radar wavelength } & \mathrm{m} \\ \phi & \text { Normalized porosity } & - \\ \varphi & \text { Porosity (volume of pores over total soil volume) } & \mathrm{cm}^{3} \mathrm{~cm}^{-3} \\ \varphi_{\text {flat }} & \text { Interferometric phase } & \mathrm{rad} \\ \varphi_{\text {topo }} & \text { Topographic phase } & \mathrm{rad} \\ \varphi_{\text {defo }} & \text { Deformation phase } & \mathrm{rad} \\ \varphi_{\text {scat }} & \text { Scattering phase } & \mathrm{rad} \\ \varphi_{\text {atmo }} & \text { Atmospheric phase } & \mathrm{rad} \\ \varphi_{\text {orb }} & \text { Orbital phase } & \mathrm{rad} \\ \varphi_{\text {scat }} & \text { Scattering phase } & \mathrm{rad} \\ \varphi_{\text {noise }} & \text { Phase noise } & \mathrm{rad} \\ \psi & \text { (Fractional) phase in SAR image/SLC } & \mathrm{rad} \\ & & \mathrm{rad}\end{array}$




\begin{tabular}{|c|c|c|}
\hline$B$ & Baseline & $\mathrm{m}$ \\
\hline$B_{T}$ & Temporal baseline & yr \\
\hline$B_{\|}$ & Parallel baseline & $\mathrm{m}$ \\
\hline$B_{\perp}$ & Perpendicular baseline & $\mathrm{m}$ \\
\hline$D_{\text {LOS }}$ & Deformation in the radar line of sight (LOS) & $\mathrm{mm}$ \\
\hline$E T$ & Evapotranspiration & $\mathrm{mm}$ \\
\hline$H$ & Height above reference surface & $\mathrm{m}$ \\
\hline$H_{0}$ & Reference surface & $\mathrm{m}$ \\
\hline$M$ & master image (see also $m$ ) & - \\
\hline$N$ & Number of slave images & - \\
\hline$P$ & Complex phasor or Precipitation & - or mm \\
\hline$P_{0}$ & Point on reference surface $H_{0}$ & - \\
\hline$P_{H}$ & Point at height $H$ above reference surface & - \\
\hline$R^{2}, R^{2}$ & Coefficient of determination & - \\
\hline$S$ & $\begin{array}{l}\text { Slave image (see also } s \text { ) or water loss in the structural } \\
\text { shrinkage phase }\end{array}$ & - or $\mathrm{mm}$ \\
\hline$S_{\text {obs }}$ & Observed water loss in the structural shrinkage phase & $\mathrm{mm}$ \\
\hline$\Delta T$ & Satellite revisit time & $\mathrm{d}$ \\
\hline$U$ & Normalized water content & - \\
\hline$V$ & Clay aggregate or soil matrix volume & $\mathrm{cm}^{3}$ \\
\hline$\Delta V$ & Soil matrix volume change per unit area & $\mathrm{mm}$ \\
\hline$W$ & Soil water storage per unit area & $\mathrm{mm}$ \\
\hline$\Delta W$ & Water storage change per unit area & $\mathrm{mm}$ \\
\hline$a, a^{\prime}$ & Linear fitting parameter (slope) & - \\
\hline$b, b^{\prime}$ & Linear fitting parameter (offset) & - \\
\hline$d$ & $\begin{array}{l}\text { Soil moisture sensor depth or InSAR single difference } \\
\text { observation }\end{array}$ & $\mathrm{m}$ or $\mathrm{rad}$ \\
\hline$d d$ & InSAR double difference observation & rad \\
\hline$e$ & Void ratio (volume of pores over volume of solids) & $\mathrm{cm}^{3} \mathrm{~cm}^{-3}$ \\
\hline$f_{\text {cor }}$ & $\begin{array}{l}\text { Ratio between actual layer thickness } z(t) \text { and initial layer } \\
\text { thickness } z(0)\end{array}$ & - \\
\hline$f_{0}$ & Radar frequency & $\mathrm{Hz}$ \\
\hline$\Delta_{g r}$ & Average ground range pixel spacing & $\mathrm{m}$ \\
\hline$h$ & Pressure head & $\mathrm{m}$ \\
\hline$i$ & $\begin{array}{l}\text { Indication of resolution cell, soil moisture sensor or soil } \\
\text { layer under consideration }\end{array}$ & - \\
\hline$m$ & Master image & - \\
\hline$q$ & Fitting parameter to describe the soil shrinkage curve & - \\
\hline$r$ & Reference pixel & - \\
\hline$r_{\mathrm{s}}$ & Shrinkage geometry factor & - \\
\hline$s$ & Slave image & - \\
\hline$u$ & Gravimetric water content & $\operatorname{gg}^{-1}$ \\
\hline$z$ & Soil layer thickness (at saturation) & $\mathrm{m}$ \\
\hline$\Delta z$ & Soil layer thickness change (or vertical soil surface motion) & $\mathrm{m}$ \\
\hline
\end{tabular}




\section{Operators}

$(.)^{*} \quad$ Complex conjugate

$\exp ($.$) \quad Exponential function$ 


\section{1}

\section{Introduction}

\subsection{Research background and motivation}

The subsurface components of the hydrological cycle are soil water (between the soil surface and the groundwater level) and groundwater (below the groundwater level). Soil water and groundwater have intricate feedback relationships with local weather, regional and even global climate (Eagleson, 1978a; Randall et al., 2007; Van Heerwaarden et al., 2010), the terrestrial and aquatic ecosystems, and landscape evolution (Tucker \& Bras, 2000). The unsaturated zone of the soil plays a pivotal role: it determines the distribution of water over several flow routes (Milly, 1994; Western et al., 1999; Robinson et al., 2008). Incoming water, from precipitation or irrigation, can infiltrate the soil (replenishing the sub-surface water reservoir), evaporate from the soil surface (thereby cooling the soil surface), or run off by overland flow (feeding streams and lakes). Once infiltrated, the water can follow multiple flowroutes: i) reverse its route to the soil surface and leave the soil as evaporation, feeding back moisture to the atmosphere and cooling the surface; ii) percolate further to replenish ground water, to act as a long-term resource for potable water or ultimately feed surface water bodies, and; iii) be extracted by plant roots and leave the plants by transpiration to the atmosphere through the plants stomata, supporting primary production through photosynthesis. Together with changes in the amount of water stored in the soil, these fluxes constitute the soil water balance.

The availability of water to sustain each of these fluxes is largely determined by the storage capacity of the soil, which is determined by soil properties like porosity and hydraulic conductivity, and the soil water storage (Vereecken et al., 2008). The soil water storage is the actual filling state of the soil, or in other words, the amount 
of water that is actually present in the soil. When expressed relative to a given soil volume or mass, it is called the soil moisture content. Insight in soil properties and soil water storage change is crucial for water use planning, water management, and climate adaptive strategies (Eagleson, 1978b). This insight can help for example to determine how much of the expected precipitation can be stored in the soil and thereby aid flood forecasts. If changes in soil water storage can be quantified with short time intervals (e.g. days) along with above surface fluxes such as precipitation and evapotranspiration rate, water fluxes can be derived and deep groundwater recharge for long term water availability for potable water can be obtained. The porosity of mineral soils can be as high as $60 \%$, facilitating a huge water storage potential. Accurate and frequent estimation of soil water storage or soil water storage changes is therefore important for use in hydrological studies.

For hydrological studies and policy making, it is important to have measurements of soil water storage change on field to regional scales (i.e. $10^{-1}-10^{4} \mathrm{~km}^{2}$ ) and temporal resolution of days to weeks (Blöschl \& Sivapalan, 1995). Despite the recognized importance of quantifying soil water storage (Robinson et al., 2008; Vereecken et al., 2008), reliable measurement methods at these scales are scarce. Provided that the main fluxes that constitute the water balance can be estimated, the soil water storage change can be estimated, but in this way all neglected fluxes and estimation errors are accumulated in this term as well. The estimation of soil water storage change from contact-based soil moisture content sensors can provide accurate results, but are limited to small spatial scales. Upscaling of these point-measurements is hampered by heterogeneity of soil properties, land use, and precipitation and evaporation. Contactfree measurements of soil moisture content, such as remote sensing techniques and geophysical measurements like ground penetrating radar, can provide information on larger spatial scales. A major drawback of current remote sensing techniques is the limited penetration depth of the measurements (Wagner et al., 2007). Therefore soil water storage change estimations from remote sensing techniques depend largely on model applications, including assumptions and implementations of coupling between surface soil moisture (up to $5 \mathrm{~cm}$ depth) and deeper soil moisture, causing results to vary considerably (Kumar et al., 2009). Hydrogeophysical methods can offer field scale measurements of root zone soil moisture content, but temporal coverage is limited due to the campaign-based character of these measurements. Recently the use of cosmic-ray neutrons for soil moisture content measurements (Zreda et al., 2008) has been further developed, which can partly overcome these limitations as it enables estimates over horizontal scale of hectometres and at depths of decimetres and can be deployed at a site permanently (Zreda et al., 2012).

The lack of a reliable measurement technique, makes it desirable to explore new techniques and develop new methodologies based on these new techniques. These developments are needed, as hydrological models still produce highly uncertain representations of storage terms (Creutzfeldt et al., 2010). Therefore, this thesis explores the application of satellite based radar interferometry, or Interferometric Synthetic Aperture Radar (InSAR), to estimate soil water storage change. InSAR is a technique which is capable of measuring surface elevation changes at a scale of $10^{1}-10^{2} \mathrm{~m}^{2}$ with a potential differential precision in the order of millimetres (Gabriel et al., 1989). The 
relation between surface elevation changes and soil water storage change is known to exist for clay soils (Bronswijk, 1991b), as these soils swell and shrink upon wetting and drying. If the relations reported by Bronswijk (1991b) can be applied, measurements of surface elevation changes of a clay soil could be used to estimate soil water storage change in the entire unsaturated zone.

\subsection{Research objectives}

The main goal of this research is to develop the application of satellite based radar interferometry for estimation of soil water storage change on field scale to regional scale. As soil water storage change can never be a direct outcome of radar interferometric observations, using the technique for hydrological applications relies heavily on the physical relations between soil water storage change and clay swelling and shrinkage. Therefore, the main objective is refined in two research objectives:

1. Estimate soil water storage change from surface elevation changes in clay soils;

2. Measuring soil surface elevation changes from clay swelling and shrinkage on field to regional scale using satellite based radar interferometry.

Objective 1 deals with soil physics of clay soils in laboratory and field conditions, and requires quantification of the relation between clay volume change and water storage change. Objective 2 primarily deals with InSAR observations over clay soils, and investigates whether it is possible to unambiguously attribute InSAR phase observations to deformations resulting from clay dynamics.

\subsection{Concepts}

\subsubsection{Clay swell and shrinkage}

The property to swell upon wetting and shrink when drying out is specific to soils containing clay and some organic soils. The swelling and shrinkage ${ }^{1}$ behaviour of clay soils and organic soils differs, as organic matter interacts differently with moisture than clay minerals (Peng \& Horn, 2007; Nijp, 2015) and instable organic matter decomposes upon aeration, causing shrinkage of organic soils to be irreversible (Kennedy \& Price, 2005). Shrinkage in clays soils however is reversible, if soil structure is stable and shrinkage is not affected by ripening. The most visible effect of clay shrinkage are shrinkage cracks, the result of horizontal stretching of the soil (Fig. 1.1). Less visible to the naked eye are surface level elevation changes, the result of vertical shrinkage.

Clay shrinkage is often associated with vertisols and vertic-intergrade soils; soils with more than $30 \%$ clay, often consisting of the highly expansive montmorillonite mineral, exhibiting deep cracking when dried out. Shrinkage is, however, not limited to soils with vertic properties; also soils with lower clay content and consisting of other minerals are often subject to some degree of shrinkage. The distribution of soils with

\footnotetext{
${ }^{1}$ Different terminology is used throughout this thesis to indicate the process of volume change of clay soils under changing moisture conditions; clay swelling and shrinkage, just clay shrinkage, or clay dynamics. Reference to clay shrinkage or shrinkage potential is used in a universal sense to indicate the total process of both swelling and shrinkage.
} 


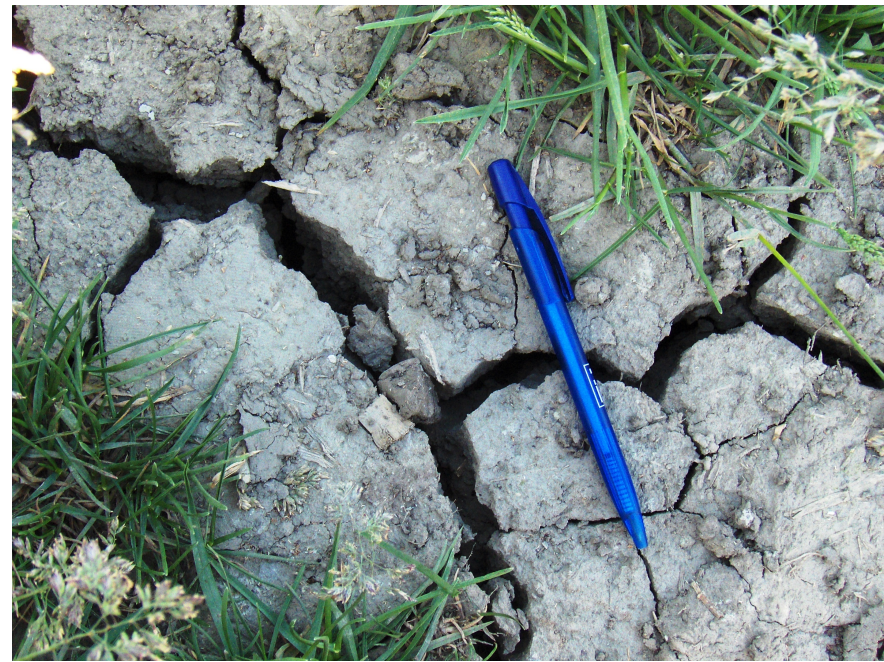

Fig. 1.1 Shrinkage cracks in a clay soil. Pen for scale.

minimum clay content of $25 \%$ (which is comparable to soils studied in this study), is mapped in Fig. 1.2, as an indication of swell-shrink potential without considering other governing factors like clay mineralogy, climate, soil chemistry, and water management. The abundance of potentially shrinking soils highlights the relevance of the current research.

Shrinking soils cause more direct problems in geotechnical engineering, as vertical and horizontal deformations cause economic damage to buildings and infrastructure (Nelson \& Miller, 1992; Corti et al., 2009, 2011; Jones \& Terrington, 2011). In terms of hydrology, studying shrinking soils is relevant, as shrinking soils provide challenges in soil water state and flux monitoring and modelling, due to non-consistency of soil properties (e.g. bulk density, porosity, and hydraulic conductivity). The hydraulic conductivity of a rigid clay soil for example is low, but temporary shrinkage cracks can provide fast transport routes for water, nutrients and pollutants to groundwater and surface water (Jarvis \& Leeds-Harrison, 1990; Wells et al., 2003). To characterise and parametrise the shrinkage process under laboratory and field conditions, substantial research efforts have been performed (e.g. Haines, 1923; Bronswijk, 1991a; Kirby et al., 2003; Boivin, 2007; Chertkov, 2012a).

Measurements of volume change in field soils are hard to obtain, but vertical shrinkage is easier to measure (Bronswijk, 1991b; Cabidoche \& Ozier-Lafontaine, 1995; Kirby et al., 2003; Neely et al., 2014). Assuming horizontal and vertical shrinkage to be equal (isotropic shrinkage), the total volume change can be derived from these measurements of vertical shrinkage. According to Bronswijk \& Evers-Vermeer (1990), clay soils in the Netherlands under Dutch climatic conditions shrink isotropically and experience a large range of normal shrinkage, defined as the phase in which water loss from the soil is fully compensated for by volume change. If this is the case, water storage change can be easily derived from vertical shrinkage measurements. 


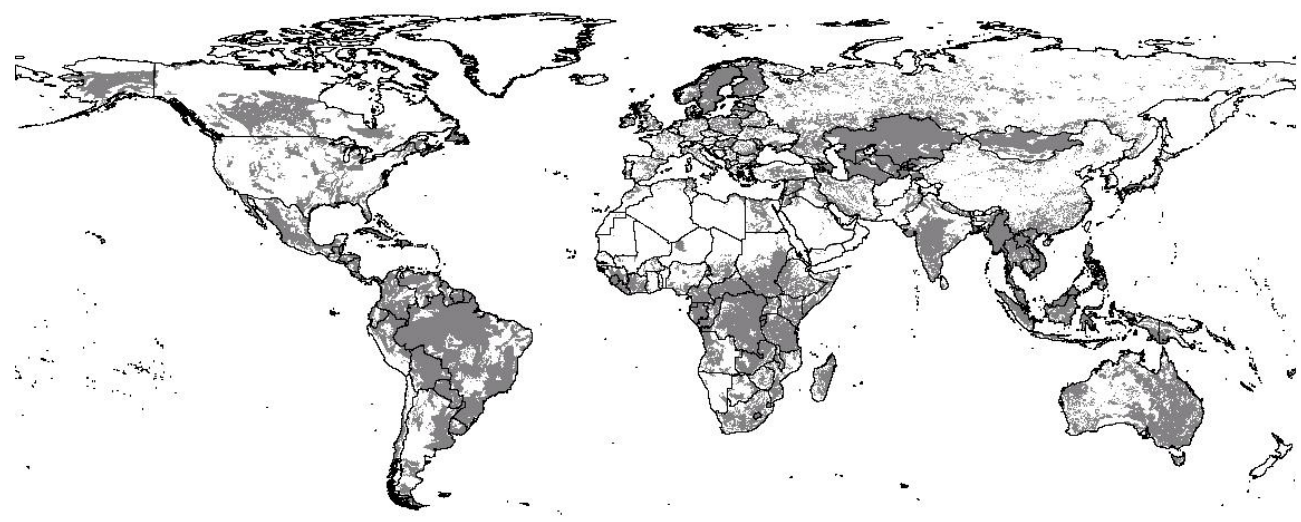

Fig. 1.2 Distribution of soils with minimum clay percentage of $25 \%$ in the upper $100 \mathrm{~cm}$. Data from the Harmonized World Soil Database, FAO/IIASA/ISRIC/ISSCAS/JRC (2012).

\subsubsection{Radar interferometry}

Satellite based radar interferometry, or radar interferometry in short, is a remote sensing technique capable of measuring small surface deformations. The technique uses an interferometric combination of two or more Synthetic Aperture Radar (SAR; hence the abbreviation InSAR) images, to measure relative surface elevation changes (Gabriel et al., 1989; Klees \& Massonnet, 1998). The surface elevation changes are derived from the phase information of the electromagnetic waves. Between transmission and reception of the electromagnetic waves at the radar antenna, many full wavelengths are covered, but upon reception only a portion of a full wavelength will remain. This portion, measured in degrees or radians, is called the phase of the wave. A change in phase between two SAR images acquired at different moments, can indicate a change in distance covered between transmission and reception and can therefore serve as a physical measurements of deformation (Gabriel et al., 1989; Bamler \& Hartl, 1998). SAR instruments can be ground-based or employed on a satellite platform or aircraft. In this thesis, data from the satellite platforms TerraSAR-X and TanDEM-X are used.

The result of interferometric processing of two SAR images is called an interferogram. Figure 1.3 shows an example of an interferogram, depicting ground displacement around the city of Bam, Iran, after the December 2003 earthquake. As in this example, the principal observation in interferograms is the two-dimensional relative phase, which is the $2 \pi$-modulus of the (unknown) absolute signal (Hanssen, 2001). The resulting $2 \pi$ phase-cycles are often referred to as fringes. Resolving the actual deformation from the fringes is called phase unwrapping and is one of the main challenges in radar interferometry (Goldstein et al., 1988; Gens, 2003).

Some main applications of satellite based radar interferometry are topographic mapping (e.g. Zebker \& Goldstein, 1986) and deformation mapping of volcanoes (e.g. Massonnet et al., 1995; Hooper et al., 2004; Spaans et al., 2015), earthquakes and landslides (e.g. Zebker et al., 1994; Rott \& Nagler, 2006; Diao et al., 2015), oil, gas, 


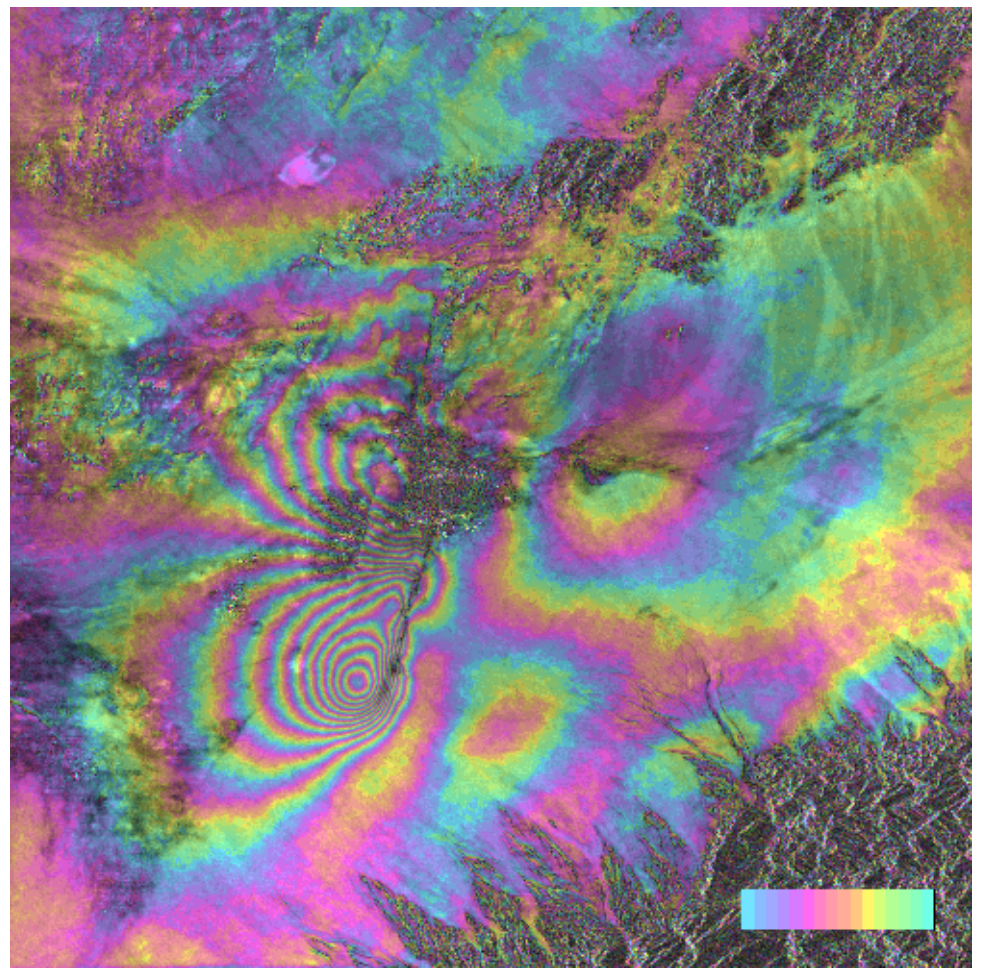

Fig. 1.3 Interferogram of the Bam (Iran) earthquake of 26 Dec. 2003 as measured by ENVISAT's ASAR sensor. One color cycle, or fringe, corresponds to $2.8 \mathrm{~cm}$ displacement in the line of sight to the sensor.

and geothermal reservoirs as a result of pumping (e.g. Massonnet et al., 1997; Ketelaar, 2009), man-made structures (e.g. Usai \& Klees, 1999; Chang \& Hanssen, 2015; Milillo et al., 2016), and groundwater reservoirs as a result of abstractions (e.g. Amelung et al., 1999; Reeves et al., 2014; Bonì et al., 2016a; Chen et al., 2016).

The relation between soil water storage change, clay shrinkage, and InSAR phase has so far not been quantified. In doing so, this thesis adds a potential application of InSAR to the list above. Although not aiming at estimation of soil water storage change, some InSAR studies have observed phase changes over agricultural fields related to unsaturated zone processes (Gens \& Van Genderen, 1996; Gabriel et al., 1989; Van der Kooij et al., 1995; Massonnet et al., 1997; Hanssen, 2001; Nolan \& Fatland, 2003; De Zan et al., 2014; Morishita \& Hanssen, 2015b; Zwieback et al., 2015).

\subsection{Thesis outline}

As the main technique considered to obtain large scale observations of soil water storage change in this thesis is satellite based radar interferometry, the reader is referred to Ch. 2 and references therein for theory of the principles of radar interferometry. Chapter 2 provides a brief introduction of the fundamentals of SAR scattering and 
radar interferometry for a broad audience, to provide necessary background information for further reading of this thesis. For a more detailed discussion of the technique and its applications, the reader is referred to one of the many reviews on radar interferometry (e.g. Gabriel et al., 1989; Gens \& Van Genderen, 1996; Bamler \& Hartl, 1998; Massonnet \& Feigl, 1998; Klees \& Massonnet, 1998; Hanssen, 2001).

Successful application of radar interferometry to measure vertical deformation as a result of swelling and shrinkage of clay, capitalizes greatly on the shrinkage potential of the soil. To determine shrinkage potential, a priori quantification of the relation between clay shrinkage and moisture change is proposed. In Ch. 3 soil swell-shrink potential of clay samples from the study area is assessed and the relation between soil volume and water content is quantified under laboratory conditions.

The scale difference of the relation between soil volume and moisture content in clay samples, clay aggregates in soil, and the bulks soil is addressed in Ch. 4, describing theory and field experiments in the Purmer area. Soil water storage estimations from surface elevation changes are compared to soil water storage changes measured with different types of soil moisture sensors. The chapter also characterises field shrinkage dependency on weather conditions and resulting drying regime. In this chapter the occurrence of normal shrinkage and the isotropic shrinkage assumption, simplifying the conversion of vertical shrinkage to soil water storage changes, are tested.

Chapter 5 describes a first application of satellite based radar interferometry over the study area and theoretical and practical considerations to obtain estimations of soil water storage change from clay shrinkage on field scale to regional scale using radar interferometry. Radar interferometric observations associated with application over rural terrain, including dependencies on soil type and land use, are characterised, helping in identifying potential areas of application. It is shown that phase signals can be linked to deformations resulting from frost heave in winter and from clay shrinkage in spring, based on double difference analysis.

Based on the results described in Ch. 5, the radar interferometric analysis over the study area is extended in Ch. 6 , in order to isolate the phase signal originating from clay shrinkage. Methodology and procedures to do so are described in this chapter, including an empirical model based on in situ observations to aid phase unwrapping. The resulting time series of interferometric phase is compared to in situ measurements of clay shrinkage and the significance of the clay shrinkage phase signal is compared to other phase contributions, to assess the potential of this methodology for hydrological applications and applications of radar interferometry.

In Ch. 7 the most important findings of this thesis are summarised, and it is discussed to what extent the research objectives are met. Implications of this thesis are described.

All abbreviations, variables, and parameters, including symbols and most commonly used units are listed in the Nomenclature. Due to conflicting symbol convention in radar interferometry, hydrology, and soil physics, some symbols represent multiple variables (e.g. $\theta$ for radar look angle and volumetric soil moisture content). In these cases, context will provide clarification about the variable at hand. The same counts for the term 'shrinkage phase', which is used for both the interferometric phase resulting from vertical clay shrinkage and for the indication of the relation between clay 
water content change and volume change as observed in the soil shrinkage characteristic (Haines, 1923). It is chosen to not deviate from terminology common in the scientific literature.

\subsection{Prospective audience}

The interdisciplinarity of the research in this thesis, could serve a number of audiences. Firstly, this thesis will be beneficial to hydrologists and applied geophysicists looking for new observation methods, steps forward in handling scaling issues, and integrating observations and models. Secondly, users of radar interferometry will benefit, as this thesis explains processes and mechanisms behind phase changes that can be observed in interferograms. Insights and methodology are provided to account for these mechanisms when these mechanisms themselves are not of prime interest in radar interferometric studies. Thirdly, all remote sensing scientists in general could use this thesis as an example of the much needed combination of field observations and remote sensing. In situ observations and field process understanding are key essentials in applying remote sensing techniques to Earth sciences, for validation, calibration and development of models and theories. 


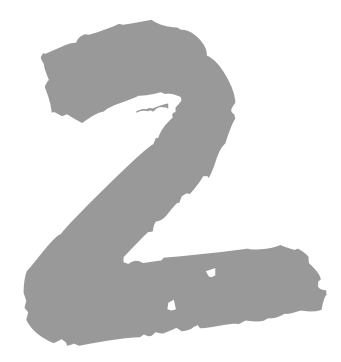

Radar Interferometry

The main technique considered to observe clay shrinkage in this research is Interferometric Synthetic Aperture Radar (InSAR), also known as radar interferometry. InSAR is a remote sensing technique capable of measuring subtle deformations of the Earth's surface, and therefore is a potential technique to observe vertical movement of the soil resulting from swell and shrinkage of clays. This chapter briefly introduces the fundamentals of SAR scattering and radar interferometry for a broad audience, partly based on Hanssen (2001) and Van Leijen (2014), to provides background information for further reading of this thesis. For a more detailed discussion the reader is referred to reviews of radar interferometry, e.g. Gabriel et al. (1989); Gens \& Van Genderen (1996); Bamler \& Hartl (1998); Massonnet \& Feigl (1998); Klees \& Massonnet (1998); Hanssen (2001).

\subsection{InSAR principles}

\subsubsection{Imaging radar observations}

The use of InSAR as a measurement technique for deformation monitoring (e.g. seismic movement) and topographic mapping is based on observations of phase difference between two radar images. These so-called Synthetic Aperture Radar (SAR) images are acquired by active microwave sensors on-board airborne or spaceborne platforms. Imagery is obtained by transmission of repeating pulses and reception of echos of each

This chapter is authored by Te Brake, B. 
pulse scattered back from the Earth's surface, resulting in what can be considered as a coarse image of returned echos from mapped objects. The (azimuth or along track) resolution of this image depends on the physical antenna length, and can be several kilometres large (Bamler \& Hartl, 1998). In a processing step called focussing the coarse resolution data is converted to high resolution SAR imagery, resulting in an increase in azimuth resolution of about three orders of magnitude (Hanssen, 2001). The focussed complex SAR data is often called Single-Look Complex (SLC) data.

$\mathrm{SAR}$ is a coherent imaging technique, meaning that both amplitude and phase of the electromagnetic signal are received and stored. The amplitude is determined by the backscatter intensity, whereas phase is mainly determined by the complex interaction of the electromagnetic signal with the Earth's surface. Amplitude and phase information per ground resolution cell is stored as complex phasor P (Hanssen, 2001). The retrieved complex phasor per ground resolution cell (or pixel) in a SAR image is formed by a summation of the reflections of all scattering objects present in the corresponding area on the Earth's surface (Curlander \& MacDonough, 1991). The retrieved amplitude and phase in the radar image therefore depend on the characteristics of all these objects. The amplitude is primarily determined by the dielectric properties, orientation and roughness of the scatterers, and sensor properties such as wavelength and incidence angle (Hallikainen et al., 1985; Curlander \& MacDonough, 1991; Ulaby et al., 1996; Richards, 2009). Dielectric properties are affected by moisture content and material properties, with a higher dielectric constant causing a higher backscatter intensity (Ulaby et al., 1996). The way the incoming radar signal is reflected to the antenna (the scattering mechanism) can largely affect measured backscatter intensity, phase and the relative contribution of each scatterer to the measurements per resolution cell.

\subsubsection{Radar scattering mechanisms}

Considering the response of one resolution cell in a SAR image, there are two main cases of scattering, yielding highly different responses; 1) distributed scattering, and 2) point scattering (Bamler \& Hartl, 1998).

Distributed scattering (or Gaussian scattering) yields contributions from many individual scatterers in the resolution cell. The contributions of the scatterers to the resolution cells response in the SAR image cannot be discriminated and there is no dominant scatterer (Fig. 2.1A and B). Distributed scattering can be subdivided in two underlying mechanisms; surface scattering and volume scattering (Bamler \& Hartl, 1998; Richards, 2009). Surface scattering occurs when the incoming radiation encounters a substantial discontinuity in dielectric constant $\varepsilon$, e.g. from air to soil. For the latter, the backscatter intensity of a wet soil is higher than that of a dry soil, owing to a higher dielectric constant of the soil and thus a larger discontinuity between air and the soil ${ }^{1}$. Furthermore, the backscatter intensity of a rough surface is higher than that of a smooth surface as reflection from a smooth surface is highly specular (i.e. it acts as a mirror and reflects a large

${ }^{1}$ The dielectric constant of air is roughly 1 , while the dielectric constant of soils can vary from 3 to 40, depending on soil texture and moisture state (Topp et al., 1980). 
portion of the incoming energy away from the radar antenna). Volume scattering occurs when incoming radiation penetrates into a medium and experiences an uncountable number of reflections on multiple dielectric discontinuities, such as the components of tree or crop canopy, or inhomogeneities in soils. The penetration into the medium and the number of reflections determine the energy loss of the radiation, and thereby the backscatter intensity. Often, distributed scattering is a combination of surface and volume scattering, as the microwave signal might not fully scatter on a medium's surface (e.g. soil), but also penetrate into it (Curlander \& MacDonough, 1991). The latter effect is particularly large for dry and loose soils and for long wavelength signals (e.g. L-band SAR). The dependency of backscatter intensity on wetness forms the basis for SAR soil moisture retrieval techniques (Dubois et al., 1995; Ulaby et al., 1996), but the simultaneous dependency on soil roughness, crop cover and other soil properties hampers operational use (Wagner et al., 2007; Mattia et al., 2009; Lievens et al., 2011).

Point scattering In the case of point scattering, the resolution cell's response is determined by 'hard target' scattering. Hard target scattering can lead to very dominant scattering from one single scatterer in the resolution cell, causing this dominant scatterer (point scatterer) to be responsible for the retrieved information of this resolution cell. The surrounding scatterers in the resolution cell are only adding noise, or clutter to the point scatterers response (Fig. 2.1C and D). The strong response from hard target scattering can be caused by different types of scattering interactions, like facet scattering on flat reflectors or multiple bounce scattering on natural or man-made dihedral or trihedral reflectors oriented towards the incoming radar beam.

The phase due to scattering characteristics of a resolution cell (scattering phase is unpredictable, but it is a deterministic quantity: if interaction between the electromagnetic wave and a scatterer or a group of scatterers is the same, the phase of a second observation will be exactly the same as the phase of the first observation (Zebker \& Villasenor, 1992; Hanssen, 2001). The imaging in this case is called coherent. The degree of coherence is a measure for the correlation between two observations. Coherent imaging is an important prerequisite for successful information retrieval from an interferometric combination of SAR images. Note that although the targets and scattering mechanisms can remain the same over time, the physical location of targets can change (e.g. subsidence of a house or seismic movement) resulting in phase change.

Sensor characteristics influence the scattering mechanisms that occurs, the scatterers response, and the degree of coherence loss of the signals in time. For instance, signal wavelength and incidence angle influence the amount of penetration and volume scattering (Curlander \& MacDonough, 1991), and revisit time $\Delta T$ influences the temporal decorrelation of two acquisitions (Zebker \& Villasenor, 1992). The revisit time is the time it takes for a satellite to re-pass over an area in the same orbit. A list of prominent space-borne SAR systems and their characteristics can be found in Tab. 2.1. 


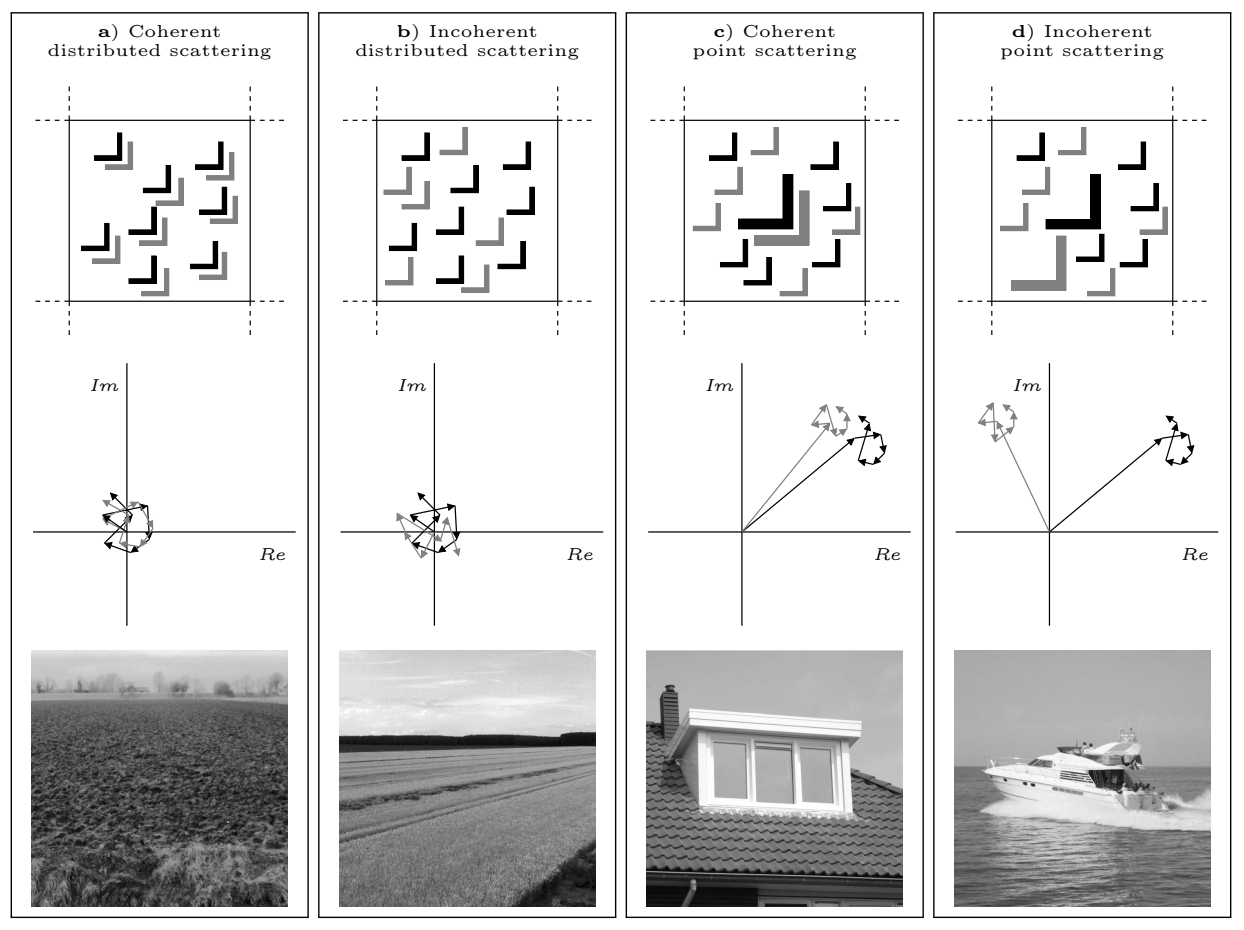

Fig. 2.1 Distributed scattering and points scattering in case of coherence and incoherence. Top; scattering objects within a resolution cell at two acquisitions (indicated by black and gray reflecting objects). A large object corresponds to a strong reflection, whereas the small objects represent weak reflections. Middle; phasors for the two acquisitions. Bottom; examples of scattering objects. The examples for distributed scattering are obtained in the current research; bare agricultural field in wintertime (coherent) and a cropped field during the growing season (see Ch. 5). (Figure adapted from Van Leijen (2014).)

\subsubsection{Single-Look Complex phase}

The Single-Look Complex (SLC) format is the starting point of the discussion here and the analysis in this thesis. The observed SLC phase for a single pixel can be written as

$$
\psi=-2 \pi a+\psi_{\text {range }}+\psi_{\text {scat }}+\psi_{\text {atmo }}+\psi_{\text {noise }},
$$

where $a$ is the integer phase ambiguity number, i.e. the number of full phase cycles, $\psi_{\text {range }}$ is the phase proportional to the distance between sensor and target (range dependent phase), $\psi_{\text {scat }}$ is the scattering phase, $\psi_{\text {atmo }}$ is the phase caused by atmospheric signal delay, and $\psi_{\text {noise }}$ is the phase noise. The scattering phase represents the clutter from many scatterers in the resolution cell whereas the phase noise represents the noise introduced by the imaging system (e.g. thermal noise) (Van Leijen, 2014).

Neglecting variable sources like atmospheric composition and noise for now, the above means that we can regard the SLC phase observation as the sum of two contributions: range dependent phase and scattering phase. For distributed scattering, 
Table 2.1 List of satellite SAR missions suitable for interferometry, including some main characteristics; $\Delta T$ is the revisit time, $\lambda$ the radar wavelength, $f_{0}$ the carrier frequency, $\theta$ the look angle and $\Delta_{g r}$ the average ground range pixel spacing (stripmap/standard mode).

\begin{tabular}{lccccccc}
\hline mission & $\begin{array}{c}\text { altitude } \\
{[\mathrm{km}]}\end{array}$ & years in orbit & $\begin{array}{c}\Delta T \\
{[\mathrm{~d}]}\end{array}$ & $\begin{array}{c}\lambda \\
{[\mathrm{cm}]}\end{array}$ & $\begin{array}{c}f_{0} \text { (band) } \\
{[\mathrm{GHz}]}\end{array}$ & $\begin{array}{c}\theta \\
{[\mathrm{deg}]}\end{array}$ & $\begin{array}{c}\Delta_{g r} \\
{[\mathrm{~m}]}\end{array}$ \\
\hline Seasat & 800 & 1978 & 3 & 23.5 & $1.275(\mathrm{~L})$ & 23 & 25.0 \\
ERS-1 & 790 & $1991-2000$ & $35^{a}$ & 5.7 & $5.300(\mathrm{C})$ & 23 & 20.0 \\
ERS-2 & 790 & $1995-2011$ & 35 & 5.7 & $5.300(\mathrm{C})$ & 23 & 20.0 \\
RADARSAT & 792 & $1995-2013$ & 24 & 5.6 & $5.300(\mathrm{C})$ & $20-49$ & 30.0 \\
Envisat & 800 & $2001-2012$ & 35 & 5.7 & $5.300(\mathrm{C})$ & 23 & 20.1 \\
ALOS & 690 & $2006-2011$ & 46 & 23.6 & $1.270(\mathrm{~L})$ & $8-60$ & 7.5 \\
RADARSAT-2 & 798 & 2007 -now & 24 & 5.5 & $5.405(\mathrm{C})$ & $20-49$ & 21.1 \\
TerraSAR-X & 514 & 2007 -now & 11 & 3.1 & $9.650(\mathrm{X})$ & $20-45$ & 2.1 \\
COSMO-SkyMed $^{c}$ & 620 & 2007 -now & 8 & 3.1 & $9.600(\mathrm{X})$ & $20-60$ & 2.2 \\
ALOS-2 $^{b}$ & 628 & 2014 -now & 14 & 23.6 & $1.270(\mathrm{~L})$ & $8-70$ & 6.0 \\
Sentinel-1A $^{2}$ and $1 \mathrm{~B}$ & 693 & $2014^{d}$-now & $6^{e}$ & 5.7 & $5.405(\mathrm{C})$ & $29-46$ & 3.7 \\
\hline
\end{tabular}

${ }^{a}$ For majority of the mission's lifetime; orbit configuration adapted.

${ }^{b}$ TerraSAR-X and its 'twin brother' TanDEM-X are identical and flying in controlled formation ideal for topographic mapping. The satellites are individually or combined suitable for interferometry. ${ }^{c}$ Constellation of 4 satellites.

${ }^{d}$ Sentinel-1B launched in 2016.

${ }^{e}$ When combining acquisitions of both satellites. Individual satellites have a 12 day revisit time.

with a sufficiently high number of scatterers per resolution cell so that the SLC pixel value is a complex circular Gaussian random variable, the probability density function of phase is uniform due to the summation over many scatterers of random phase (Bamler \& Hartl, 1998). As such, no relevant information can be retrieved from the phase of a single SLC. However, the phase of the complex product of two circular Gaussian signals is not necessarily uniformly distributed as long as the observations have some degree of coherence (see for examples and further discussion Ch. 5). Still neglecting atmospheric phase and phase noise and assuming fully coherent imaging, the total phase difference only depends on the difference in range, as the scattering phase contribution cancels. By applying interferometric processing, the phase differences of sufficiently coherent scattering can be calculated and serve as a direct measurement of range distance between sensor and target, provided that other phase contributions are small with respect to the range dependent phase.

\subsubsection{Interferometric phase}

The interferometric combination of two SLC images, also called master and slave, can be performed after exact alignment or coregistration of the images. An interferogram is created by the pixel-wise complex multiplication of the SLC images. Expressed as complex phasors per pixel $P^{\mathrm{m}}$ and $P^{\mathrm{s}}$ this yields

$$
P^{\mathrm{ms}}=P^{\mathrm{m}} P^{\mathrm{s} *}=A^{\mathrm{m}} A^{\mathrm{s}} \exp \left(i\left(\psi^{\mathrm{m}}-\psi^{\mathrm{s}}\right)\right),
$$

where $(.)^{*}$ denotes the complex conjugate and $m$ and $s$ indicate the master and slave SLC. The interferometric phase $\varphi$ for a single pixel is the difference in phase between master and slave: 


$$
\varphi=\psi^{\mathrm{m}}-\psi^{\mathrm{s}}
$$

Equation 2.3 can also be written in terms of phase contributors:

$$
\varphi=-2 \pi a+\varphi_{\text {flat }}+\varphi_{\text {topo }}+\varphi_{\text {defo }}+\varphi_{\text {atmo }}+\varphi_{\text {orb }}+\varphi_{\text {scat }}+\varphi_{\text {noise }} .
$$

Equation 2.4 is similar to Eq. 2.1, but the range dependent phase is subdivided in contributions from a flat Earth component $\varphi_{\text {flat }}$, topographic component $\varphi_{\text {topo }}$, and deformation component $\varphi_{\text {defo }}$. Additionally a contribution from errors in orbit parameters from master and slave acquisitions is included.

From Eq. 2.4 we can see that there are many contributions to the total observed interferometric phase. The most common variables of interest in InSAR studies are phase due to displacement, $\varphi_{\text {defo }}$ and phase due to topography $\varphi_{\text {topo }}$. To get to a system of variables that can be solved for the variable of interest, physical and geometric relationships between master and slave acquisition and interferometric phase contributors can be applied.

The contribution from flat Earth phase, topographic phase, and deformation phase are discussed below, based on a repeat-pass configuration in Fig. 2.2. Additionally, atmospheric phase and phase noise are discussed.

\section{Flat Earth phase}

Flat Earth phase or reference phase is caused by the non-constant distance between a reference body, e.g. an ellipsoid such as WGS84, and the satellite orbits. To compute the expected phase contribution, the satellite orbits during master and slave image acquisition and the reference body need to be modelled. Apparent satellite positions at master and slave acquisitions with respect to the reference surface can then be geometrically computed for every resolution cell. Using the assumption of parallel travel paths between target and satellite at master and slave acquisition (the far-field or parallel-ray approximation (Zebker \& Goldstein, 1986)) this leads to:

$$
\varphi_{\text {flat }}=\frac{4 \pi}{\lambda} B \sin (\theta-\alpha),
$$

where $\lambda$ is the radar wavelength, $B$ is the baseline, defined as the distance between master and slave antenna position, $\theta$ is the look angle with respect to the reference surface and $\alpha$ is the baseline orientation (Fig. 2.2). The look angle $\theta$ differs slightly from the (local) incidence angle $\theta_{\text {inc }}$, due to the curvature of the Earth. The difference in path length between master and slave acquisition (second term in Eq. 2.5) is directly proportional to the phase and is called the parallel baseline $B_{\|}$. Uncorrected flat Earth phase appears in interferograms as strong trends (Fig. 2.3), which can also be estimated and corrected without orbit information. A similar procedure can be applied to eliminate phase cause by inaccurate orbital information $\varphi_{\text {orb }}$ (Fig. 2.4).

\section{Topographic phase}

Like flat Earth phase, the phase caused by topography over the imaged scene also depends on the relative positions of the satellite's antenna at master and slave acquisition time. Topographic phase describes the phase contributions from height differ- 


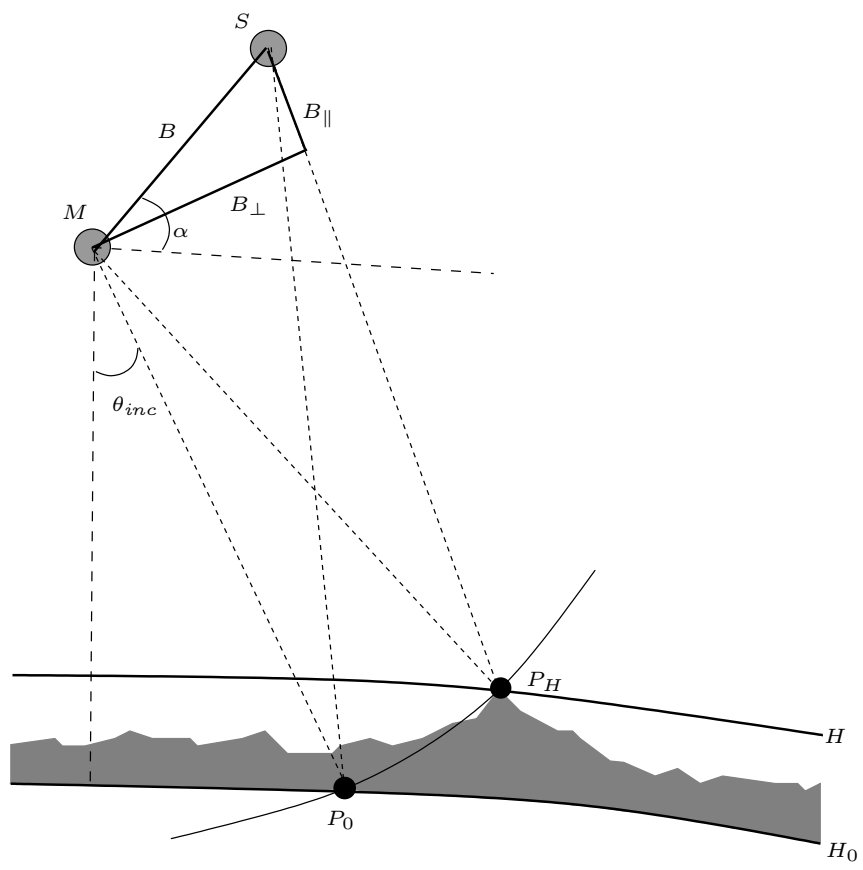

Fig. 2.2 Baseline configuration in case of repeat-pass radar interferometry. The orbit trajectories are perpendicular to the paper. The baseline $B$ is defined as the distance between the master $(M)$ and slave $(S)$ antenna position. The parallel baseline $B_{\|}$determines the flat Earth phase; the phase contribution due to a reference surface $H_{0}$ through point $P_{0}$. The topographic height $H$ of a point $P_{H}$ above the reference surface determines the topographic phase, which is a function of the perpendicular baseline $B_{\perp}$. The parallel and perpendicular baseline are both dependent on the

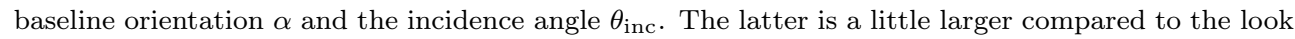
angle $\theta$ due to the curvature of the Earth. (Figure adapted from Van Leijen (2014).)

ences above the reference surface. Again using the far-field approximation, we can derive a geometrical relation between height above the reference surface and phase:

$$
\varphi_{\text {topo }}=-\frac{4 \pi}{\lambda} \frac{B \cos (\theta-\alpha)}{R \sin \theta H},
$$

where $R$ is the range distance from the master antenna and $H$ is the height above the reference surface. The term $B \cos (\theta-\alpha)$ is called the perpendicular baseline $B_{\perp}$ (Fig. 2.2). The sensitivity of phase change to topography increases with increasing perpendicular baseline. Illustrating the sensitivity of the system for TerraSAR-X, with orbital altitude $514 \mathrm{~km}, \lambda=0.031 \mathrm{~m}, B_{\perp}=500 \mathrm{~m}$ and $\theta_{\text {inc }}=30^{\circ}$; the phase of 1 meter height difference is $0.68 \mathrm{rad}$, meaning one full phase cycles corresponds to $9.2 \mathrm{~m}$ topographic height.

To study topography, interferometric pairs with relatively large baselines are preferred, provided that the temporal baseline, the time between master and slave acquisition, is short. To study deformation, the topographic phase caused by non-zero baselines has to be removed. This is know as differential interferometry or DInSAR (Gabriel et al., 1989). Topographic phase can be removed by use of external 


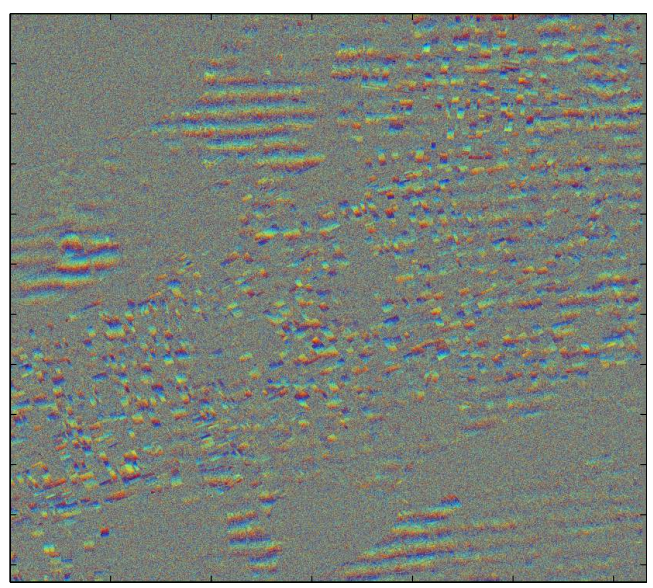

Fig. 2.3 Example of an interferogram not corrected for flat Earth phase and orbit errors, showing a strong phase trend.

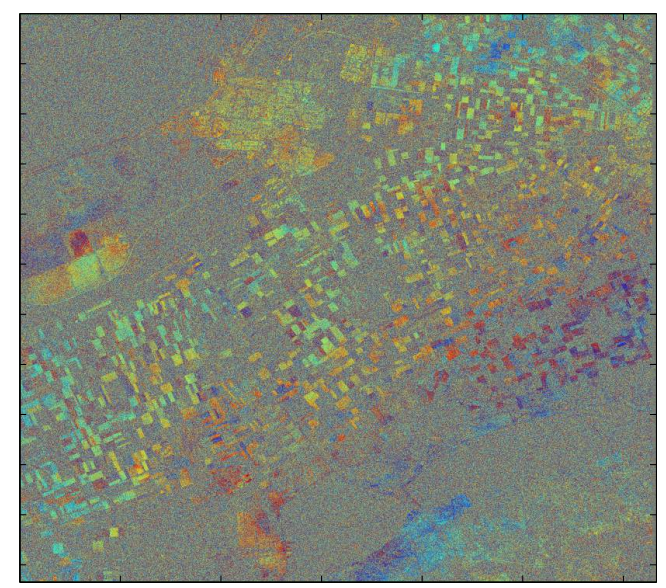

Fig. 2.4 Interferogram from Fig. 2.3 with phase trend due to flat Earth phase and orbit errors removed. (Image from RADARSAT-2 over Flevoland, the Netherlands.)

topographic information (two-pass method), or topography inferred from a second interferometric pair in case of absence of deformation (three-pass or four-pass method; Gabriel et al., 1989; Zebker et al., 1994).

\section{Deformation phase}

Supposed that flat Earth phase and topographic phase are not present or fully corrected for in the processing, the only range dependent phase comes from displacement of the scatterers. This forms the basis for accurate deformation measurements. Considering the relative positions of master and slave antenna, deformation causes a direct change in travel path lengths and therefore a phase change:

$$
\varphi_{\text {defo }}=-\frac{4 \pi}{\lambda} \mathrm{D}_{\mathrm{LOS}},
$$

where $\mathrm{D}_{\mathrm{LOS}}$ is the deformation in the radar line of sight (LOS). Note that $\varphi_{\text {defo }}$ only depends on the degree of displacement and the sensors wavelength $\lambda$. For TerraSAR-X with $\lambda=0.031 \mathrm{~m}$, this means that one full phase cycle represents a LOS displacement of $15.5 \mathrm{~mm}$. The sensitivity of phase to deformation is therefore much higher than the sensitivity to topography.

The line of sight deformation can be caused by vertical or horizontal displacement, which can not be resolved based on one interferogram. Combination of interferograms from tracks with different viewing positions (i.e. ascending and descending orbits) can be used to resolve the direction of displacement. Often an assumption of displacement direction is made based on the expected deformation process (Van Leijen, 2014).

Deformation measurements are based on a relative line of sight movement of scatterers with respect to a reference location in an interferogram. A difference in penetration depth of the microwave signal between master and slave acquisition will cause a sim- 
ilar effect, provided that scattering remains coherent, and cannot be distinguished from deformation. Penetration of the signal will however only occur over natural terrain, like canopy cover or soil, and will in most cases lead to substantial coherence loss (or decorrelation) due to volume scattering and the emergence of new scatterers (as discussed by De Zan et al., 2014).

\section{Atmospheric phase}

A difference in atmospheric composition between master and slave acquisition can cause a phase shift and thereby a contribution to the interferometric phase. The atmospheric phase contribution $\varphi_{\text {atmo }}$ is caused by differential delay of the signal resulting from variations in pressure, temperature (e.g. Hanssen, 2001) and water vapor (e.g. Goldstein, 1995; Hanssen \& Klees, 1999). Differential atmospheric delay mainly stems from the ionosphere $(50-2500 \mathrm{~km}$ height $)$ and the troposphere $(0-25 \mathrm{~km}$ height), and can be caused by turbulent mixing or vertical stratification (e.g. over mountainous terrain; Hanssen \& Klees, 1999)).

The resulting atmospheric phase can be present in an interferogram as either a long (>50 km) or short wavelength $(<10 \mathrm{~km})$ signal (Caro Cuenca, 2012). Long wavelength atmospheric signals have a similar effect as orbital errors and can often be simultaneously removed, without disentangling the two contributions. Short wavelength signals are much more variable and often very similar to deformation patterns due to a high spatial correlation. In this case, atmospheric phase corrections are difficult to apply without the risk of enhancing/diminishing the apparent deformation signal.

Using multiple interferometric combinations (in multi-temporal processing techniques), the atmospheric phase contribution in some of the interferograms can be estimated and corrected for. Also the use of high-resolution weather models has been proposed to eliminate the atmospheric phase contribution (Liu et al., 2011; Gong et al., 2015a,b). Recently a growing interest has emerged in using the atmospheric phase contribution as the main parameter of interest, e.g. for (hydro-)meteorological studies (Alshawaf et al., 2015).

\section{Scattering phase and noise}

Scattering phase $\varphi_{\text {scat }}$ is essentially an additional noise factor, originating from differences in scattering between master and slave acquisition, and therefore contributes to decorrelation of the interferogram. In extreme cases of decorrelation, the interferometric combination of two SAR images is insufficiently coherent and no phase information can be deduced from the interferogram. Over distributed scatterers the most prominent form of decorrelation is temporal decorrelation, caused by physical changes of the imaged surface in between image acquisitions. Several other sources of decorrelation are geometric decorrelation, caused by differences in master and slave incidence angles (Gatelli et al., 1994), doppler centroid decorrelation caused by difference in master and slave antenna orientation, thermal or system decorrelation, caused by instrumental noise, and processing decorrelation caused by errors in the processing results (e.g. coregistration or interpolation errors). These sources and their relation with imaging parameters and geometry are extensively discussed by Zebker \& Vil- 
lasenor (1992), Hanssen (2001), and Van Leijen (2014). All sources of decorrelation are multiplicative, resulting in a total coherence (Zebker \& Villasenor, 1992). Coherence and decorrelation in the context of agricultural terrain and application to vadose zone processes will be further discussed and illustrated in Ch. 5. Minimizing scattering phase and noise in interferograms can be achieved by careful selection of interferometric combinations (e.g. small baselines pairs), sensor (and thereby wavelength and revisit time), or by applying additional processing steps (e.g. oversampling, spectral filtering, spatial filtering or multi-looking).

\subsection{Interferometric processing}

\subsubsection{Conventional InSAR}

To extract the relevant phase information from the combination of SAR data, interferometric processing is needed. The most important processing steps are listed below. A detailed description of processing steps is outside the scope of this thesis. For this the reader is referred to Hanssen (2001).

As described in Sec. 2.1.1, focussing of the raw radar data is applied to improve spatial resolution and obtain SAR images in the SLC format. Often, as in this study, the SLC data is the starting point for interferometric processing. Additionally precise orbital information is needed to be able to apply the geometric relations described in 2.1.4. The main steps in preparation of the SLCs for interferogram computation are coregistration of SLC's and resampling of the slave image so that it is an exact subpixel overlay of the master image. Filtering to reduce phase noise in the interferogram can be carried out on the master and slave images prior to interferogram computation or on the interferogram itself after its computation. Interferogram computation is performed by the pixel-wise complex multiplication of the SLC images, leading to a complex phase image. The interferogram at this stage still has to be corrected for unwanted phase contributions, for example flat Earth phase and topographic phase for deformation studies. To do so, the relations described in Sec. 2.1.4 are used. The next crucial step is to unwrap the phase data, also known as ambiguity resolution. This is necessary as interferograms only contain the relative phase, wrapped to the $[-\pi,+\pi)$ interval. Phase unwrapping is the procedure to estimate the absolute phase value from the integer phase ambiguity number ( $a$ in Eq. 2.4). To do so, assumptions on the phase gradient in the interferogram are needed (Hanssen, 2001). In single interferograms phase unwrapping is often performed by integrating 2-dimensional wrapped phase gradients, assuming that the absolute phase gradient is smooth and less than half a phase cycle (i.e. less than $\pi$ ) between adjacent pixels (e.g. Goldstein et al., 1988). In the final step of the processing the unwrapped phases are converted to topographic height (again based on geometric relations between master and phase acquisition), and conversion from radar coordinates to a geodetic reference system (geocoding).

\subsubsection{Multi-interferogram techniques}

Due to the superposition of several phase contributions per pixel in an interferogram, elimination or suppression of unwanted signals can not always be achieved by com- 
bining two SAR images. Often however, this can be done by constructing a network of multiple images, to allow computation or estimation of the phase relations within the network. Relative straight forward examples of this approach are the three- and four-pass method to eliminate topography for deformations studies (Gabriel et al., 1989; Zebker et al., 1994). More advanced methods can be applied for mitigation of other contributions (e.g. atmospheric phase) and to construct a time line of phase change over a series of SAR images.

The need for high quality observations for retrieving time series of displacement, has led to the development of Persistent Scatterer (PS) techniques (e.g. Ferretti et al., 2000, 2001; Hooper et al., 2004). These techniques rely on a selection of point-like radar targets that remain phase-coherent over long periods of time and over different imaging geometries. In practice, these are mostly man-made structures and bare surfaces such as rock outcrops exhibiting point scattering (Sec. 2.1.2), resulting in a sparse grid of measurement points. All observations are related to each other through a common master image in a network of observations. This network can then be used for stochastic analysis and estimation of the atmospheric component, efficient phase unwrapping, and construction of time series of deformations.

PS-techniques are not suitable to estimate deformation over natural environments, because here phase statistics are generally not preserved over long periods. Still, some areas over natural terrain might still exhibit good coherence levels over shorter time spans or for certain imaging geometries. This is the fundamental idea of smallbaseline techniques (e.g. Berardino et al., 2002). To obtain high-quality observations, only combinations of images with a sufficiently small perpendicular baseline are used, which are connected through common images. This is a difference with PS-techniques, which use a common master for all interferograms, and means that DS-techniques can potentially use more interferograms to extract information from than the total number of SAR images available.

Some studies have combined PS- and DS-techniques to increase spatial density of measurement points and maintain high quality observations over both urbanized and natural terrain (Hooper, 2008; Goel \& Adam, 2014; Morishita \& Hanssen, 2015b). Also, time series processing techniques have been developed to integrate processing of persistent and distributed scatterers in a unified processing scheme. These techniques first extract the maximum possible information from distributed scatterers from a stack of interferograms, and then jointly process PS and DS pixels together in an hybrid manner (Monti Guarnieri \& Tebaldini, 2008; Ferretti et al., 2011; SamieiEsfahany et al., 2016). To reduce the impact of the effect of decorrelation on DS, the techniques use multilooked wrapped phases from multi-master interferograms, and subsequently estimate an equivalent single master (ESM) phase time series from this multi-master stack of interferograms. In other words; first, phase differences over DS in all possible combinations of acquisitions are multilooked, and second, all these phase differences are combined in an optimal way to estimate the phase differences with respect to one acquisition, called the (super) master. The ESM-phase estimation is called phase linking (Monti Guarnieri \& Tebaldini, 2008) or phase triangulation (Ferretti et al., 2011). ESM-phase estimation is applied in Ch. 6. 



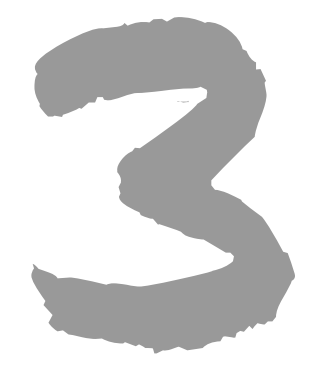

\section{Shrinkage potential, geometry, and moisture content relations of clay aggregates from a Dutch polder}

\subsection{Introduction}

The presence of clay minerals in a soil may cause the soil to exhibit shrinkage when drying and swelling when (re-)wetted. The magnitude of shrinkage and swelling depends on the combined effect of soil properties and environmental factors. Quantification of clay swelling and shrinkage with water content change is needed for soil physical model description and process understanding. In this thesis, measurements of clay shrinkage potential and relations between volume change and moisture content change are needed to assess the potential of radar interferometry to measure swelling and shrinkage, and subsequently derive soil water storage change. The potential of a soil, soil layer, or soil aggregates to shrink is called the shrinkage potential, and can be interpreted as the maximum shrinkage that occurs under ideal drying conditions. These conditions do generally not occur in a field situation, and therefore shrinkage potential and the relation with soil water content are determined by laboratory measurements.

The property to swell and shrink with water content changes in soils is attributed to the presence of clay minerals, such as kaolinite, illite and montmorillonite. Clay minerals generally consist of platelets. As a result of electrical surface charges of the

This chapter is a modified version of: Te Brake, B. and Van der Ploeg, M.J.: Shrinkage potential, geometry, and moisture content relations of clay aggregates from a Dutch polder. In preparation. 
platelets, water molecules are contained in the spaces between platelets. The amount of water contained within these inter-platelet spaces depends on the soil water content around the (packet of) platelets: water is released from the inter-platelet spaces when the soil dries out, resulting in smaller inter-platelet spacing and shrinkage of soil aggregates. The opposite happens when the soil is (re-)wetted; inter-platelet spacing increases due to absorption of water, and the aggregates regain their original volume (swelling) (Nelson \& Miller, 1992).

Both soil properties and environmental factors affect the swelling and shrinkage potential and behaviour of clay soils. One of the soil properties is type of clay mineral. Generally, kaolinite does not exhibit shrinkage, illite can exhibit limited shrinkage, and montmorillonite is can exhibit strong shrinkage (Nelson \& Miller, 1992, and citations therein). Some other soil properties of influence are clay content, soil structure, and soil chemistry (Parker et al., 1977). The main environmental factor is moisture variation, influenced by climate, groundwater level, vegetation, and drainage or irrigation.

The swelling and shrinkage potential of a soil can be assessed by several laboratory tests on soil aggregates (e.g. Parker et al., 1977), ranging from determination of clay content and mineralogy to determination of linear extensibility and full quantification of the aggregate volume - moisture content relation, through the shrinkage characteristic curve. Assuming only external shrinkage of aggregates, several stages, called the shrinkage phases, can be distinguished upon drying (Haines, 1923; Bronswijk \& Evers-Vermeer, 1990; Chertkov, 2007a):

1. Structural shrinkage: no or limited volume change upon first drying and dewatering of large pores;

2. Basic shrinkage: volume decrease with water loss is linear and constant (this phase is often referred to as normal shrinkage if volume decrease equals water loss);

3. Residual shrinkage: water loss exceeds volume change as limited water is released from the clay minerals, but mainly from isolated pockets or films around soil particles, and;

4. Zero shrinkage; the clay volume does not decrease any further and water loss is equal to the increase of the air volume in the clay.

In this chapter we quantify the shrinkage of clay aggregates from three fields in the Purmer area, approximately $15 \mathrm{~km}$ north of Amsterdam, the Netherlands. The Purmer area is a polder of $27.55 \mathrm{~km}^{2}$ with clay holding soils of marine origin, with an elevation between 3 and $4.5 \mathrm{~m}$ below mean sea level. The area is artificially drained by three pumping stations to maintain water levels suitable for agriculture. The study area in the Purmer is chosen, since in preliminary InSAR analysis phase changes between adjacent fields were observed. These could be caused by differential surface elevation changes resulting from swelling and shrinkage of the clayey soils, and therefore further investigation of this hypothesis is relevant for this thesis. Besides, the well-defined catchment area of the polder is advantageous for potential extension of the current research to a water balance study.

Quantification of shrinkage is done through determination of the grain size distribution, clay mineralogy, and laboratory measurements to construct the soil shrinkage 
characteristic curve, or short, soil shrinkage curve or shrinkage characteristic. Based on the modelling framework proposed by (Stewart et al., 2016), we will further analyse and characterise aggregate shrinkage and discuss implications for further use in modelling vertical shrinkage.

\subsection{Methods}

Soils at three adjacent fields in the Purmer area were sampled to determine grain size distributions, clay mineralogy, and shrinkage potential in terms of Soil Shrinkage Curve (SSC), Coefficient Of Linear Extensibility (COLE) and Potential Linear Extensibility (PLE). Soil samples for grain size distribution determination were taken in April and May 2010. Samples for shrinkage potential determination were taken at additional moments throughout 2011. Samples for shrinkage potential determination were actually soil slabs, from which aggregates for measurements were taken in the laboratory. Per field, 4 to 5 depths in the upper $100 \mathrm{~cm}$ of the soil were sampled, roughly: $0-10,15-25,30-40,50-60$, and 70-80 cm. A slight difference in sampling depths for grains size distribution determination and shrinkage potential determination has occurred due to different sampling moments and because determination of shrinkage potential of non-clayey layers has been omitted. Exact depth of sampled layers can be found in the results section for grain size distribution and soil shrinkage curve.

\subsubsection{Grain size distribution and clay mineralogy}

Grains size distribution was determined by laser diffraction as described by Buurman et al. (2001), using the Coulter LS230 instrument with fluid module. The analyses provide grain size distribution in volume percent from 0.04 to $2000 \mu \mathrm{m}$, and are summarised as the clay $(<2 \mu \mathrm{m})$, fine silts $(2--16 \mu \mathrm{m})$, medium to coarse silts $(2--50 \mu \mathrm{m})$, and sand $(>50 \mu \mathrm{m})$ fractions. Mineralogy of the clay fraction was determined once by X-Ray Diffraction (XRD), using a mix of samples.

\subsubsection{COLE and PLE}

The Coefficient Of Linear Extensibility (COLE) quantifies the lengthwise swelling and shrinkage potential of aggregates or soil layers (Grossman et al., 1968). For irregular shaped aggregates, COLE is based on volume measurements and defined as:

$$
\text { COLE }=\left(\frac{V_{w e t}}{V_{d r y}}\right)^{1 / 3}-1,
$$

where $V_{w e t}$ and $V_{d r y}$ are wet and dry aggregate volumes. $V_{w e t}$ and $V_{d r y}$ are defined as the volume at saturation and the volume at air-dryness, as this is the maximum moisture range that can be expected in a field soil under Dutch climatic conditions. Note that aggregates shrinkage is assumed to be isotropic, to define COLE as measure of linear extensibility (Grossman et al., 1968). COLE values can however be used to assess volume change potential, regardless of shrinkage geometry.

In the field, soils are often layered and for each layer, COLE values may differ. To assess Potential Linear Extensibility (PLE, extensibility in the vertical direction) of 
a field soil, COLE values per soil layer need to be taken into account. In this case, the assumption of isotropic shrinkage is relevant, and PLE is defined as (Bronswijk \& Evers-Vermeer, 1990):

$$
\mathrm{PLE}=\sum_{i=1}^{n} \mathrm{COLE}_{i} * z_{i},
$$

where $\mathrm{COLE}_{i}$ and $z_{i}$ are the COLE and thickness of $i$ th soil layer. Here, PLE is derived for the upper $100 \mathrm{~cm}$ of the soil, from COLE and layer depths of 4 to 5 layers.

\subsubsection{Soil shrinkage curve}

To determine the relation between aggregate volume and moisture content, the soil shrinkage curve was determined for each sampled layer. Soil samples (i.e. slabs) were saturated in the laboratory for 2 weeks by resting them in a layer of water, after which aggregates were taken from the samples. Per layer 4 to 8 aggregates were collected, with saturated volume between 10 and $43 \mathrm{~cm}^{3}$. Aggregates were coated with an elastic, semi-permeable coating (permeable to water vapour, impermeable to water upon short immersion) of a Polyvinylidene Chloride (PVDC) resin and solvent mixture (resin to solvent ratio 1:4) (Brasher et al., 1966; Bronswijk \& Evers-Vermeer, 1990). This allowed repeated measurement of aggregate weight and underwater weight while drying out. Underwater weight served as an accurate determination of aggregate (and intra-aggregate) volume. After volume loss upon air-drying under laboratory conditions became negligible (volume change less than $1 \%$ per week), the aggregates were oven-dried for 24 hours at $105^{\circ} \mathrm{C}$ twice and dry weight and dry volume were measured.

The soil shrinkage curve typically relates the volume of an aggregate or soil and its water content. Exact description of the soil shrinkage curve depends on the formulation of volume and water content. Aggregate volume can for example be expressed as the void ratio $e$ (volume of aggregate pores over volume of solids) or porosity $\phi$ (volume of aggregate pores over total aggregate volume), and moisture content can be expressed as the moisture ratio $\vartheta$ (volume of water over volume of solids) or gravimetric water content $u$ (mass of water over mass of solids). Using the measurement methodology as described above, provides actual and oven-dry weights and volumes of aggregates. Together with the bulk densities of water and solid particles (assumed to be $1.00 \mathrm{gcm}^{-3}$ and $2.65 \mathrm{gcm}^{-3}$ ), all these above mentioned variables can be calculated allowing flexibility in presentation and comparison with other data sets.

\subsection{Results and discussion}

\subsubsection{Grain size distribution and clay mineralogy}

Grain size distributions of several soil layers at the fields are summarised in Tab. 3.1. The top $50 \mathrm{~cm}$ of the soils have a relatively high clay content, yet all are still classified as loam and sandy loam. At field A and C layering is obvious with loamy sand and sand horizons below $50 \mathrm{~cm}$ depth, with minimum clay content of $3.4 \%$ at $90-100 \mathrm{~cm}$ depth on field C. Maximum clay content observed is $23.6 \%$ at $70-80 \mathrm{~cm}$ depth on 
field B.

The clay fraction of the mixed sample consisted of $65 \%$ montmorillonite, $25 \%$ illite and $10 \%$ kaolinite minerals. The relative abundance of montmorillonite indicates a large swell-shrink potential of the clay fraction.

\subsubsection{COLE and PLE}

Table 3.1 also presents the measured COLE-values for each soil layer. No correlation between clay content and COLE has been observed if all fields are considered, but correlation is clearer if field $\mathrm{A}$ and $\mathrm{C}$ are considered individually $\left(R^{2}\right.$ of 0.45 and 0.64, albeit with very different relations). COLE and PLE allow comparison of the shrinkage potential with other soils. Bronswijk \& Evers-Vermeer (1990) have reported COLE values for Dutch clay aggregates measured between pressure head $h=0 \mathrm{~cm}$ (saturation) and $h=-16000 \mathrm{~cm}$, which we here compare to COLE values between $h=0 \mathrm{~cm}$ and air dryness. Bronswijk reports COLE values between 0.048 and 0.199 in soils with 15.9 to $62.9 \%$ clay content, compared to COLE values in this study between 0.033 and 0.091 . The lower COLE values in this study is the effect of relatively low clay content, which is partly compensated by the presence of clay minerals with high intrinsic swell-shrink potential. This results in classification of the COLE values as medium to high (Parker et al., 1977). PLE values of the upper $100 \mathrm{~cm}$ of the soil, calculated from COLE values and layer thickness, at field A, B and C are 3.6, 5.0, and $6.8 \mathrm{~cm}$. The upper $100 \mathrm{~cm}$ is a realistic maximum depth to assess shrinkage potential for, as in a field situation in the study area depth of the vadose zone is limited due to constant high surface water and groundwater levels, and capillary rise that further prevents the soil from deep drying (see also Ch. 4). Based on the PLE values, the soils are classified as soils with low shrink-swell potential according to Bronswijk \& Evers-Vermeer (1990).

Table 3.1 Grain size distribution and COLE values at several depths on field A, B and C.

\begin{tabular}{ccccccc}
\hline Field & $\begin{array}{c}\text { Depth } \\
(\mathrm{cm})\end{array}$ & $\begin{array}{c}<2 \mu \mathrm{m} \\
(\%)\end{array}$ & $\begin{array}{c}2-16 \mu \mathrm{m} \\
(\%)\end{array}$ & $\begin{array}{c}16-50 \mu \mathrm{m} \\
(\%)\end{array}$ & $\begin{array}{c}>50 \mu \mathrm{m} \\
(\%)\end{array}$ & $\begin{array}{c}\text { COLE } \\
\text { mean (st.dev.) }\end{array}$ \\
\hline Field A & $0-10$ & 20.8 & 44.3 & 25.1 & 9.8 & $0.044(0.006)$ \\
& $15-25$ & 15.2 & 38.4 & 18.4 & 5.9 & $0.034(0.007)$ \\
& $40-50$ & 12.9 & 23.4 & 16.5 & 47.2 & $0.033(0.006)$ \\
Field B & $70-80$ & 7.7 & 12.6 & 6.4 & 73.3 & $0.036(0.011)^{a}$ \\
& $0-10$ & 19.0 & 40.1 & 20.8 & 21.1 & $0.054(0.012)$ \\
& $15-25$ & 18.0 & 35.1 & 23.5 & 23.4 & $0.064(0.003)$ \\
& $30-40$ & 19.8 & 34.8 & 22.7 & 22.7 & $0.036(0.003)$ \\
& $50-60$ & 18.2 & 33.9 & 21.7 & 26.2 & $0.038(0.008)$ \\
Field C & $70-80$ & 23.6 & 43.2 & 24.2 & 9.0 & $0.055(0.004)$ \\
& $0-15$ & 19.2 & 39.1 & 22.1 & 19.6 & $0.091(0.010)$ \\
& $15-30$ & 15.2 & 28.4 & 23.8 & 32.6 & $0.084(0.005)$ \\
& $30-50$ & 15.4 & 29.5 & 21.4 & 33.7 & $0.063(0.007)$ \\
& $50-70$ & 5.9 & 8.9 & 9.4 & 75.8 & $0.069(0.005)$ \\
& $90-100$ & 3.4 & 4.8 & 6.5 & 85.3 & $0.043(0.007)^{b}$ \\
\hline
\end{tabular}

\footnotetext{
${ }^{a}$ based on clods from $50-60 \mathrm{~cm}$ depth.

${ }^{b}$ based on clods from $70-80 \mathrm{~cm}$ depth.
} 


\subsubsection{Soil shrinkage curves}

Figure 3.1 presents the soil shrinkage curves at all fields as the relation between moisture ratio $\vartheta$ and void ratio $e$. At field A limited change in void ratio is observed. In other words; volume change of the aggregates over the observed moisture range is small. This could indicate limited shrinkage potential of the aggregates. It might also be that aggregates where not saturated at the start and part of the shrinkage has not been measured, as initial water content and porosity of the upper 2 layers of field A, were about $0.08 \mathrm{~cm}^{3} \mathrm{~cm}^{-3}$ smaller than at field $\mathrm{B}$ and $\mathrm{C}$. The change in void ratio is also larger at field $\mathrm{B}$ and $\mathrm{C}$, although the range remains modest if compared to shrinkage curves from e.g. Bronswijk \& Evers-Vermeer (1990). In terms of shrinkage phases, a relatively large normal shrinkage phase is observed in the upper parts of each field, especially in field C. For deeper layers, a separation of the shrinkage curve from the 1:1-line is present, indicating water loss without volume change in the early stage of drying, probably owing to a relatively large fraction of sand and associated structural pores in the aggregates. The immediate deviation from the 1:1-line indicates that large pores have not been completely filled at the start of the measurements, or were already drained during preparation of the aggregates. Therefore water loss in the structural shrinkage phase has not been measured. Only one aggregate at 50-60 cm of field $\mathrm{C}$ seems to exhibits a slight portion of structural shrinkage.
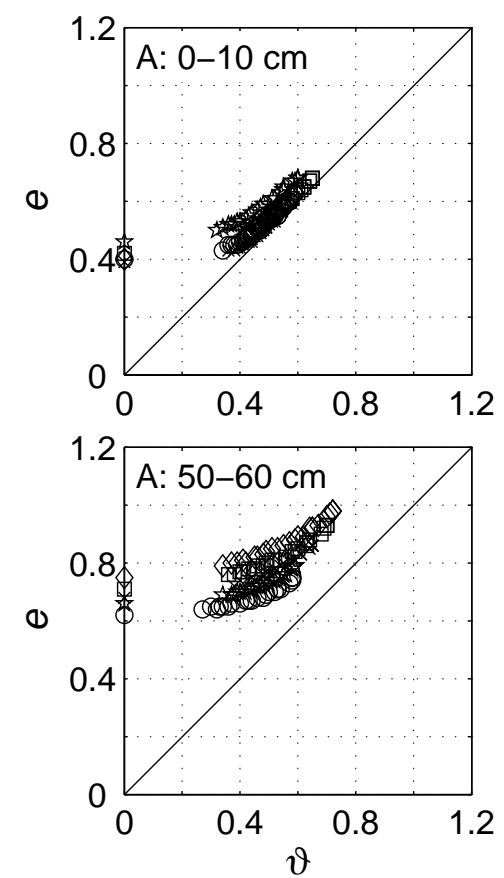

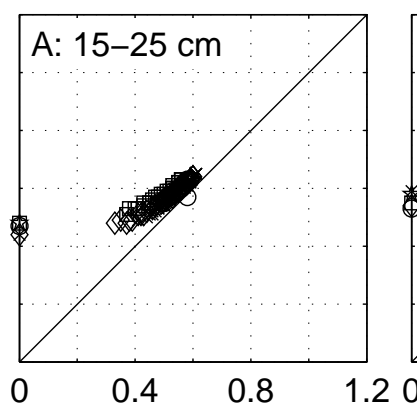

$\vartheta$

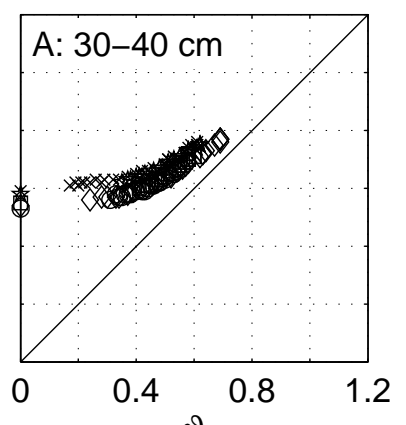

$\vartheta$

Fig. 3.1 Soil shrinkage curves of soil aggregates at several depths at field A. 

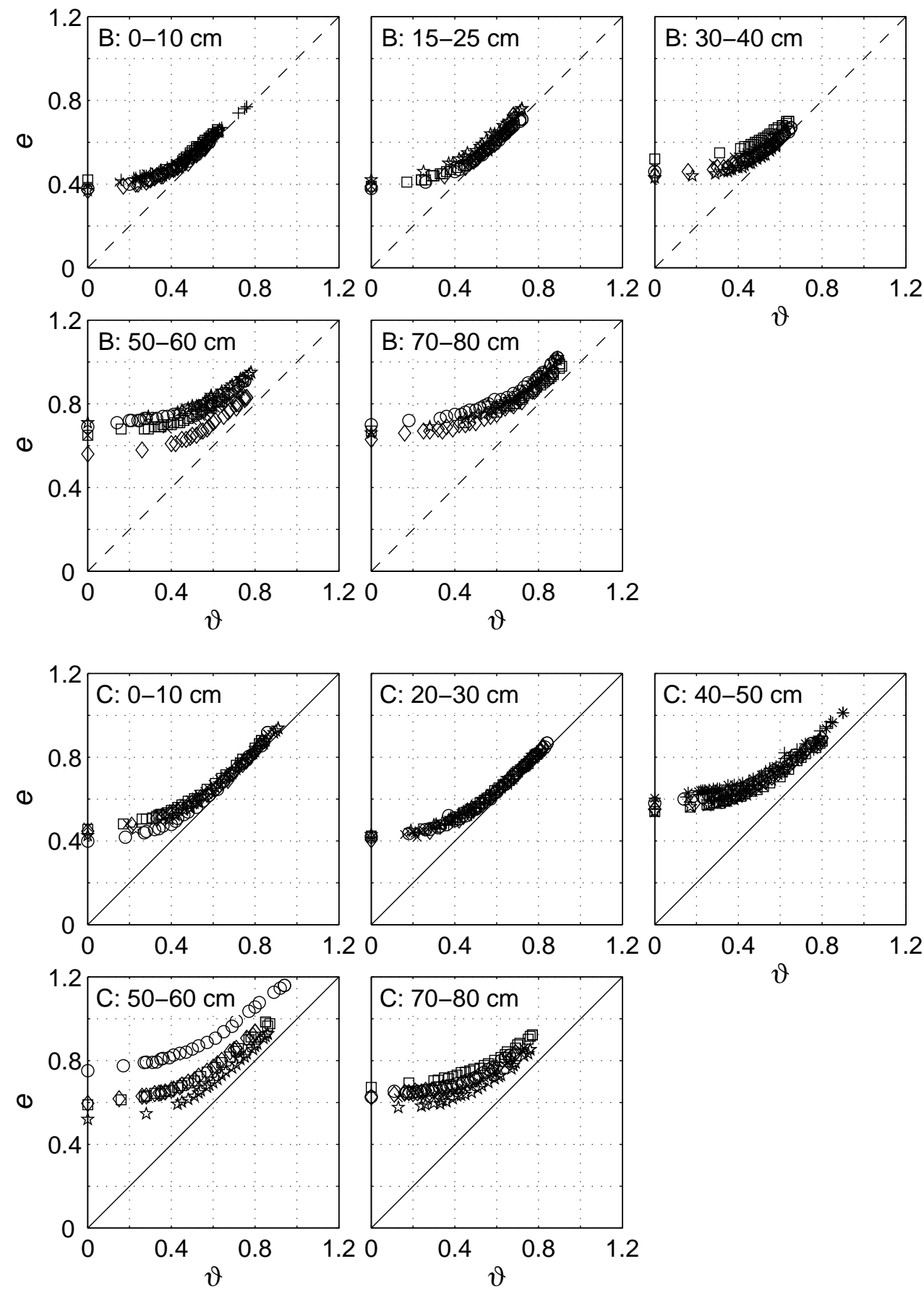

$\mathcal{v}$

Fig. 3.1 (continued) Soil shrinkage curves of soil aggregates at several depths at field B and C. 
The presence of structural pores in some aggregates could however explain the variation in shrinkage curves per layer (e.g. A: 50-60 cm, B: 50-60 cm, 70-80 cm, C: $50-60 \mathrm{~cm}, 70-80 \mathrm{~cm})$. In the dry end, shrinkage of almost all aggregates is characterised by residual shrinkage phase which seems to persist over a rather large moisture ratio range upon air drying, leading to hardly any measurement points in the zero shrinkage phase.

Another way of presenting the shrinkage curve is the relation between aggregate porosity and water content. Stewart et al. (2016) proposed a set of equations to describe this relation, including shifts of porosity between domains (aggregate porosity, subsidence porosity, and crack porosity), based on the aggregate shrinkage curve measurements and a model based on two fitting parameters. This set of equations enables also us to estimate vertical shrinkage (i.e. subsidence porosity) that can occur in a field soil. The shrinkage curve is defined as the relation between normalized water content $U$ and normalized porosity $\Phi$ :

$$
\begin{gathered}
U=\frac{u}{u_{\max }}, \\
\Phi=\frac{\phi_{\text {aggr }}-\phi_{\min }}{\phi_{\max }-\phi_{\min }} .
\end{gathered}
$$

The shrinkage curve is modelled by fitting a single continuous function (Stewart et al., 2016):

$$
\Phi_{\text {aggr }}(U)=\frac{(\epsilon+1) U^{q}}{1+\epsilon U^{q}}
$$

where $\epsilon$ and $q$ are fitting parameters. The obtained shrinkage curve and including $\epsilon$ and $q$ values after fitting are presented in Fig. 3.2. High values of $\epsilon$ indicate a large range of structural shrinkage, while low values of $q$ reflect limited zero and residual shrinkage (Stewart et al., 2016). Fitted values for field A are relatively high, both $\epsilon$ and $q$, indicating relatively large structural shrinkage and zero shrinkage. This thus also means that little normal shrinkage is to be expected. Shrinkage curves from Field B and C are rather comparable. For both field a distinction can be made between upper soil layers and lower soil layers based on $\epsilon$ value, with higher values in the lower soil and maximum value for field $\mathrm{C}$ at $50-60 \mathrm{~cm}$ depth. This corresponds to observations from Fig. 3.1. The values of $q$ are somewhat higher in field B than in field $\mathrm{C}$, but are rather constant in depth. The distinction between shrinkage phases can also be approximated with the second derivative of the soil shrinkage curve (Groenevelt \& Grant, 2001) and is provided by Stewart et al. (2016) for Eq. 3.5. The maximum of the second derivative reflects the water content $U$ at which structural shrinkage transitions to proportional shrinkage, while the minimum of this derivative indicates water content $U$ at the transition from proportional to residual shrinkage. The transition points are indicated in Fig. 3.2 as dashed vertical black lines. Due to the limited structural shrinkage observations the transition from structural to proportional shrinkage is almost immediate upon drying. The spacing between the vertical black lines indicates that aggregates experience normal shrinkage for approx. 40 to $50 \%$ of the moisture range. 

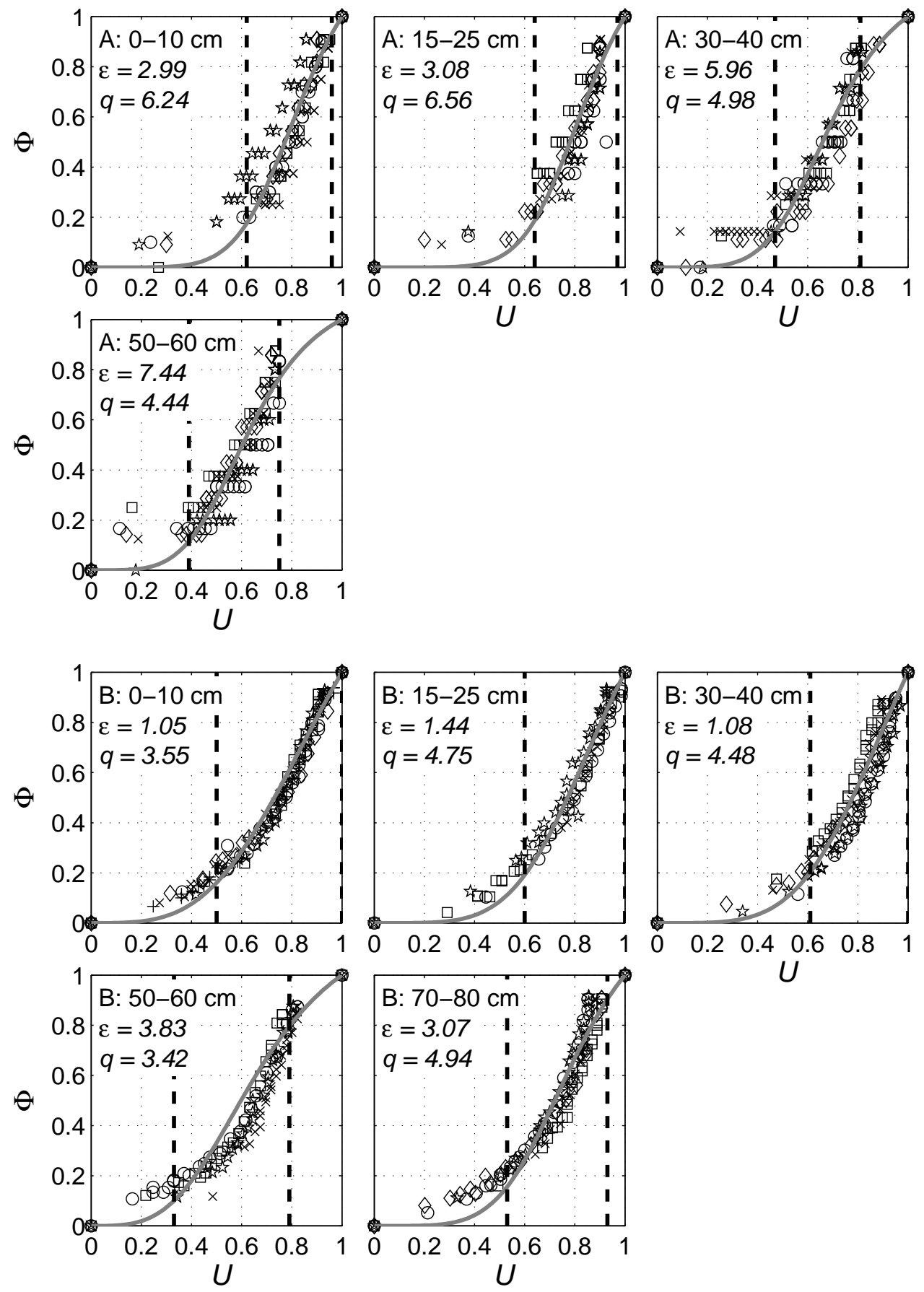

Fig. 3.2 Soil shrinkage curves of soil aggregates in terms of normalized water content $U$ and normalized porosity $\Phi$ at several depths at field A and B, with indication of obtained values for fitting parameters $\epsilon$ and $q$. 

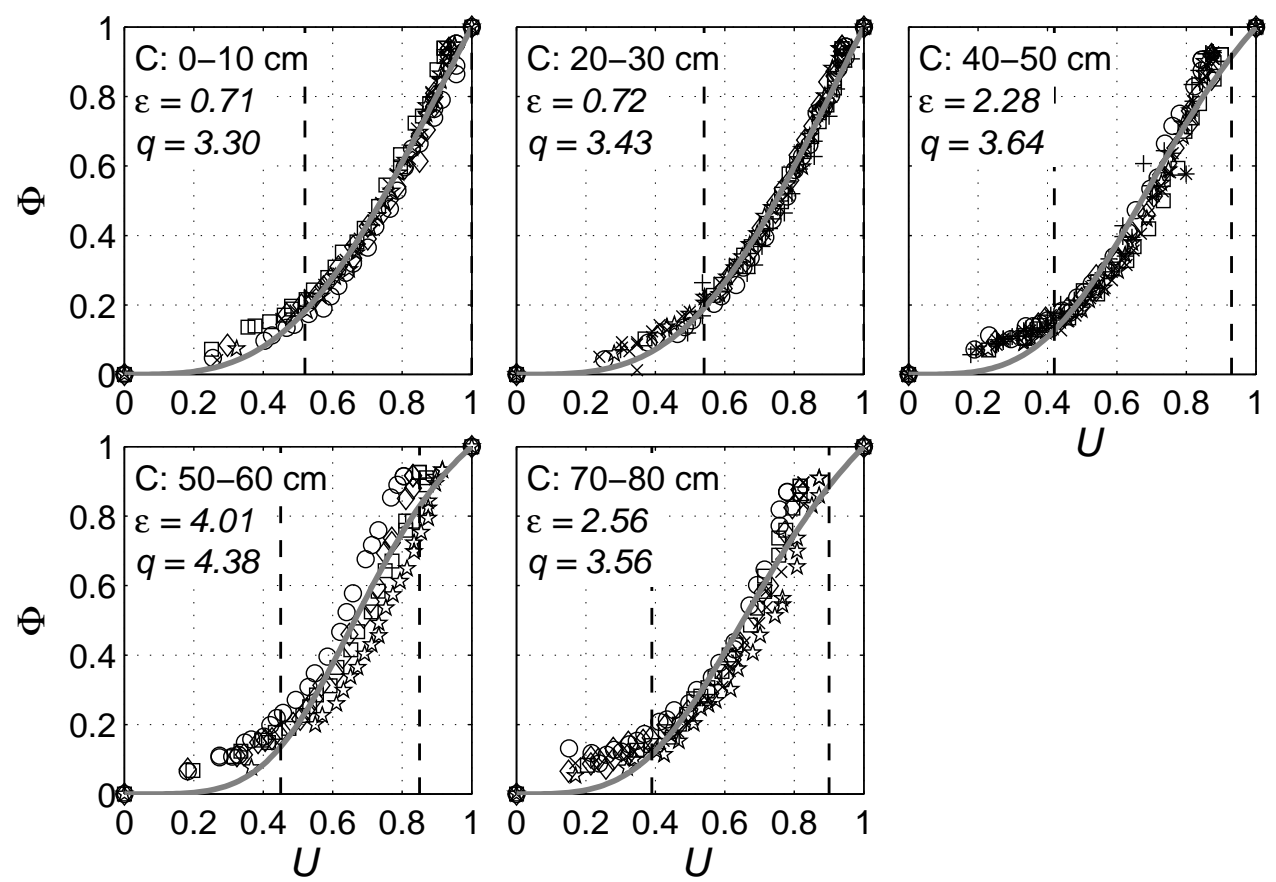

Fig. 3.2 (continued) Soil shrinkage curves of soil aggregates in terms of normalized water content $U$ and normalized porosity $\Phi$ at several depths at field $\mathrm{C}$, with indication of obtained values for fitting parameters $\epsilon$ and $q$.

\subsubsection{Subsidence modelling}

The obtained functions allow for modelling of crack porosity and subsidence porosity, as upon drying, aggregate porosity decreases and shifts to extra-aggregate porosity, consisting of subsidence and cracks.

Here, we compare subsidence porosity derived from the shrinkage curve with vertical shrinkage and volumetric soil moisture measurements CS616 sensors from (see Ch. 4) obtained at field B in spring 2011. At the location of vertical shrinkage measurements at field B, the clay content was somewhat higher than the clay content reported in Tab. 3.1 (25.9\%, compared to $19.0 \%$ ) We compare subsidence porosity and measured vertical shrinkage for two layers: $0-190 \mathrm{~mm}$ and $0-270 \mathrm{~mm}$, using shrinkage curves $B: 0-10 \mathrm{~cm}$ and $B: 15-25 \mathrm{~cm}$. The normalized ratio of layer thickness change $\Delta z$ over total layer thickness $z$ is equal to the normalized subsidence porosity. Stewart et al. (2016) derived expressions for subsidence porosity and the combined porosity of cracks and aggregates (i.e. pedon prososity). Using these derivations and the fitted shrinkage curves of aggregate porosity, we can derive the subsidence porosity for different shrinkage geometries, parametrised by the geometry factor $r_{\mathrm{s}}$.

The comparison between vertical shrinkage of the two layers of the field soil and modelled subsidence porosity is presented in Fig. 3.3. Modelled subsidence porosity 
based on the much used simplification (Bronswijk, 1990; Arnold et al., 2005; Stewart et al., 2016) of isotropic shrinkage (i.e. $r_{\mathrm{s}}=3$ ) is underestimated compared to vertical shrinkage measurements for both layers. The underestimation seems to be particularly large for low water contents as observed in the 0-190 $\mathrm{mm}$ layer. Subsidence modelling using adopted $r_{\mathrm{s}}$-values, matches the field measurements much better, as illustrated by the plotted lines for $r_{\mathrm{s}}=1.7$ and $r_{\mathrm{s}}=2$ in Fig. 3.3. Shrinkage with $1<r_{\mathrm{s}}<3$ is characterised by dominance of vertical shrinkage over cracking. As water content decreases the $r_{\mathrm{s}}$-value seems to get lower, indicating a dependence of shrinkage geometry on water content, as also observed by Braudeau et al. (1999), Chertkov et al. (2004), and Chertkov (2005). Based on the subsidence porosity model with non-isotropic shrinkage presented in Fig. 3.3, more than $60 \%$ of the aggregate porosity at saturation can shift to subsidence porosity upon drying. With an initial porosity of 0.42 , this means that vertical shrinkage of the soil layer of $27 \mathrm{~cm}$ could be up to $5.8 \mathrm{~cm}$. The higher clay percentage at the location of the vertical shrinkage measurements also contributes to the larger shrinkage. Still, the comparison between shrinkage curve and in situ measurements illustrates the potential to estimate shrinkage geometry.
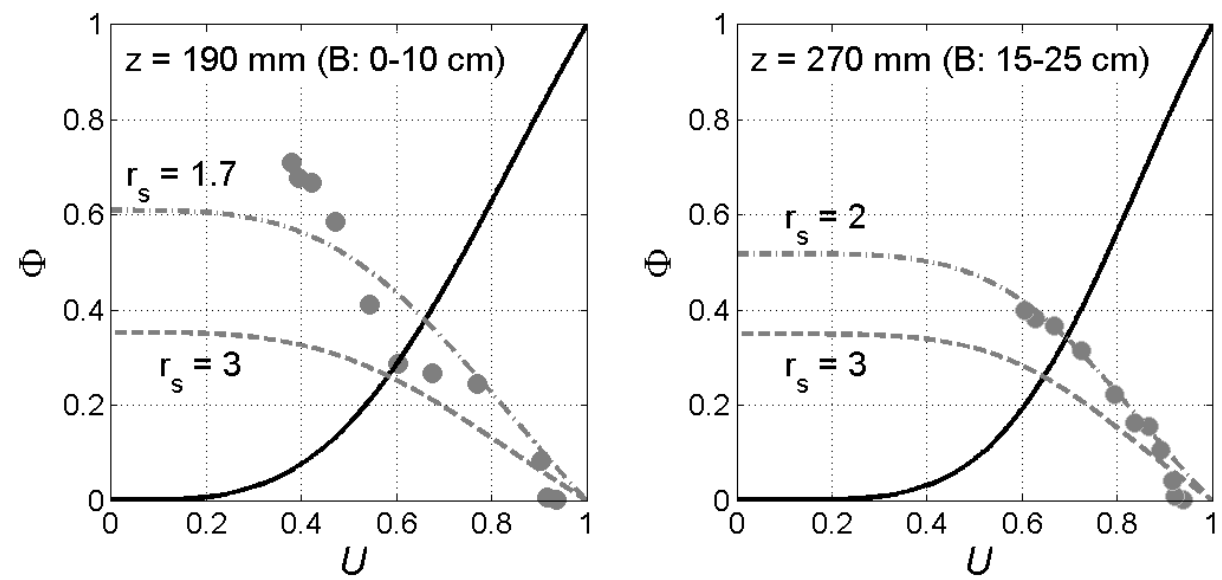

Fig. 3.3 Normalized aggregate and subsidence porosity against moisture content, based on shrinkage curve of field B, 0-10 cm (left) and 15-25 cm (right). Solid line is the fitted aggregate porosity from Fig. 3.2, and dashed lines are modelled subsidence porosities for isotropic shrinkage $\left(r_{\mathrm{s}}=3\right)$ and non-isotropic shrinkage $\left(r_{\mathrm{s}}<3\right)$. Grey points are data from Ch. 4 of layer thickness change over total layer thickness of $190 \mathrm{~mm}$ (left) and $270 \mathrm{~mm}$ (right) at various water contents, converted to normalized porosity.

\subsection{Conclusions}

We characterised shrinkage of clay aggregates from 3 field soils, to assess the potential of detecting surface level elevation changes. The relative abundance of montmorillonite, indicates that the clay fraction itself has high swell-shrink potential. Quan- 
tification of the potential through COLE also indicates medium to high shrinkage potential, while PLE values for the upper $100 \mathrm{~cm}$ of the soil indicate low to moderate potential. Also moderate swell-shrink potential is observed in the soil shrinkage curve. Structural shrinkage in some aggregates is promoted by large sand fractions, but limited observations have been done on water loss and volume change in the structural shrinkage phase due to non-complete saturation at the start of the experiments. Further quantification of the shrinkage curve, is performed through assessment of the porosity change with water content, as proposed by Stewart et al. (2016). The observation from the SSC are underlined by the fitting results for parameters $\epsilon$ and $q$, indicating larger structural shrinkage in aggregates with larger sand fractions. Computation of transitions points between shrinkage phases indicates that aggregates experience proportional shrinkage for approx. 40 to $50 \%$ of the moisture range. Modelled subsidence porosity based on fitted relations for aggregate porosity, shows a deviation with field data when isotropic shrinkage $\left(r_{\mathrm{s}}=3\right)$ is assumed. A lowering of the $r_{\mathrm{s}}$-value seems appropriate, indicating dominance of vertical deformation over cracking with over $60 \%$ of initial aggregate porosity shifting towards subsidence upon drying. The anisotropy of aggregate shrinkage also means that the potential of vertical shrinkage from COLE and PLE values are underestimated, as these are calculated from wet and dry aggregate volumes, assuming isotropic shrinkage. As vertical shrinkage seems to be dominant over horizontal shrinkage, higher surface elevation changes can be observed at similar drying than in the case of isotropic shrinkage. This leads to surface elevation changes potentially within the range that is observable by InSAR. 


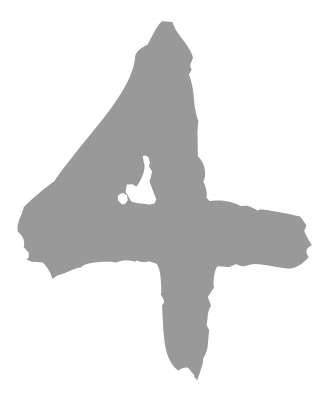

\section{Water storage change estimation from in situ shrinkage measurements of clay soils}

\subsection{Introduction}

The soil moisture status of the unsaturated zone has a major impact on terrestrial water fluxes. The amount and distribution of soil moisture determines the actual soil water storage capacity and the partitioning of precipitation into surface runoff, evaporation, transpiration, and groundwater recharge (Milly, 1994; Western et al., 1999; Robinson et al., 2008). Quantifying these water fluxes is often done through establishing the water balance of a control volume under consideration (e.g. unsaturated zone of the soil, catchment or continent). At large spatial scales, approaches like simple bucket models, often with lumped storage variables, might be satisfactory to establish the water balance (Milly \& Dunne, 1994; Farmer et al., 2003). At finer spatial scales, or to study short term water balance dynamics, a more detailed representation of variations in fluxes and state variables is required (Eagleson, 1978b) and measurements of soil water content are needed for closing the water balance (Robinson et al., 2008).

Methods to quantify soil water storage at and beyond the field scale are limited. Water balance methods have limited potential to determine soil water storage, as it is even harder to determine the various fluxes into and from the soil profile. The accumulation of measurement errors can be profound (Gee \& Hillel, 1988; De Vries \&

\footnotetext{
This chapter is published as Te Brake, B., Van der Ploeg, M.J., and De Rooij, G.H.: Water storage change estimation from in situ shrinkage measurements of clay soils. Hydrol. Earth Syst. Sci., 17, 1933-1949, 2013. doi:10.5194/hess-17-1933-2013.
} 
Simmers, 2002). In general, soil water storage is estimated from multiple soil water content measurements. Contact-based soil moisture sensors provide direct information with high frequency, but only on a very small measurement volume compared to the soil body of interest. To improve spatial coverage and reduce measurement errors, wireless sensors networks appear promising (Cardell-Oliver et al., 2005; Bogena et al., 2010). Optimally designing these networks for non-scientific applications still requires further work (Vereecken et al., 2008), but efforts in multiple disciplines, such as hardware technology, signal transmission, sensor data collection and data management, have resulted in significant progress in recent years (e.g. Bogena et al., 2007, 2009, 2010; Yang et al., 2010; Zhang et al., 2011). Contact-free measurements of soil moisture, such as ground based, airborne or spaceborne remote sensing techniques or hydrogeophysical measurements like ground penetrating radar and electromagnetic induction, can also provide information on larger spatial scales. The relatively low temporal resolution and complexity of data acquisition and processing of these measurements is a drawback. Besides, radiometer-operating remote sensing techniques suffer from limited penetration depth of the electromagnetic signal, resulting in a estimation of soil moisture content from the top few centimetres of the soil only (Wagner et al., 2007). Other applicability issues for these methods are quantification of the dielectric permittivity - soil moisture relation and surface roughness ambiguity (Huisman et al., 2003; Lievens et al., 2011). Modelling attempts to derive the soil profile water content from remotely sensed surface soil moisture measurements have only been partly successful (e.g. Arya et al., 1983; Walker et al., 2001), as factors like hydraulic parametrization, accuracy of surface soil moisture data, model simplifications and measurement frequency are often limiting factors (Vereecken et al., 2008). Also techniques to assimilate remotely sensed near-surface soil moisture observations into hydrological models require more development to explore all acquired data to its fullest (Crow \& Ryu, 2009; Liu et al., 2011; Draper et al., 2012).

The lack of fully applicable measurement techniques makes it desirable to develop an alternative methodology to measure soil profile water storage and subsequently quantify subsurface fluxes more accurately. Relying on relationships between soil water content and other, more easily and accurately measurable, variables to infer soil water storage from might be a possibility to do so. It has been long recognized that surface elevation changes of expansive clay soils could serve as an estimate for soil water storage change (Yule \& Ritchie, 1980a,b; Bronswijk, 1991a; Cabidoche \& Ozier-Lafontaine, 1995; Cabidoche \& Voltz, 1995; Kirby et al., 2003). Water storage change in clay soils results in volume change of the soil matrix and the relation between water storage change and volume change can be accurately quantified under laboratory conditions (e.g. Stirk, 1954; Bronswijk \& Evers-Vermeer, 1990; Braudeau et al., 1999; Cornelis et al., 2006), in lysimeters (e.g. Yule \& Ritchie, 1980a; Bronswijk, 1991b; Mitchell \& Van Genuchten, 1992) and in situ (e.g. Aitchison \& Holmes, 1953; Bridge \& Ross, 1984; Bronswijk, 1991a; Cabidoche \& Ozier-Lafontaine, 1995; Coquet et al., 1998; Kirby et al., 2003). Measuring change in total water stored in the soil rather than the vertical distribution of soil water is an acceptable simplification for many hydrological purposes. Therefore, volume change of clayey soils is an attractive proxy for water storage change. 
The shrinkage curve quantifies the relation between volume and water content of a volume under consideration. In the shrinkage curve, volume and water content are generally expressed relative to the volume of solids, resulting in dimensionless factors void ratio $e$ and moisture ratio $\vartheta$ :

$$
\begin{aligned}
& e=\frac{\text { Volume of pores }}{\text { Volume of solids }} \\
& \vartheta=\frac{\text { Volume of water }}{\text { Volume of solids }} .
\end{aligned}
$$

During shrinkage of initially saturated pure clay, the only mineral soil component that can cause shrinkage, three shrinkage phases can be distinguished. In the case of only external shrinkage, so that cracks do not develop in the clay, the shrinkage phases can be qualitatively described as (Haines, 1923; Bronswijk \& Evers-Vermeer, 1990; Chertkov, 2007a):

1. Normal shrinkage: water loss of the clay is completely compensated by volume decrease until the air entry point is reached. The clay remains saturated.

2. Residual shrinkage: water loss exceeds the volume change of the clay, from the air-entry point to the shrinkage limit, at which point all water resides in isolated pockets or in films around soil particles.

3. Zero shrinkage: the clay particles have reached their densest configuration. The clay volume does not decrease any further and water loss is equal to the increase of the air volume in the clay.

An additional shrinkage phase is often considered for shrinkage of aggregated soils (large samples comprising aggregates or field soils) that lose water upon first drying with limited, and often non-linear and non-constant, volume change (Stirk, 1954; Bronswijk \& Evers-Vermeer, 1990; Braudeau et al., 2004; Chertkov, 2007a). Water loss in this phase might originate from a relatively rigid soil textural domain (Chertkov, 2007a), relatively stable macro- or micro-pores, or from simultaneous drying of stable domains or pores and shrinking of inter-aggregates spaces (Braudeau \& Mohtar, 2006). The shrinkage curves of pure clay and that of an aggregated soil containing the clay also differ largely outside the structural shrinkage phase as a result of inter- and intra-aggregate crack development. Factors affecting crack development are measurement volume, sample preparation, clay content and soil structure (Jayawardane \& Greacen, 1987; Cornelis et al., 2006; Chertkov, 2007a,b, 2012a). As a result, the void ratio at a certain moisture ratio of an aggregated soil will never exceed the void ratio of a pure clay at that moisture ratio. The normal shrinkage phase remains characterised by a linear and constant decrease of the total volume with water loss, but water loss is essentially larger than volume decrease and shrinkage curve slopes will be less than unity (Jayawardane \& Greacen, 1987; Chertkov, 2007b). Therefore basic shrinkage is preferred terminology for a phase with a linear and constant decrease of volume with water loss. Figure 4.1 illustrates a shrinkage curve including all four shrinkage phases.

A soil experiencing multiple drying and wetting cycles, may exhibit the effect of hysteresis between swelling and shrinkage. This was observed by Peng \& Horn (2007) 


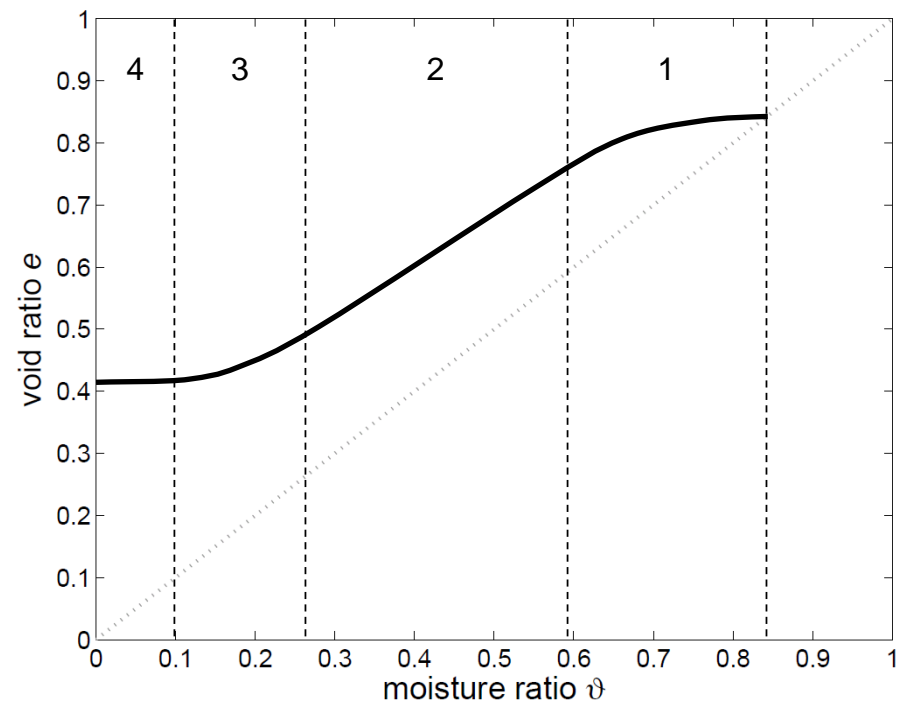

Fig. 4.1 Theoretical soil shrinkage curve, including 4 shrinkage phases: 1) structural shrinkage, 2) basic shrinkage, 3) residual shrinkage, 4) zero shrinkage.

after gradual drying and re-wetting of small cores. They distinguished two distinct parts in the swelling curve: virgin swelling at first swelling, with slopes close to one, followed by residual swelling at further wetting, where the moisture ratio increased but hardly any swelling was observed. According to Chertkov (2012b), the slope at first swelling can be larger or smaller than unity, depending on clay content, clay type and the moisture and void ratio at initial swelling. The occurrence of wetting stages during which the soil volume is not completely restored by swelling, may therefore result in an underestimation of volume change with respect to soil water storage change, and the slope of the relationship between volume change and soil water content change will be smaller than unity.

In situ measurements of the soil shrinkage curve are practically impossible to obtain, because crack volume can not be measured accurately. Therefore many authors (Aitchison \& Holmes, 1953; Bronswijk, 1991a; Mitchell, 1991; Cabidoche \& OzierLafontaine, 1995; Coquet et al., 1998; Kirby et al., 2003) relied on layer thickness measurements to calculate volume changes. Bronswijk (1991a) provided an equation to convert vertical shrinkage measurements to soil volume changes per unit area, based on model shrinkage of a cube of clay, experiencing only external shrinkage:

$$
\Delta V=\left[1-\left(1-\frac{\Delta z}{z}\right)^{r_{\mathrm{s}}}\right] z
$$

where $\Delta V$ is the volume change of the soil matrix per unit area, $\Delta z$ is the vertical layer thickness change and $z$ is the layer thickness at saturation, all in mm. $r_{\mathrm{s}}$ is the dimensionless shrinkage geometry factor (Rijniersce, 1983). In case of isotropic shrinkage, $r_{\mathrm{s}}=3$, while $1<r_{\mathrm{s}}<3$ indicates dominance of vertical shrinkage over cracking and $r_{\mathrm{s}}>3$ indicates dominance of cracking over vertical shrinkage. Bronswijk 
(1990) measured the shrinkage geometry of soil samples with and without overburden pressure, between two points at saturation and oven dryness. He concluded that removing overburden pressure yields $r_{\mathrm{s}}>3$, while including overburden pressure (as in a field situation) yields $r_{\mathrm{s}}=3$.

The model proposed by Bronswijk (1991a) is valid for structural and basic shrinkage and uses implicit assumptions when applied to soil layers:

1. A connected soil layer with distributed, not fully penetrating, cracks (connected soil layer) can be modelled as disconnected cubes. Therefore the distribution of volume change over thickness change and crack volume change of a connected soil layer is not influenced by horizontal stretching.

2. Cracks do not develop in drying soil samples (only external shrinkage).

3. The $r_{\mathrm{s}}$ factor does not vary with shrinkage phase and moisture content.

Chertkov et al. (2004) and Chertkov (2005) discussed the validity of these assumptions for the clay sample and soil layer case, and proposed correction factors for $r_{\mathrm{s}}$ variation with stretching, shrinkage phase, and soil moisture content, based on laboratory and in situ measurements of both vertical and horizontal shrinkage. The availability of the specific in situ data to calculate and test the applicability of these correction factors is limited, and hard to obtain.

The objective of this study is to test the applicability of the model by Bronswijk (1991a) and assumption of isotropic shrinkage at in situ overburden pressure (Bronswijk, 1990) to estimate volume change and soil water storage changes in the field from measurements of periodic vertical movement of the soil surface. We established the in situ relationship between soil water storage change from two types of contactbased sensors and soil volume change calculated from soil surface elevation changes, to test the validity of Bronswijk's simplified approach for soil layers of several depths. If this approach gives reasonable estimates of volume change of deep soil layers, we can reduce the dependence on laboratory measured shrinkage properties and in situ horizontal shrinkage measurements. This would then allow us to estimate changes in water storage in the soil profile from surface level elevation measurements. This may provide a basis for upscaling soil water storage change estimates to the field or catchment scale. The effect of geometry factor $r_{\mathrm{s}}$, drying regime, measurement scale of soil moisture sensors, profile depth and, texture variations in the soil profile are assessed.

\subsection{Materials and methods}

\subsubsection{Site description}

Field measurements of soil water content and vertical shrinkage were performed in the Purmer area, approximately $15 \mathrm{~km}$ north of Amsterdam, the Netherlands. The Purmer and its location in the Netherlands are shown in Fig. 4.2. Measurements were performed on two adjacent fields, labelled as field B and field C. On field B (Kentucky Bluegrass for seed harvesting) measurements were taken from April 2010 until October 2011. On field C (sugar beets) measurements were taken between April 2010 and November 2010. 


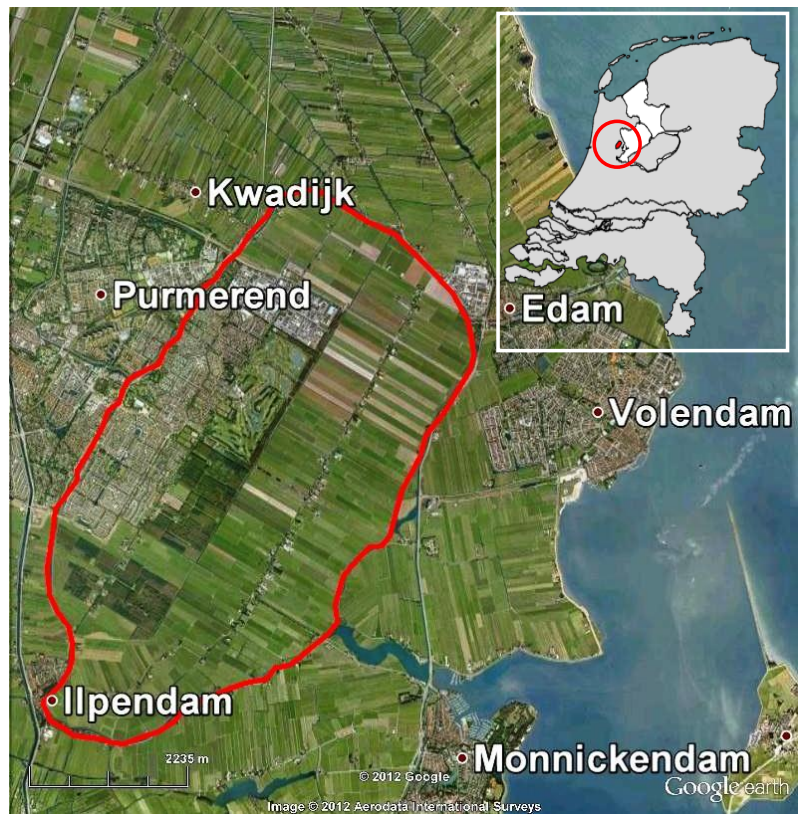

Fig. 4.2 Google Earth image of the measurement area and its location in the Netherlands. Map data: (C)2016 Google (https://www.google.com/earth/).

All soil layers in the upper $100 \mathrm{~cm}$ at field B were classified as loam (Soil Survey Staff, 2010). Below $100 \mathrm{~cm}$ a higher sand fraction was observed, but the exact grain size distribution was not determined. At field $\mathrm{C}$ the soil is clearly layered, with loam and sandy loam horizons in the upper $50 \mathrm{~cm}$ and loamy sand and sand horizons below (Tab. 4.1). The clay fraction in the area consisted of $65 \%$ montmorillonite, $25 \%$ illite and $10 \%$ kaolinite minerals, as determined by X-ray diffraction (see also Ch. 3 ).

\subsubsection{Swelling and shrinkage measurements}

To measure surface elevation changes resulting from clay swelling and shrinkage, ground anchors were installed based on a technique used by Bronswijk (1991a). The ground anchors consisted of metal rods with two $95 \mathrm{~mm}$ diameter discs at one end,

Table 4.1 Grain size distribution at several depths of soils at field B and field C.

\begin{tabular}{|c|c|c|c|c|c|c|c|c|}
\hline \multirow[b]{2}{*}{$\begin{array}{c}\text { Depth } \\
(\mathrm{cm})\end{array}$} & \multicolumn{4}{|c|}{ Field B } & \multicolumn{4}{|c|}{ Field C } \\
\hline & $\begin{array}{c}<2 \mu \mathrm{m} \\
(\%)\end{array}$ & $\begin{array}{c}2-16 \mu \mathrm{m} \\
(\%)\end{array}$ & $\begin{array}{c}16-50 \mu \mathrm{m} \\
(\%)\end{array}$ & $\begin{array}{c}>50 \mu \mathrm{m} \\
(\%)\end{array}$ & $\begin{array}{c}<2 \mu \mathrm{m} \\
(\%)\end{array}$ & $\begin{array}{c}2-16 \mu \mathrm{m} \\
(\%)\end{array}$ & $\begin{array}{c}16-50 \mu \mathrm{m} \\
(\%)\end{array}$ & $\begin{array}{c}>50 \mu \mathrm{m} \\
(\%)\end{array}$ \\
\hline $0-15$ & 19.0 & 40.1 & 20.8 & 21.1 & 19.2 & 39.1 & 22.1 & 19.6 \\
\hline $15-30$ & 18.0 & 35.1 & 23.5 & 23.4 & 15.2 & 28.4 & 23.8 & 32.6 \\
\hline $30-50$ & 19.8 & 34.8 & 22.7 & 22.7 & 15.4 & 29.5 & 21.4 & 33.7 \\
\hline $50-70$ & 18.2 & 33.9 & 21.7 & 26.2 & 5.9 & 8.9 & 9.4 & 75.8 \\
\hline $70-90$ & 23.6 & 43.2 & 24.2 & 9.0 & - & - & - & - \\
\hline $90-100$ & 18.9 & 35.6 & 19.1 & 26.4 & 3.4 & 4.8 & 6.5 & 85.3 \\
\hline
\end{tabular}


of which one could rotate freely and one was attached to the rod. When a ground anchor was lowered in a $100 \mathrm{~mm}$ diameter auger hole it was fixed by rotating the rod, forcing both discs into the undisturbed sides of the hole. After refilling the hole, a triangular frame was placed over the rod, resting on the undisturbed soil around the refilled hole on three pins (Fig. 4.3). The length of the rod above the triangular frame, $L$ (see Fig. 4.3), was measured between marked points on the triangular frame and at the top of the rod using a $0.01 \mathrm{~mm}$ accuracy digital calliper to record the change in thickness of the layer between the anchoring depth and the soil surface. In the following, the word "layer" refers to the soil slab between the soil surface and a given anchoring depth. Slabs of soil between two ground anchors are termed "layer increments". Anchoring depths were 11, 19, 29, 56, 92, and $152 \mathrm{~cm}$ at field B and $7,19,27,60,95$, and $157 \mathrm{~cm}$ at field C. For convenience we will refer to the targets depths of ground anchors $(10,20,30,60,100$, and $150 \mathrm{~cm}$ at each location) instead of exact layer thicknesses in the remainder of this paper. The measurement interval was mostly 11 days but ranged between 2 and 12 days for practical reasons. Cumulative thickness changes with respect to two reference days (15 May 2010 and 12 February 2011) were calculated.

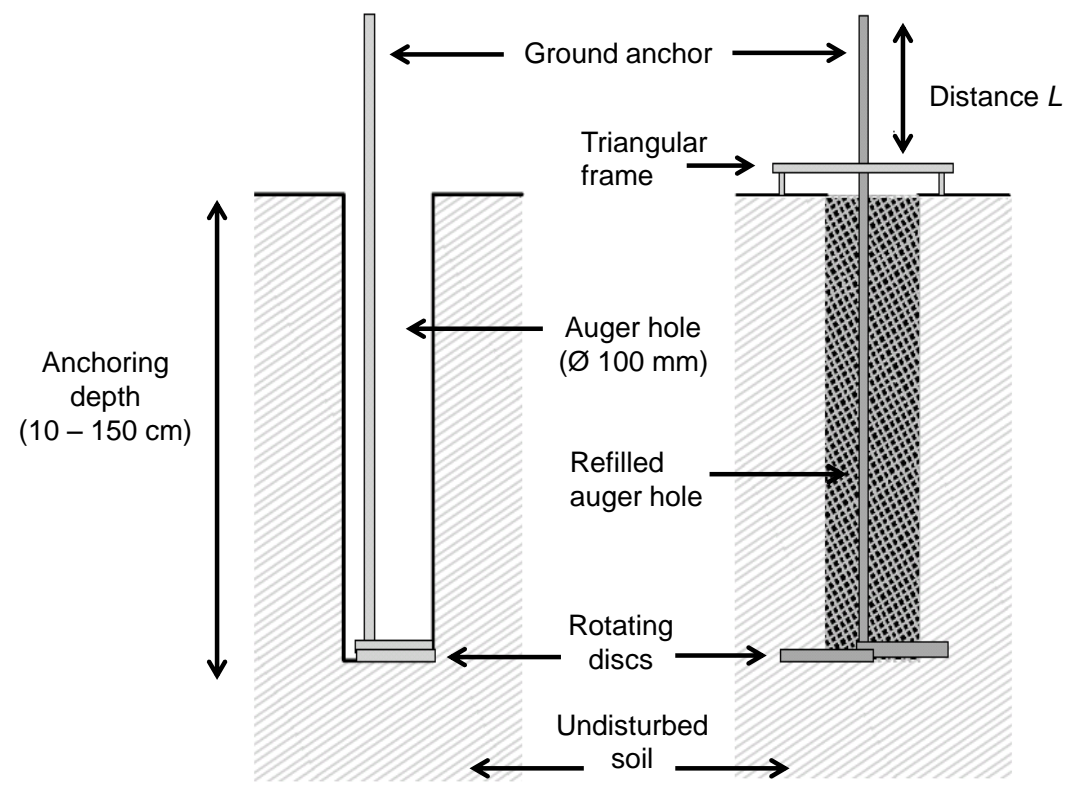

Fig. 4.3 Side view of the placement of ground anchors in the soil. Left: ground anchor lowered in auger hole. Right: ground anchor in refilled auger hole, with discs fixed in undisturbed soil after rotating the rod, and a metal frame placed over the rod. 


\subsubsection{Volume change}

Based on observations of $r_{\mathrm{s}}=3$ at in situ overburden pressure (Bronswijk, 1990), Eq. 4.3 was rewritten by Bronswijk (1991a) to calculated volume change of the soil matrix per unit area ( $\mathrm{mm})$ by:

$$
\Delta V=3 \Delta z-3 \frac{\Delta z^{2}}{z}+\frac{\Delta z^{3}}{z^{2}} .
$$

We used Eq. 4.4 to convert the layer thickness changes to volume changes of the soil pedon (excluding the volume of the cracks). Values for $z$ were substituted by $z(0)$, being the layer thicknesses at the reference day (either 15 May 2010 or 12 February 2011), at which the soil was assumed to be near saturation.

Bronswijk \& Evers-Vermeer (1990) estimated that Dutch clay soils, under Dutch climatic conditions, mainly experience basic shrinkage. For this situation $\Delta V$ equals water storage change $\Delta W$. Including $S$ for water loss in the structural shrinkage phase (Yule \& Ritchie, 1980a) yields:

$$
\Delta W=S+\Delta V
$$

\subsubsection{Soil moisture measurements}

Volumetric soil moisture content was measured with two contact-based sensor types: EC-5 capacitance sensors (Decagon) and CS616 water content reflectometers (Campbell Scientific). Both sensors measure the dielectric permittivity of the soil, from which volumetric moisture content is calculated. The high frequency of $70 \mathrm{MHz}$ at which both sensors are operating minimizes salinity and textural effects (Logsdon, 2009; Parsons \& Bandaranayake, 2009; Francesca et al., 2010).

EC-5s have two flat, $1 \times 5 \times 56 \mathrm{~mm}$ pins spaced $5 \mathrm{~mm}$ apart, while CS616s have two $300 \mathrm{~mm}$ long rods with a diameter of $3.2 \mathrm{~mm}$, spaced $32 \mathrm{~mm}$ apart. Measurement rods of CS616s are therefore almost 5 times longer and wider apart than those of EC-5s. As the measurement volume of EC-5s is restricted to the direct surroundings of the pins (Sakaki et al., 2008; Parsons \& Bandaranayake, 2009), it is far smaller than the measurement volume of the CS616s (Francesca et al., 2010).

The difference in measurement volume of the sensor types enabled us to study the relation between volume changes and soil water storage changes on two spatial scales. EC-5s were assumed to measure soil water content on intra-aggregate scales, while CS616s measured soil water content over a larger volume including multiple aggregates and inter-aggregate spaces (the bulk soil). Nine EC-5s were installed at 5, 7.5, 10, 22.5, 30, 45, 60, 80 and $100 \mathrm{~cm}$ depth, four CS616s at 7.5, 22.5, 45 and $80 \mathrm{~cm}$ depth. At field B, EC-5s were installed at 9 and $14 \mathrm{~cm}$ instead of 7.5 and $10 \mathrm{~cm}$, and a CS616 was installed at 9 instead of $7.5 \mathrm{~cm}$ depth. Sensors were horizontally installed in the undisturbed side of an installation pit. Their vertical alignment was slightly off so that each sensor had only undisturbed soil above and below it. The pit was carefully refilled after installation.

A custom calibration was carried out for the sensors. Soil samples were taken from the installation pits and dried and grounded to fill 5.5 L containers, with bulk densities 
approximating observed field soil bulk densities (between 1.26 and $1.50 \mathrm{~g} / \mathrm{cm}^{3}$ ). The soil in the containers was saturated with water and both EC-5 and CS616 sensors were installed. The soil was then left to dry at a constant temperature of $16^{\circ} \mathrm{C}$, with continuous monitoring of raw sensor output. Containers were weighted 80 times throughout the drying period to determine volumetric water content down to $0.05 \mathrm{~cm}^{3} \mathrm{~cm}^{-3}$. Water was assumed to have a density of $1.00 \mathrm{~g} / \mathrm{cm}^{3}$ and volume change of the clay was not taken into account. Estimated bulk density of soil clods (diameter approx. $3 \mathrm{~cm}$ ) at oven dryness ranged from 1.65 to $1.83 \mathrm{~g} / \mathrm{cm}^{3}$. Quadratic (EC-5) and cubic (CS616) calibration equations were fitted to volumetric water content and raw sensor output. Two different calibration equations were used for CS616s for soil layers with clay content $>15 \%$ and $<15 \%$. One calibration equation was used for EC-5s.

The daily averaged soil moisture content per sensor was calculated for days at which layer thickness changes were measured. Soil water storage $W$ was calculated twice for each layer, based on only EC-5 and only CS616 data, by assigning the mean soil moisture content of the closest sensor to any part of the layer under consideration. Because the thickness of the layers varied due to swelling and shrinkage, and $W$ was calculated based on the initial depth $d_{i}$ assigned to sensor $i$, we accounted for layer thickness change by the ratio between the actual layer thickness $z(t)$ and the initial layer thickness $z(0), f_{\text {cor }}$ :

$$
W(t)=\sum_{i=1}^{n} \theta_{i}(t) d_{i} \cdot f_{\text {cor }},
$$

with:

$$
f_{\text {cor }}=\frac{z_{l}(t)-z_{l-1}(t)}{z_{l}(0)-z_{l-1}(0)} .
$$

In Eq. 4.6, $n$ is the number of sensors used to calculate $W, \theta_{i}$ is the volumetric water content measured by sensor $i$ and $d_{i}$ is the depth assigned to this sensor. In Eq. 4.7, $l$ is the layer over which to calculate $W$ and $l-1$ is the layer between surface level and the preceding ground anchor. For example, to obtain the correction factor for the layer increment $30-60 \mathrm{~cm}, l$ is $0-60 \mathrm{~cm}$ and $l-1$ is $0-30 \mathrm{~cm} . z_{l}$ and $z_{l-1}$ denote the actual layer thicknesses at the time indicated in parentheses. Note that for the first layer $(0-10 \mathrm{~cm}), z_{l-1}$ cancels out and $f_{\text {cor }}$ is calculated from the ratio between actual layer thickness $z_{0-10}(t)$ and the initial layer thickness $z_{0-10}(0)$ only.

By applying this correction it was ensured that water storage in each sublayer was corrected proportionally to the thickness change of that sublayer and the correction was not lumped or averaged over the total layer under consideration. Soil water storage changes were then calculated with respect to the reference days. Due to sensor failures, the EC-5s at $45 \mathrm{~cm}$ and $100 \mathrm{~cm}$ at field B and the EC-5 at $45 \mathrm{~cm}$ at field $\mathrm{C}$ were not used in the calculations. 


\subsubsection{Groundwater level}

Pressure transducers recorded groundwater levels in piezometers next to the ground anchors. One piezometer was installed at each measurement location at 22 July 2010. Atmospheric pressure was measured at field B to correct the measurements by the pressure transducers in the piezometers.

\subsubsection{Meteorological data}

\section{Precipitation}

The rainfall rate was measured by a Parsivel disdrometer (OTT Hydrometry Ltd, extensively described by Yuter et al., 2006), located approximately $150 \mathrm{~m}$ from the measurement location at field $\mathrm{C}$ and $300 \mathrm{~m}$ from the measurement location at field $\mathrm{B}$. The disdrometer operated from June 2010 until October 2011, but due to datalogging problems, data between 7 July 2010 and 11 September 2010 were missing. Data gaps were filled with daily precipitation sums from the Royal Netherlands Meteorological Institute (KNMI, 2014) precipitation station in Edam, located approximately $2.7 \mathrm{~km}$ north-east of the field site. Readings between the disdrometer and the precipitation station showed no systematic differences. As daily precipitation sums from KNMI stations were measured between 08:00 and 08:00 UTC, the disdrometer recordings with a frequency of one minute were summed over the same interval.

\section{Potential evapotranspiration}

Daily values between 08:00 and 08:00 UTC of reference potential evapotranspiration from the KNMI weather station in Berkhout (approx. $16 \mathrm{~km}$ north of the field site) were used. KNMI used a modified Makkink method for calculation of reference potential evapotranspiration (De Bruin, 1987; De Bruin \& Lablans, 1998). Potential evapotranspiration for grass and sugar beets were calculated using crop coefficients per 10 day period provided by Feddes (1987). In the following, evapotranspiration is taken to mean potential evapotranspiration, unless stated differently.

\subsection{Results and discussion}

\subsubsection{Drying regime, soil shrinkage, and groundwater depth in 2011}

Spring 2011 was exceptionally drier, sunnier and warmer than average. KNMI (2014) reported the nation-wide averaged amount of precipitation $(49 \mathrm{~mm})$ in the months March, April and May to be the lowest in $100 \mathrm{yr}$. The total of sunshine hours $(686 \mathrm{~h})$ was the highest in $100 \mathrm{yr}$ time and mean temperature $\left(11^{\circ} \mathrm{C}\right)$ was the second highest ever recorded. Figure 4.4 shows the effect of these exceptional weather conditions on net precipitation, soil layer thickness change, soil moisture content, and groundwater depth at field B for the 112 day period under consideration here (12 February until 3 June 2011).

The period was characterised by progressive net evapotranspiration under meteorological forcing and the onset of the growing season. Total precipitation was $63.9 \mathrm{~mm}$. The precipitation event of late February had a substantial effect on cumulative $P$ ET, since the evapotranspiration rate was still small at that time (Fig. 4.4A). Later 

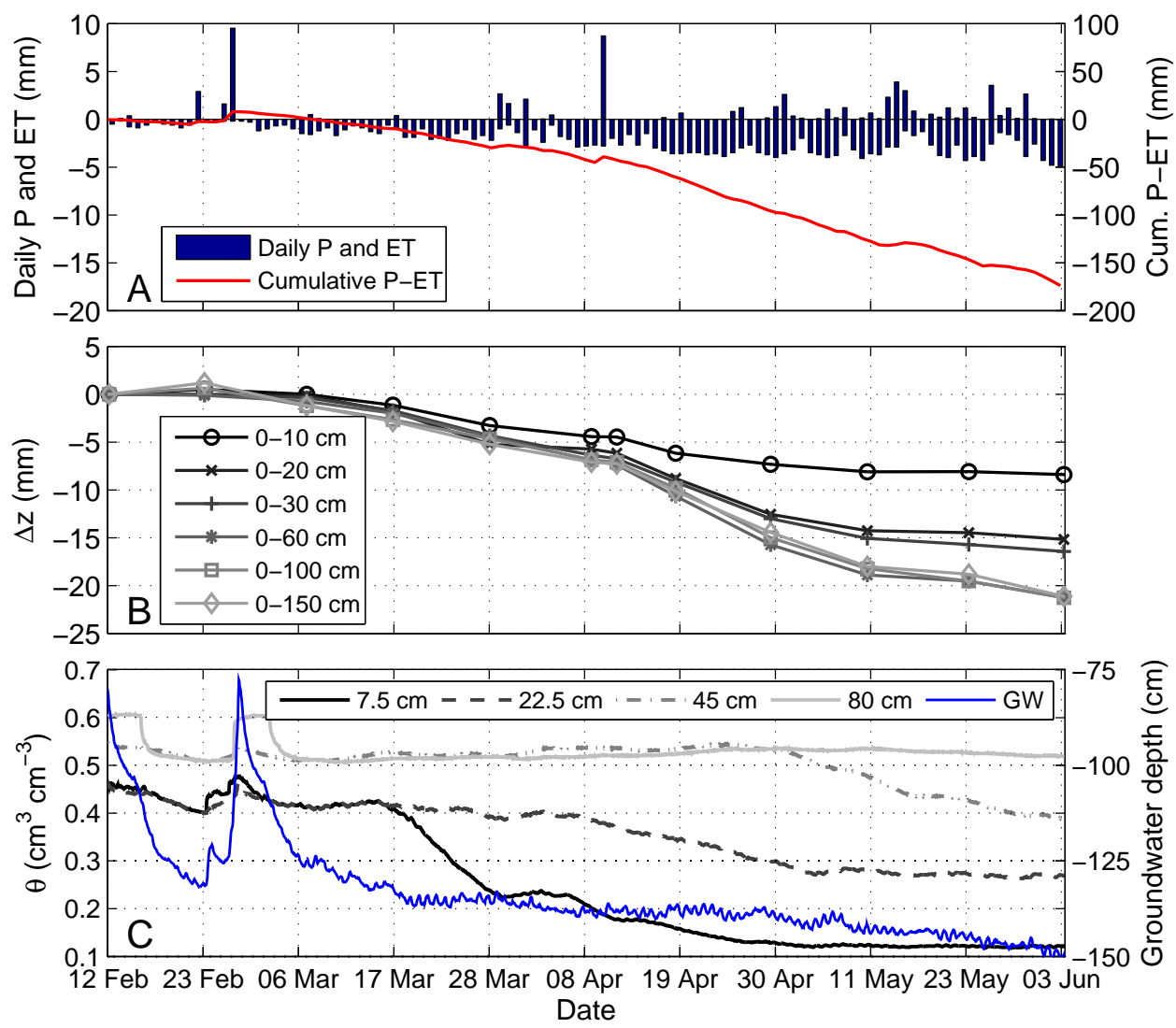

Fig. 4.4 Daily precipitation $P$, daily evapotranspiration $E T$ and cumulative net precipitation $P$-ET (A), cumulative layer thickness change $\Delta z$ in six soil layers (B), volumetric soil moisture content $\theta$ from CS616 sensors at four depths, and groundwater depth (C) at field B from 12 Feb. until 3 June 2011.

events were rapidly compensated by high evapotranspiration rates, resulting in total cumulative $P$-ET of $-174 \mathrm{~mm}$ at 3 June.

In February the soil was near saturation and the onset of the dry period at 28 February resulted in continuous shrinkage of all layers $(\Delta z)$ between 7 March and 3 June (Fig. 4.4B). The only exception is the layer $0-10 \mathrm{~cm}$ between 11 May and 23 May, where a negligible $0.02 \mathrm{~mm}$ swelling was observed. In the first interval between 12 February and 23 February no shrinkage was observed, but the layers $0-100 \mathrm{~cm}$ and $0-150 \mathrm{~cm}$ swelled up to $1 \mathrm{~mm}$. Total cumulative vertical shrinkage at 3 June in the layers $0-10,0-20,0-30$ and $0-60 \mathrm{~cm}$ was $8.4,15.2,16.4$ and $21.3 \mathrm{~mm}$. Shrinkage below $60 \mathrm{~cm}$ was negligible, as evidenced from the similarity of the curves below this depth. The layer increment between 30 and $60 \mathrm{~cm}$ started contributing significantly to total shrinkage at 19 April and shrinkage almost completely originated from this layer after 30 April.

At 1 May, the start of a decrease in soil moisture content $(\theta$, CS616) at $45 \mathrm{~cm}$ 
depth was measured (Fig. 4.4C), gradually proceeding to the end of the measurement period. In the same period, $\theta$ measured by sensors installed shallower and deeper than $45 \mathrm{~cm}$ remained relatively constant. This coincided with the dominance of shrinkage in the 30 to $60 \mathrm{~cm}$ layer increment. Small amounts of precipitation after 1 May did not increase the soil moisture content and no swelling was measured.

Although the groundwater level declined from approx. $100 \mathrm{~cm}$ below surface level in early March to approx. $150 \mathrm{~cm}$ in June (Fig. 4.4C), moisture content at $80 \mathrm{~cm}$ did not change in this period and no additional shrinkage was observed between 60 and $150 \mathrm{~cm}$. Around the time the groundwater level peaked twice in February however, the moisture content at $80 \mathrm{~cm}$ was changing abruptly. A time lag of about 4 days was observed between the decline of the groundwater level and soil moisture content. At the start of the soil moisture decline, the groundwater level was approx. $110 \mathrm{~cm}$ below surface level, indicating that the depth of the capillary fringe above groundwater level was approx. $30 \mathrm{~cm}$. Further lowering of the groundwater table and the capillary fringe caused $0.08 \mathrm{~cm}^{3} \mathrm{~cm}^{-3}$ moisture content change. Hardly any response of the aggregate-scale soil moisture content from EC-5s to groundwater fluctuations was observed. Relatively large pores emptied, while the soil matrix retained water. The observation of the depth and water content of the capillary fringe was used to correct water storage changes.

\subsubsection{Volume change and soil water storage change of a field soil during extensive drying}

Figure 4.5 shows volume change $\Delta V$ calculated from Eq. 4.4 and soil water storage change $\Delta W$ from the two water content sensor types in the six soil layers. For the soil layer extending from the soil surface to the capillary fringe, the $\Delta V-\Delta W$ relationship represents a soil profile scale shrinkage curve. A linear relation $(\Delta V=a \Delta W+b)$ was fitted through the data points representing volume change outside the structural shrinkage phase, meaning the first measurement interval was omitted, since no significant volume change was observed. According to this definition the decrease in water storage during the first interval is water loss in the structural shrinkage phase $S$. Fitted slope $a$ indicates the deviation from normal shrinkage, while correlation coefficient $R^{2}$ is a measure for the degree of basic shrinkage throughout the measurement period. Figure 4.5 gives $a$ and $R^{2}$, and all fitting parameters and goodness of fit $R^{2}$ are summarised in Table 4.2 .

Water loss in the structural shrinkage phase generally increased with depth (Tab. 4.2) and was larger for CS616 than for EC-5 measurements. This observation is consistent with the larger measurement volumes (comprising both aggregates and voids) of the CS616. The definition of structural shrinkage used here might be inaccurate for the deep layers $(0-100$ and $0-150 \mathrm{~cm})$, in which an initial stage spanning multiple measurement intervals with small volume change but large water storage change was followed by a large range of basic shrinkage as indicated by EC-5 data. This is in agreement with definitions by Chertkov (2007a) and observations by Braudeau et al. (1999).

All fitted slopes were smaller than one, as expected for a field soil. Slopes based on the two sensor types were comparable, with a maximum difference of 0.09 in the 

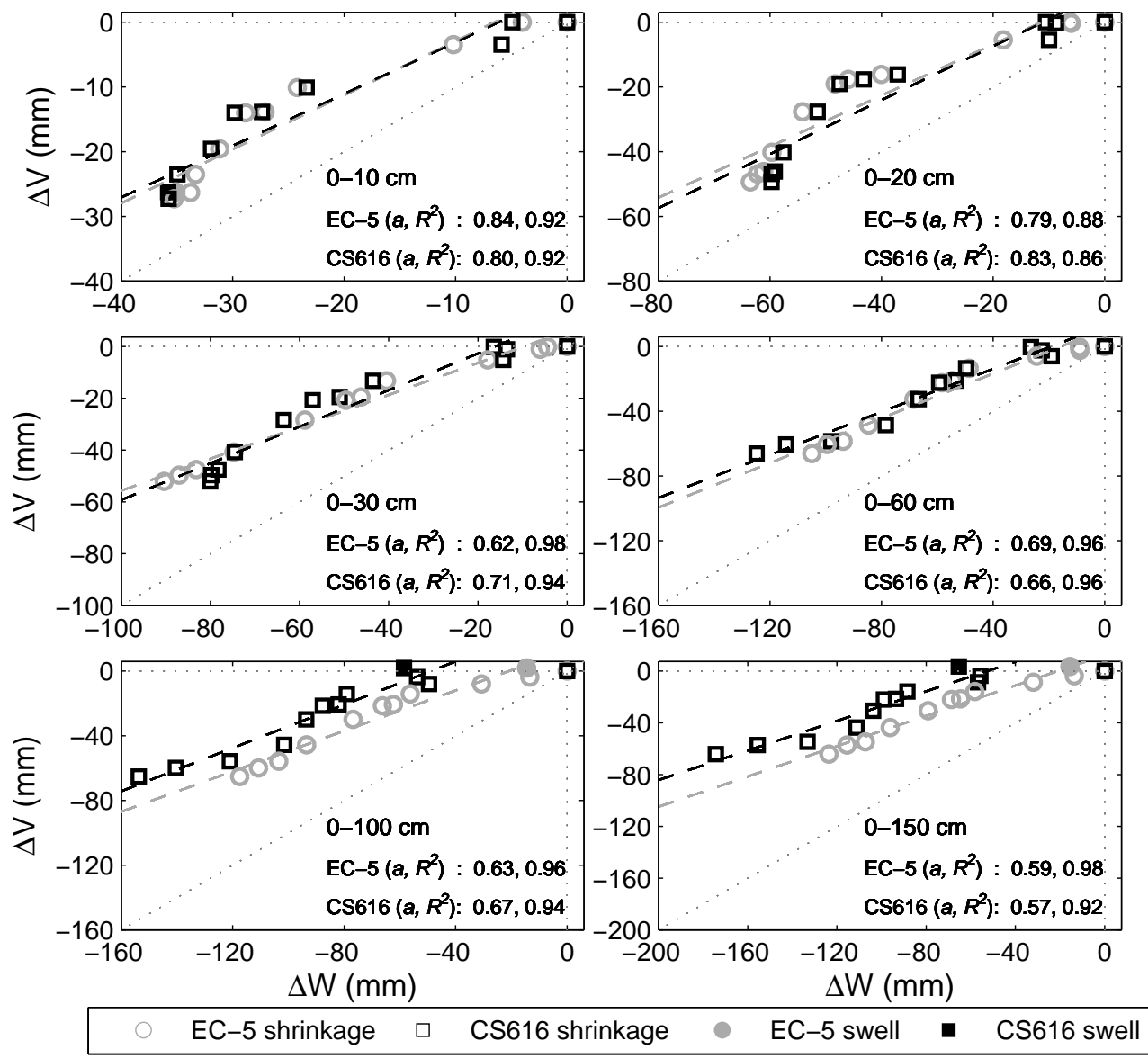

Fig. 4.5 Relationship between volume change per unit area $\Delta V$ and water storage change $\Delta W$ (EC-5 and CS616) at field B in 2011, for six soil layers. Dashed lines represent linear regression fits through data points outside the structural shrinkage phase, with $a$ indicating the slope of the fit and $R^{2}$ being the correlation coefficient.

0-30 cm layer. In this layer local slopes (slopes between two successive data points) larger than one are observed for the CS616 data (Fig. 4.5). Local slopes larger than one were also observed in the shallow layers $(0-10$ and $0-20 \mathrm{~cm})$, where volume change was overestimated compared to soil water storage change from both sensor types after an initial stage of considerable drying. When solely considering clay shrinkage, slopes larger than one are physically impossible. Relative overestimations of $\Delta V$ can result from an overestimation of the geometry factor $r_{\mathrm{s}}$ and therefore the assumption of constant $r_{\mathrm{s}}=3$, made by applying Eq. 4.4 seems invalid, at least in the dry range of the upper layers. Several authors (Bronswijk, 1988; Cornelis et al., 2006; Chertkov, $2005,2008)$ reported that $r_{\mathrm{s}}$ is a function of moisture content and shrinkage phase (Boivin, 2007).

Although no local slopes $>1$ were observed for layers deeper than $30 \mathrm{~cm}$, the 
Table 4.2 Fitted parameters for cumulative volume change outside the structural shrinkage phase, where $\Delta V=a \Delta W+b$, correlation coefficient $R^{2}$ between fit and observations, and observed water loss in the structural shrinkage phase $S_{\text {obs }}$ for 2011. Parameters $a^{\prime}, b^{\prime}$ and $R^{2^{\prime}}$ are fitted after applying $r_{\mathrm{s}}(\theta)$.

\begin{tabular}{ccccccccc}
\hline Sensor & Layer $(\mathrm{cm})$ & $a$ & $b$ & $R^{2}$ & $S_{\text {obs }}(\mathrm{mm})$ & $a^{\prime}$ & $b^{\prime}$ & $R^{2 \prime}$ \\
\hline \multirow{5}{*}{ EC-5 } & $0-10$ & 0.84 & 5.44 & 0.92 & 3.6 & 0.56 & 3.28 & 0.98 \\
& $0-20$ & 0.79 & 8.94 & 0.88 & 6.2 & 0.51 & 4.57 & 0.94 \\
& $0-30$ & 0.62 & 6.05 & 0.98 & 4.4 & 0.41 & 2.35 & 1.00 \\
& $0-60$ & 0.69 & 10.97 & 0.96 & 9.1 & 0.50 & 6.56 & 0.98 \\
& $0-100$ & 0.63 & 13.11 & 0.96 & 14.5 & 0.46 & 8.29 & 0.98 \\
CS616 & $0-150$ & 0.59 & 12.42 & 0.98 & 15.6 & 0.43 & 7.87 & 0.98 \\
\hline \multirow{5}{*}{} & $0-10$ & 0.80 & 4.90 & 0.92 & 5.8 & 0.53 & 2.65 & 0.96 \\
& $0-20$ & 0.83 & 9.18 & 0.86 & 10.5 & 0.55 & 4.63 & 0.94 \\
& $0-30$ & 0.71 & 11.55 & 0.94 & 16.5 & 0.48 & 6.25 & 0.98 \\
& $0-60$ & 0.66 & 12.90 & 0.96 & 26.5 & 0.48 & 7.69 & 0.96 \\
& $0-100$ & 0.67 & 32.84 & 0.94 & 58.5 & 0.49 & 22.47 & 0.94 \\
& $0-150$ & 0.57 & 30.27 & 0.92 & 65.4 & 0.42 & 20.69 & 0.92 \\
\hline
\end{tabular}

$r_{\mathrm{s}}$ overestimation in the upper layers also led to volume change overestimation in the deeper layers. To estimate possible errors introduced by assuming a constant geometry factor, $r_{\mathrm{s}}=3$, for deeper layers, a $r_{\mathrm{s}}$ range was linearly fitted to the observed moisture content range. A representative moisture content per measurement interval was obtained by averaging moisture contents at the $\Delta z$ measurement moments at the start and end of that interval. The moisture content range was 0.18 to $0.56 \mathrm{~cm}^{3} \mathrm{~cm}^{-3}$ $\mathrm{cm}^{3} \mathrm{~cm}^{-3}$ from EC-5s and 0.12 to $0.60 \mathrm{~cm}^{3} \mathrm{~cm}^{-3}$ from CS616s. In the first interval and at maximum moisture content $r_{\mathrm{s}}$ was assumed to be 3 , while at minimum moisture content $r_{\mathrm{s}}$ equalled a critical value at which the maximum of all local slopes (over a minimum of 4 datapoints) was 1 . Critical $r_{\mathrm{s}}$ values were 1.57 for EC-5s and 1.38 for CS616s.

The fitted $r_{\mathrm{s}}$ values per measurement interval and per layer increment were applied to calculated volume change in each layer by using:

$$
\Delta V_{l}=\Delta V_{l-1}+\left[1-\left(1-\frac{\Delta z_{l}-\Delta z_{l-1}}{z_{l}-z_{l-1}}\right)^{r_{\mathrm{s}} f i t}\right] z_{l}-z_{l-1},
$$

in line with Eq. 4.3. Here, $l$ is the layer of which to calculate $\Delta V, l-1$ is the layer between surface level and the preceding ground anchor (as in Eq. 4.7), and $r_{\mathrm{s}} f i t$ is the fitted $r_{\mathrm{s}}$ value. Note that for the first layer $(0-10 \mathrm{~cm}), z_{l-1}$ cancels out and $\Delta V$ is calculated using Eq. 4.3 with $r_{\mathrm{s}} f i t$ substituted.

By fitting $r_{\mathrm{s}}$ linearly to mean moisture content per layer increment and applying Eq. 4.8, effects of dry conditions in the upper parts of that layer were taken into account, and a realistic conservative $r_{\mathrm{s}}$ range was applied. Figure $4.6 \mathrm{~A}$ and $4.6 \mathrm{~B}$ show the results of linear fitting $r_{\mathrm{s}}$ to EC-5 and CS616 data per layer increment, obtaining the $r_{\mathrm{s}}$ fit values applied in Eq. 4.8. The effect of relatively high moisture content in the capillary fringe is clearly visible for the layer increments 30-60, 60 100 and $100-150 \mathrm{~cm}$. Figure $4.6 \mathrm{C}$ to $4.6 \mathrm{~F}$ illustrate how $r_{\mathrm{s}}$ fit propagated through time and depth. Figure $4.6 \mathrm{C}$ and $4.6 \mathrm{D}$ show the relationship between $\theta$ in the layer increments and the cumulative weighted average of $r_{\mathrm{s}}$ fit values over the length of 
the time intervals they were applied to. The obtained $r_{\mathrm{s}}$ values could be regarded as effective $r_{\mathrm{s}}$ values that could be applied in case of limited $\Delta z$ measurement moments. Figure $4.6 \mathrm{E}$ and $4.6 \mathrm{~F}$ show the relationship between $\theta$ in the total layers and the cumulative weighted average of $r_{\mathrm{s}}$ fit values over the length of the time intervals and the layer depths they were applied to. In this case the obtained $r_{\mathrm{s}}$ values could be regarded as effective $r_{\mathrm{s}}$ values that could be applied in case of limited $\Delta z$ measurement moments and a limited number of layers defined.
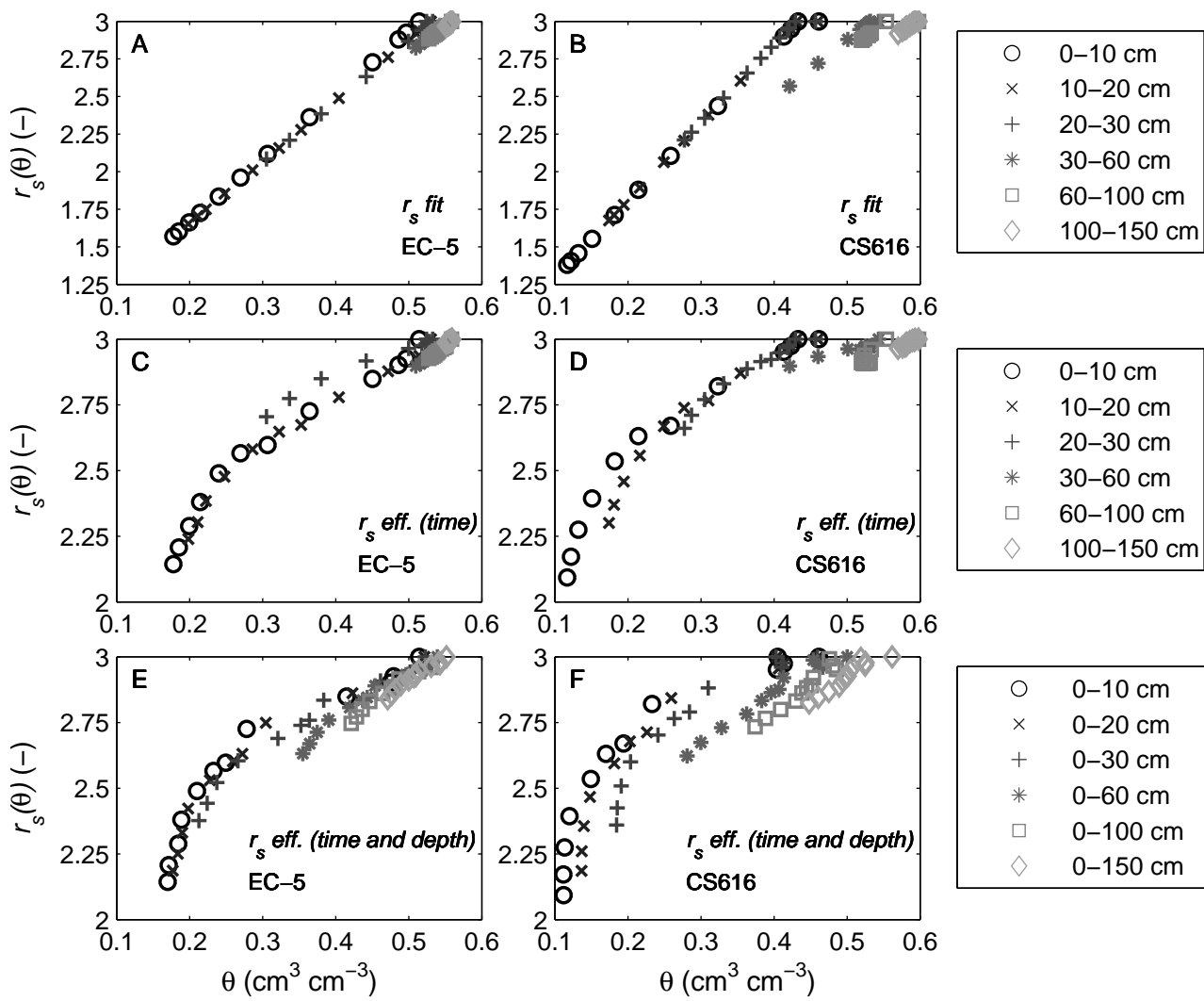

Fig. 4.6 Relationship between volumetric soil moisture content $\theta$ from EC-5 and CS616 and fitted $r_{\mathrm{s}}$ in layer increments (A and B), effective $r_{\mathrm{s}}$ over time in layer increments $(\mathrm{C}$ and $\mathrm{D})$, and effective $r_{\mathrm{s}}$ over time and depth of layers (E and $\left.\mathrm{F}\right)$.

Figure 4.7 shows the $\Delta V-\Delta W$ plot, after applying Eq. 4.8. Applied $r_{\mathrm{s}}$ range and fitting parameter per layer are summarised in Tab. 4.2. For all layers the EC-5 sensors captured soil moisture changes in aggregates that correlate with basic shrinkage, as evidenced from high $R^{2}$ values and relatively constant slopes. The CS616 sensors trended towards smaller slopes with depth for the 0-60, 0-100 and 0-150 cm layers, both in Fig. 4.5 and Fig. 4.7. Therefore, this can not be an effect of shrinkage 
geometry. In the penultimate measurement interval (between 11 May and 23 May), the volume change was only $2.3,3.3$ and $1.8 \mathrm{~mm}$ in the layers $0-60,0-100$ and 0 $150 \mathrm{~cm}$. At the same time soil water storage change (CS616) in these layers declined with $16.1,19.3$ and $22.3 \mathrm{~mm}$, resulting in local slopes of $0.14,0.17$ and 0.08 . The lower soil layers drained water stored in large pores to the lowered groundwater level (see Fig. 4.4C), which resulted in little shrinkage below $30 \mathrm{~cm}$ depth, but a rapid decrease in water storage. In the final measurement interval the local slopes for the layers 0-60, $0-100$ and $0-150 \mathrm{~cm}$ had increased again to $0.35,0.28$ and 0.27 . The fitted slopes $a$ for the deepest soil layers resulted from zero-shrinkage of the upper part of the soil, structural shrinkage of the lower part and residual shrinkage in the layers in between. This was the effect of soil moisture content differences in the profile, caused by delayed drying of the lower layers and large influence of the capillary fringe. Similar findings were reported by Yule \& Ritchie (1980a,b) for small and large cores. Yule \& Ritchie (1980b) suggested that simultaneous water loss from multiple depths in a profile may stem from the structural and basic shrinkage phase until most of the plant available water has been used.

The contribution of water loss in the structural shrinkage phase to the total water storage change in the profile is large. Initial water loss at structural shrinkage at the start of the measurement period $S_{o b s}$ for all layers is listed in Tab. 4.2. In the layers $0-60,0-100$ and $0-150 \mathrm{~cm}$ these amounts were amplified by water loss measured by CS616s in the two final measurement intervals. These water losses from delayed structural shrinkage were 26.9, 32.7 and $42.1 \mathrm{~mm}$. Water losses from the combined initial and delayed structural shrinkage in these layers were 53.4, 83.2 and $107.5 \mathrm{~mm}$, making up $42.8,54.1$ and $61.7 \%$ of total water losses in these layers over the entire measurement period.

\subsubsection{Effects of $r_{\mathrm{s}}$ variation on water storage change of the unsaturated zone}

The effect of lowered $r_{\mathrm{s}}$ values after fitting to the observed soil moisture contents, $r_{\mathrm{s}}(\theta)$, on $\Delta V$ is illustrated in Fig. 4.8 for the $0-20,0-60$ and $0-150 \mathrm{~cm}$ layers. Only $\Delta V$ based on CS616 data are shown here, since $\Delta V$ after using $r_{\mathrm{s}}(\theta)$ differed only slightly between CS616s and EC-5s (Fig. 4.7). The maximum difference in total volume change at 3 June calculated with $r_{\mathrm{s}}=3$ and $r_{\mathrm{s}}(\theta)$ was $17.7,17.5$ and $15.9 \mathrm{~mm}$ for the 0-20, 0-60 and 0-150 cm layers. This means that applying $r_{\mathrm{s}}(\theta)$ reduced total calculated volume change by $35.8,26.4$ and $24.8 \%$ with respect to using $r_{\mathrm{s}}=3$. Since we are primary interested in total soil water storage change of the unsaturated zone and hardly any volume change occurred below $60 \mathrm{~cm}$, we can illustrate the effect of $r_{\mathrm{s}}(\theta)$ on $\Delta W$ outside the initial structural shrinkage phase for for the 0-60 cm layer. Assuming a hypothetical slope of 0.5 for the $\Delta V-\Delta W$ relationship, the maximum overestimation of $\Delta W$ would be $35.0 \mathrm{~mm}$ when using $r_{\mathrm{s}}=3$, mainly stemming from the basic shrinkage phase. This would be $28.0 \%$ of total water storage change observed by CS616s in the $0-60 \mathrm{~cm}$ layer.

We assessed the minimum changes of geometry factor $r_{\mathrm{s}}$ with moisture content to prevent physically impossible slopes larger than unity. Fitted $r_{\mathrm{s}}(\theta)$ ranged from 3 to 1.38 between maximum and minimum in situ observed moisture content in the upper 

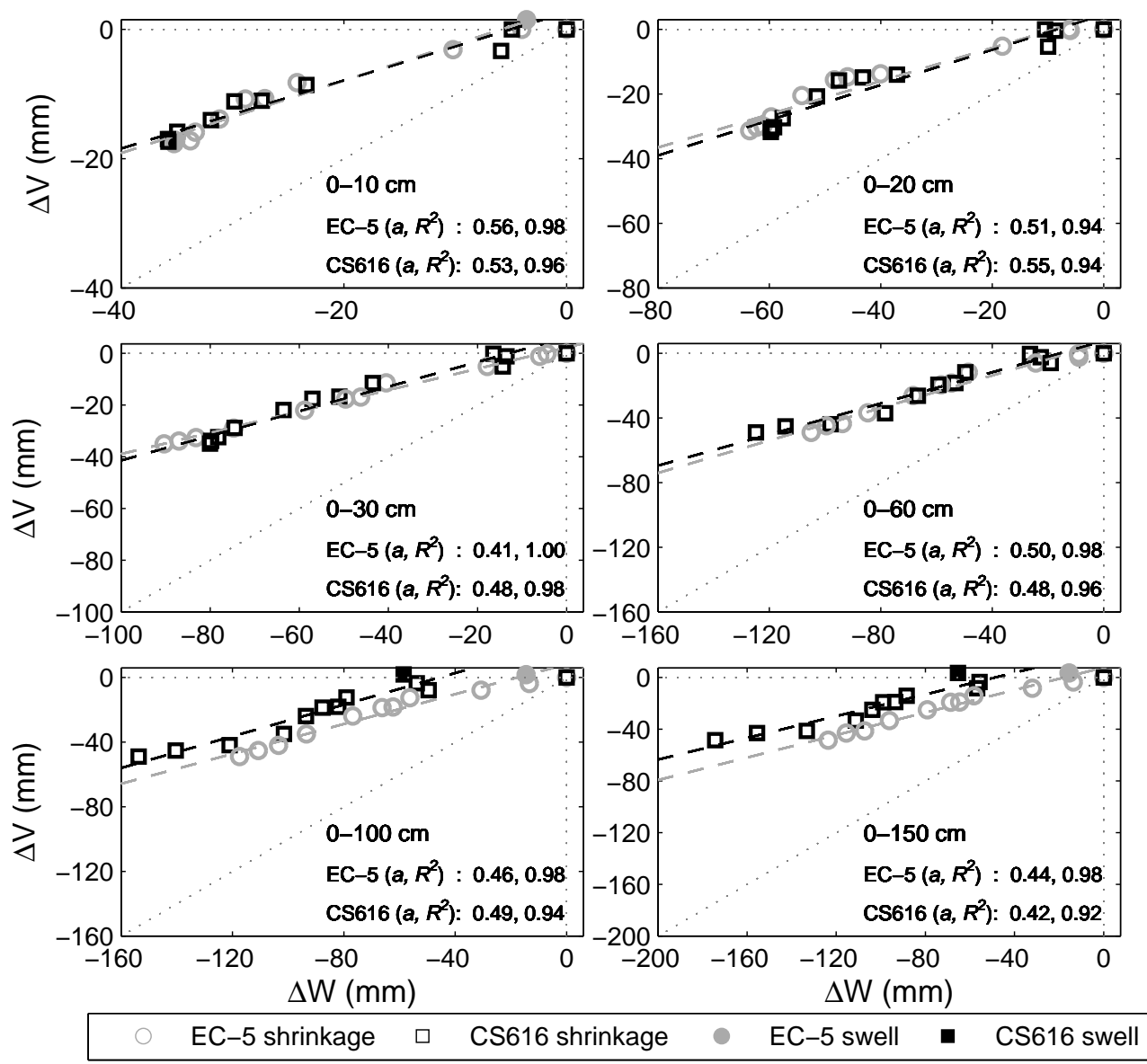

Fig. 4.7 As Fig. 4.5, but with $\Delta V$ calculated using fitted $r_{\mathrm{s}}$ factors and Eq. 4.8. Slope $a$ and correlation coefficient $R^{2}$ per layer are indicated.

layers of the soil (Tab. 4.2). Further lowering of $r_{\mathrm{s}}$, resulting in lower slopes of the $\Delta V-\Delta W$ relationship, therefore seems unnecessary and unrealistic.

The fitted $r_{\mathrm{s}}$ range and its linear decrease with moisture content seem in agreement with reports by Chertkov (2008, and Fig. 4 therein). By applying maximum $r_{\mathrm{s}}=3$ an underestimation of $\Delta V$ may have been introduced, as Chertkov (2008) estimated $r_{\mathrm{s}}>>3$, accounting for rapid crack formation in absence of vertical shrinkage at initial drying. We did not conclusively observe an initial increase of $r_{\mathrm{s}}$, but cannot rule out that this happened either. We decided not to take this into account in the $\Delta V$ calculations and set $r_{\mathrm{s}}=3$ for the first measurement interval.

The calculated effective $r_{\mathrm{s}}$ per layer (Fig. $4.6 \mathrm{E}$ and $4.6 \mathrm{~F}$ ) indicate that $r_{\mathrm{s}}$ should change with moisture content, but due to its purely empirical nature it is unsure if the calculated $r_{\mathrm{s}}-\theta$ relation can be transferred to other drying regimes/soils. To test this, $r_{\mathrm{s}}$ must be independently measured as function of moisture content to 


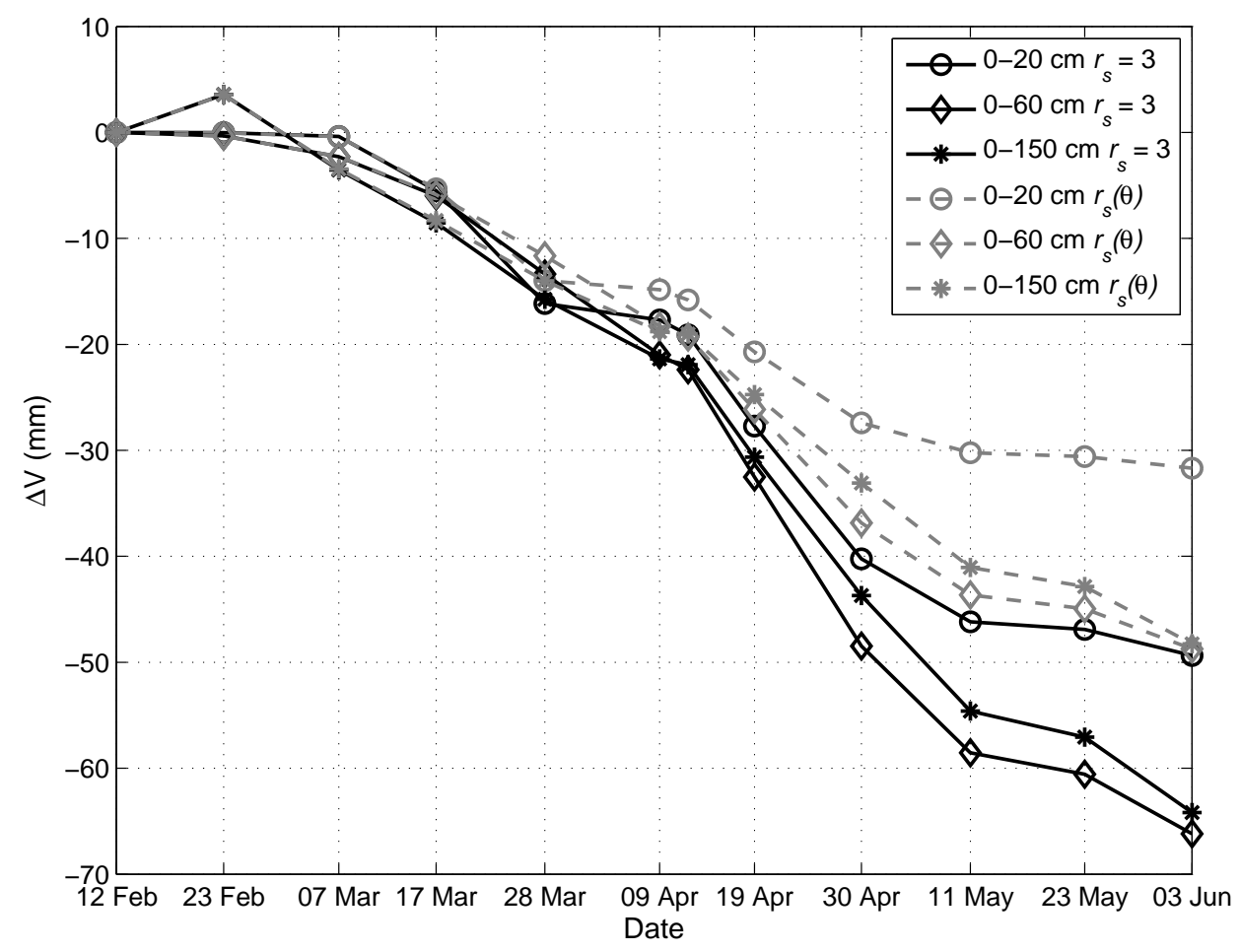

Fig. 4.8 Volume change $\Delta V$ at field $\mathrm{B}$ in 2011 for the layers $0-20,0-60$ and $0-150 \mathrm{~cm}$ for constant isotropic shrinkage $\left(r_{\mathrm{s}}=3\right)$ and shrinkage geometry as function of moisture content $r_{\mathrm{s}}(\theta)$.

accurately relate $\Delta V$ and $\Delta W$ when applied to Bronswijk's approach of measuring surface elevation changes and calculation of volume change at the appropriate scale. Corrections as proposed by Chertkov (2005) and Chertkov (2008) might be applied, but need specific data on shrinkage curves of clay and vertical and horizontal crack volume, which are hard to obtain non-destructively.

\subsubsection{Drying regime, soil shrinkage, and groundwater depth in 2010}

The growing season of 2010 offered a more dynamic precipitation and evapotranspiration regime, with both dry and wet periods, and measurements were performed on a field with a lower clay content (field C). The season was characterised by two periods of progressive drying, between 15 May and 8 June and between 11 June and 5 August, and a wet period after 5 August (Fig. 4.9A). The periods of net drying were separated by extensive rainfall $(49.1 \mathrm{~mm})$ on 9 and 10 June, resulting in swelling measured in all layers at 15 June (Fig. 4.9B). The second drying period included three days with a total rainfall of $69.6 \mathrm{~mm}$ in mid July. After 5 August, precipitation events were frequent and large, with a precipitation sum of $226 \mathrm{~mm}$ in August, while the 30 -yr mean total precipitation sum in August was $90 \mathrm{~mm}$.

At the start of the measurement period the soil was near saturation and soil layer thickness at the end of the measurement period was almost completely recovered 
to the level at the start. Between 15 June and 26 June, shrinkage of the $0-60 \mathrm{~cm}$ layer was larger than shrinkage of deeper layers. Magnitudes and patterns of thickness variations were comparable for the 0-30, 0-60 and 0-100 cm layers for other intervals. Most volume change originated from the upper $30 \mathrm{~cm}$ of the soil (Fig. 4.9B) in the early shrinkage stage.

Swelling of all layers between 0 and $100 \mathrm{~cm}$ was observed at 13 July and 18 July, caused by heavy rainfall in mid July, but hardly any swelling of the layer 0-150 cm was observed. The soil moisture content increased at 7.5, 22.5 and $45 \mathrm{~cm}$ depth, while soil moisture content at $80 \mathrm{~cm}$ was stable. Thickness change due to swelling and shrinkage below $50 \mathrm{~cm}$ depth was expected to be small, because the clay content is at most $5.9 \%$ (Tab. 4.1). The apparent shrinkage of the layer $0-150 \mathrm{~cm}$ might therefore originate from subsidence of the ground anchor in the (most probably) saturated zone at $150 \mathrm{~cm}$ depth. Shrinkage of the soil above $100 \mathrm{~cm}$ after 18 July, resulted in maximum total vertical shrinkage of $11.6 \mathrm{~mm}$ at 29 July, almost completely accounted for by the upper $60 \mathrm{~cm}$ of the soil.

The contribution of groundwater storage change to $\Delta W$ could not be considered for 2010, since groundwater level measurements only started at 22 July. Although, the soil moisture contents did exhibit an obvious response to groundwater level variations from August to November, the moisture content of the capillary fringe could not be estimated (Fig. 4.9C). The layer thickness changes and slowly rising groundwater level after substantial rainfall in early August showed that water was stored in the soil. From late August, when swelling was nearly complete and the soil was near saturation, groundwater level reacted rapidly to precipitation. The very slow recovery of layer thickness after late August corresponds to observations of swelling curves by Peng \& Horn (2007) of rapid swelling at first rewetting, followed by residual swelling at further wetting, when the moisture ratio increased but hardly any swelling was observed.

\subsubsection{Relation between volume change and soil water storage change in 2010}

The $\Delta V-\Delta W$ relationship obtained from measurement in 2010 and application of Eq. 4.4 (not accounting for $r_{\mathrm{s}}$ variations with soil moisture content and swelling or shrinking stage) is shown in Fig. 4.10. The precipitation and evapotranspiration dynamics caused variation in soil water storage change with respect to the starting date and accompanied alternating swelling and shrinkage periods. Water loss in the structural shrinkage phase could not objectively be distinguished, due to scatter in the relation between storage change and volume change, partly caused by the residual swelling occurring from September to November. Maximum soil volume was expected to occur in the structural shrinkage phase and a linear relation $(\Delta V=a \Delta W+b)$ was fitted through all datapoints with water storage smaller than water storage at maximum volume (Fig. 4.10). By applying this procedure, the number of points the fit was based on varies between layers and sensors. Fitting parameters and correlation coefficient $R^{2}$ are summarised in Tab. 4.3.

There was a mismatch between the response of water storage change based on EC-5s and volume change, at least at the measurement frequency and scale used here. For the 0-30 $\mathrm{cm}$ layer, swelling in the dry end of the curve for example was not 

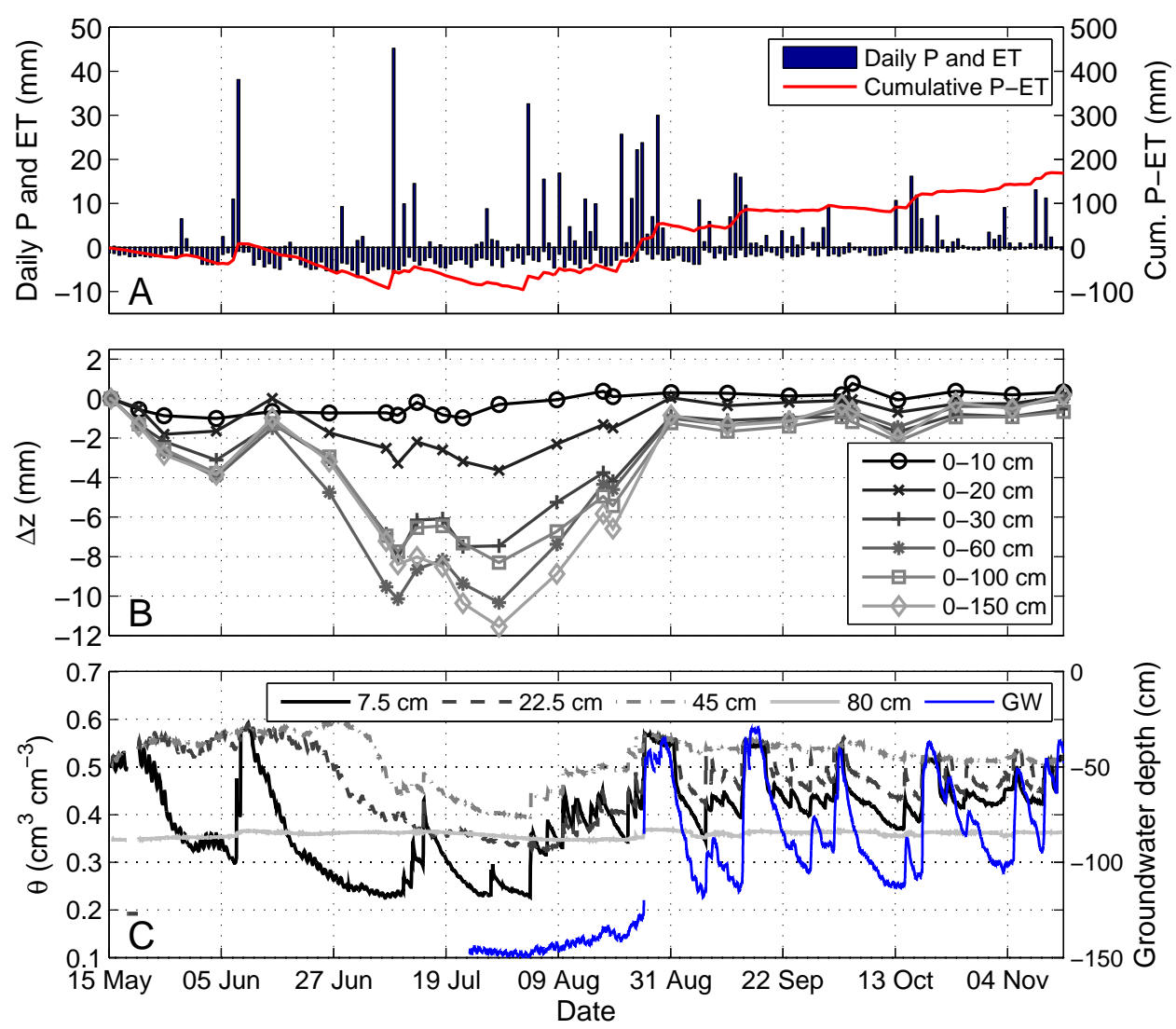

Fig. 4.9 Daily precipitation $P$, daily evapotranspiration $E T$ and cumulative net precipitation $P$-ET (A), cumulative layer thickness change $\Delta z$ in six soil layers (B), volumetric soil moisture content $\theta$ from CS616 sensors at four depths and groundwater depth (C) at field C from 15 May until 15 November 2010. N.B. Soil moisture data logging failed between 18 May and 20 May, groundwater level measurements started at 22 July.

Table 4.3 Fitted parameters for cumulative volume change outside the structural shrinkage phase, where $\Delta V=a \Delta W+b$, and correlation coefficient $R^{2}$ between fit and observations, for 2010 .

\begin{tabular}{ccccc}
\hline Sensor & Layer $(\mathrm{cm})$ & $a$ & $b$ & $R^{2}$ \\
\hline \multirow{5}{*}{ EC-5 } & $0-10$ & 0.21 & 0.28 & 0.45 \\
& $0-20$ & 0.21 & -2.29 & 0.90 \\
& $0-30$ & 0.38 & -5.93 & 0.81 \\
& $0-60$ & 0.38 & -9.61 & 0.88 \\
& $0-100$ & 0.21 & -9.76 & 0.71 \\
CS616 & $0-150$ & 0.21 & -13.37 & 0.92 \\
\hline \multirow{5}{*}{} & $0-10$ & 0.22 & 1.67 & 0.55 \\
& $0-20$ & 0.24 & 1.91 & 0.90 \\
& $0-30$ & 0.36 & 1.75 & 0.90 \\
& $0-60$ & 0.33 & -0.65 & 0.88 \\
& $0-100$ & 0.24 & -3.62 & 0.90 \\
& $0-150$ & 0.33 & -3.90 & 0.94 \\
\hline
\end{tabular}



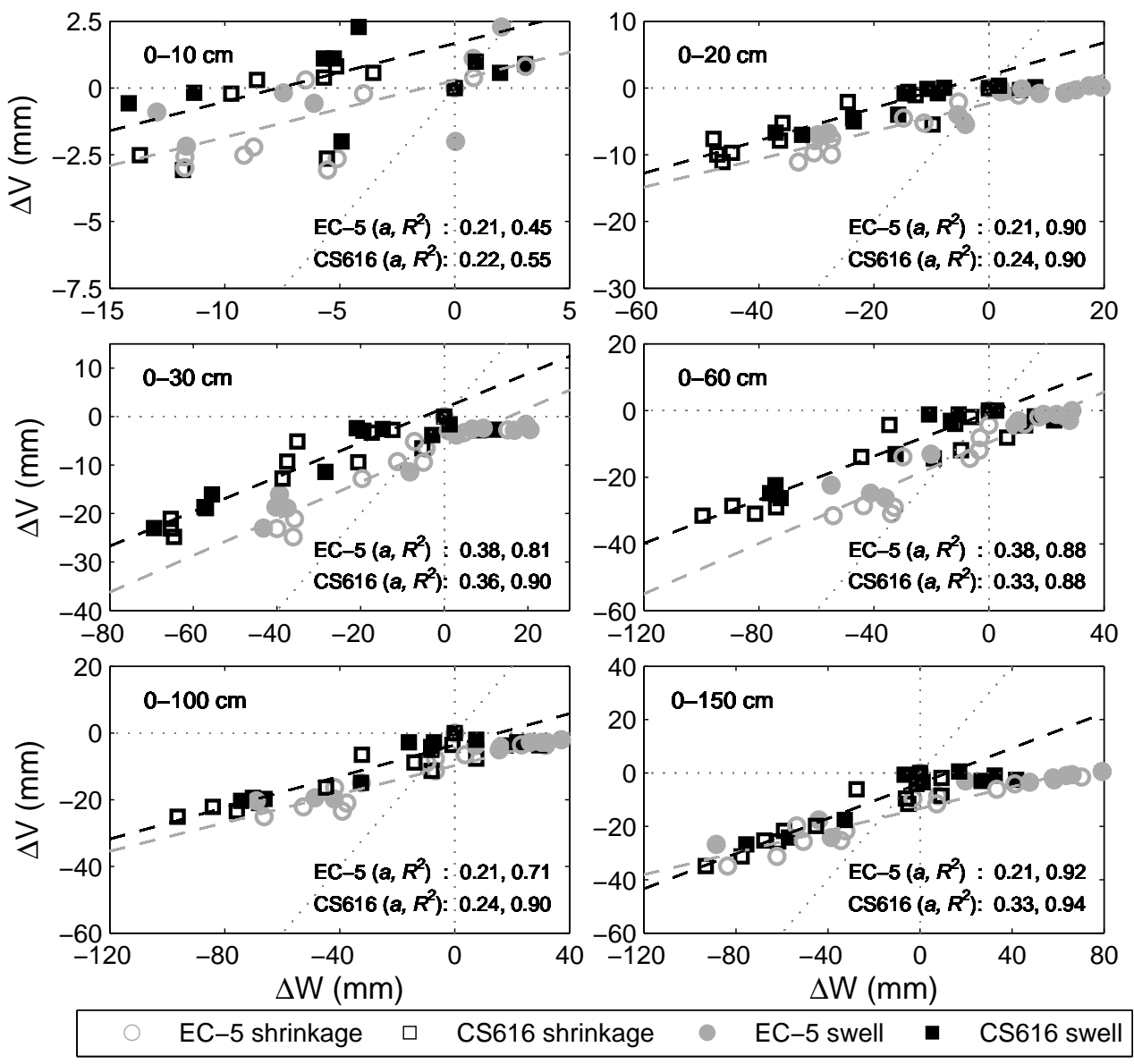

Fig. 4.10 Relationship between volume change per unit area $\Delta V$ and water storage change $\Delta W$ (EC-5 and CS616) at field C in 2010, for six soil layers. Dashed lines represent linear regression fits through data points outside the structural shrinkage phase, with $a$ indicating the slope of the fit and $R^{2}$ being the correlation coefficient. N.B Note the difference between the X and Y-axes.

accompanied by the expected increase in water storage (Fig. 4.10). Hysteresis between swelling and shrinkage was also observed by Peng \& Horn (2007). Since individual swelling stages, except for the final swelling stage, did not restore the soil volume to its maximum volume and were not accompanied by expected water storage increase, net $\Delta W$ was larger than net $\Delta V$, resulting in mild slopes. The effect of variable conditions, with alternation of shrinkage and swelling was enhanced by a difference in measurement scale of soil surface elevation change by ground anchors and soil moisture content by EC-5 sensors. Soil water content and volume change mainly occurred in parts of the soil that were in close contact with the atmosphere (e.g. the top of the soil and inter-aggregate pores), and less from the interior of aggregates in which the EC-5s were measuring. The small measurement volumes of EC-5s were thus relatively shielded from water content changes, and not representative for the profile-scale at 
which the volume change measurements were acquired. The measurement scale of the CS616s (including aggregates and inter-aggregate spaces) matched better with the scale of volume change measurements, resulting in less scatter for soil water storage change based on CS616 measurements, slightly higher $R^{2}$ values (Tab. 4.3), and no hysteresis in the dry range. For both sensor types $R^{2}$ for the linear fits were high, indicating a large range of basic shrinkage over the entire moisture range observed in 2010 .

Soil water storage changes calculated from the CS616s were larger than those calculated from EC-5s, mainly due to water loss at limited volume change. This again suggests that water loss in the structural shrinkage phase is larger in the bulk soil scale of CS616s than in the aggregate scale of EC-5s. As a result, fitted slopes of the $\Delta V-\Delta W$ relationship again were comparable for the two sensor types, except for the layer 0-150 cm (which will be discussed below).

Slopes varied with depth. In the upper layers slopes were low, probably because the effect of alternating occurrence of swelling and shrinkage was largest here. This effect was less in deeper layers and the largest slopes for both soil moisture sensors were observed in the layers $0-30$ and $0-60 \mathrm{~cm}$. In the sandy layers below $50 \mathrm{~cm}$, little volume change occured upon water storage change, resulting in small slopes in the layers $0-100$ and $0-150 \mathrm{~cm}$.

The clay content in the upper $50 \mathrm{~cm}$ was comparable to field B, but slopes were much lower, as compared to both Fig. 4.5 and Fig. 4.7. If $r_{\mathrm{s}}$ variations with moisture content would have been considered, the slopes in Fig. 4.10 would probably have been even lower, but applying swelling and shrinkage $r_{\mathrm{s}}$ values would be highly subjective as appropriate values are not available in literature. The mild slopes can be regarded as the net result of no shrinkage of rigid soil particles and reduced (residual or zero) shrinkage of dry surface layers of aggregates combined with normal shrinkage in the clay aggregates interiors (Yule \& Ritchie, 1980a,b). The magnitude of maximum vertical shrinkage at field B in 2010 was comparable to field C (data not shown), but the $\Delta V-\Delta W$ relationship could not be considered due to soil moisture measurements failures. The comparable clay content in both fields and the comparable observed vertical shrinkage at both fields in 2010 lead to the conclusion that the variation in shrinkage stages within a soil layer was caused and/or enhanced by the changes in weather forcing (precipitation, temperature, solar radiation) as observed in 2010.

The relatively large slope for the 0-150 cm layer based on CS616 measurements compared to EC-5 measurements is striking. Since no groundwater storage change estimation could be made, water storage change in this layer was largely determined by moisture content changes in the CS616 sensor installed at $80 \mathrm{~cm}$ depth. Moisture contents measured by the EC-5 and CS616 at this depth varied only slightly in the dry periods. The EC-5 at $100 \mathrm{~cm}$ however, recorded a considerable moisture content decrease (data not shown), resulting in the relatively large water storage change based on EC-5 measurements in the 0-150 cm layer. The CS616 sensors may have underestimated the total water storage decrease in the $0-150 \mathrm{~cm}$ layer due to limited coverage by only four sensors, thereby overestimating the slope in the $\Delta V-\Delta W$ relationship. This indicates the need for inclusion of data on groundwater and capillary fringe depth, for soil water storage change calculations. Preferred root water uptake 
by sugar beets from deep layers, as reported by Brown et al. (1987) and Camposeo \& Rubino (2003) might be an explanation for water content changes at $100 \mathrm{~cm}$ depth.

\subsection{Conclusions and outlook}

Basic, linear, shrinkage is the major shrinkage phase in a clayey field soil under continuous drying, on both aggregate scale and bulk soil scale, including cracks, structural pores, and multiple aggregates. Linear shrinkage with much smaller slopes was observed in the case of a more dynamical drying regime, with variation of dry and wet periods. Bronswijk \& Evers-Vermeer (1990) concluded that clay soils under Dutch climatic conditions experience a large degree of normal shrinkage, based on aggregate void and moisture ratios at pressure heads from 0 to $-16000 \mathrm{~cm}$. Based on observations presented here we can confirm this conclusion for field soils, although slopes of the $\Delta V-\Delta W$ relationship are much smaller than observed by Bronswijk \& Evers-Vermeer. Shrinkage slopes larger than unity were observed in clayey field soils experiencing a large range of soil moisture contents, when applying the isotropic shrinkage approach from Bronswijk $(1990,1991 \mathrm{~b}, \mathrm{a})$. We found clear evidence that the shrinkage geometry varied with moisture content.

We fitted a realistic range of shrinkage geometry factors to the observed soil moisture content range and assessed the difference in $\Delta V$ for the unsaturated zone when applying $r_{\mathrm{s}}(\theta)$ values and $r_{\mathrm{s}}=3$ to observed vertical shrinkage. For the $r_{\mathrm{s}}(\theta)$ range used here, we found an overestimation of $\Delta V$ up to $26.4 \%$ (17.5 mm) for the actively shrinking soil layer between surface level and $60 \mathrm{~cm}$ depth, when assuming constant isotropic shrinkage.

At the bulk soil scale, the structural shrinkage phase accounts for a large share of water loss of the profile. Upper layers experience structural shrinkage at the beginning of drying, but lower parts remain wet for a long time under influence of the ground water level and the capillary fringe. At delayed drying, these lower parts will also experience structural shrinkage, while the drier upper soil experiences normal, residual or zero shrinkage, causing the net shrinkage of the entire soil profile to be less than normal. At the end of the measurement period, water loss in the structural shrinkage phase was approx. $43 \%$ of total water loss for the 0-60 cm layer, and up to approx. $62 \%$ for the $0-150 \mathrm{~cm}$ layer. The remainder of water loss occurred in the basic shrinkage phase. Textural layering, soil moisture content gradients, and groundwater level are therefore important factors determining total soil-profile volume change and its relation with soil water storage change.

Under variable weather (many alternating wet and dry periods), both hysteresis in swelling and shrinkage and the different shrinkage phases represented in the profile simultaneously, further decrease the slopes of field shrinkage curves. Nevertheless, the $\Delta V-\Delta W$ relationship remains linear. Soil moisture measurements in soil volumes spanning multiple aggregates and cracks remain robust under these circumstances.

In order to use soil surface elevations changes as a proxy for soil water storage change in clayey soils, $r_{\mathrm{s}}$ variations with soil moisture, the effect of variable drying and wetting conditions, and the water loss in the structural shrinkage phase have to be considered, since solely relying on surface level elevation changes will lead to 
a large underestimation of soil water storage variations. In different wetting/drying regimes, the effective $r_{\mathrm{s}}-\theta$ relation calculated here will be affected by hysteresis between swelling and shrinkage. The effects of both processes on $\Delta V$ calculations are assessed separately in this study. In an alternating wetting and drying regime both processes are expected to be present, and the effects on $\Delta V$ calculations need to be assessed simultaneously by independent measurements.

Current and future technologies like GPS, satellite or airborne radar interferometry (InSAR), and airborne laser scanning (LIDAR) may be capable of measuring elevation changes with sufficient vertical and temporal detail on larger (field to catchment) scales (Gabriel et al., 1989; Bamler \& Hartl, 1998; Gao, 2007). However, more detailed shrinkage relations have to be applied to estimate storage changes in soil profiles from these measurements. Since the slope of the relationship between $\Delta W$ and $\Delta V$ depends on the drying regime and measurement scale, direct translation of shrinkage curves obtained through conventional laboratory tests on clay aggregates needs to be applied with care. The progress in theoretical conversions from laboratory shrinkage curves to field curves over the past years (e.g. Chertkov, 2005, 2012a; Stewart et al., 2016) might provide a solution to this issue in the future. 


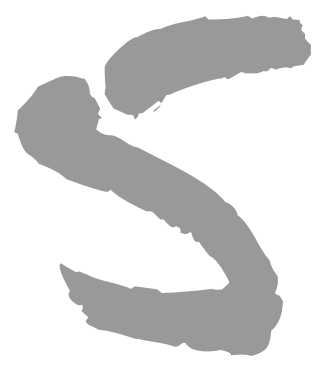

\section{Satellite based radar interferometry to estimate large-scale soil water depletion from clay shrinkage: possibilities and limitations}

\subsection{Introduction}

The need for large scale measurements and the ongoing improvements in remote sensing techniques and data processing tools, have considerably increased the value of remote sensing for hydrological and vadose zone studies in the last decades. Techniques can provide qualitative or quantitative information on the spatial distribution and temporal dynamics of parameters or variables of interest. Remote sensing therefore contributes to solving scaling issues in vadose zone hydrology, either in addition to traditional methods or as stand-alone measurement techniques. With current knowledge and the (future) availability of improved systems, techniques, and models, the value of remote sensing products is expected to increase further. This creates opportunities for new applications, which might have been considered not feasible before. It is worth investigating the potential, limitations and controlling factors for these new applications, and to consider these in the design of new remote sensing platforms and missions. The potential and applications of many ground-based, airborne and spaceborne remote sensing techniques for hydrological, hydrometeorological and

\footnotetext{
This chapter is a modified version of: Te Brake, B., Hanssen, R.F., Van der Ploeg, M.J., and De Rooij, G.H.: Satellite-based radar interferometry to estimate large-scale soil water depletion from clay shrinkage: possibilities and limitations. Vadose Zone J., vol. 12, num. 3, 2013. doi:10.2136/vzj2012.0098-177.
} 
vadose zone studies have received ample attention in the last decades (e.g. Ulaby et al., 1996; Pietroniro \& Leconte, 2000; Jackson, 2002; Huisman et al., 2003; Wagner \& Pathe, 2005; Wagner et al., 2007, 2009; Bronstert et al., 2012). Studies to the application of Interferometric Synthetic Aperture Radar (InSAR) are relatively limited. InSAR is a remote sensing method capable of mapping very small surface deformations, using the phase information of two Synthetic Aperture Radar(SAR) images taken of the same area on different times. Hydrological applications of InSAR are largely restricted to hydrogeological studies (Smith, 2002). Galloway et al. (1998) used InSAR to map land subsidence caused by aquifer compaction in the Antelope Valley, Mojave Desert, CA, and estimated aquifer parameters to simulate non-elastic aquifer compaction. Amelung et al. (1999) derived surface deformations from InSAR in Las Vegas, NV, to determine aquifer and aquitard characteristics. Reeves et al. (2011) linked InSAR data to elastic aquifer deformation in an agricultural area in the San Luis Valley, CO, using high quality subsets of InSAR data. Calculated temporal change of storage coefficients and hydraulic heads showed the potential of current InSAR data and developing processing techniques, but errors in hydraulic head measurements were still unacceptably large for aquifer modeling. Further improvements of this topic in the same area, promising for operational use, were later developed by Reeves et al. (2014) and Chen et al. (2016). InSAR applications to surface water hydrology include attempts to map water level changes in lakes and wetlands (Alsdorf et al., 2001; Wdowinski et al., 2004; Lu \& Kwoun, 2008; Hong et al., 2010).

InSAR studies of links between surface deformations and vadose zone processes are scarce up to now. Some InSAR studies have attributed observed phase changes over agricultural fields to soil moisture variations (Gabriel et al., 1989; Hanssen, 2001; Van der Kooij et al., 1995; Gens \& Van Genderen, 1996; Massonnet et al., 1997; Nolan et al., 2003), but often such observations remained qualitative and were regarded as unrelated to the objectives of the study. Gabriel et al. (1989) used records of applied irrigation onto 52 agricultural fields to explain observed differential phase changes and found a match for 48 fields. A validation based on dedicated field measurements was not performed, since the objective of their study was not to quantify soil water storage changes. Nolan \& Fatland (2003) argued that two different sources of a soil moisture phase change exist: (i) phase change due to changes of dielectric properties and penetration depth, and (ii) phase change due to changes in soil surface elevation level (geometric effects). Their results confirmed earlier laboratory experiments by Rudant et al. (1996), who reported ambiguous effects of dielectric and geometric effects of soil moisture. Other modeling and laboratory studies to the relation between interferometric phase change and soil moisture content have been carried out by Nesti et al. (1998), Ben Khadhra et al. (2006), Rabus et al. (2010), Morrison et al. (2011) and De Zan et al. (2014).

To our knowledge no studies have been conducted to quantify soil surface elevation changes from swelling and shrinkage of clays through InSAR and in situ measurements simultaneously. Potentially, InSAR can be used to measure soil elevation changes at high spatial and temporal resolution on small catchment to field scale. In areas with clayey soils these elevation changes can be qualitatively linked to soil water storage changes as has been shown repeatedly (Yule \& Ritchie, 1980a,b; Bronswijk, 
1991b; Cabidoche \& Ozier-Lafontaine, 1995; Cabidoche \& Voltz, 1995; Kirby et al., 2003, and Ch. 4). Linking InSAR to swelling and shrinking of clayey soils, may improve quantification of soil water storage at scales relevant for current hydrological models, water management, and policy making. These improvements are needed, as hydrological models still produce highly uncertain representations of storage terms (Creutzfeldt et al., 2010).

In this chapter InSAR principles are introduced with a focus on hydrological applications. Limitations and potential applications of surface elevation changes related to unsaturated zone processes will be explained and demonstrated. A comparison between in situ measurements and data from the TerraSAR-X and TanDEM-X ${ }^{1}$ satellites in an agricultural area with clayey soils will be made.

\subsection{Satellite based radar interferometry (InSAR)}

After the introduction of InSAR in Ch. 2, this section will discuss InSAR principles more specifically for unsaturated zone hydrology applications, with an emphasis on swell and shrinkage measurements of clayey soils.

\subsubsection{InSAR measurements of deformation}

The interferometric combination of two Synthetic Aperture Radar (SAR) images acquired at different times, as done in repeat-pass interferometry, yields the interferometric phase $\varphi$. The interferometric phase depends on differences in path length (range) and propagation velocity between the two acquisition times and geometries. In general terms:

$$
\varphi=-2 \pi a+\varphi_{\text {flat }}+\varphi_{\text {topo }}+\varphi_{\text {defo }}+\varphi_{\text {atmo }}+\varphi_{\text {noise }},
$$

where $a$ is the integer phase ambiguity number, i.e. the number of full phase cycles and $\varphi_{\text {flat }}, \varphi_{\text {topo }}, \varphi_{\text {defo }}, \varphi_{\text {atmo }}$, and $\varphi_{\text {noise }}$ are the flat Earth phase, topographic phase, deformation phase, atmospheric phase, and phase noise (see Ch. 2).

For unsaturated zone applications, an additional term for phase changes from changes in dielectric properties of the soil (De Zan et al., 2014) might need to be included, but in this study phase change resulting from deformation is the most important term. Deformation-induced phase change is related to surface motion $\Delta z$ in the radar line of sight (LOS) through the radar wavelength $\lambda$ :

$$
\varphi_{\text {defo }}=\frac{4 \pi}{\lambda} \Delta z \text {. }
$$

This relationship is only valid if the scattering contribution to interferometric phase is similar in both images and the imaging is coherent. If this condition is met, the relative line of sight (LOS) movement of scatterers with respect to a reference location in the image can be measured as a fraction of the wavelength, yielding millimetre to centimetre accuracies for X-, C-, and L-band SAR (Hanssen, 2001).

\footnotetext{
${ }^{1}$ As TerraSAR-X and its 'twin brother' TanDEM-X are identical, and individually or combined suitable for interferometry, we will further refer to the SAR imagery as TerraSAR-X (or TSX) data.
} 


\subsubsection{Interferometric characteristics and limitations}

\section{Topographic and atmospheric phase}

To obtain a measurement of deformation (the variable of interest in this study), all other factors influencing the interferometric phase should be eliminated. The sensitivity of phase change to topography, is much lower than its sensitivity to deformation, but combining two acquisitions with a short relative distance between the orbits of the two satellite passes (short perpendicular baseline) enables the construction of accurate digital elevation models (DEMs) (e.g. Zebker \& Goldstein, 1986). Based on this, Gabriel et al. (1989) introduced a method to eliminate topographic phase in an interferogram, using the topographic phase contribution calculated from an interferometric combination of one of the images with a third image under the assumption of zero deformation. Using this three pass-method of differential interferometry (DInSAR) they revealed very small surface motions over agricultural fields at Imperial Valley, CA. A similar method to eliminate topographic phase can be used if an accurate external DEM is available. Methods to eliminate atmospheric contributions from single interferograms are less straightforward (Hanssen \& Klees, 1999; Foster et al., 2006). Multi-interferogram techniques might be required to mitigate atmospheric artefacts from to the interferometric phases.

\section{Decorrelation}

Interferometric processing can only deliver useful information if scattering characteristics are nearly equal during the acquisitions of master and slave image (coherent scattering). The main factors responsible for decorrelation of the received signals are changes in geometric configuration (geometric decorrelation) and temporal changes in scattering characteristics (temporal decorrelation). Geometric decorrelation results from slightly different viewing angles in two images, causing the contributions from scatterers within a resolution cell to add up differently. Large perpendicular baselines therefore lead to decreased correlation (Gatelli et al., 1994) and selecting image pairs with short perpendicular baselines is preferable, provided that their temporal baseline (the time between the acquisitions) is small enough to minimize temporal decorrelation. Any change in scattering of the surface between master and slave acquisition (due to vegetation, snow, soil tillage etc.) may lead to temporal decorrelation (Zebker \& Villasenor, 1992). Also the wavelength of the radar, relative to the size of scatterers, affects decorrelation: if the wavelength is relatively small, the radar signal will not penetrate through vegetation and scattering will be highly random. Studying dynamic (unsaturated zone) processes requires short temporal baselines, both to capture the dynamics and to minimize decorrelation over vegetated surfaces. A decreased sensitivity to vegetation cover can be accomplished by using long wavelength SAR systems, but for studying small deformation rates, the phase needs to be highly sensitive to deformations, which is in turn provided by short wavelengths. Recent (ALOS: L-band, ENVISAT and ERS: C-band, with 46, 35, and 35 days repeat intervals) and current (COSMO-SkyMed, TerraSAR-X/TanDEM-X: X-band, and RADARSAT-2 and Sentinel-1: C-band, with 8, 11, 24, and 6 days repeat interval) SAR satellite systems in orbit, all have different characteristics in terms of wavelength and repeat cycle. 
Data selection will therefore always involve a trade-off between the spatial baseline, the temporal baseline and the sensors wavelength. In this chapter, we will consider implications of temporal baselines using TerraSAR-X data over an agricultural area in the Netherlands.

\section{Phase unwrapping}

Because of the cyclic nature of the relative phase, measurements are only possible in the oscillation range between $-\pi$ and $\pi$. This is called the wrapped phase. Wrapped phase cycles (also called fringes in interferograms), occur in an interferogram if relative phases change gradually and consistent over an area that gives a coherent signal. The procedure to estimate the absolute phase value from the relative phase gradient is called phase unwrapping. In the case of clear phase fringes, unwrapping is relatively straight forward, because spatial patterns allow interpretation of the magnitude and direction of deformation or topography, although assumptions have to be made on the gradient of the absolute phase (e.g. Goldstein et al., 1988). If high degrees of decorrelation occur, or phase change is discontinue (e.g. bound by isolated structures, such as individual agricultural fields or buildings), unwrapping is challenging (Goldstein et al., 1988).

\subsection{Materials and methods}

\subsubsection{In situ measurements}

To relate in situ water storage changes to InSAR derived deformations, a field campaign was carried out from April 2010 to October 2011, in the Purmer area, approximately $15 \mathrm{~km}$ north of Amsterdam, Noord-Holland, the Netherlands. The Purmer area is a polder with clay holding soils of marine origin, with agriculture (grassland and crop rotation of potatoes, maize, sugar beets, and wheat) being the most prominent land use type. TerraSAR-X data were acquired over the study area in the same period as the field measurements were conducted. We focus on data from 2011 of two adjacent agricultural fields (Fig. 5.1), cropped with winter wheat (field A) and Kentucky Bluegrass for seed harvesting (field B). Ground anchors were installed at various depths that allowed precise monitoring of the vertical layer thickness changes between anchoring depth and soil surface (Bronswijk, 1991b, Ch. 4). At the ground anchors location, mean clay content of the upper $60 \mathrm{~cm}$ of the soil was $19.3 \%$ (18.0$23.6 \%$, field A) and $24.9 \%(24.4-25.3 \%$, field B), but variation in clay content has been observed across both fields (see also clay fractions reported in Ch. 3 ). The clay fraction mainly consisted of montmorillonite minerals.

Ground anchors were installed at various depths that allowed precise monitoring of the vertical layer thickness changes between anchoring depth and soil surface (Bronswijk, 1991b, Ch. 4). The layer thickness changes of the unsaturated zone are the primary observation of interest to link vertical deformations to soil water storage changes. Soil water storage changes were calculated from data from EC-5 capacitance sensors (Decagon Devices, 2010) and CS616 water content reflectometers (Campbell Scientific, 2006). Rainfall rates were measured by a Parsivel disdrometer (OTT Hydrometry Ltd) located approximately $150 \mathrm{~m}$ from the measurement location at field A. Daily 


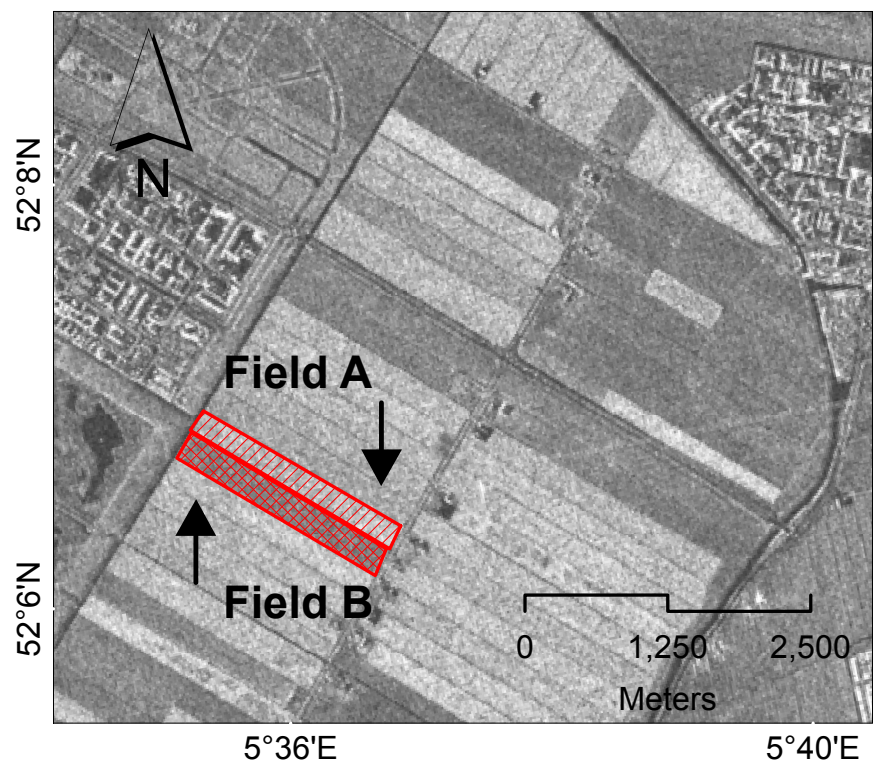

Fig. 5.1 Interferometric magnitude image of the northern part of the Purmer area showing the location of field A and field B.

values of reference potential evapotranspiration and hourly air temperature data were obtained from the Royal Netherlands Meteorological Institute (KNMI, 2014) weather station in Berkhout (ca. $16 \mathrm{~km}$ north of the field site). Precipitation and evapotranspiration data are only used here for a first indication of hydrological status of the soil and not for quantification of soil water storage change in terms of water balance purposes. Therefore no estimation of actual evapotranspiration was made and we refer to the reference potential evapotranspiration as evapotranspiration in the remainder of this chapter. in situ measurements were performed on the day of satellite pass, and occasionally one day later. The measurement campaign, the data it generated, and the data processing and analysis are discussed in detail in Ch. 4.

\subsubsection{TerraSAR-X data}

We processed interferograms from TerraSAR-X data over the province of NoordHolland, north of Amsterdam, the Netherlands. A large part of the area consists of agricultural areas with clayey soils (see Fig. 5.4). Several reclaimed polders (among which the Purmer area) are situated in the scene. Data from the ascending orbit in HH-polarization were used. The TerraSAR-X SAR instrument operates at a wavelength of $31 \mathrm{~mm}$ (frequency of $9.65 \mathrm{GHz}$ ), with a repeat cycle of 11 days. Spatial resolution of the data was approximately $3 \mathrm{~m}$. Image acquisition time over NoordHolland was 17:18 UTC for all images. 


\subsubsection{Information content of phase and in situ observations}

An InSAR phase measurement is a temporal interferometric difference between the acquisition time of the master and slave image, for a resolution cell $i$. However, there is one unknown (random) phase bias that is affecting all pixels equally. Spatial differences between pixels eliminate this bias. Hence, the first interpretable InSAR observation is the double-difference, in general terms noted as $\mathrm{d}_{r i}^{m s}$. The observation is a difference both temporally (between master $m$ and slave $s$ acquisition time) and spatially (with respect to some reference pixel $r$ ) (Marinkovic et al., 2008; Van Leijen et al., 2005). To resolve the incompatibility in information content between InSAR (double difference) and in situ (single difference) measurements, either the InSAR observations or the in situ measurements have to be converted. When measuring elevation change or soil layer thickness change $\Delta z$ resulting from swell and shrinkage of clays, measurements of temporal changes are obtained (e.g. at master $m$ and slave $s$ acquisition time) for a fixed point. For a measurement location $\mathrm{A}$ this can be notated as $\mathrm{z}_{A}^{m s}$ and the double difference layer thickness change between master and slave acquisition time and between measurement locations $\mathrm{A}$ and $\mathrm{B}$ can be calculated by:

$$
\Delta z_{A B}^{m s}=\Delta z_{A}^{m s}-\Delta z_{B}^{m s} .
$$

By relating InSAR double differences to a non-moving target, they can be converted to single differences. This has not been applied in this study, but will be discussed in Ch. 6.

\subsection{Results}

\subsubsection{In situ measurements of clay shrinkage}

Figure 5.2 shows a clear relation between the precipitation deficit and vertical soil shrinkage in the top $100 \mathrm{~cm}$ of field A and B between 17 March 2011 and 3 June 2011; a predominantly dry period interspersed with some small rain showers. After 19 April, shrinkage in field A exceeded that of field B, reflecting the increased water uptake by the winter wheat, which entered a growth phase around that time, and despite the higher clay content at Field B.

By assuming the shrinkage to be isotropic (Bronswijk, 1990, 1991b) and relating the shrinkage to the observed changes in soil water content, we derived an in situ soil shrinkage curve (Fig. 5.3). This curves resembles shrinkage curves obtained in the laboratory for individual clay clods (e.g. Ross \& Prebble, 1989; Bronswijk, 1990), displaying a large range of linear shrinkage. Only thickness changes of $100 \mathrm{~cm}$ deep layers are considered here, since no additional shrinkage occurred between $100 \mathrm{~cm}$ depth and the groundwater table which gradually declined from 132 to $150 \mathrm{~cm}$ below surface level in the period considered here. The assumption of constant isotropic shrinkage used here is a rough simplification, as discussed in Ch. 4. However, the strong relation between soil water storage and volume change illustrates the potential of surface elevation change measurements to quantify soil water storage change. 


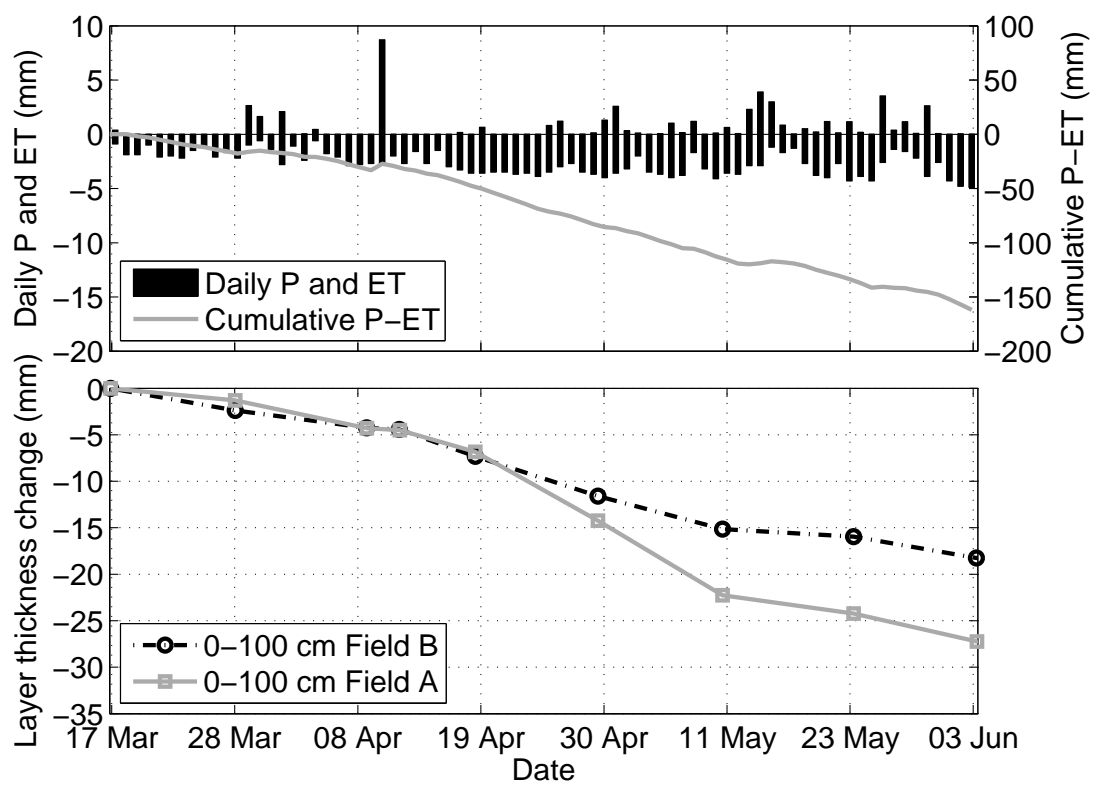

Fig. 5.2 Daily precipitation $\mathrm{P}$, daily evapotranspiration $E T$ and cumulative net precipitation $P$ ET (top) and cumulative layer thickness change with respect to the starting date at field A and B (bottom) from 17 Mar. 2011 until 3 June 2011.

\subsubsection{InSAR observations related to land use, soil type and frost heave}

Figure 5.4A and 5.4B display interferometric magnitude and phase from TerraSAR-X data over Noord-Holland, including polders Schermer (S), Beemster (B), Purmer (P), and Wieringermeer (W). Master and slave acquisition dates were 12 February 2011 and 23 February 2011. Because the polders are topographically about 5 meter lower than their surroundings (Fig. 5.4E), the topographic phase in all interferograms in this chapter was removed using an external DEM from the Shuttle Radar Topography Mission (SRTM).

The interferogram is highly coherent, especially in the polders as seen from clear phase changes bounded by the boundaries of individual agricultural fields and other surface features. Phase changes between adjacent fields are abundant and very clear and extensive checkerboard patterns generated by the layout of the parcels, as was also observed by Gabriel et al. (1989) and Van der Kooij et al. (1995). Outside the polders, the interferometric magnitude is generally less, and agricultural fields are less clearly distinguished based on phase and coherence. The simplified land use map in Fig. 5.4C (derived from Dutch land use map LGN-5 (Hazeu, 2005)), is based on older data than those coming from the SAR acquisitions, but the division between grassland and rotational crops is expected to be valid in all but few cases. Patterns in land use show a striking resemblance with the magnitude image; bright areas over urban areas and agricultural crops and darker areas over grassland, both in and outside the polders. Since the interferogram is constructed from two images from 


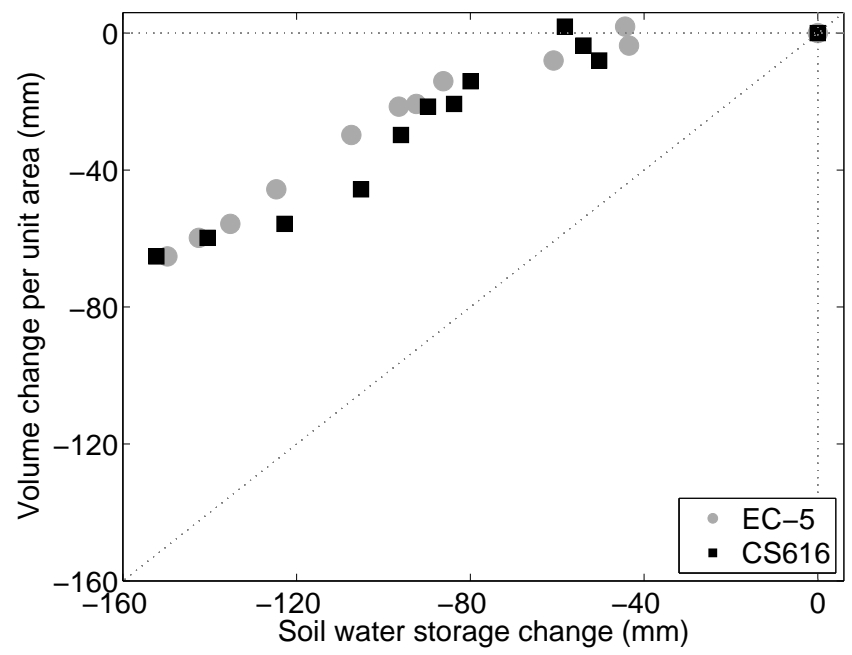

Fig. 5.3 Relation between volume change per unit area and water storage change from EC-5 and CS616 sensors between 0 and $100 \mathrm{~cm}$ depth at field B.

February 2011, the agricultural crop fields were mostly bare. Ploughing in this area is generally done in late autumn, allowing the clay-rich soil to be crumbled during frost periods. This resulted in a rough surface in February, causing higher backscatter intensity (and higher magnitude) than grassland.

Figure 5.4D shows a simplified soil map, based on a soil map provided by Alterra (2006). When comparing this soil map with the interferometric phase image of Fig. 5.4B, it can be seen that decorrelation of the interferometric phase over peat areas outside the polders is relatively high, and field boundaries cannot be clearly distinguished. Ditch density and ditch water level in peat areas is high to prevent peat oxidation. These wet conditions and the large area of open water in combination with the high spatial resolution of TerraSAR-X data, cause decorrelation of the signal.

Outside the peat areas, the interferogram displays clear phase differences between, urban areas, grassland and agricultural crop land, and phase jumps between adjacent agricultural crop fields. Van der Kooij et al. (1995) observed phase changes between agricultural fields in an ERS-1 (C-band) interferogram over Zeeland, an area with marine clay-rich soils in the Netherlands. Gens \& Van Genderen (1996) explained this phenomena to be related to soil surface heave due to freezing of water, with spatial variations in water content related to soil type causing differential phase change. This could be an explanation for the differential phase change between adjacent agricultural

Fig. 5.4 (next page) 11-day TerraSAR-X interferogram (magnitude (A) and phase (B)), land use (C), soil (D), and elevation maps (E) of Noord-Holland, with polders indicated by S, B, P, and W. The dashed line indicates the interferogram extent. Phase is wrapped between $-\pi$ and $\pi$. One color cycle is equivalent to $18 \mathrm{~mm}$ relative vertical displacement. The white square in E indicates the location of field A and B. (C and D: (C)Alterra; E: CAHN, (www.ahn.nl).) 

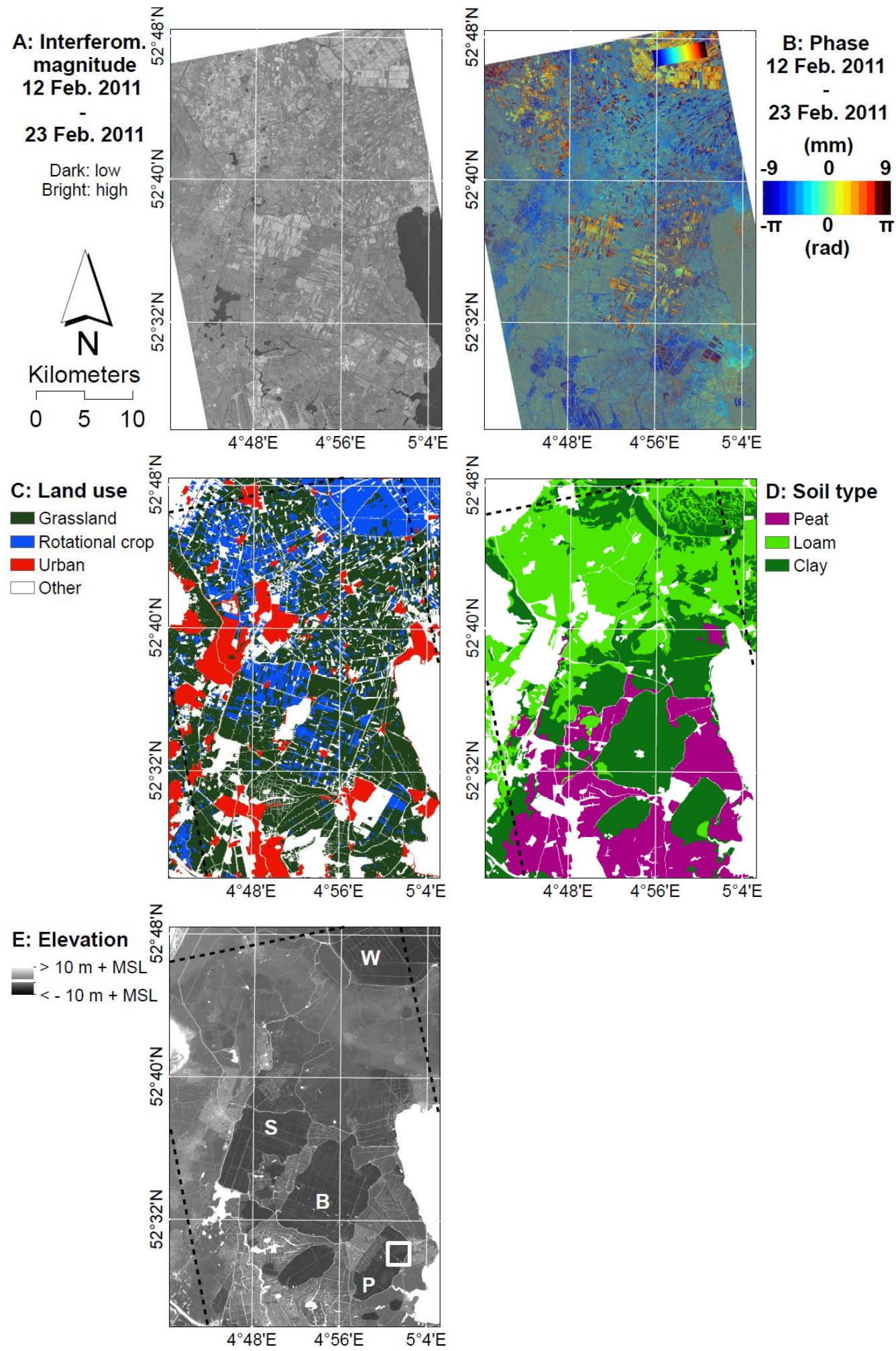
crop fields observed in Fig. 5.4B as well. Air temperatures (at $0.10 \mathrm{~m}$ above the ground) observed at the KNMI station in Berkhout (centrally located in the scene of Fig. 5.4) in the 48 hours prior to image acquisitions, were well above $0{ }^{\circ} \mathrm{C}$ (minimum $1.7{ }^{\circ} \mathrm{C}$ ) at 12 February, and well below $0{ }^{\circ} \mathrm{C}$ (maximum $-0.4{ }^{\circ} \mathrm{C}$ ) at 23 February. Therefore frost heave at the slave acquisition time is a probable explanation for the observed phase differences.

Additionally to phase change between agricultural crop fields, phase changes between land use types are observed in Fig. 5.4. Especially in the polders, differential phase changes occur between fields with grass and agricultural crops. Variations in soil moisture can occur between those fields induced by the difference in cover, but also grass can act as an insulator and reduce the flow of heat from the soil (Dagesse, 2010). This would cause a difference in soil temperature and therefore a difference in frost heave effects between agricultural crop fields that are bare in winter and grass land. An effect of freezing and thawing and soil moisture content variation was also suggested by Hanssen (2001) for phase changes between a ploughed field and a grass field in Groningen, the Netherlands.

Detailed phase analysis of three cascade processed interferograms (series of interferograms where the slave of an interferogram becomes the master of the next interferogram) using 4 acquisitions from 1 February 2011 to 6 March 2011, over field A and field B in the Purmer reveals similar results (Fig. 5.5). In computation of the interferograms, the oldest image of the interferometric pair was taken to be the master image. Wrapped phase and their equivalent 'apparent vertical motion' are indicated on the top and bottom x-axes in Fig. 5.5. Apparent vertical motion can be calculated from phase change using the imaging geometry with an incidence angle of $30^{\circ}$. Here we use the term 'apparent vertical motion', since phase changes can not be indisputably ascribed to deformation. Both phase change and apparent vertical motion are relative to a random and unknown point in the interferogram. Interpreting the phase values over the agricultural fields is therefore best done relative to each other as double differences.

The distributions of wrapped phases in the histograms have clear peaks at different phases for field A and field B, indicating a rather stationary phase changes within the individual agricultural fields. The lines coinciding with these peaks are the average phases, obtained by multilooking (averaging of the signal in the complex domain) over the entire individual agricultural fields. When considering mean phase change of field A with respect to mean phase change of field $\mathrm{B}$, a larger value for field A indicates a shift towards a slightly longer path of the microwave signal and therefore relative subsidence of field $\mathrm{A}$. This is the case in Fig. 5.5A and 5.5C, while relative uplift of field A with respect to field B is observed in Fig. 5.5B. The absolute phase difference in Fig. 5.5A is slightly larger than the absolute phase difference in Fig. 5.5B, but almost equal to the absolute phase difference in Fig. 5.5C. Air temperatures at 1 February (as low as $-6.2{ }^{\circ} \mathrm{C}$ before acquisition) and 23 February (see above) were sufficiently low to enable soil freezing, which was not the case at 12 February (see above) and 6 March (temperature at time of acquisition $2.7{ }^{\circ} \mathrm{C}$ and up to $5.3{ }^{\circ} \mathrm{C}$ in the hours before). Therefore it's probable frost heave occurred on 1 February and 23 February, and not on 12 February and 6 March. At master acquisition of 

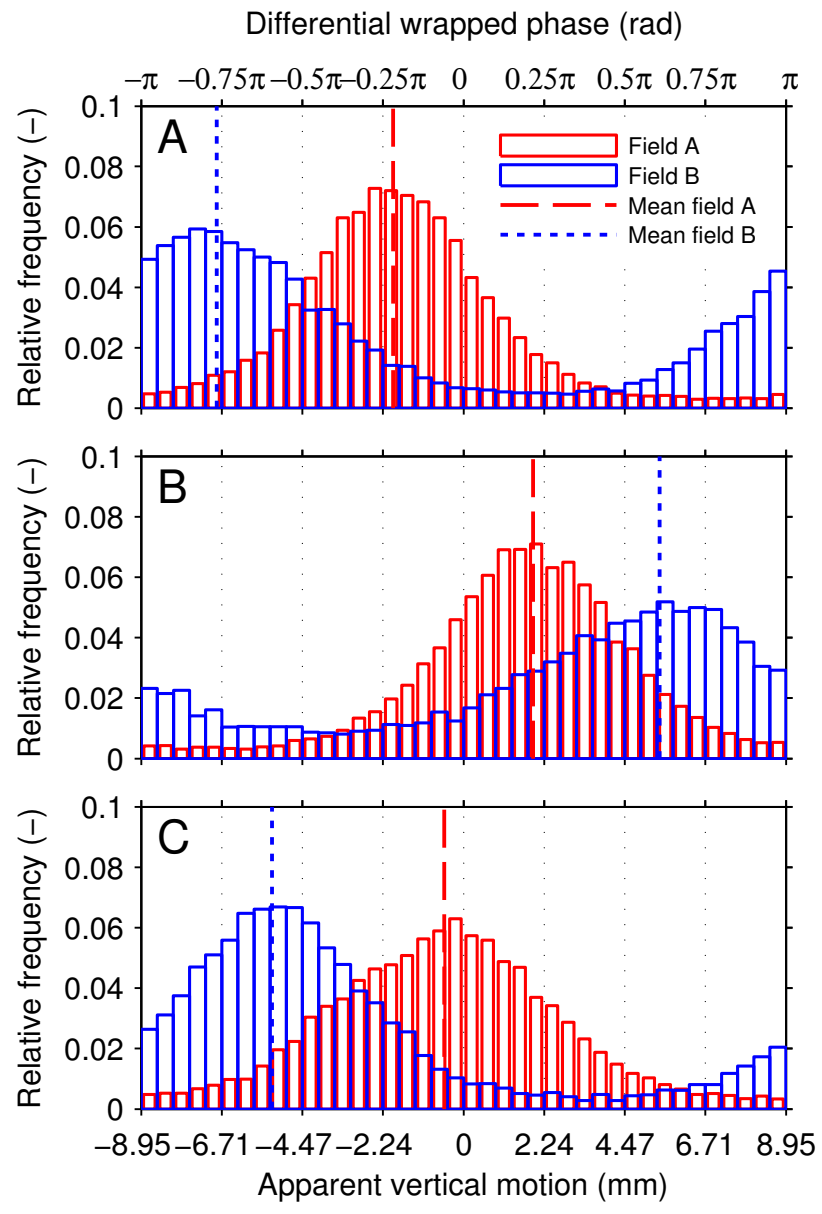

Fig. 5.5 Histograms of interferometric phase over field A and B showing differential phase change as a result of frost heave. A) Master: 1 Feb. 2011, slave: 12 Feb. 2011. B) Master: 12 Feb. 2011, slave: 23 Feb. 2011. C) Master: 23 Feb. 2011, slave: 6 Mar. 2011. Histograms show wrapped phase between $-\pi$ and $\pi$ on the top x-axes, and equivalent apparent vertical motion on the bottom x-axes. Dashed vertical lines indicate the mean phase values per field after multilooking over the entire agricultural field.

Fig. 5.5A and 5.5C, the soil at field A was frozen and elevated with respect to field B, where grass acted as an isolator, while at slave acquisition both soils were unfrozen. Freezing and thawing of the upper few centimetres of the soil, are known to be small for vegetated soil compared to bare soil with clay aggregates (Edwards \& Burney, 1989). No differential soil moisture content was observed.

In the interferogram of Fig. 5.5B, the soil at field A was frozen at slave acquisition. The relative motion appears in the opposite direction compared to Fig. $5.5 \mathrm{~A}$, because field A subsides with respect to field B upon thawing. Measurements with ground anchors on field $\mathrm{A}$ and $\mathrm{B}$ reveal the double difference surface elevation changes of field A with respect to field B of $3.49,-4.03$ and $2.61 \mathrm{~mm}$ at 12 February, 23 February 
and 7 March. The direction of motion from in situ measurements and InSAR is in good agreement, but magnitude of deformation differs slightly.

\subsubsection{Phase observations during the growing season}

Figure 5.6A shows interferometric phase for an 11 day interferogram (master: 26 June 2010; slave: 7 July 2010). Phase patterns originating from atmospheric signal delay are visible, recognizable as patterns not bound by surface features. These phase patterns need to be considered in phase interpretations and hamper phase analysis over large areas. Over many agricultural fields, the phase signal is decorrelated due to vegetation cover in the growing season, but still some fields can be clearly distinguished which can be selected for phase analysis. Opposite to the winter interferogram in Fig. 5.4B, the grassland areas in the polders often have higher coherence in Fig. 5.6A, and can therefore deliver more reliable phase estimates than the rotational crop fields. Vegetated surfaces (rotational crops) and forests show relatively high backscatter intensity (brighter colors in Fig. 5.4A), compared to grassland due to higher roughness and volume scattering of the microwave signal (Richards, 2009). The higher roughness and volume scattering do however not provide coherent phase estimates, because the scatterers will be unstable between the master and slave acquisition, as a result of vegetation growth, changes in the canopy structure, and wind effects on the crops orientation with respect to the satellite orbit. Grassland on peat areas have low coherence in both summer and winter, probably due to high water levels that are maintained to prevent peat oxidation. The presence of visually observable phase changes between adjacent fields, although sparse, indicates that even in the growing season areas can be identified to base phase analysis on. Atmospheric phase delay hampers the phase analysis considerably, but if phase patterns remain bound to ground features (like agricultural fields or buildings), phase changes over small distances could still be analysed, assuming uniform atmospheric effects over these features.

Figure 5.6B shows phase from an interferogram with a temporal baseline of 22 days due to a missing acquisition, with master and slave images of 19 April 2011 and 11 May 2011. Temporal decorrelation due to changes in scattering characteristics in a 22 day interval caused coherence loss over many agricultural fields (Fig. 5.6B). Generally, temporal decorrelation hampers high quality phase observations, especially in the onset of the growing season. This limits the possibilities to observe unsaturated zone processes, as these may act on short time-scales.

\subsubsection{Phase observations of clay shrinkage}

Figure 5.7 shows histograms of phase changes from cascade processed interferograms of TerraSAR-X acquisitions over field A and B in the Purmer area between 17 March 2011 and 2 June 2011, comparable to Fig. 5.5. Phase signals bounded by isolated structures like individual agricultural fields were observed, which leads to our assumption that atmospheric phase contribution can be neglected when comparing signals of two neighbouring fields. As the example in Fig. 5.6B shows, the length scale of atmospheric phase contributions is larger than the field scale. 

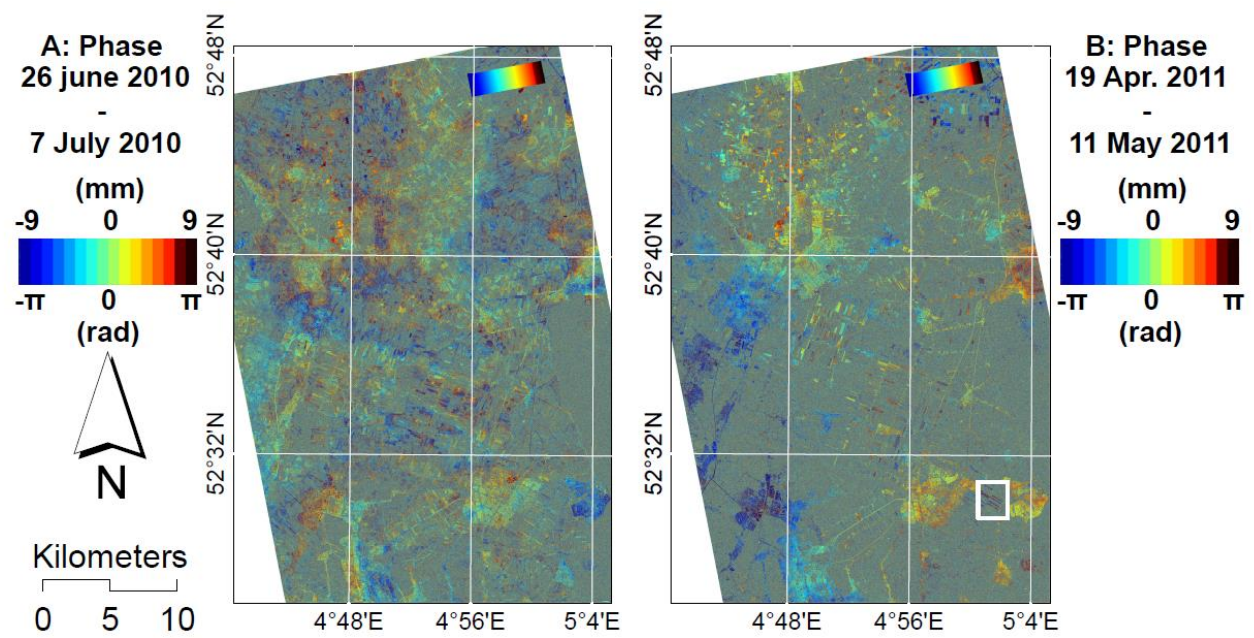

Fig. 5.6 TerraSAR-X interferograms over Noord-Holland showing decorrelation effects to phase changes. A) Phase images of an 11 day interferogram (master: 26 June 2010; slave: 7 July 2010). B) Phase images of a 22 day interferogram (master: 19 Apr. 2011; slave: 11 May 2011). Interferograms show wrapped phase between $-\pi$ and $\pi$. One color cycle is equivalent to $18 \mathrm{~mm}$ relative vertical displacement. The white square in B indicates the location of field A and B.

The histograms in Fig. 5.7A have clear peaks at certain phase values. The histograms become more uniform when noise levels increase. Noise levels do increase due to longer temporal baselines, as is the case in Fig. 5.7B and Fig. 5.7C, where interferograms were computed with 22 days temporal baselines because of missing acquisitions. Temporal decorrelation at the onset of the growing season between 19 April and 11 May further increases noise in Fig. 5.7C compared to Fig. 5.7B, especially in field $\mathrm{A}$ (winter wheat). The effect of vegetation cover is apparent in all later interferograms, but Fig. 5.7D and 5.7C are calculated from interferograms with temporal baselines of 11 days and consequently the spread in the histograms remains relatively small, despite crop growth in May. Phase distributions from later acquisitions in summer are generally more uniform and reliable phase estimates are hard to obtain.

Although less clear than in Fig. 5.5, all histograms in Fig. 5.7 show peaks to some extent. The mean values in the histograms are obtained by multilooking over the entire individual agricultural fields. Phase change between field A and B is generally small, except for the 22 day interferogram of Fig. 5.7C. Here, the difference between mean phase of field $\mathrm{A}$ and $\mathrm{B}$ is $3.12 \mathrm{rad}$, which equals to $8.89 \mathrm{~mm}$ differential vertical movement of field A with respect to field B. Since a full $2 \pi$ cycle equals $17.9 \mathrm{~mm}$ vertical motion, and the phase is wrapped modulo $2 \pi$, this differential movement can either be $8.89 \mathrm{~mm}$ differential subsidence (shrinkage) or $9.01 \mathrm{~mm}(17.9-8.89)$ uplift (swell) of A with respect to B. From the in situ data in Fig. 5.2 we can calculate the double-difference shrinkage to be $7.6 \mathrm{~mm}$ for the upper $100 \mathrm{~cm}$ of the soils, which corresponds fairly well to the InSAR estimate interpreted as $8.89 \mathrm{~mm}$ shrinkage of 


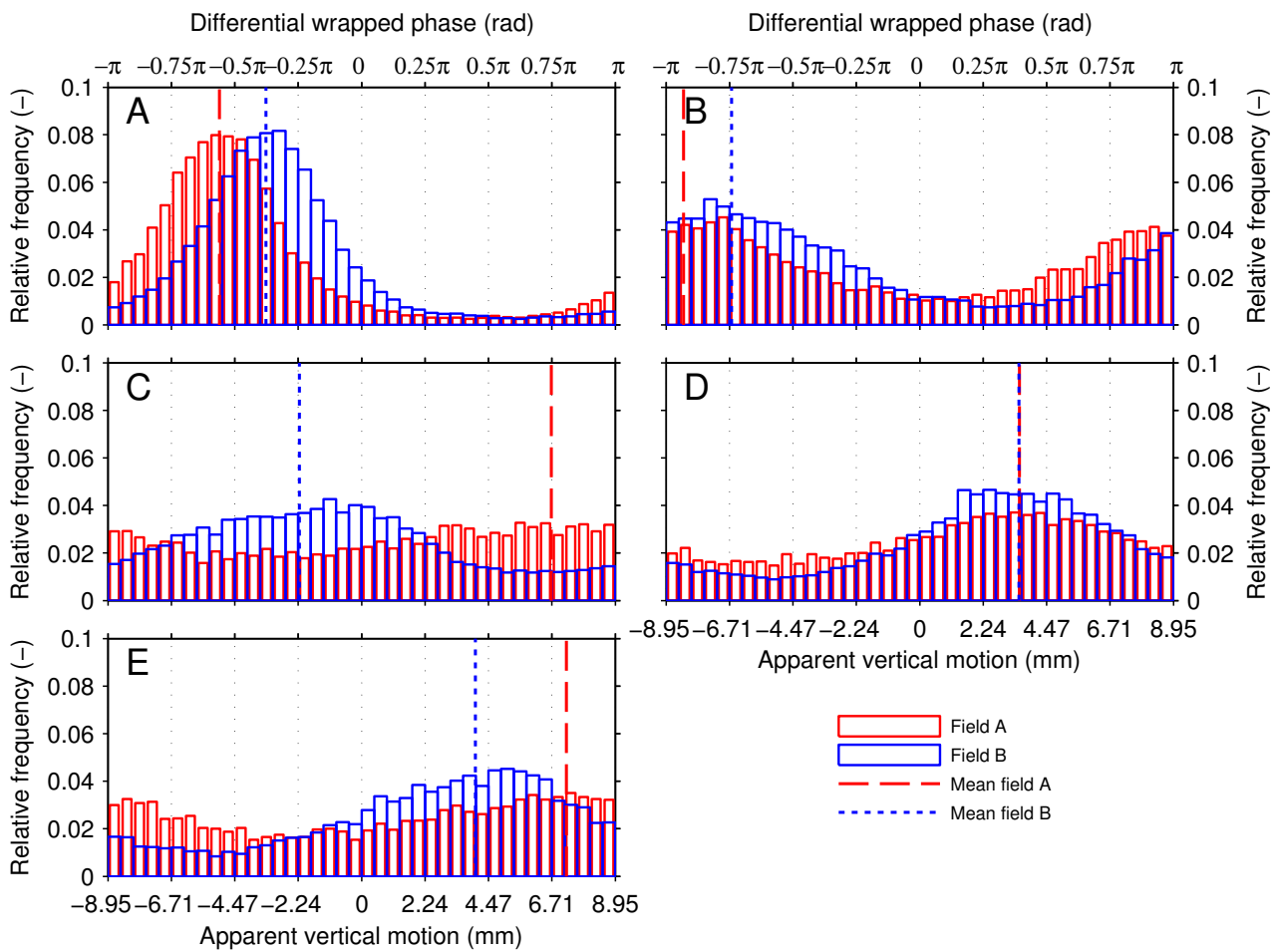

Fig. 5.7 Histograms of interferometric phase over field A and B from cascade processed TerraSAR-X data from 2011. Acquisition dates were: 17 Mar., 28 Mar., 19 Apr., 11 May, 22 May, and 2 June. Histogram A is calculated from the oldest interferogram (master: 17 Mar., slave: 28 Mar.) and $\mathrm{E}$ from the youngest (master: 22 May, slave: 2 June). Histograms show wrapped phase between $-\pi$ and $\pi$ on the top x-axes, and equivalent apparent vertical motion on the bottom x-axes. Dashed vertical lines indicate the mean phase values per field after multilooking over the entire agricultural field.

field A with respect to field $\mathrm{B}$. This agreement is obtained, despite the loss of coherence and multilooking therefore seems to be an appropriate step to reduce phase noise.

A comparison between double difference (multilooked) phase change and double difference in situ measured surface elevation changes of field A with respect to field B is shown in Fig. 5.8. In this figure the sign of the phase changes have been changed, so that positive phase represents swell, and negative phase change represents shrinkage. The top panel of Fig. 5.8 shows phase difference and in situ measured elevation change differences per interferogram, while the bottom panel shows cumulative differences from 17 March to 2 June. All presented phase data so far was 'wrapped' phase on a scale between $-\pi$ and $\pi$. In Fig. 5.8 phase unwrapping is based on the available in situ data. In 4 out of 5 cases, the direction of in situ vertical movement and most probable unwrapped phase value agree well. Both phase changes and in situ double difference elevation changes are generally small, except at 11 May. 


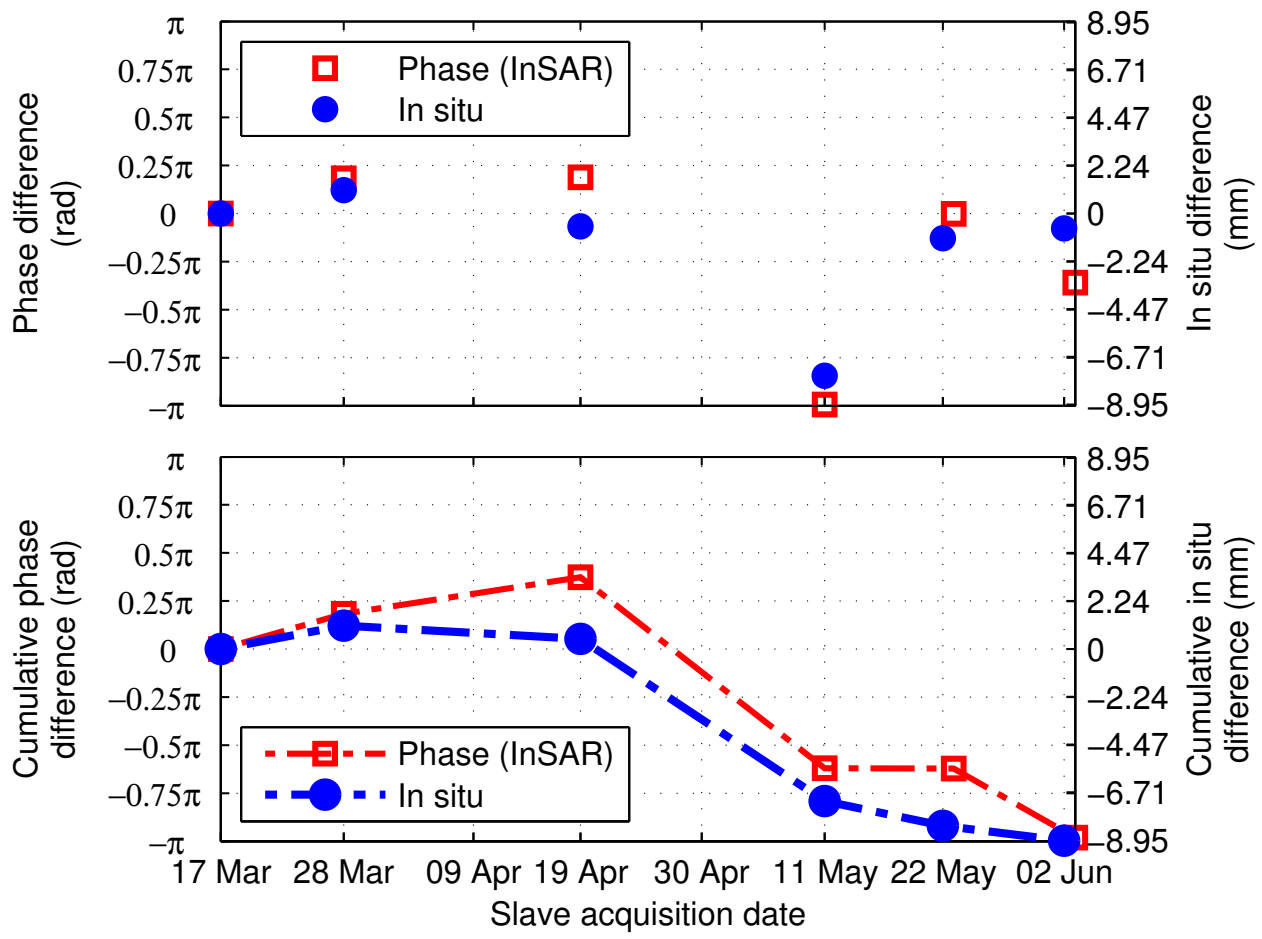

Fig. 5.8 Double differences phase change and double difference in situ measured surface elevation change of field A with respect to field B at slave acquisition dates (top) and cumulative double differences of phase and in situ measured surface elevation changes (below). The sign of phase change has been changed, so that positive phase represents swell, and negative phase change represents shrinkage.

\subsection{Discussion}

The occurrence of a soil moisture phase signal is a point of discussion in InSAR applications over natural terrain. In this chapter we solely considered geometric effects as a result of swelling and shrinkage of clayey soils. However, other soil moisture phase effects have been suggested, the most prominent being penetration (Nolan \& Fatland, 2003) and dielectric (Bhattacharyya et al., 2007; Rabus et al., 2010; De Zan et al., 2014; Zwieback et al., 2015) phase effects. According to Nolan \& Fatland (2003), penetration depth depends on the moisture content of the upper centimetres of the soil, where an X-band signal penetrates about $5 \mathrm{~mm}$ into the soil at a realistic lowest volumetric water content of $10 \%$ (assuming an uniformly distributed soil moisture with depth and taking into account transmission losses). No penetration was found at volumetric water contents larger than $32 \%$, meaning that the realistically large soil moisture change would cause a maximum apparent deformation of $5 \mathrm{~mm}$. According to De Zan et al. (2014) changes in penetration depth between master and slave image would contribute to coherence loss, and not to interferometric phase, since deeper 
penetration will introduce 'new' scatterers. Based on model results the authors suggest that, phase changes are caused by a change in propagation effects due to soil moisture induced dielectric changes. This was supported by analysis of L-band data by Zwieback et al. (2015). Penetration changes would only play a role in weighting the coherent scatterers. Ben Khadhra et al. (2006) however, argue that measured phase signals from bistatic X-band measurements in a controlled anechoic chamber could not be explained by pure dielectric effects and several authors have reported the relation between phase and moisture content to be dependent on surface roughness and the shape of the moisture profile (Nesti et al., 1998; Rabus et al., 2010). In this study the occurrence of penetration or dielectric effects in the observed phase signals could not be ruled out, neither quantified.

Penetration and geometric effects of soil moisture will both result in the same direction of phase change; a shift towards larger phase upon drying. The dielectric effect however, would result in a lengthening of the apparent path length upon wetting, and will therefore be oppositely directed to effects of penetration and geometric changes (Rabus et al., 2010; De Zan et al., 2014; Zwieback et al., 2015). Rabus et al. (2010) reported that variations of $27 \%$ volumetric water content within a soil layer of $2 \mathrm{~cm}$, were able to produce phase changes of more than $30^{\circ}$, or $0.53 \mathrm{rad}$ at $\mathrm{HH}$ polarization (as used in our study). Although observable by InSAR, this phase is much smaller than the phase and vertical shrinkage reported in this chapter. Since geometric phase signals include effects of soil moisture variations cumulative over the entire unsaturated zone, phase signals as a result of swelling or shrinking clays is probably dominant in case of simultaneous occurrence of different soil moisture phase contributions.

In this study phase changes over two agricultural fields are compared to double difference in situ measurements to illustrate the potential of InSAR. In hydrological studies, the temporal changes of a point or area is usually of primary interest, and double-differences are not useful as such. Double-differences also hamper comparison with in situ data (e.g. levelling of soil surface elevation or soil moisture content measurements) or model output. By relating InSAR double differences $d d$ to a nonmoving target $\mathrm{C}$ in an interferogram (assuming no local phase change of $\mathrm{C}$ ), they can be converted to single differences $d$ and the phase change of the (unknown) reference pixel $r$ is cancelled out:

$$
d_{A}^{m s}=d d_{r A}^{m s}-d d_{r C}^{m s} .
$$

In this way the InSAR observations can be compared to in situ data, and time series of phase change can be constructed from multiple cascade-processed interferograms. Selection of the stable target is important in this procedure. A stable reference target must not experience any phase change from the source of interest (e.g. swell and shrinkage of clayey soils), nor phase change that is absent in the targets or areas of interest. Care should be taken to avoid phase differences between the points of interest and the reference target resulting from atmospheric effects, specific scattering properties (e.g. double bounce scattering or dielectric effects) or thermal dilation and uncorrected topographic phase (e.g. in case the target is a high building). The target preferably is a stable and coherent point scatterer in multiple interferograms, so that multi-temporal interferometry techniques (e.g. Ferretti et al., 2001) can be used to 
model its temporal evolution in order to limit atmospheric effects and noise. If this condition is met, targets mounted below a clay layer or in the saturated zone of the soil, could help to isolate phase change due to the actual layer thickness change of the unsaturated zone from phase change from possible deeper sources (e.g local deformation due to mining activity or deep ground water extraction). Corner reflectors (e.g. Marinkovic et al., 2008) or Compact Active Transponders (CATs, Mahapatra et al., 2014) could serve as such well-defined benchmarks. These devices are designed to obtain coherent InSAR observations and their set up can therefore be designed to meet requirements for a specific purpose of study. The possibilities and accuracy of selecting stable reference points by multi-temporal interferometry techniques, largely depends on the number of SAR acquisition available.

For phase unwrapping in the example of clay swell and shrinkage presented here, widely available precipitation and potential evapotranspiration data could serve as an indication of drying (shrinkage) or wetting (swell), to determine the direction of phase change. Qualitative descriptions, rather than exact magnitude of precipitation or evapotranspiration fluxes, can already aid considerably as was demonstrated in Fig 5.2, where a clear relation is observed between P-ET and clay layer thickness changes. More general, the combined use of multiple wavelengths SAR sensors over the same area could aid in deformation estimation and phase unwrapping (Morishita \& Hanssen, 2015b), since the sensitivity to deformations varies with wavelength.

Although the land use and soil maps used in this study are highly simplified and only a few interferograms are analysed here, the examples show that complex patterns in InSAR data can be related to surface characteristics over agricultural area. To use InSAR to study unsaturated zone processes, the most favourable conditions for good phase observations need to be identified. Besides, the InSAR observations need to be related to a specific surface feature in order to be fully applicable in hydrological studies.

The short repeat cycle of TerraSAR-X, together with the sensors high sensitivity to deformations (largely determined by its short wavelength) was expected to fit the time scale and magnitude of clay swelling and shrinkage, although X-band SAR is relatively prone to temporal decorrelation compared to longer wavelengths. This is indeed found to be the case, although failure of acquisitions, causing temporal baselines to become longer than 11 days, hamper good phase estimates during the growing season. This indicates the importance of high temporal resolution of SAR data and need for accurately planned acquisition schedules. Multilooking obtains reasonable phase estimates over partly decorrelated areas, but high quality phase estimates are hard to obtain over vegetated areas. The value of applying multilooking is expected to increase with longer wavelengths, and smaller temporal baselines. Unmatched temporal resolution of C-band SAR is currently being provided by Sentinel-1 at medium high spatial resolution, but was not available yet in the period the field campaign in the Purmer area was conducted. 


\subsection{Conclusions}

InSAR measurements of unsaturated zone processes can potentially provide muchneeded observations at the field scale and beyond. We showed that phase observations over agricultural fields can be related to small surface elevation changes as a result of frost heave and shrinkage of clayey soils. Interferometric phase and in situ measurements of clay shrinkage revealed promising agreement, which could be applied to estimate soil water storage depletion using relations between water storage change and soil surface elevation change (Ch. 3 and 4).

Challenges and limitations inherent to InSAR and more specifically relevant for applications over agricultural areas were reviewed and identified. The challenges and limitations underline the need for careful selection of agricultural fields or areas to base InSAR analysis on. We showed that quantification of temporal evolution of interferometric phase and coherence changes associated with different vegetation types, soil types and time of year, can contribute to this selection. Agricultural crop fields offer the best phase estimates in winter, while grass fields are more coherent in summer. Some agricultural fields appear coherent at moments during the summer, which may depend on crop type.

With the increasing quantities of available data with different properties (e.g. polarimetric SAR data to assess vegetations structure and scattering behaviour) to evaluate, InSAR could become a valuable remote sensing technique for observations of unsaturated zone processes on larger scales. The use of advanced processing techniques obtaining combining phase from both point scatterers and distributed scatterers (e.g. Ferretti et al., 2011; Samiei-Esfahany et al., 2016), can further increase the applicability of InSAR for this purpose. 



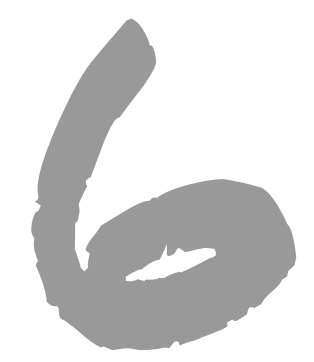

\section{InSAR estimates of clay dynamics related to soil moisture}

\subsection{Introduction}

In this chapter we study the interferometric phase over an agricultural area with clay holding soils in the Netherlands. We consider two mechanisms related to soil moisture content in clay soils that potentially affect interferometric phase: the clay swelling and shrinkage mechanism, causing actual deformations as a result of soil moisture content variations (Gabriel et al., 1989; Nolan \& Fatland, 2003; Zwieback et al., 2015; Bonì et al., 2016b, and Ch. 5 in this thesis), and the soil dielectric mechanism, based on propagation of the electromagnetic wave related to soil moisture content (Bhattacharyya et al., 2007; Rabus et al., 2010; De Zan et al., 2014; Zwieback et al., 2015). The goal is to estimate vertical deformation as a result of clay shrinkage, and thereby apply corrections for unwanted signals like soil moisture dielectric phase (called phase reduction) and improve temporal phase unwrapping. To do so, we apply Equivalent Single Master (ESM) phase estimation (Samiei-Esfahany et al., 2016), a simplified soil shrinkage model, and derived soil moisture phase effects based on known soil moisture depletion and soil properties. The use of contextual data for phase reduction and unwrapping is highlighted for measuring vertical clay dynamics, or for correction in other InSAR applications. This study is relevant for hydrological monitoring as surface elevation change measurements from clay soils might serve as

This chapter is a modified version of: Te Brake, B., Samiei-Esfahany, S., Van der Ploeg, M.J., and Hanssen, R.F.: InSAR estimates of clay dynamics related to soil moisture. In preparation. 
a proxy for soil water storage change (Ch. 4), especially on larger spatial scales. From an InSAR perspective, the relevance lies in improved understanding and insight in the significance of mechanisms that affect phase observations and phase unwrapping.

This chapter is organized as follows. The study area and used data are described in Sec. 6.2. InSAR methodology, including processing choices, the ESM-phase estimation procedure, and the unwrapping procedure are described in Sec. 6.3. Sec. 6.4 explains the computation of the soil moisture dielectric phase to reduce the observed phase and separate the effects of the soil shrinkage and soil dielectric mechanisms in the InSAR observations. The results of the phase reduction and shrinkage estimation are shown and discussed in Sec. 6.5. In Sec. 6.6 we propose a shrinkage model based on contextual data to aid phase unwrapping. The main results are summarised and discussed in Sec. 6.8.

\subsection{Study area, in situ measurements, and radar data}

Field observations and SAR images from the twin satellites TerraSAR-X (TSX) and Tandem-X (TDX), were obtained for the Purmer area, approximately $15 \mathrm{~km}$ north of Amsterdam, the Netherlands (Fig. 6.1). For the interferometric phase analysis an area with 26 agricultural fields is considered. Fields are of rectangular shape of approx. 700 by $80 \mathrm{~m}$. Some fields are permanently covered with grass, but most are used for crop rotation of winter wheat, potatoes, sugar beets, and maize and have no crop cover in winter.

In situ measurements of soil moisture and surface elevation change were taken on a grass field, labelled as field 9 in this chapter ${ }^{1}$, centrally located among the fields under consideration. Because of availability of in situ measurements, we primarily focus on this field. The upper $100 \mathrm{~cm}$ of the soil at field 9 contains about $19 \%$ clay (65\% montmorillonite, $25 \%$ illite, $10 \%$ kaolinite). The sand fraction varies between 9 and $26 \%$, with the upper $15 \mathrm{~cm}$ of the soil containing $21 \%$ sand (Ch. 3, field B). Soil moisture content of the upper soil layer was measured using a Campbell Scientific CS616 sensor installed at $9 \mathrm{~cm}$ depth. Meteorological data were obtained from measurement stations of the Royal Netherlands Meteorological Institute (KNMI) in Edam (precipitation, $2 \mathrm{~km}$ from the study area) and Berkhout (reference potential evapotranspiration, $15 \mathrm{~km}$ from the study area). The area, in situ measurement methods, and in situ data are described in detail in Ch. 4. Measured soil moisture content and vertical deformation are presented in Fig. 6.2.

For the InSAR processing a total of 19 radar images from the TerraSAR-X and Tandem-X satellites, acquired between late October 2010 and early June 2011, are used. This particular period is chosen since for this period in situ data are available, soil moisture content and surface elevation vary considerably, and to avoid significant decorrelation effects in the summer period caused by crop cover and tillage.

${ }^{1}$ In Ch. 3 to 5 this field is labelled as field B. Since we consider 26 fields in this chapter, the labelling has been adapted. 


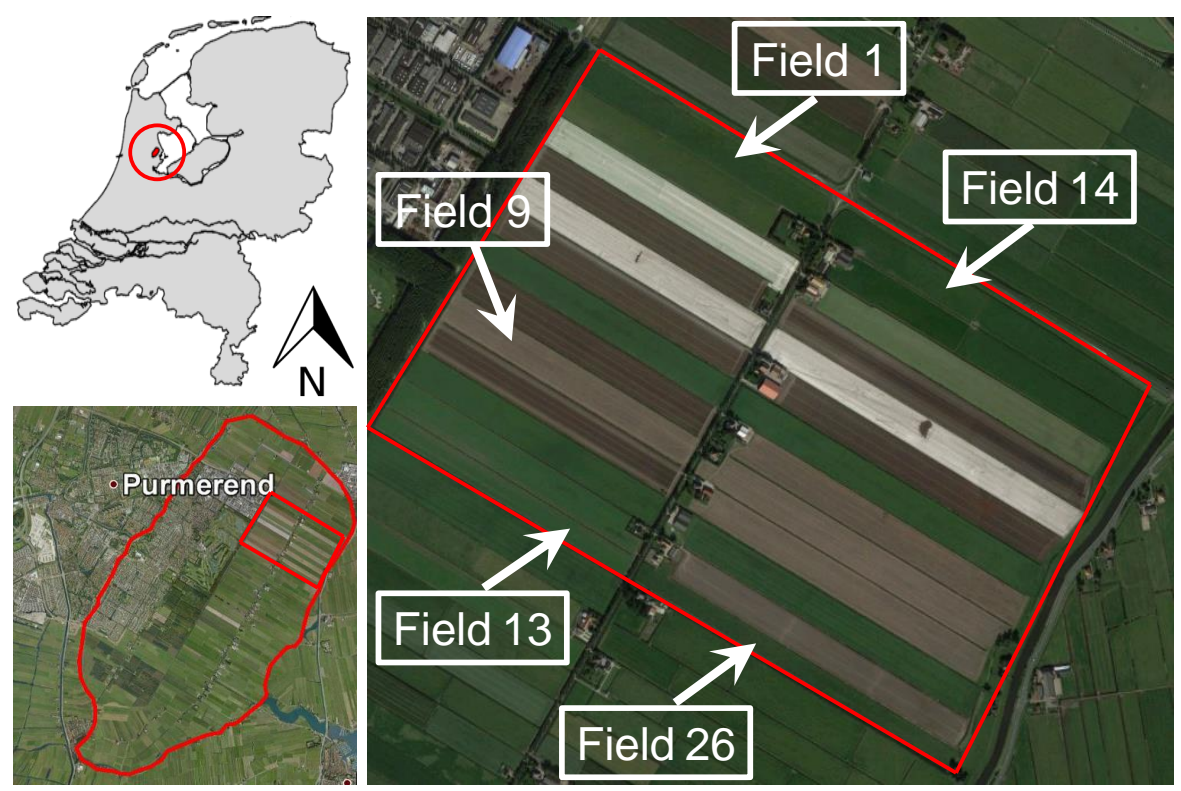

Fig. 6.1 Location of the study area in the Netherlands, and location of the studied fields within the area. Fields are located along a road, with field 1 to field 13 on the west side from north to south, and field 14 to field 26 on the east side. Map data: (C)2016 Google (https://www.google.com/earth/).

\subsection{Methodology}

Multi-master interferograms are computed, including the removal of the reference phase and topographic phase using data from the Shuttle Radar Topography Mission (SRTM). To obtain ESM-phase time series of clay shrinkage, the processing steps below are taken and described in the following sections:

1. Multilooking and coherence estimation (Sec. 6.3.1);

2. ESM-phase estimation (Sec. 6.3.2);

3. Spatial phase referencing (Sec. 6.3.3);

4. Phase unwrapping (Sec. 6.3.4).

\subsubsection{Multilooking and coherence estimation}

Spatial filtering by means of multilooking is applied to reduce phase noise. Multilooking (spatial normalized coherent averaging) can be done over statistically homogeneous pixels (SHP, Ferretti et al., 2011), assuming no spatial variation of signal components. Here, spatial filtering is applied by confining multilook areas by field boundaries. In this way, a large number of pixels (approx. 9.000) is obtained, over which constant signal components of phase are assumed. Based on the homogeneity of land cover and tillage within a field, this assumption is justified. Heterogeneity in soil properties, like clay content, and depth of the unsaturated zone potentially affecting clay shrinkage and soil moisture content are however omitted. 


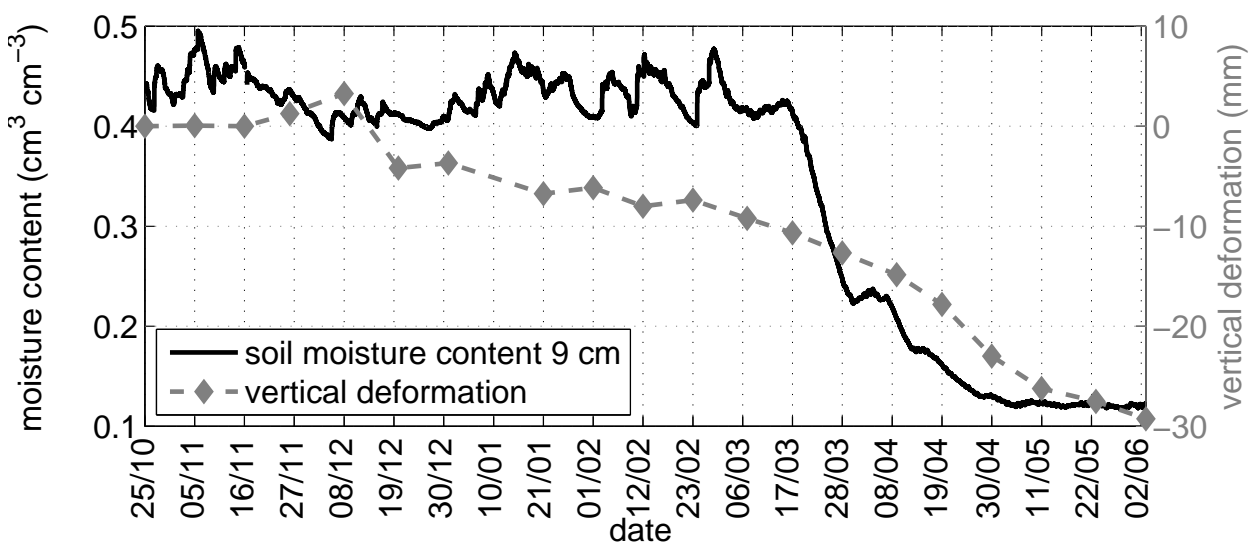

Fig. 6.2 Soil moisture content at $9 \mathrm{~cm}$ depth and vertical displacement of the upper $100 \mathrm{~cm}$ of the soil at field 9, between 25 Oct. 2010 and 3 Jun. 2011. Positive values of displacement indicate soil swelling, negative values indicate soil shrinkage.

After multilooking, a complex coherence matrix containing all interferometric combinations can be constructed for each multilooked pixel, i.e. agricultural field. The argument of the complex coherence is the interferometric phase, while the absolute value represents estimated coherence. Figure 6.3 shows the absolute coherence matrices of 4 fields. Over the agricultural fields under consideration coherence was generally low over long temporal baselines, but different patterns of temporal decorrelation were observed. Decorrelation patterns in Fig. 6.3 can be mostly explained from land cover, vegetation status, and tillage, as observed in the study area:

Fig. 6.3A Field 2, permanent cover of grassland, mowed after acq. 16 (19 Apr. 2011);

Fig. 6.3B Field 9, grass for seed harvesting, short grass throughout winter, growing season between May (acq. 17) and July;

Fig. 6.3C Field 20, sugar beets in autumn (dense cover with large leaves, harvested after acq. 3 (16 Nov. 2010), ploughed after acq. 4 (27 Nov. 2010)), bare throughout winter, cultivation and planting of potatoes on ridges after acq. 15 (28 Mar. 2011);

Fig. 6.3D Field 23, stubbles in autumn, ploughing after acq. 5 (8 Dec. 2010), bare throughout winter, cultivation in spring, sugar beets start to emerge at acq. 16 (19 Apr. 2011), so probably cultivation after acq. 14 (17 Mar. 2011).

At the beginning of the study period, some fields were still covered with rotational crops (e.g. potatoes and sugar beets, Fig. 6.4A), while other fields were already bare or covered with short grass. In December 2010 (acq. 5-7) fields where covered by snow (Fig. 6.4C), which seems to affect the coherence on all fields. To a lesser extend this was also observed in February (acq. 12, Fig. 6.4D). Tillage activities (such as ridging for potato planting, Fig. 6.4E) and mowing of grass cause coherence to drop suddenly, while crop emergence (Fig. 6.4F) and vegetation development (Fig. 6.4G-I) cause slow temporal decorrelation. Although field 2 and field 9 are both grass fields, the coherence patterns at these fields are somewhat different, with the coherence at 


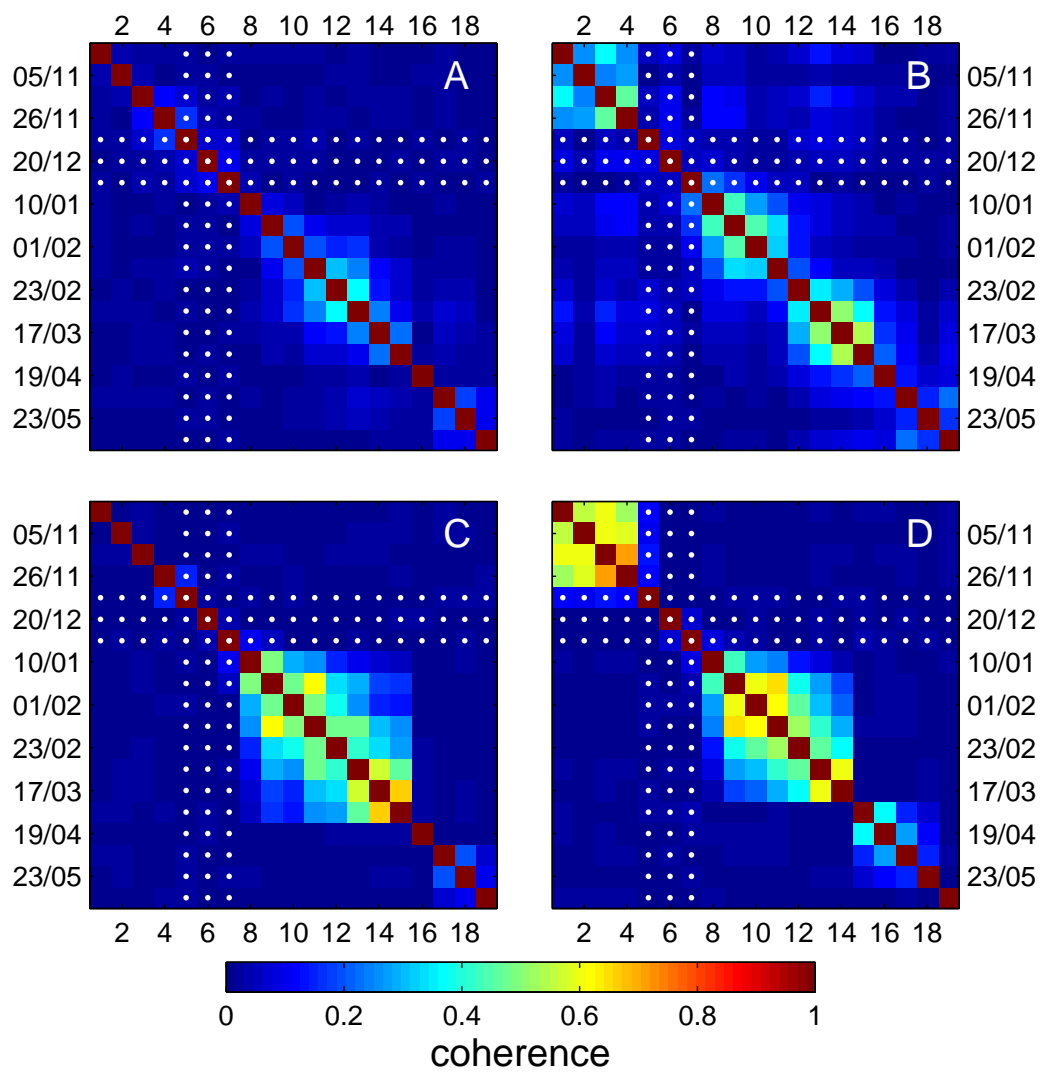

Fig. 6.3 Coherence matrices for 4 fields showing various patterns of coherence. A: field 2, permanent grassland; B: field 9, grass for seed harvesting; C: field 20, sugar beets to bare land to potatoes; D: field 23, stubbles to bare land to sugar beets. Snow affected acquisitions are indicated by white marks. Acquisitions are chronologically ordered and indicated by acquisition number on the $\mathrm{x}$-axis and acquisition date on the $\mathrm{y}$-axis.

field 9 generally being slightly higher. Field 2 is permanent grassland for cattle with dense plant cover (Fig. 6.4B), whereas field 9 is less densely covered with a different species of grass, intended for seed harvesting in July (Fig. 6.4G-I). The difference in structure and growing phases of the two difference types of grass might cause a difference in the coherence pattern.

\subsubsection{ESM-phase estimation}

To obtain time series of phase, we used integer least squares (ILS) ESM-phase estimation (Samiei-Esfahany et al., 2016). For the estimation, we use all the possible interferometric combinations of SAR images, in this case 171 interferograms (Fig. 6.5A). The propagation of information from each interferogram to the ESM-phase is guided by a weight matrix (Samiei-Esfahany et al., 2016), to assign different weights to different observations. Here, we used the square of the absolute coherence as weighting factor, 

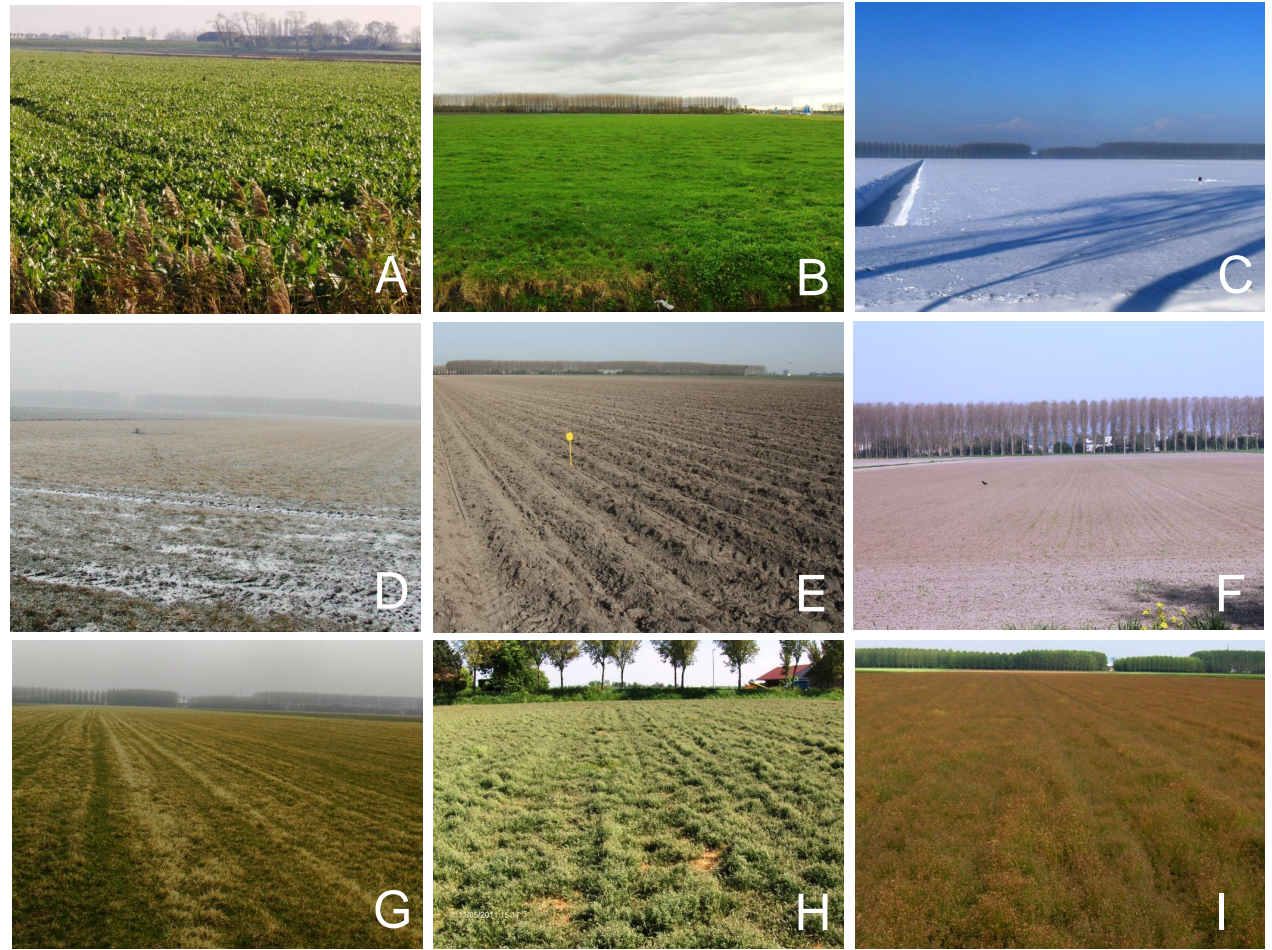

Fig. 6.4 Photos showing field status and development. A: sugar beet cover at field 20 in autumn; B: permanent grassland at field 2; C and D: snow and ice on field 9 in December and February; E: ridges for potato planting as done on field 20; F: sugar beet emergence on field 23; G-I: vegetation status at field 9 at 17 Mar., 11 May, and 3 June.

to assign high/low weight factors to interferometric phases with high/low coherence, respectively, without completely disregarding the information content of phases with low coherence. By applying the ESM-phase estimation step, the scattering noise effect can be reduced and a more precise phase time series is obtained, yielding improved parameter estimation and phase unwrapping. ESM-phase time series are estimated relative to 1 February 2011 (the equivalent single master, Fig. 6.5B).

\subsubsection{Spatial phase referencing}

To enable comparison with in situ data, the estimated ESM-phase time series are referenced to a stable target obtained from persistent scatterer interferometry (PSI) using the DePSI (Delft Implementation of PSI) algorithm (Van Leijen, 2014). In fact, a total number of 17 PS points on buildings near the field under consideration was selected based on low normalized amplitude dispersion index (threshold of 0.20) (Ferretti et al., 2001). Selected PS points are located on man-made structures close to the fields, so no signal related to the fields is expected to be present. To filter out noise, the phase of the selected points is averaged, and a synthetic reference point was created (Chang \& Hanssen, 2016). In fact, by using the synthetic reference 

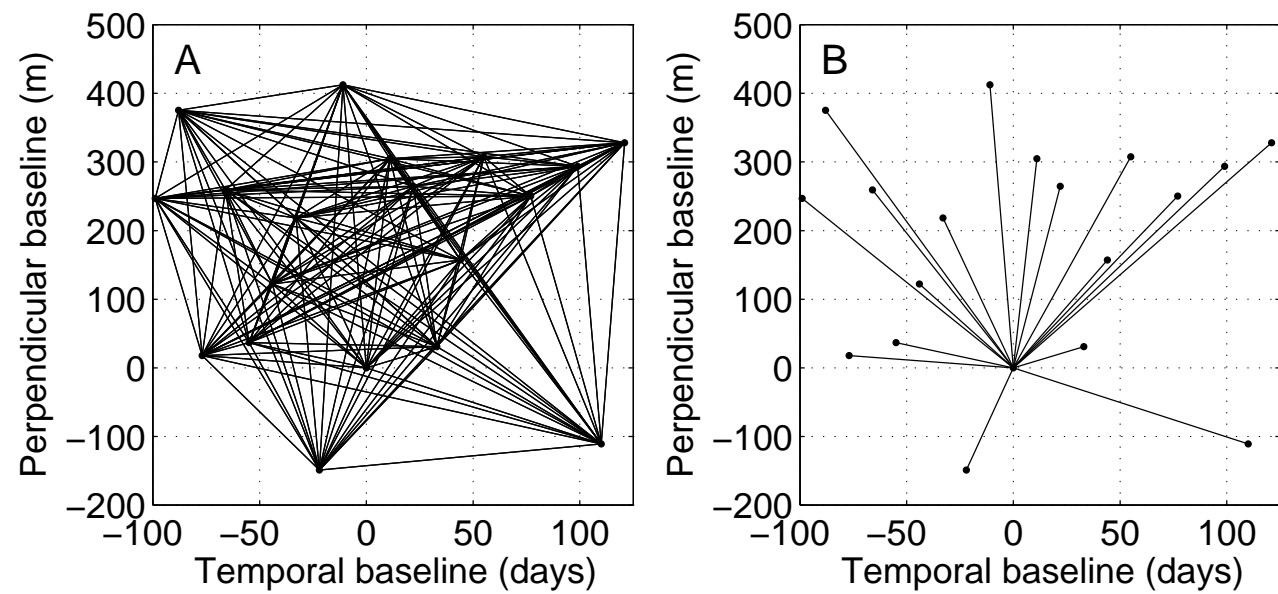

Fig. 6.5 Baseline configuration in the stack of 19 SLCs for the multi-master interferograms (A) and the equivalent single master (B) at 1 Feb. 2011.

point, we reference the fields to the average time series of the 17 nearby PS points, and in this way the the final time series are not affected by potential anomalies or autonomous displacements of one of these points. In this procedure, it is assumed that building movement, thermal dilatation, or other phase contributions specific for the PS points are absent on the agricultural fields. Unwanted phase is introduced from the height difference and a differential atmosphere effect between reference points and the agricultural field. Estimation of these contributions is accounted for in the temporal phase unwrapping procedure.

\subsubsection{Temporal phase unwrapping}

Temporal phase unwrapping of ESM-phase time series can be performed similar as done in PSI processing. Here we use the integer least squares estimator ${ }^{2}$ (ILS, Hanssen et al., 2001; Kampes \& Hanssen, 2004; Van Leijen, 2014). The ILS temporal phase unwrapping estimator jointly estimates the integer ambiguity cycle in the time series, the residual topography, and the parameters of the assumed deformation model. We assumed a quadratic model, based on the increasing clay shrinkage towards the end of the period under consideration, as measured in situ (Fig. 6.2).

\subsection{Soil moisture dielectric phase}

\subsubsection{Isolation of phase mechanism}

In the applied interferometric processing, the multi-master interferograms are corrected for phase contributions from unwanted sources, based on models or estimations, to isolate the signal of interest. Effectively, the applied corrections result in time series of unwrapped phase, originating from mechanisms on the agricultural field.

\footnotetext{
${ }^{2}$ Note that two different implementations of the ILS principle are used for i) ESM-phase time series estimation, and ii) temporal phase unwrapping.
} 
We consider two mechanisms related to soil moisture that affect the phase: the clay shrinkage mechanisms and the soil moisture dielectric mechanism. Using in situ data of soil moisture content, soil texture, and the model proposed by De Zan et al. (2014), we estimate soil moisture dielectric phase. This enables us to isolate the shrinkage phase, while assessing the relative contribution of soil moisture dielectric phase with respect to shrinkage phase. Corrections for other potential phase contributors over agricultural fields, like scattering phase and vegetation effects are not considered.

\subsubsection{Soil moisture dielectric phase estimation}

De Zan et al. (2014) developed a model to predict the interferometric phase based on empirical relations between the dielectric constant and soil moisture content. The fundamental concept of the model is that a change in soil dielectric constant (as a result of wetting or drying) corresponds to a change in propagation of an electromagnetic wave and thereby to the complex vertical wavenumber in the soil, modelled as a lossy medium. The real part of this complex wavenumber determines the change in phase over a certain depth (Zwieback et al., 2015; De Zan et al., 2015). De Zan et al. (2014) showed that their model works well over bare fields, based on inversion of soil moisture content from inconstancies in phase triplets of L-band SAR data. As we were interested in phase reduction of ESM-phase time series, we used forward modelling to obtain the expected phase based on the mean soil moisture content at $9 \mathrm{~cm}$ depth within two hours around the time of image acquisition. ESM-phase estimation relies on multi-master phases to estimate time series with respect to a single master. Therefore, we predicted the interferometric phase at each acquisition from the change in soil moisture content in two ways: 1) direct change with respect to 1 February (single master), and; 2) reconstruction of phase with respect to 1 February from phase between consecutive acquisitions (multi-master). The absolute volumetric soil moisture content at 1 February was $41 \%$, close to saturation.

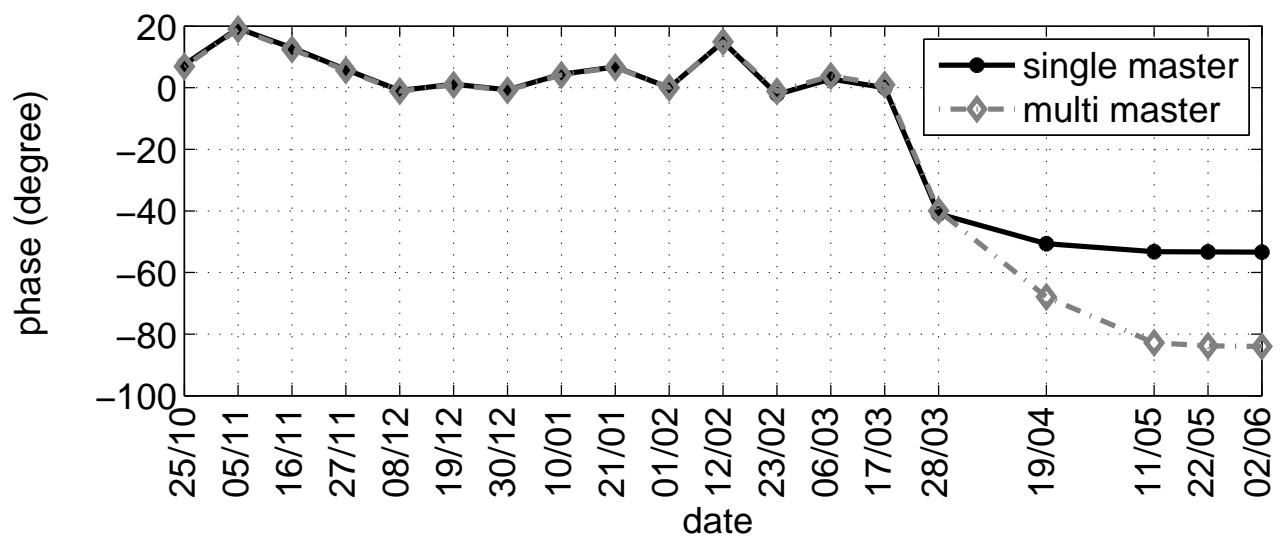

Fig. 6.6 Predicted soil moisture dielectric interferometric phase. All phase with respect to master at 1 Feb. 2011. 
The interferometric phase obtained from the soil moisture model ranges from $-53.4^{\circ}$ to $19.3^{\circ}$ for the single master case, and from $-84.0^{\circ}$ to $18.9^{\circ}$ for the multi-master case (Fig. 6.6). This corresponds to 2.7 to $-0.97 \mathrm{~mm}$ and 4.2 and $-0.75 \mathrm{~mm}$ of equivalent apparent vertical deformation (using the phase-to-height conversion based on wavelength $\lambda=0.031 \mathrm{~m}$, and incidence angle $\theta_{\text {inc }}=31.5^{\circ}$ ). There is a high sensitivity to small moisture content changes. As long as the soil moisture content at the master acquisition is close to $41 \%$, the results from the single master and multimaster reconstructed case are very similar. The modelled phase behaviour is however nonlinear (De Zan et al., 2014), causing a difference between the single master and multi-master reconstructed phase from 19 April, as from this moment the moisture content at part of the master acquisitions of the multi-master case is relatively low. The sensitivity of the modelled phase is higher in the lower moisture content range; thus a certain moisture content change results in a larger phase in the dry end, then that same absolute change does in the wet end (De Zan et al., 2014). The summation of multi-master phases therefore results in a larger total phase compared to the single master case. The observed difference (between single master and multimaster reconstructed phase) is the essence of the non-triangularity of the soil-moisture effect as exploited by De Zan et al. (2014). For comparison with ESM-phase, we will further use the predicted soil moisture dielectric phase from the single master case. The largest jump in phase is observed at an absolute soil moisture content change of $-17 \%$ at 28 March. The dielectric phase is directed opposite to shrinkage phase; upon drying dielectric phase decreases, while shrinkage phase increases due to a longer optical path between sensor and target (soil surface). This difference in direction was also observed by Zwieback et al. (2015). Penetration phase (as proposed by Nolan et al., 2003; Nolan \& Fatland, 2003) is not considered, because the effect of penetration provides no deterministic measure as new scatterers emerge upon deeper penetration (Morrison et al., 2011). Therefore it would only result in coherence loss (De Zan et al., 2014).

\subsection{Shrinkage estimation}

Time series of unwrapped ESM-phase are converted to vertical deformation and the simulated dielectric phase is subtracted. The resulting time series are interpreted as vertical deformations due to clay shrinkage, without a direct soil moisture phase contribution, and are compared to in situ measured vertical deformations from clay shrinkage.

The comparison between ESM-phase estimates of deformation and in situ data is presented in Fig. 6.7 for field 9. Error bars on ESM-phase time series represent the $2 \sigma$-confidence interval, using the standard deviation computed as the square root of the Cramér-Rao bound (Monti Guarnieri \& Tebaldini, 2008; Samiei-Esfahany et al., 2016). This is the theoretically lowest achievable standard deviation, which is assumed to be approached by multilooking over approx. 9.000 pixels. Note that these errorbars only account for the remaining scattering noise in the time series, but not for other kinds of errors, such as remaining atmospheric signal or height residuals. Therefore, the InSAR errorbars should be interpreted as the very lower bound of confidence 


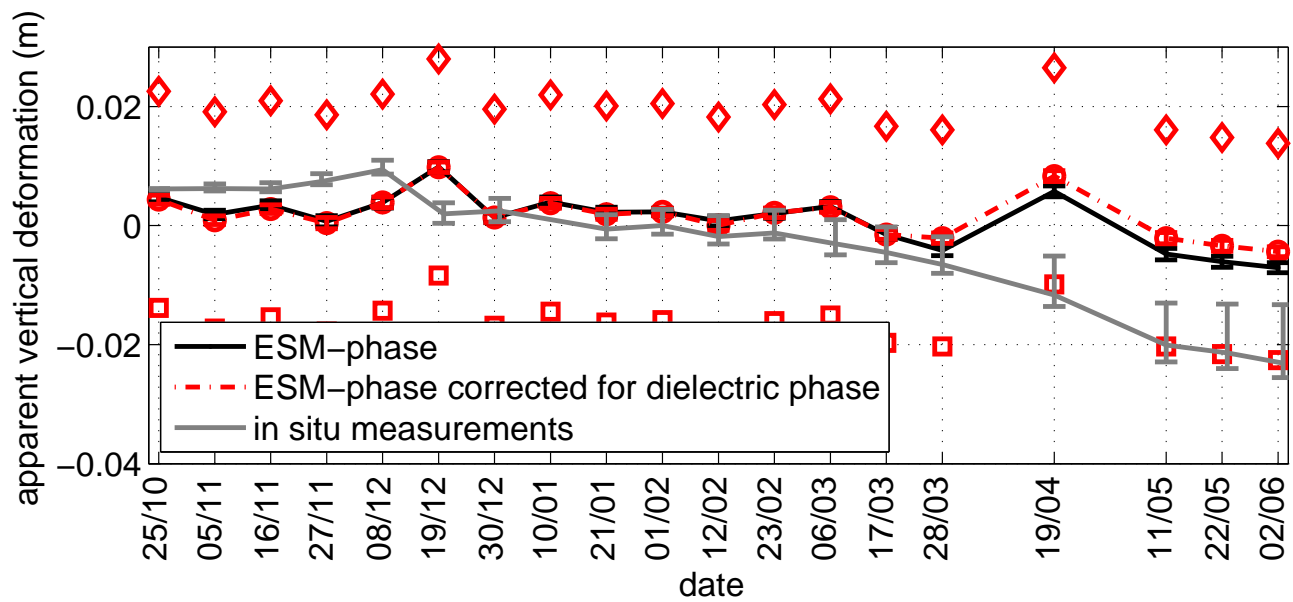

Fig. 6.7 Estimated deformation time series from ESM-phase, ESM-phase corrected for dielectric phase, and in situ measurements of vertical shrinkage on field 9. Unwrapping possibilities are indicated for the corrected ESM-phase by plotting estimated phase plus and minus $2 \pi$ phase cycles (half the wavelength, converted to the vertical direction).

interval, whereas the real error bar is larger. Error bars on in situ data are derived from measurement device accuracy, deviation due to a time difference between the TSX/TDX acquisition and the in situ measurement moment and weather conditions during this time, and error propagation between measurements in case of cumulative in situ measurements. This results in a rather arbitrary range, but is believed to approximate a $95 \%$ confidence interval of the point measurements. The measurement procedure is described in Ch. 4.

Estimated deformation and measured shrinkage in Fig. 6.7 do not correspond well in the beginning of the time series. Possibly the snow cover on the field hampers reliable phase estimation, or deformation due to frost heave, as observed in Ch. 5, is not captured well in the in situ measurements. More importantly, the results clearly reveal an unwrapping error in the period with considerable shrinkage (19 April). As the applied unwrapping model does assume a temporally smooth signal, it does not suffice to capture the sudden phase jump between 28 March and 19 April. The indication of the $2 \pi$ phase cycles in Fig. 6.7, suggests a more probable unwrapping option. Considering this correction in unwrapping, it is apparent that the clay shrinkage is captured by ESM-phase estimation. The observed magnitude of clay shrinkage phase is much larger than the simulated dielectric soil moisture phase, but the dielectric phase corrected ESM-phase matches the in situ measurements even better than the uncorrected ESM-phase. It seems that the soil moisture dielectric phase correction improves clay shrinkage phase estimation, but nonetheless unwrapping needs improvement. We propose a shrinkage model to aid unwrapping of the soil moisture corrected ESM-phase time series. 


\subsection{Unwrapping correction}

\subsubsection{Shrinkage model}

The observed phase unwrapping error for field 9 in Fig. 6.7 can be manually resolved because of the availability of in situ measured deformations. These kind of in situ data are however not generally available. Therefore, a quantitative description of clay dynamics (swell-shrink) based on general available data is beneficial to aid phase unwrapping. We propose a model to resolve vertical shrinkage based on hydrometeorological data and assumptions on shrinkage behaviour of the clay soil. The model capitalizes on the relation between soil water storage change $\Delta W$ and soil volume change per unit area $\Delta V$, described in Ch. 4. Assuming basic shrinkage, $\Delta W$ equals $\Delta V$ (Bronswijk, 1991b). Upon first drying however, water loss will not result in soil shrinkage yet, owing to structural shrinkage (Yule \& Ritchie, 1980a). Including $S$ for water loss in the structural shrinkage phase, the relation between water storage change and volume change becomes:

$$
\Delta W=S+\Delta V .
$$

Assuming constant isotropic shrinkage, we can express $\Delta V$ in terms of vertical deformation $\Delta z$ as defined by Bronswijk (1991a):

$$
\Delta V=3 \Delta z-3 \frac{\Delta z^{2}}{z}+\frac{\Delta z^{3}}{z^{2}}
$$

where $z$ is the soil layer thickness in mm over which shrinkage is to be calculated. An appropriate approximation of $\Delta W$ and estimation of $S$ allows the derivation of $\Delta z$. Assuming no effect of variation in groundwater level and capillary fringe depth on shrinkage, the total amount of soil water storage change $\Delta W$ can be estimated from the precipitation deficit:

$$
\Delta W=P-E T,
$$

where $P$ is the precipitation and $E T$ is the reference potential evapotranspiration. The use of these hydrometeorological data is advantageous, as these can generally be obtained from meteorological institutes or easy to deploy weather stations.

$S$ was estimated for various soil layers in the Purmer area and it is found that all vertical shrinkage in the period under consideration originated from the top $60 \mathrm{~cm}$ of the soil (Ch. 4). Therefore $z$ is $600 \mathrm{~mm}$, and neglecting the effects of groundwater and capillary fringe seems justified, as these do not or hardly reach the upper $60 \mathrm{~cm}$ of the soil. Combining Eqs. 6.1, 6.2 and 6.3 yields:

$$
\frac{1}{z^{2}} \Delta z^{3}-\frac{3}{z} \Delta z^{2}+3 \Delta z=(P-E T)-S,
$$

or,

$$
\frac{1}{z^{2}} \Delta z^{3}-\frac{3}{z} \Delta z^{2}+3 \Delta z-(P-E T)+S=0 .
$$

Equation 6.5 has one real root. Equation 6.5 only provides a good estimation of 
vertical deformation if the soil experiences normal shrinkage, for which Eq. 6.1 holds. The range of normal shrinkage of clay aggregates can be a priori assessed by means of the soil shrinkage characteristic curve (Ch. 3), but differs for a field soil (Ch. 4).

From the in situ measurements, it is found that the correlation between volume change in 11 day intervals and cumulative $P$-ET, is highest if only $P$ and ET data of the last 7 days prior to the shrinkage measurement are considered. This result is applied in the calculation of $\Delta z$, as an empirical correction to account for less then normal shrinkage and a deviation of the actual evapotranspiration from the reference potential evapotranspiration, which are both likely to have occurred due to dry conditions.

\subsubsection{Unwrapping results}

Figure 6.8 shows the modelled $\Delta z$, unwrapped ESM-phase corrected for unwrapping errors by use of the proposed model, and in situ measurements of shrinkage. Unwrapping is successfully corrected by forcing the phase to the $2 \pi$-modulus nearest to the predicted vertical shrinkage from the model, with a maximum phase jump of $2 \pi$ with respect to the unwrapped ESM-phase as was presented in Fig. 6.7. The model is only used to aid unwrapping in the period that actual shrinkage is modelled, in this case from 6 March. Processes that affect the phase in the period with rainfall excess are not modelled and therefore the model cannot be used to unwrap the phase time series in these periods.

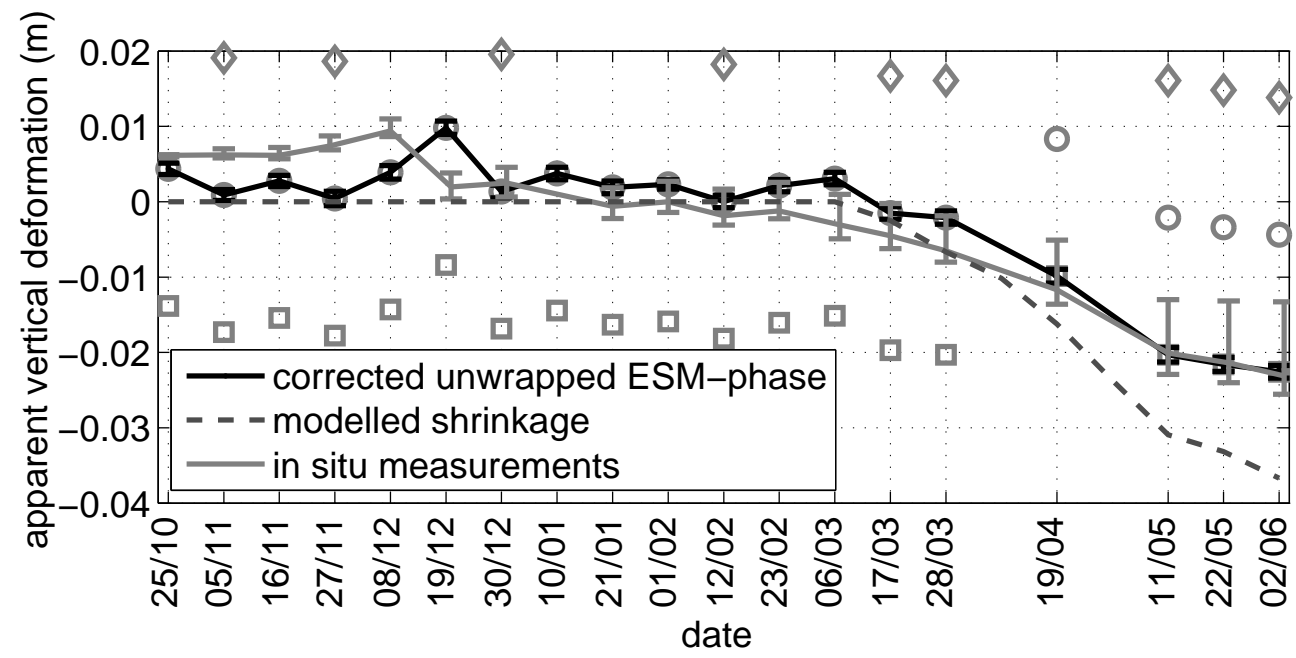

Fig. 6.8 Estimated deformation time series from ESM-phase corrected for dielectric phase and unwrapping errors, modelled vertical shrinkage, and in situ measurements of vertical shrinkage for field 9. 


\subsection{Shrinkage estimation on all fields in the study area}

The developed procedure of post-processing, phase unwrapping, phase reduction based on soil moisture content at field 9, and unwrapping correction based on the proposed shrinkage model, has also been applied to the other 25 fields in the study area. The resulting estimated shrinkage per field is shown in Fig. 6.9. Plots include ESMphase, with unwrapping possibilities indicated by estimated shrinkage plus and minus $2 \pi$ phase cycles. For offset correction and dielectric phase correction, data from field 9 are used. The figure titles indicate field number and land use during the period under consideration, including changes in land use from 2010 to 2011. In the transition between crops, the field is left bare for some time in all cases. In this case, bare does mean that the soil is not covered by crop; tillage may still be going on.

Shrinkage estimations from ESM-phase and unwrapping results cannot be verified without in situ measurements on the fields, but the patterns of phase time series suggest considerable shrinkage phase measured on various fields. Only for field 8 (field A in Ch. 3 and Ch. 5) some in situ measurements were available in spring 2011. The phase time series suggests somewhat larger maximum shrinkage on field 8 than on field 9, which is consistent with the in situ measurements as reported in Ch. 5. Phase patterns on various fields in the period without shrinkage might be partly explained by frost heave (Ch. 5). 

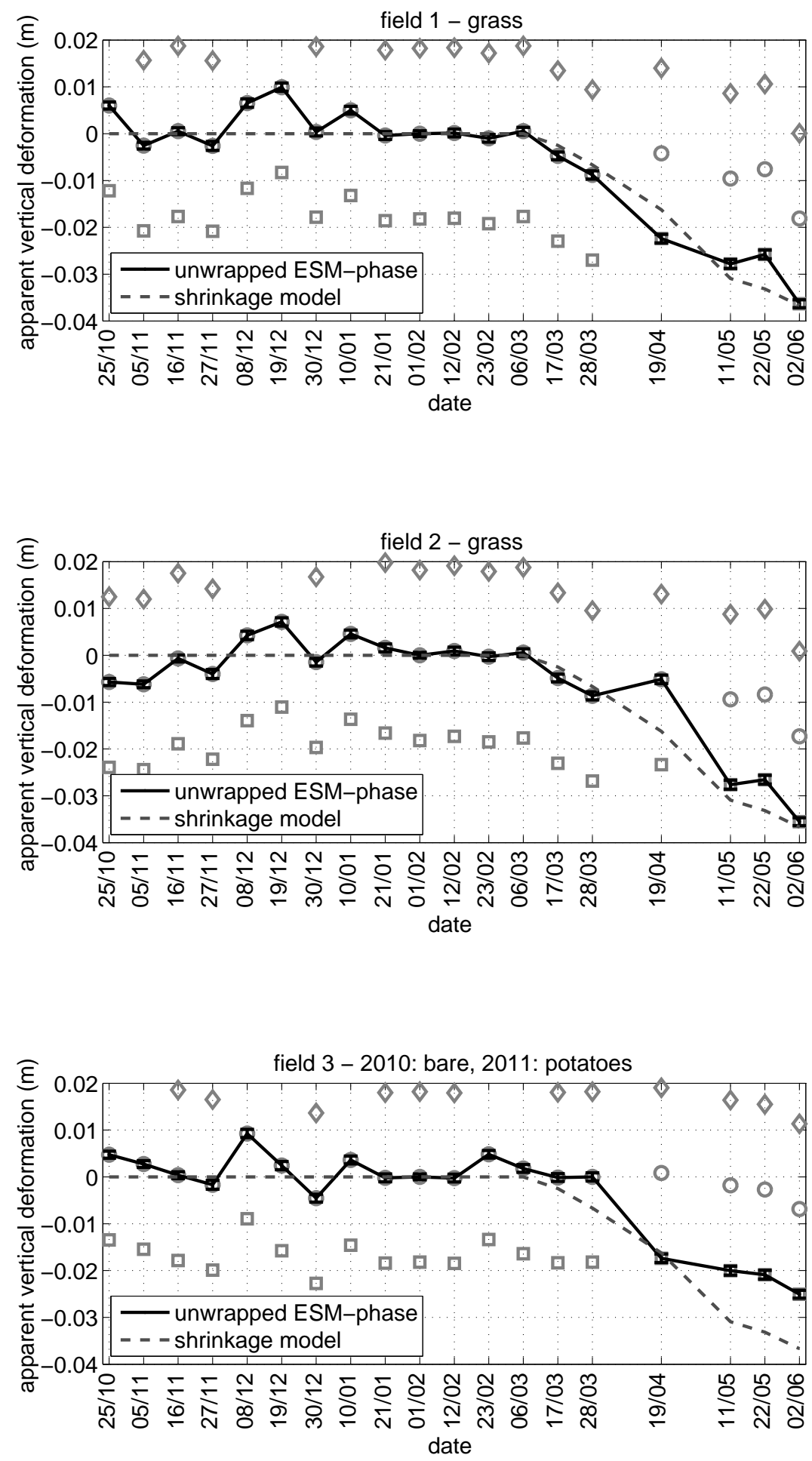

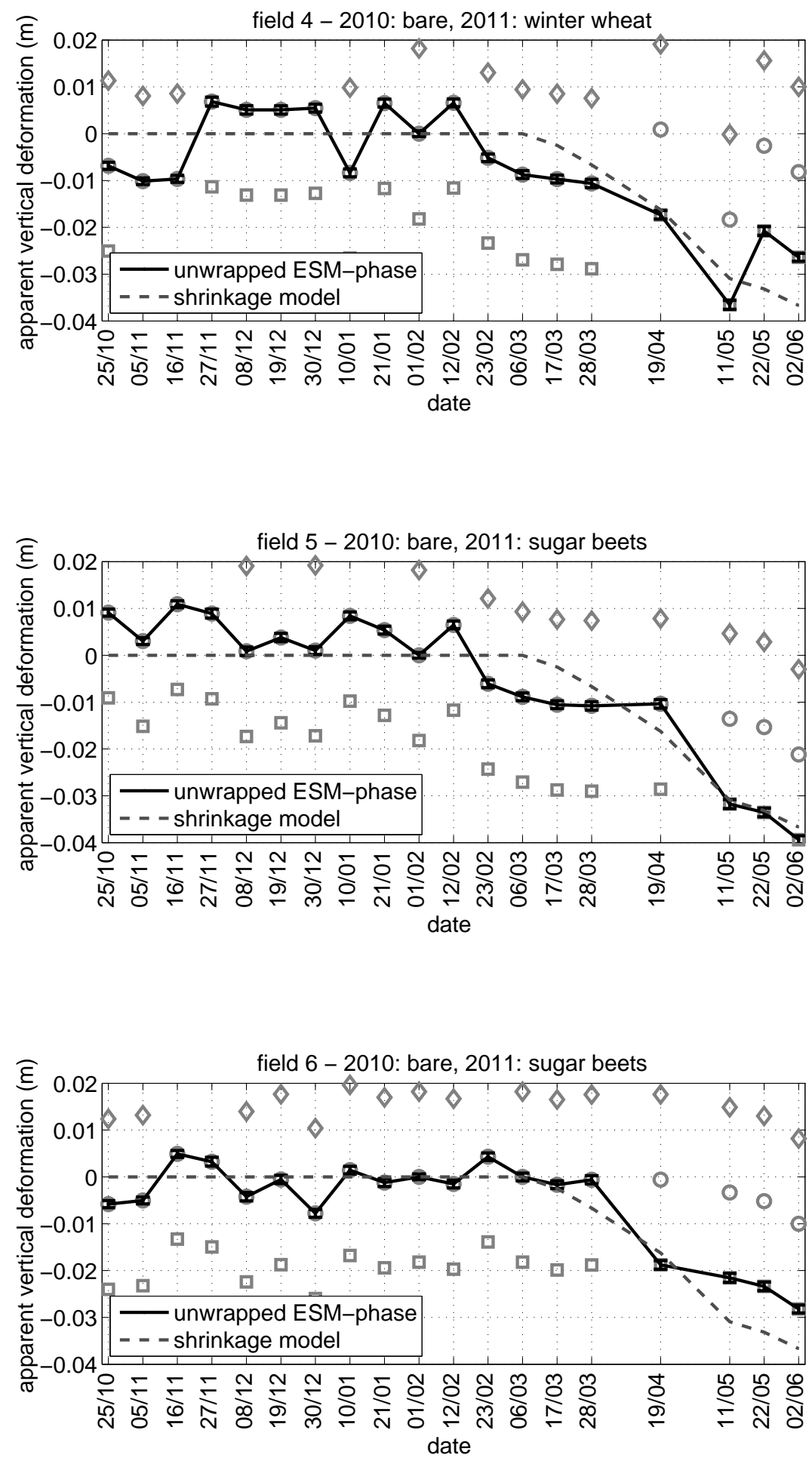

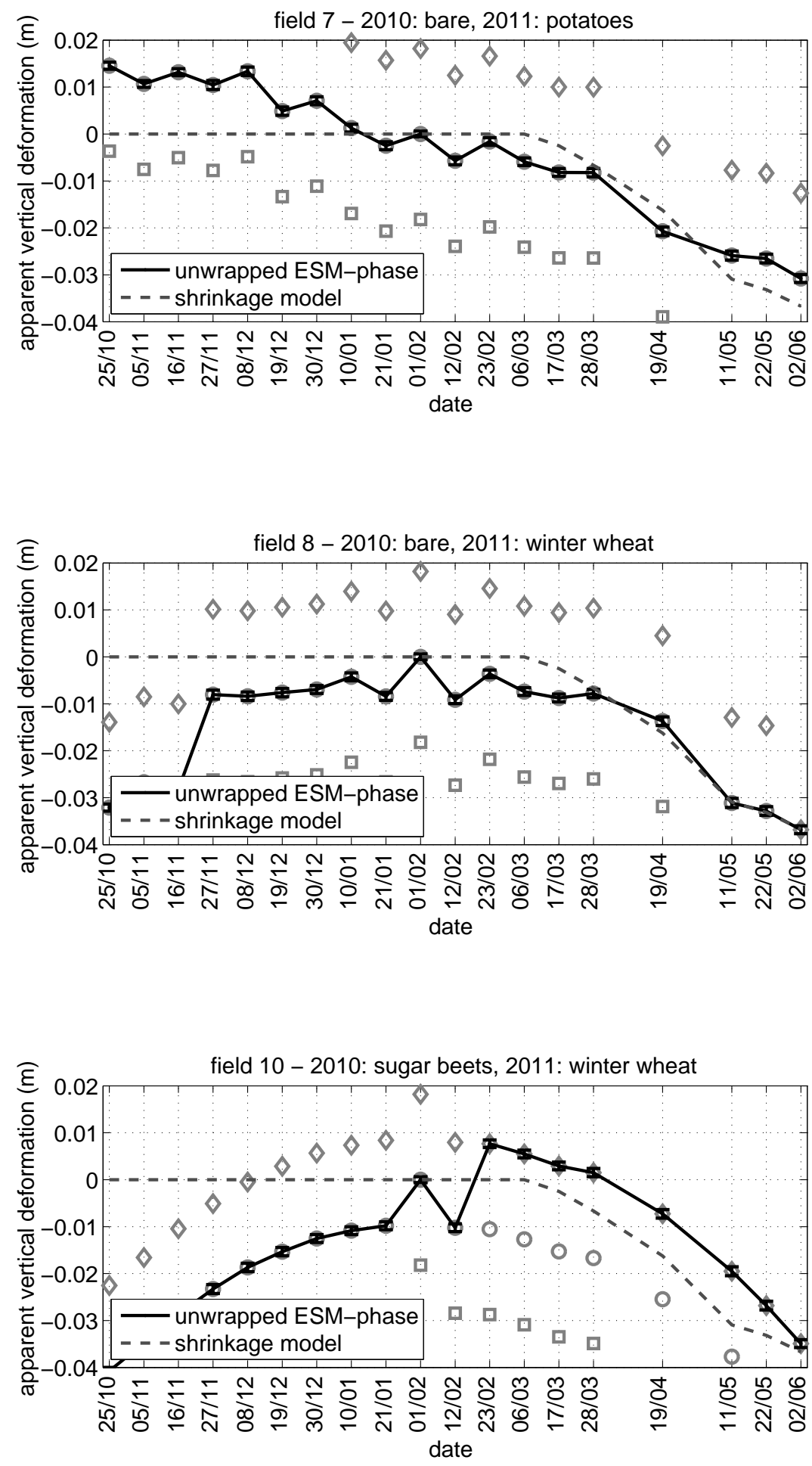

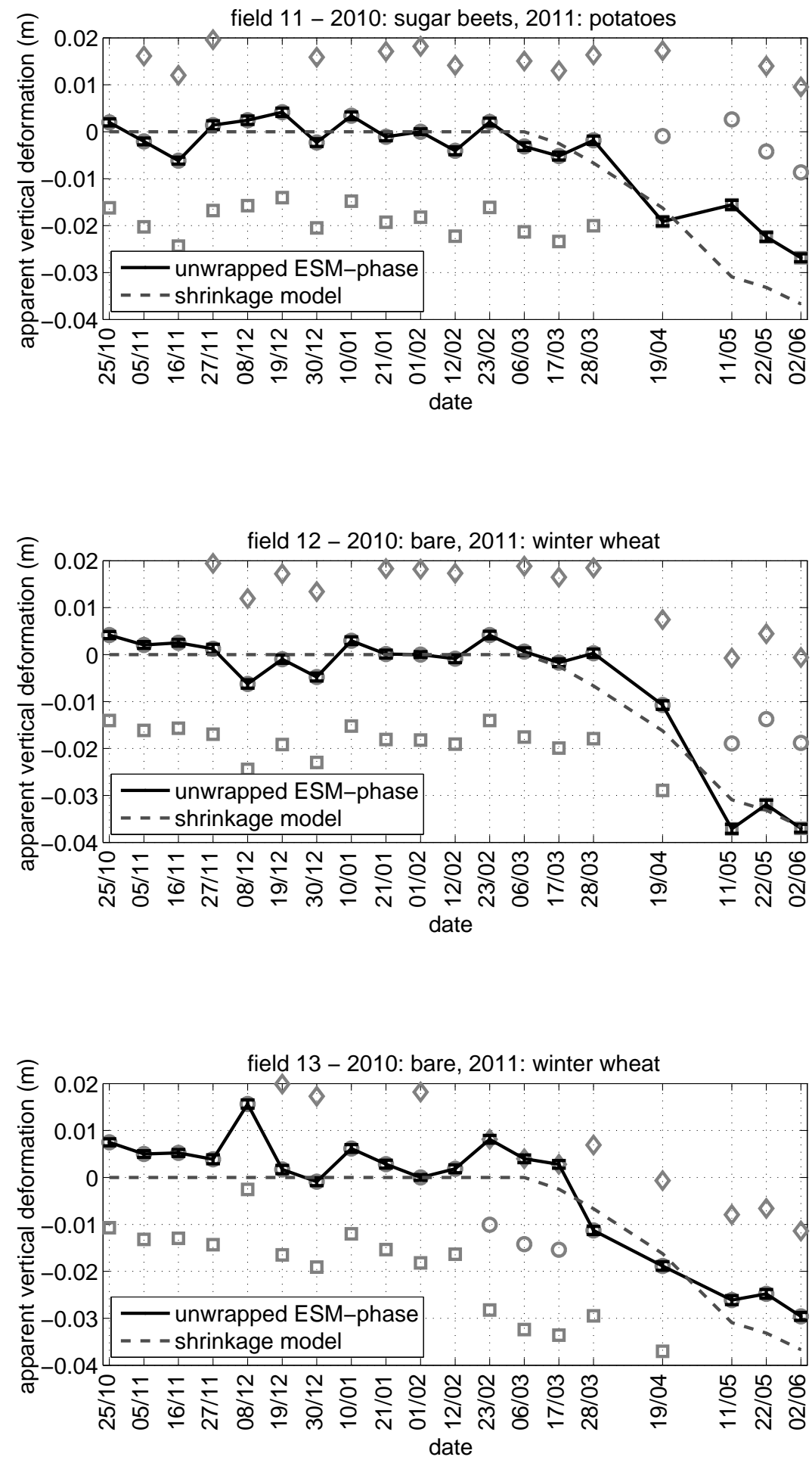

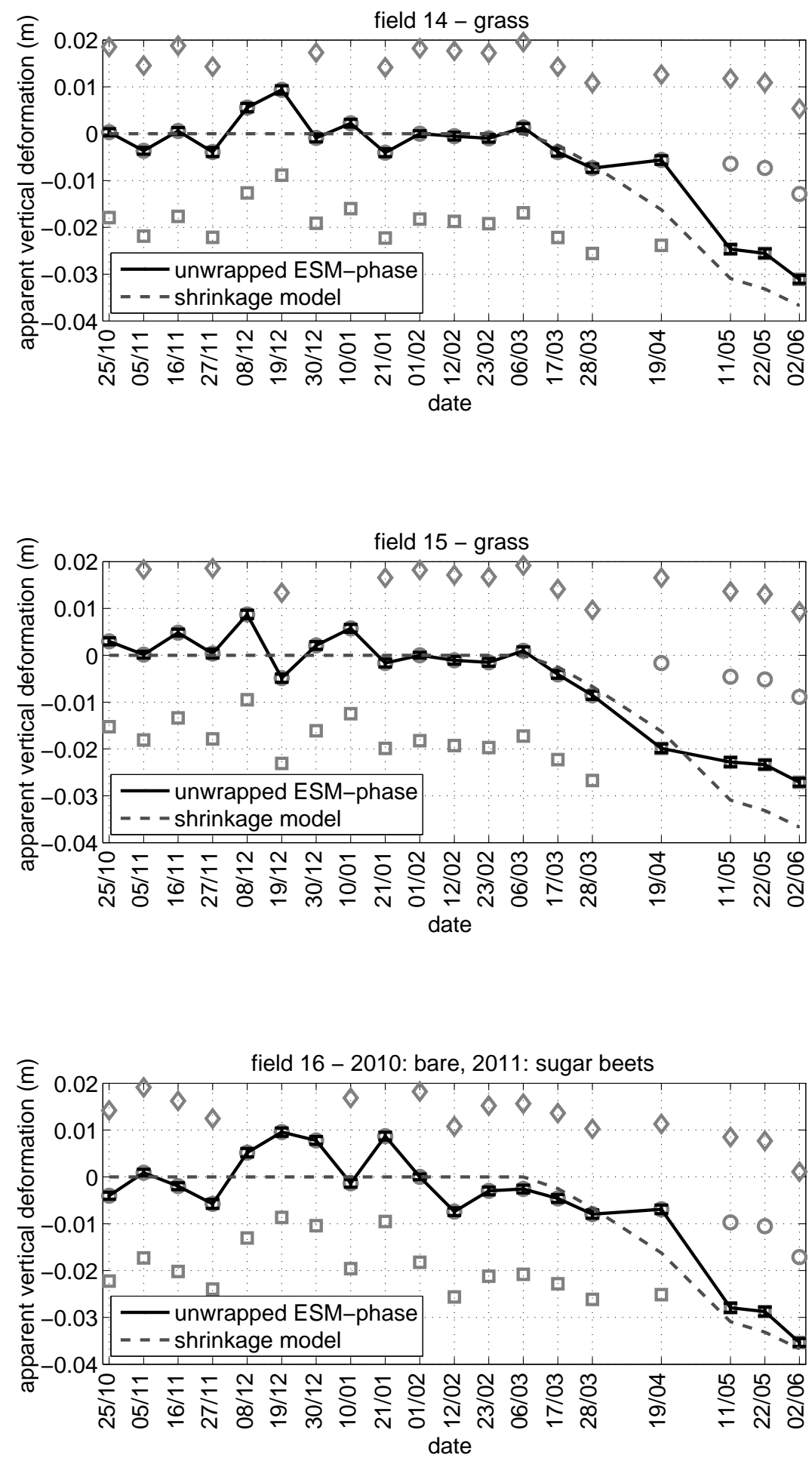

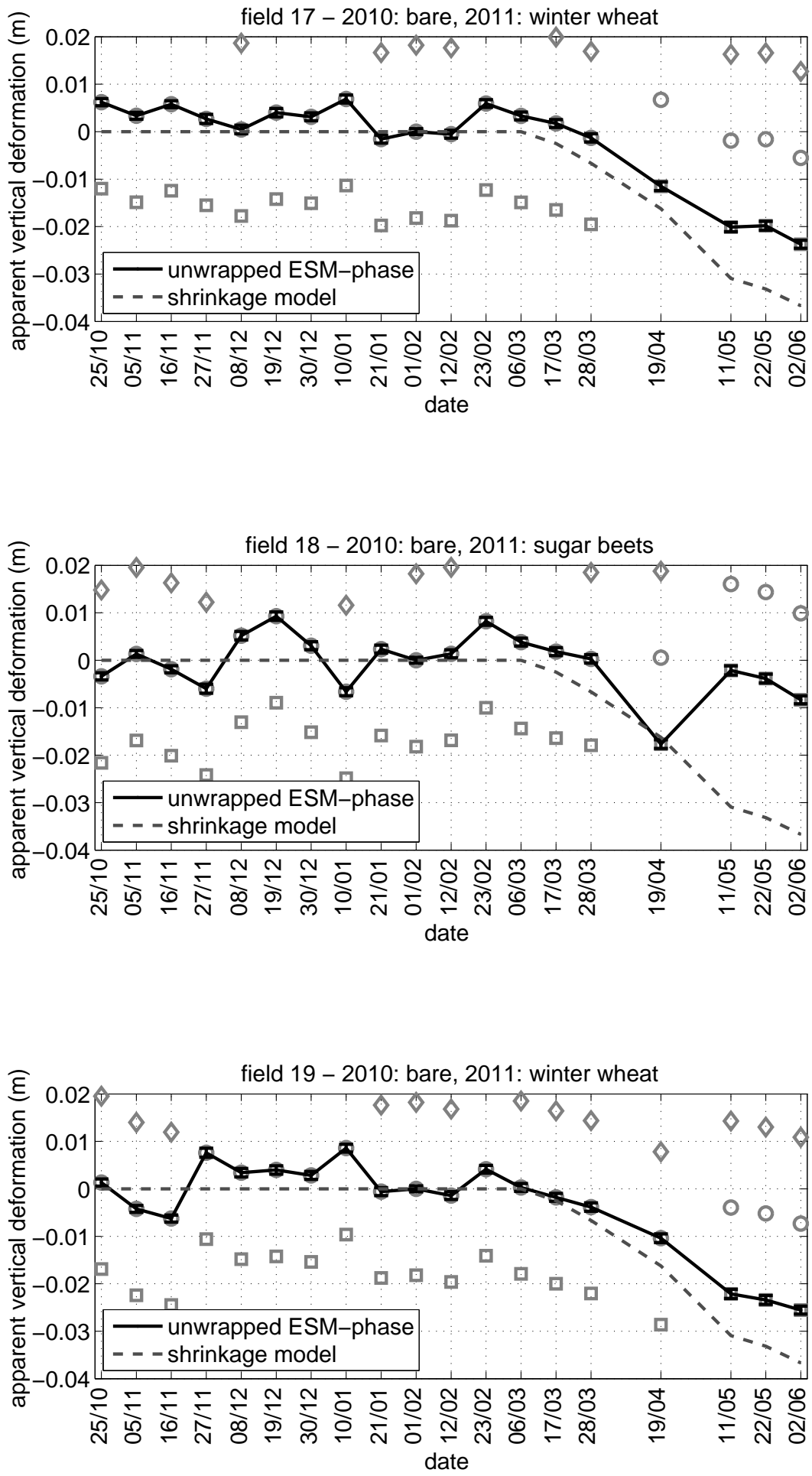

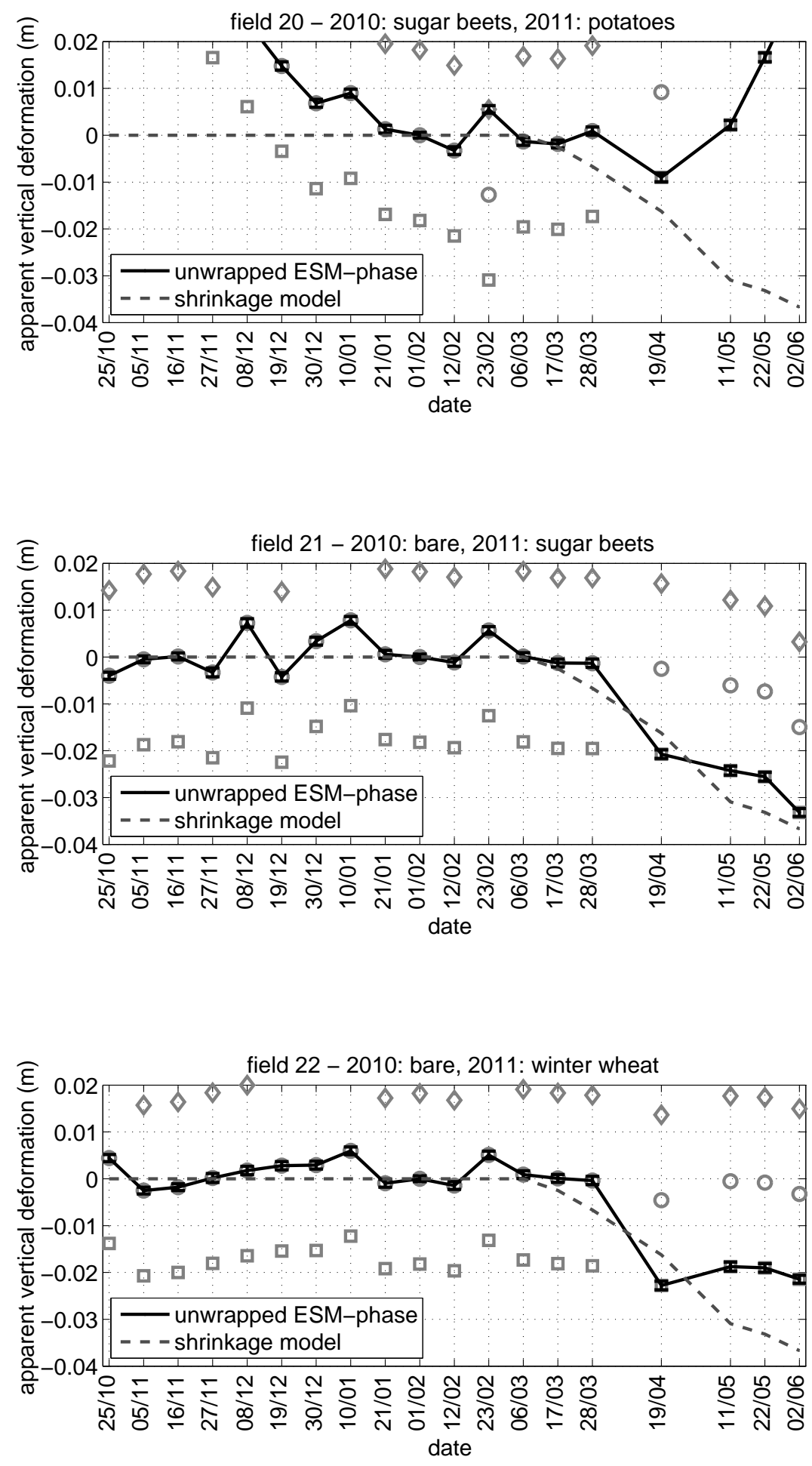

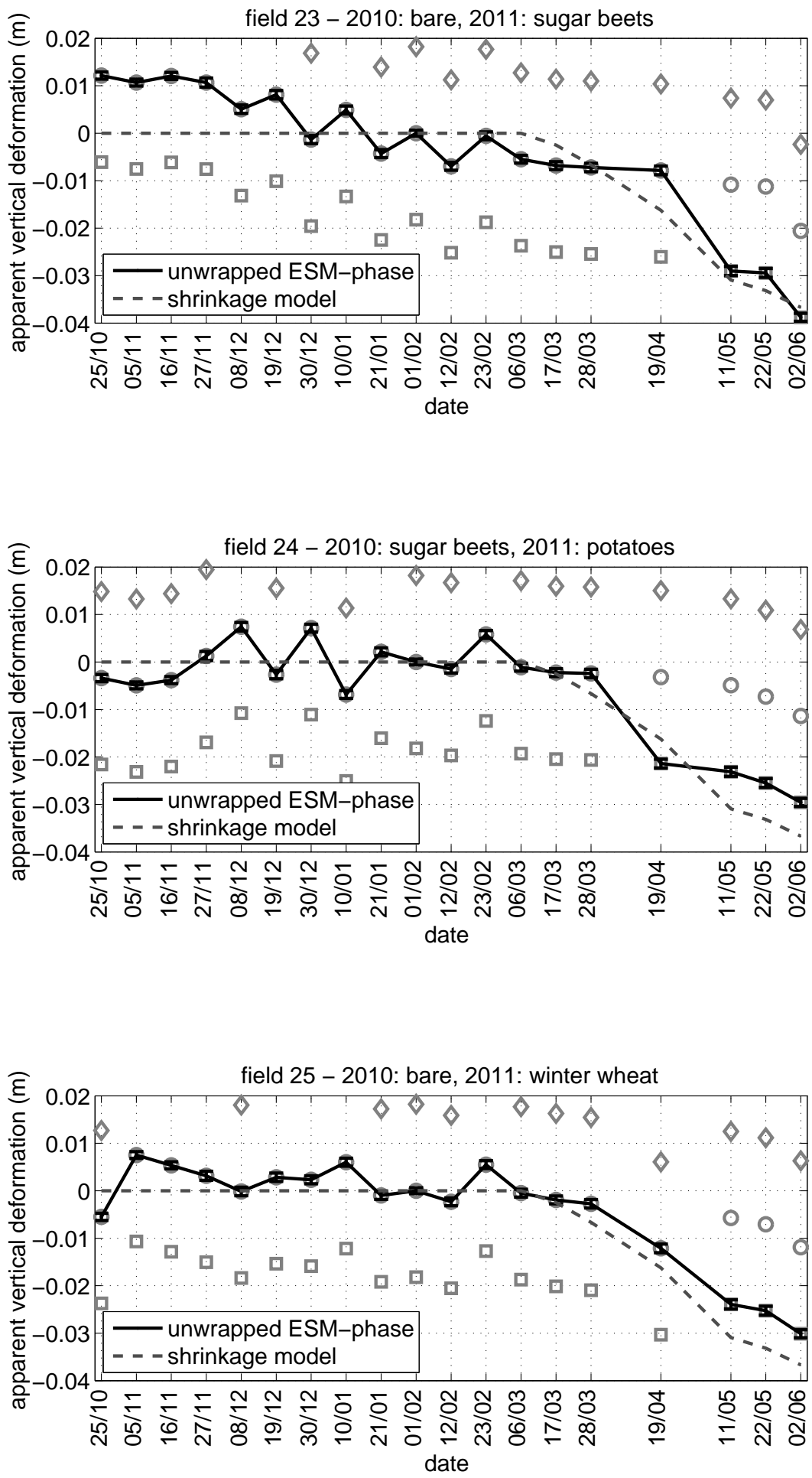


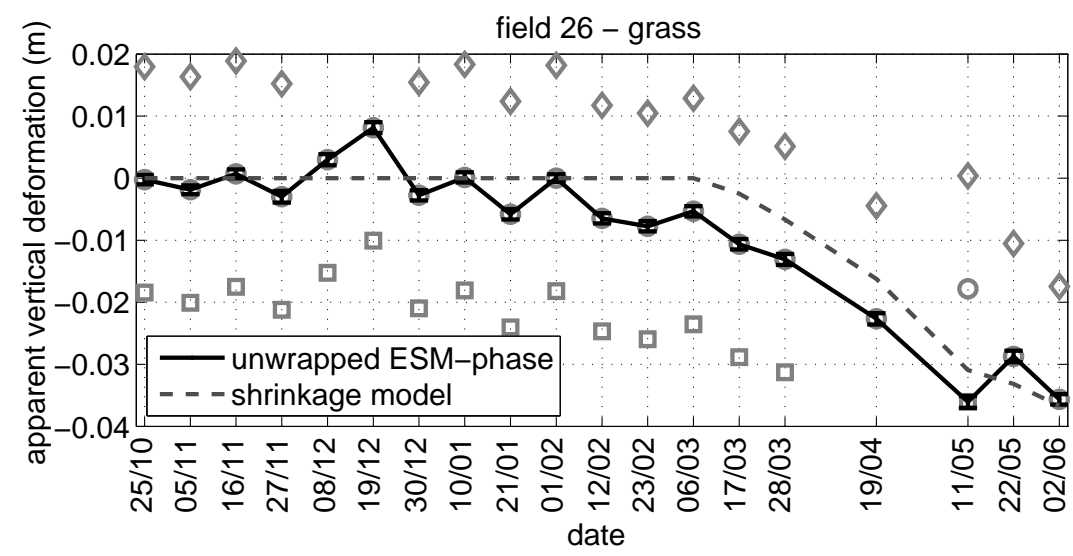

Fig. 6.9 Estimated deformation time series from ESM-phase corrected for dielectric phase and unwrapping errors, and modelled vertical shrinkage for all fields in the study area.

\subsection{Discussion and conclusion}

Clay swelling and shrinkage can be a significant phase contributor in InSAR time series and can lead to errors in the unwrapping of time series for X-band data. The shrinkage phase can be estimated using the proposed methodology, including improvement of the unwrapping performance based on a clay shrinkage model. Since the application of algorithms that use temporary coherent distributed scatterers over rural terrain is getting more common, assessment of clay shrinkage gets more relevant as well; either for shrinkage estimation for hydrological applications, or for the reduction of shrinkage phase for other InSAR applications. An a priori assessment of potential shrinkage and an estimation of its magnitude through soil maps and hydrometeorological data is valuable for correct interpretation of InSAR time series.

The proposed shrinkage model is useful for correction of the phase unwrapping, as it generally resolves the direction of deformation well, but the model overestimates vertical clay shrinkage compared to in situ measurements. The assumption of isotropic shrinkage still causes an underestimation of modelled vertical shrinkage, considering that in a more realistic case vertical shrinkage dominates horizontal shrinkage (as was found in Ch. 4). Exact quantification of shrinkage geometry is however hard and requires soil specific measurements, undesirable for a remotely sensed estimation of shrinkage.

The added value of the use of contextual data and models is shown here through application of an InSAR model for soil moisture based on in situ measured soil moisture content. Although the magnitude of the modelled soil moisture dielectric phase is small with respect to the clay shrinkage phase in this case study using X-band SAR data, the sensitivity to soil moisture dielectric phase could be much more pronounced in application of C-band or L-band data, or over non-clayey soil where no effect of shrinkage is present. It has to be mentioned that application of the soil moisture dielectric phase model is somewhat premature, as De Zan et al. (2014) indicated that 
further developments and testing are needed. The current study is best seen as an example of the combination of data to separate the phase from different sources, based on contextual data.

The methodology presented in this study offers a time series analysis technique to estimate clay shrinkage related to soil water storage change using X-band radar interferometry. Combining these measurement of vertical clay shrinkage with soil physical relations could lead to an approximation for soil water storage change in the vadose zone, as described in Ch. 4. To fully exploit this application, improved methods to quantify soil physical relations in clay soils are needed. Especially quantification of shrinkage geometry and volume change with water storage change in field soils is needed. Insights from Ch. 3 and 4 and the recently developed modelling framework by Stewart et al. (2016) contribute to this. 



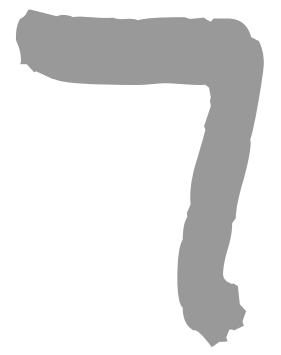

Synthesis

The main goal of this thesis was to develop the application of satellite based radar interferometry for estimation of soil water storage change on field scale to regional scale. In this chapter the most important findings from Ch. 3-6 are combined to evaluate to what extent the research objectives defined in Ch. 1 are met. Implications of the findings and directions for further research are discussed.

\subsection{Research objectives}

\subsubsection{Estimation of soil water storage change from surface elevation changes in clay soils}

The validity of the common assumptions of isotropic (e.g. Bronswijk, 1990; Arnold et al., 2005; Stewart et al., 2016) and normal shrinkage (Bronswijk \& Evers-Vermeer, 1990) have been tested for clay aggregates and field soils in Ch. 3 and Ch. 4 . If valid, these assumptions enable straightforward calculation of soil water storage changes from surface elevation changes: the water storage change equals volume change of the soil, which can be calculated from surface elevation change by modelling the soil as disconnected cubes (Bronswijk, 1990). Quantification of the relation between water content change and soil volume change, however, indicates a limited range of normal shrinkage for both aggregates and field soils, and a deviation from isotropic shrinkage for field soils. Calculation of water storage changes therefore requires the use of soil shrinkage characteristics of multiple layers in the soil and quantification of the shrinkage geometry factor $r_{\mathrm{s}}$ for field soils. 
In Ch. 3, volume and water content change of aggregates (the soil shrinkage curve, SSC) is quantified based on direct measurements on samples from soils with distinctive layering in terms of clay content. Maximum clay content was $23.6 \%$. It was found that the aggregates have moderate shrinkage potential over the soil moisture content range from saturation to air-dryness. Shrinkage phases were distinguished based on the portion of water content change that is compensated by volume change. About $40-50 \%$ of water was released in the normal shrinkage phase, where volume change fully compensates the loss of water. However, the residual shrinkage phase, where volume change is smaller than water content change, started at approximately $50 \%$ normalized soil moisture content (moisture content with respect to the moisture content at saturation). Normalized soil moisture content below $50 \%$ has been observed in the upper layers of a field soil (see Fig. 4.4), indicating the occurrence of residual and zero shrinkage in this situation.

In Ch. 4, the relation between water content change and soil volume change is quantified for field soils, based on in situ soil moisture content and surface elevation change measurements. Measurements were performed in 2010 and 2011. In 2011 progressive drying was observed with very limited re-wetting by precipitation; a situation comparable to drying conditions in the laboratory. In 2010 the drying regime was much more variable, with alternating drying and wetting of the soil. Unlike for the aggregates, normal shrinkage was not observed for the field soil, but rather a large degree of linear (basic) shrinkage was observed. Normal shrinkage is present over a large part of the relevant moisture range in aggregates (Ch. 3), but when drying out, different parts of a field soil do experience different shrinkage phases simultaneously. This results in less than normal shrinkage when the total unsaturated zone is considered. The water content change - volume change relation thus depends on the scale considered. The relation also depends on the drying regime, as the slope of the shrinkage curve was larger in 2011 than in 2010.

In the soil volume change calculations in Ch. 4, isotropic shrinkage was assumed. The comparison showed that assuming normal isotropic shrinkage does not yield a good approximation of soil water storage change. Assuming constant isotropic shrinkage (shrinkage geometry factor $r_{\mathrm{s}}=3$ ) resulted in slopes of the water content change - volume change relation larger than unity. To adjust for this, and to obtain a more realistic volume change calculation from vertical shrinkage measurements, $r_{\mathrm{s}^{-}}$ values were fitted to soil moisture content. This correction yielded $r_{\mathrm{s}}$-values between 1.38 and 3. Dynamics in subsidence porosity (i.e. vertical shrinkage) derived from the aggregate SSC and comparison with surface elevation change data from the field study also indicated $r_{\mathrm{s}}$-values considerably smaller than 3 (Ch. 3 ). These results show that vertical shrinkage (subsidence) is dominant over horizontal shrinkage (cracking) in the field soils studied.

In order to use soil surface elevation changes for estimating soil water storage change in clay soils, $r_{\mathrm{s}}$ variations with soil moisture content and the effect of variable drying and wetting conditions need to be considered. If the SSC is known and vertical shrinkage measurements can be obtained, these data can be used to estimate actual $r_{\mathrm{s}^{-}}$ values based on the model by Stewart et al. (2016), as applied in Ch. 3. Subsequently, the SSC can be applied to estimate soil water storage change, without assumptions 
on normal isotropic shrinkage.

\subsubsection{Measuring soil surface elevation changes from clay swelling and shrink- age on field to regional scale using satellite based radar interferome- try}

Two approaches have been applied in this thesis to isolate radar interferometric phase signals originating from surface elevation changes as a result of clay shrinkage: relative movement of two adjacent fields from interferograms with short temporal baseline (Ch. 5), and multi-interferogram time series analysis of individual fields. The analyses show that clay swelling and shrinkage can be a significant phase contributor in individual X-band SAR interferograms (Ch. 5) and in InSAR time series (Ch. 6), and that vertical clay shrinkage can be estimated from the phase observations.

In this thesis mainly the field scale has been considered. The spatial coverage (regional scale monitoring) and spatial observation density offered by SAR images, are not fully exploited. Instead, methodology development focused on a small area to enable comparison with in situ data and the high spatial resolution has been sacrificed on the benefit of noise reduction. The differential analysis in Ch. 5 indicates that considerable differences in vertical deformation (due to clay shrinkage or frost heave) can be expected between adjacent fields with similar soil properties and under similar weather conditions (precipitation, evaporation, near-surface temperature). In the analyses in this thesis, within field variation has been omitted by multilooking over all pixels within a field. Multilooking over all pixels might not be necessary to obtain good phase estimates, and the number of looks might be reduced for assessment of within field phase variation.

Elimination of unwanted phase contributions is key in interferometric analysis. The correction of unwanted phase contributions in Ch. 6 specifically included the soil moisture dielectric effect. Interferometric phases were predicted based on in situ measured soil moisture contents. The soil moisture dielectric phase was not found to be significant in X-band data, compared to the clay shrinkage phase.

Temporal phase unwrapping assuming a temporally smooth deformation signal proved to be inappropriate to reveal vertical shrinkage, based on the case study in Ch. 6. Therefore a simple model was developed to estimate vertical shrinkage, using assumption on shrinkage behaviour (normal and isotropic shrinkage) and an approximation of water storage change from precipitation and evapotranspiration data. The model was used to correct unwrapping results, thereby improving vertical shrinkage measurements from InSAR.

\subsubsection{Estimation of soil water storage change from clay shrinkage using satellite radar interferometry}

The results in this thesis, make clear that vertical clay shrinkage can be estimated from InSAR, using a model based on assumptions of normal and isotropic shrinkage. However, estimating soil water storage change from InSAR measured clay shrinkage needs quantification of the shrinkage geometry factor $r_{\mathrm{s}}$ and its dependence on drying regime and soil moisture content in a field soil. If vertical shrinkage and the soil shrinkage characteristic are known, subsidence porosity can be calculated to resolve 
the shrinkage geometry factor (Ch. 3). This however needs an estimation of the soil moisture content. The full estimation of soil water storage change from vertical shrinkage measured by InSAR, therefore needs an iterative procedure to account for soil moisture dependence of the shrinkage geometry factor.

\subsection{Outlook}

\subsubsection{Further development of the proposed methodology}

Deriving shrinkage geometry from in situ measurements is challenging, as it needs simultaneous monitoring of vertical and horizontal shrinkage. A practical, nondestructive, and non-labour intensive method to quantify the temporal changes in the volume of individual cracks has been developed by Stewart et al. (2012). Combining these measurements with soil layer thickness change measurements, as performed in Ch. 4 (or a more automated method, e.g. Neely et al., 2014), could deliver much needed insight in the timing and magnitude of crack development and closure with respect to vertical deformation, and thus the magnitude and dynamics of the shrinkage geometry factor in field soils. This would also involve a quantification of the field soil swelling process, which has been largely omitted in this thesis, due to measurement methodology limitations (e.g. difficulty of re-wetting of aggregates to measure the soil swelling curve, and temporal decorrelation of interferograms in periods with swelling). Quantification of both the swelling and shrinkage process with water content change would allow for assessment of how soil water storage change estimation is affected by hysteresis in these processes, on which no consensus exists in literature (Peng \& Horn, 2007; Chertkov, 2012b; Stewart et al., 2016, and Ch. 4). Considering the dependence of shrinkage geometry on drying regime, some steps have been taken by Peng et al. (2016), who quantified the effect of several wetting and drying cycles on estimation of soil cracking when using the shrinkage curve and geometry factor. Important to note here, is that these authors fitted geometry factors for young and old paddy fields, probably subject to structural changes (i.e. ripening) that affected the shrinkage geometry.

Increasing availability of data obtained by satellite missions with short repeat cycles, narrow orbital tubes, and C- and L-band SAR sensors, is expected to improve the capabilities of InSAR monitoring of clay shrinkage, as these factors contribute to limitation of temporal and geometrical decorrelation. Examples of such missions are the current Sentinel-1 mission, the upcoming RADARSAT Constellation (Canadian Space Agency, 2015), and the proposed Tandem-L mission (Moreira et al., 2015). However, the phase sensitivity to the soil moisture dielectric mechanism is expected to be higher for C-band or L-band radar compared to X-band radar. To quantify the interaction between soil moisture content and its vertical distribution (Rabus et al., 2010), dielectric properties of soils, and InSAR observations, dedicated measurements with laboratory set ups such as used by Nesti et al. (1998) and Morrison et al. (2011) are needed. If multiple frequency sensors are used simultaneous and experiments are performed on both shrinking and non-shrinking soils, these measurements will contribute to refine theory and models. Subsequently, analysis using satellite based data can be performed to test these models. Over agricultural terrain polarimetric 
SAR data could assist in decomposition of scattering mechanisms (e.g. Hajnsek \& Prats, 2008) over bare and vegetated agricultural terrain, to test the validity of (soil moisture) phase models.

Additional understanding of the phase contributions over rural terrain is needed for correct interpretation of the resulting phase time series and for phase reduction to isolate the contribution of interest. This includes accurate descriptions of how processing algorithms deal with certain contributions, such as soil moisture dielectric effects and volumetric scattering effects causing systematic phase inconsistencies (De Zan et al., 2014, 2015; Zwieback et al., 2016). To what extent phase effects from these mechanisms are filtered out from time series by ESM-phase estimation is still an open question, which requires more investigation.

The timing of clay shrinkage coincides largely with periods prone to temporal decorrelation (crop cover in summer). Despite expected progress in SAR data availability and processing algorithms, phase analysis over agricultural terrain will remain influenced by tillage activities, leading to temporal decorrelation and/or unexpected phase excursions. Knowledge of the field situation (e.g. soil type, land use, tillage activities) is therefore imperative in data selection, processing choices, and interpretation of phase time series, independent of the objective.

\subsection{Implications for hydrology}

InSAR can deliver vertical clay shrinkage measurement over large scales, relevant for regional water management, as from these measurements soil water storage change can be estimated. To refine soil water storage estimates, theories and models describing soil physical relations in clay soils are needed. The capability of InSAR to measure clay shrinkage could enhance the development of such models, as it allows regional scale observations that have been impossible before. This would require prolonged close cooperation between the soil science, hydrology, and InSAR communities. Such cooperation will not only be beneficial for soil water storage estimation, but could also boost new applications and deliver detailed information on phase governing processes over rural and natural terrain.

For the specific application of estimating soil water storage change, the advantage of the use of InSAR is twofold: i) observation are done over regional scale with fine spatial resolution, and ii) the cumulative effect of all water storage change above the groundwater table, adds to the measurable effect of vertical shrinkage on the soil surface. For water balance studies, the latter is a benefit of InSAR over remote sensing techniques for soil moisture content estimation, based on variables defined by near-surface conditions (i.e. the upper few centimetres of the soil). From this perspective, InSAR estimates of clay dynamics are more valuable than InSAR estimates of the dielectric soil moisture effect. Additionally, using X-band SAR over clay soils, there seems to be more potential for water storage estimation from geometric phase contributions resulting from vertical clay shrinkage, compared to dielectric phase contributions, as the geometric phase signal is larger and there is more sensitivity of phase to vertical shrinkage than to dielectric changes.

Independent of the remaining challenge for soil water storage estimation, the InSAR 
measurements allow for the identification of periods and areas with large shrinkage, in which shrinkage cracks are likely to occur. These cracks enable rapid transport of nutrients and pollutants to groundwater and surface water (Jarvis \& Leeds-Harrison, 1990; Wells et al., 2003). Especially the regional scale observations are valuable, as spatial patterns in shrinkage, and thus in risks of groundwater pollution can be assessed. This far, such observation based risk analysis have been impossible, unless a very dense network of in situ measurement would be deployed.

\subsection{Implications for radar interferometry}

Clay shrinkage can cause unwrapping errors if not accounted for. Also if clay shrinkage is not the mechanism of interest, it should be considered in corrections of interferometric phase. This is especially important with increasing possibilities to use temporary coherent distributed scatterers for InSAR phase analysis.

In this thesis, vertical clay shrinkage was observed with InSAR applied over an area with limited clay content, limited clay shrinkage potential, and limited depth of the unsaturated zone. Extraordinary dry conditions for Dutch circumstances however, made a very good case study in 2011 . When we realize there are areas in the world with soils with high shrinkage potential, deep unsaturated zones, and where prolonged drying (like in the Dutch 2011 spring) is standard rather than extraordinary, phase change due to shrinkage is expected to be a regular phenomenon, potentiality affecting many interferograms over these areas. 


\section{Bibliography}

Aitchison, G. D., \& Holmes, J. W. 1953. Aspects of swelling in the soil profile. Aust. J. App. Sci., 4, 244-259.

Alsdorf, D., Birkett, C., Dunne, T., Melack, J., \& Hess, L. 2001. Water level changes in a large Amazon lake measured with spaceborne radar interferometry and altimetry. Geophys. Res. Lett., 28, 2671-2674.

Alshawaf, F., Fersch, B., Hinz, S., Kunstmann, H., Mayer, M., \& Meyer, F. J. 2015. Water vapor mapping by fusing InSAR and GNSS remote sensing data and atmospheric simulations. Hydrol. Earth Syst. Sci., 19, 4747-4764.

Alterra. 2006. Grondsoortenkaart.lyr, ArcGis layer file. Last accessed 10 Dec. 2012.

Amelung, F., Galloway, D. L., Bell, J. W., Zebker, H. A., \& Laczniak, R. J. 1999. Sensing the ups and downs of Las Vegas: InSAR reveals structural control of land subsidence and aquifer-system deformation. Geology, 27(Jun), 483-486.

Arnold, J. G., Potter, K. N., King, K. W., \& Allen, P. M. 2005. Estimation of soil cracking and the effect on surface runoff in a Texas Blackland Prairie watershed. Hydrol. Process., 19.

Arya, L. M., Richter, J. C., \& Paris, J. F. 1983. Estimating profile water storage from surface zone soil-moisture measurements under bare field conditions. Water Resour. Res., 19, 403-412.

Bamler, R., \& Hartl, P. 1998. Synthetic aperture radar interferometry. Inverse Probl., 14(Aug), R1-R54.

Ben Khadhra, K., Nolan, M., Börner, T., Hounam, D., \& Chandra, M. 2006. Phase sensitivity to soil moisture in controlled anechoic chamber: Measurements and first results. In: German Microwave Conference-GeMiC, vol. 2006.

Berardino, P., Fornaro, G., Lanari, R., \& Sansosti, E. 2002. A New Algorithm for Surface Deformation Monitoring Based on Small Baseline Differential SAR Interferograms. IEEE Trans. Geosci. Remote Sens., 40, 2375-2383.

Bhattacharyya, S., Tripp, A. C., \& McNearnyd, R. L. 2007. Soil moisture effects on temporal subsidence estimates using InSAR. Int. J. Min. Reclam. Env., 21, 88-96.

Blöschl, G., \& Sivapalan, M. 1995. Scale issues in hydrological modelling: a review. Hydrol. Process., 9, 251-290.

Bogena, H. R., Huisman, J. A., Oberdoerster, C., \& Vereecken, H. 2007. Evaluation of a low-cost soil water content sensor for wireless network applications. J. Hydrol., 344, 32-42.

Bogena, H. R., Huisman, J. A., Meier, H., Rosenbaum, U., \& Weuthen, A. 2009. Hy- 
brid Wireless Underground Sensor Networks: Quantification of Signal Attenuation in Soil. Vadose Zone J., 8, 755-761.

Bogena, H. R., Herbst, M., Huisman, J. A., Rosenbaum, U., Weuthen, A., \& Vereecken, H. 2010. Potential of Wireless Sensor Networks for Measuring Soil Water Content Variability. Vadose Zone J., 9, 1002-1013.

Boivin, P. 2007. Anisotropy, cracking, and shrinkage of vertisol samples: Experimental study and shrinkage modeling. Geoderma, 138, 25-38.

Bonì, R., Cigna, F., Bricker, S., Meisina, C., \& McCormack, H. 2016a. Characterisation of hydraulic head changes and aquifer properties in the London Basin using Persistent Scatterer Interferometry ground motion data. J. Hydrol., 540, 835-849.

Bonì, R., Pilla, G., \& Meisina, C. 2016b. Methodology for Detection and Interpretation of Ground Motion Areas with the A-DInSAR Time Series Analysis. Remote Sens., 8.

Brasher, B. R., Franzmeier, D. P., Valassis, V., \& Davidson, S. E. 1966. Use Of Saran Resin To Coat Natural Soil Clods For Bulk-Density And Water-Retention Measurements. Soil Sci., 101.

Braudeau, E., \& Mohtar, R. H. 2006. Modeling the swelling curve for packed soil aggregates using the pedostructure concept. Soil Sci. Soc. Am. J., 70, 494-502.

Braudeau, E., Costantini, J. M., Bellier, G., \& Colleuille, H. 1999. New device and method for soil shrinkage curve measurement and characterization. Soil Sci. Soc. Am. J., 63, 525-535.

Braudeau, E., Frangi, J. P., \& Mohtar, R. H. 2004. Characterizing nonrigid aggregated soil-water medium using its shrinkage curve. Soil Sci. Soc. Am. J., 68, 359-370.

Bridge, B. J., \& Ross, P. J. 1984. Relations among physical properties of cracking clay soils. In: J. W. McGarity, E. H. Hoult, \& So, H. B. (eds), Properties and Utilisation of Cracking Clay Soils. University of New England: Armidale, New South Wales, Australia.

Bronstert, A., Creutzfeldt, B., Graeff, T., Hajnsek, I., Heistermann, M., Itzerott, S., Jagdhuber, T., Kneis, D., Luck, E., Reusser, D., \& Zehe, E. 2012. Potentials and constraints of different types of soil moisture observations for flood simulations in headwater catchments. Nat. Hazards, 60, 879-914.

Bronswijk, J. J. B. 1988. Modeling of water balance, cracking and subsidence of clay soils. J. Hydrol., 97, 199-212.

Bronswijk, J. J. B. 1990. Shrinkage geometry of a heavy clay soil at various stresses. Soil Sci. Soc. Am. J., 54, 1500-1502.

Bronswijk, J. J. B. 1991a. Drying, cracking, and subsidence of a clay soil in a lysimeter. Soil Sci., 152, 92-99.

Bronswijk, J. J. B. 1991b. Relation between vertical soil movements and water-content changes in cracking clays. Soil Sci. Soc. Am. J., 55, 1220-1226.

Bronswijk, J. J. B., \& Evers-Vermeer, J. J. 1990. Shrinkage of Dutch clay soil aggregates. Neth. J. Agr. Sci., 38, 175-194.

Brown, K. F., Messem, A. B., Dunham, R. J., \& Biscoe, P. V. 1987. Effect of drought on growth and water-use of sugar-beet. J. Agr. Sci., 109, 421-435. 
Buurman, P., Pape, Th., Reijneveld, J. A., De Jong, F., \& Van Gelder, E. 2001. Laser-diffraction and pipette-method grain sizing of Dutch sediments: correlations for fine fractions of marine, fluvial, and loess samples. Neth. J. Geosci., 80.

Cabidoche, Y. M., \& Ozier-Lafontaine, H. O. 1995. Theresa 1: Matric water-content measurements through-thickness variations in vertisols. Agr. Water Manage., 28, $133-147$.

Cabidoche, Y. M., \& Voltz, M. 1995. Nonuniform volume and water-content changes in swelling clay soil. 2: A field-study on a vertisol. Eur. J. Soil Sci., 46, 345-355.

Campbell Scientific, Inc. 2006. CS616 and CS625 Water Content Reflectometers, Instruction manual. Tech. rept.

Camposeo, S, \& Rubino, P. 2003. Effect of irrigation frequency on root water uptake in sugar beet. Plant Soil, 253, 301-309.

Canadian Space Agency. 2015. http://www.asc-csa.gc.ca. Last accessed: 22 January 2017.

Cardell-Oliver, R, Kranz, M, Smettem, K, \& Mayer, K. 2005. A reactive soil moisture sensor network: Design and field evaluation. Int. J. Distrib. Sens. N., 1, 149-162.

Caro Cuenca, M. 2012. Improving radar interferometry for monitoring fault-related surface deformation. Ph.D. thesis, Delft University of Technology.

Chang, L., \& Hanssen, R. F. 2015. Detection of permafrost sensitivity of the QinghaiTibet railway using satellite radar interferometry. Int. J. Remote Sens., 36, 691-700.

Chang, L., \& Hanssen, R. F. 2016. A Probabilistic Approach for InSAR Time Series Postprocessing. IEEE Trans. Geosci. Remote Sens., 54(1), 421-430.

Chen, J., Knight, R., Zebker, H. A., \& Schreüder, W. A. 2016. Confined aquifer head measurements and storage properties in the San Luis Valley, Colorado, from spaceborne InSAR observations. Water Resour. Res., 52(5), 3623-3636.

Chertkov, V. Y. 2005. The Shrinkage Geometry Factor of a Soil Layer. Soil Sci. Soc. Am. J., 69, 1671-1683.

Chertkov, V. Y. 2007a. The reference shrinkage curve at higher than critical soil clay content. Soil Sci. Soc. Am. J., 71, 641-655.

Chertkov, V. Y. 2007b. The soil reference shrinkage curve. Open Hydrol. J., 1, 1-18.

Chertkov, V. Y. 2008. The geometry of soil crack networks. Open Hydrol. J., 2, 34-48.

Chertkov, V. Y. 2012a. An integrated approach to soil structure, shrinkage, and cracking in samples and layers. Geoderma, 173, 258-273.

Chertkov, V. Y. 2012b. Physical modeling of the soil swelling curve vs. the shrinkage curve. Adv. Water Resour., 44, 66-84.

Chertkov, V. Y., Ravina, I., \& Zadoenko, V. 2004. An approach for estimating the shrinkage geometry factor at a moisture content. Soil Sci. Soc. Am. J., 68, 18071817 .

Coquet, Y., Touma, J., \& Boivin, P. 1998. Comparison of soil linear shrinkage curve from extracted cores and in situ. Aust. J. Soil Res., 36, 765-782.

Cornelis, W. M., Corluy, J., Medina, H., Díaz, J., Hartmann, R., Van Meirvenne, M., \& Ruiz, M. E. 2006. Measuring and modelling the soil shrinkage characteristic 
curve. Geoderma, 137, 179-191.

Corti, T., Muccione, V., Köllner-Heck, P., Bresch, D., \& Seneviratne, S. I. 2009. Simulating past droughts and associated building damages in France. Hydrol. Earth Syst. Sci., 13.

Corti, T., Wüest, M., Bresch, D., \& Seneviratne, S. I. 2011. Drought-induced building damages from simulations at regional scale. Nat. Hazard Earth Syst., 11.

Creutzfeldt, B., Guntner, A., Vorogushyn, S., \& Merz, B. 2010. The benefits of gravimeter observations for modelling water storage changes at the field scale. $H y$ drol. Earth Syst. Sci., 14, 1715-1730.

Crow, W. T., \& Ryu, D. 2009. A new data assimilation approach for improving runoff prediction using remotely-sensed soil moisture retrievals. Hydrol. Earth Syst. Sci., 13, $1-16$.

Curlander, J. C., \& MacDonough, R. N. 1991. Synthetic aperture radar: systems and signal processing. Wiley series in remote sensing. New York: Wiley.

Dagesse, D. F. 2010. Freezing-induced bulk soil volume changes. Can. J. Soil Sci., 90, 389-401.

De Bruin, H. A. R. 1987. From Penman to Makkink. In: Hooghart, J. C. (ed), Evaporation and Weather. Proceedings and information No. 39. Comm. Hydrol. Res. TNO.

De Bruin, H. A. R., \& Lablans, W. N. 1998. Reference crop evapotranspiration determined with a modified Makkink equation. Hydrol. Process., 12, 1053-1062.

De Vries, J. J., \& Simmers, I. 2002. Groundwater recharge: an overview of processes and challenges. Hydrogeology J., 10, 5-17.

De Zan, F., Parizzi, A., Prats-Iraola, P., \& López-Dekker, P. 2014. A SAR Interferometric Model for Soil Moisture. IEEE Trans. Geosci. Remote Sens., 52, 418-425.

De Zan, F., Zonno, M., \& López-Dekker, P. 2015. Phase Inconsistencies and Multiple Scattering in SAR Interferometry. IEEE Trans. Geosci. Remote Sens., 53, 66086616.

Decagon Devices, Inc. 2010. EC-20, EC-10, EC-5, Soil Moisture Sensors User's Manual. Tech. rept.

Diao, F., Walter, T. R., Motagh, M., Prats-Iraola, P., Wang, R., \& Samsonov, S. V. 2015. The 2015 Gorkha earthquake investigated from radar satellites: slip and stress modeling along the MHT. Front. Earth Sci., 3.

Draper, C. S., Reichle, R. H., De Lannoy, G. J. M., \& Liu, Q. 2012. Assimilation of passive and active microwave soil moisture retrievals. Geophys. Res. Lett., 39.

Dubois, P. C., Van Zyl, J., \& Engman, T. 1995. Measuring soil moisture with imaging radars. IEEE Trans. Geosci. Remote Sens., 33, 915-926.

Eagleson, P. S. 1978a. Climate, soil, and vegetation: 1. Introduction to water balance dynamics. Water Resour. Res., 14, 705-712.

Eagleson, P. S. 1978b. Climate, soil, and vegetation. 6. Dynamics of the Annual Water Balance. Water Resour. Res., 14, 749-764.

Edwards, L. M., \& Burney, J. R. 1989. The effect of antecedent freeze-thaw frequency on runoff and soil loss from frozen soil with and without subsoil compaction and 
ground cover. Can. J. Soil Sci., 69, 799-811.

FAO/IIASA/ISRIC/ISSCAS/JRC. 2012. Harmonized World Soil Database (version 1.2). FAO, Rome, Italy and IIASA, Laxenburg, Austria.

Farmer, D., Sivapalan, M., \& Jothityangkoon, C. 2003. Climate, soil, and vegetation controls upon the variability of water balance in temperate and semiarid landscapes: Downward approach to water balance analysis. Water Resour. Res., 39.

Feddes, R. 1987. Crop factors in relation to Makkink's reference crop evapotranspiration. In: Hooghart, J. C. (ed), Evaporation and Weather. Proceedings and information No. 39. Comm. Hydrol. Res. TNO.

Ferretti, A., Prati, C., \& Rocca, F. 2000. Nonlinear subsidence rate estimation using permanent scatterers in differential SAR interferometry. IEEE Trans. Geosci. Remote Sens., 38, 2202-2212.

Ferretti, A., Prati, C., \& Rocca, F. 2001. Permanent scatterers in SAR interferometry. IEEE Trans. Geosci. Remote Sens., 39, 8-20.

Ferretti, A., Fumagalli, A., Novali, F., Prati, C., Rocca, F., \& Rucci, A. 2011. A New Algorithm for Processing Interferometric Data-Stacks: SqueeSAR. IEEE Trans. Geosci. Remote Sens., 49, 3460-3470.

Foster, J., Brooks, B., Cherubini, T., Shacat, C., Businger, S., \& Werner, C. L. 2006. Mitigating atmospheric noise for InSAR using a high resolution weather model. Geophys. Res. Lett., 33.

Francesca, V., Osvaldo, F., Stefano, P., \& Paola, R. P. 2010. Soil Moisture Measurements: Comparison of Instrumentation Performances. J. Irrig. Drain. E-ASCE, 136, 81-89.

Gabriel, A. K., Goldstein, R. M., \& Zebker, H. A. 1989. Mapping Small Elevation Changes Over Large Areas: Differential Radar Interferometry. J. Geophys. Res., 94.

Galloway, D. L., Hudnut, K. W., Ingebritsen, S. E., Phillips, S. P., Peltzer, G., Rogez, F., \& Rosen, P. A. 1998. Detection of aquifer system compaction and land subsidence using interferometric synthetic aperture radar, Antelope Valley, Mojave Desert, California. Water Resour. Res., 34, 2573-2585.

Gao, J. 2007. Towards accurate determination of surface height using modern geoinformattic methods: possibilities and limitations. Prog. Phys. Geog., 31, 591-605.

Gatelli, F., Guarnieri, A. M., Parizzi, F., Pasquali, P., Prati, C., \& Rocca, F. 1994. The wave-number shift in sar interferometry. IEEE Trans. Geosci. Remote Sens., 32, 855-865.

Gee, G. W., \& Hillel, D. 1988. Groundwater recharge in arid regions - Review and critique of estimation methods. Hydrol. Process., 2, 255-266.

Gens, R. 2003. Two-dimensional phase unwrapping for radar interferometry: Developments and new challenges. Int. J. Remote Sens., 24, 703-710.

Gens, R., \& Van Genderen, J. L. 1996. SAR interferometry - Issues, techniques, applications. Int. J. Remote Sens., 17, 1803-1835.

Goel, K., \& Adam, N. 2014. A distributed scatterer interferometry approach for precision monitoring of known surface deformation phenomena. IEEE Trans. Geosci. 
Remote Sens., 52, 5454-5468.

Goldstein, R. 1995. Atmospheric limitations to repeat-track radar interferometry. Geophys. Res. Lett., 22, 2517-2520.

Goldstein, R. M., Zebker, H. A., \& Werner, C. L. 1988. Satellite radar interferometry: Two-dimensional phase unwrapping. Radio Sci., 23, 713-720.

Gong, W., Meyer, F. J., Lee, C.-W., Lu, Z., \& Freymueller, J. 2015a. Measurement and interpretation of subtle deformation signals at Unimak Island from 2003 to 2010 using weather model-assisted time series InSAR. J. Geophys. Res.-Sol. Ea., 120, 1175-1194.

Gong, W., Meyer, F.J., Liu, S., \& Hanssen, R.F. 2015b. Temporal Filtering of InSAR Data Using Statistical Parameters From NWP Models. IEEE Trans. Geosci. Remote Sens., 1-12.

Groenevelt, P. H., \& Grant, C. D. 2001. Re-evaluation of the structural properties of some British swelling soils. Eur. J. Soil Sci., 52, 469-477.

Grossman, R. B., Brasher, B. R., Franzmeier, D. P., \& Walker, J. L. 1968. Linear Extensibility as Calculated from Natural-Clod Bulk Density Measurements. Soil Sci. Soc. Am. J., 32, 570-573.

Haines, W. B. 1923. The volume-changes associated with variations of water content in soil. J. Agr. Sci., 13, 296-310.

Hajnsek, I., \& Prats, P. 2008. Soil Moisture Estimation in time with D-InSAR. In: IGARSS 2008 - 2008 IEEE International Geoscience and Remote Sensing Symposium.

Hallikainen, M. T., Ulaby, F. T., Dobson, M. C., El-rayes, M. A., \& Wu, L. K. 1985. Microwave Dielectric Behavior of Wet Soil-Part 1: Empirical Models and Experimental Observations. IEEE Trans. Geosci. Remote Sens., 25-34.

Hanssen, R. F. 2001. Radar interferometry: data interpretation and error analysis. Remote sensing and digital image processing. Dordrecht, the Netherlands: Kluwer Academic Publishers.

Hanssen, R. F., \& Klees, R. 1999. An empirical model for the assessment of DEM accuracy degredation due to atmospheric stratification. In: Proc. FRINGE 1999 Workshop.

Hanssen, R. F., Teunissen, P. J. G., \& Joosten, P. 2001. Phase ambiguity resolution for stacked radar interferometric data. In: Proc KIS2001, international symposium on kinematic systems in geodesy, Geomatics and Navigation, Banff.

Hazeu, G. W. 2005. Landelijk Grondgebruiksbestand Nederland (LGN5). Vervaardiging, nauwkeurigheid en gebruik. Alterra-report 1213. Wageningen: Alterra.

Hong, S. H., Wdowinski, S., \& Kim, S. W. 2010. Evaluation of TerraSAR-X Observations for Wetland InSAR Application. IEEE Trans. Geosci. Remote Sens., 48, 864-873.

Hooper, A. 2008. A multi-temporal InSAR method incorporating both persistent scatterer and small baseline approaches. Geophys. Res. Lett., 35.

Hooper, A., Zebker, H., Segall, P., \& Kampes, B. 2004. A new method for measuring deformation on volcanoes and other natural terrains using InSAR persistent 
scatterers. Geophys. Res. Lett., 31.

Huisman, J. A., Hubbard, S. S., Redman, J. D., \& Annan, A. P. 2003. Measuring Soil Water Content with Ground Penetrating Radar: A Review. Vadose Zone J., 2, 476-491.

Jackson, T. J. 2002. Remote sensing of soil moisture: implications for groundwater recharge. Hydrogeology J., 10, 40-51.

Jarvis, N. J., \& Leeds-Harrison, P. B. 1990. Field test of a water balance model of cracking clay soils. J. Hydrol., 112, 203-218.

Jayawardane, N. S., \& Greacen, E. L. 1987. The nature of swelling in soils. Aust. J. Soil Res., 25, 107-113.

Jones, L. D., \& Terrington, R. 2011. Modelling Volume Change Potential in the London Clay. Q. J. Eng. Geol. Hydroge., 44.

Kampes, B. M., \& Hanssen, R. F. 2004. Ambiguity resolution for permanent scatterer interferometry. IEEE Trans. Geosci. Remote Sens., 42, 2446-2453.

Kennedy, G. W., \& Price, JS. 2005. A conceptual model of volume-change controls on the hydrology of cutover peats. J. Hydrol., 302.

Ketelaar, V. B. H. 2009. Subsidence Due to Hydrocarbon Production in the Netherlands. Springer, Netherlands.

Kirby, J. M., Bernardi, A. L., Ringrose-Voase, A. J., Young, R., \& Rose, H. 2003. Field swelling, shrinking, and water content change in a heavy clay soil. Aust. J. Soil Res., 41, 963-978.

Klees, R., \& Massonnet, D. 1998. Deformation measurements using SAR interferometry: potential and limitations. Neth. J. Geosci., 77.

KNMI. 2014. http://www.knmi.nl. Last accessed: 23 July 2014.

Kumar, S. V., Reichle, R. H., Koster, R. D., Crow, W. T., \& Peters-Lidard, C. D. 2009. Role of Subsurface Physics in the Assimilation of Surface Soil Moisture Observations. J. Hydrometeorol., 10, 1534-1547.

Lievens, H., Verhoest, N. E. C., De Keyser, E., Vernieuwe, H., Matgen, P., ÁlvarezMozos, J., \& De Baets, B. 2011. Effective roughness modelling as a tool for soil moisture retrieval from C- and L-band SAR. Hydrol. Earth Syst. Sci., 15, 151-162.

Liu, Q., Reichle, R. H., Bindlish, R., Cosh, M. H., Crow, W. T., De Jeu, R., De Lannoy, G. J. M., Huffman, G. J., \& Jackson, T. J. 2011. The Contributions of Precipitation and Soil Moisture Observations to the Skill of Soil Moisture Estimates in a Land Data Assimilation System. J. Meteorol, 12, 750-765.

Liu, S., Mika, A., Gong, W., Hanssen, R. F., Meyer, F., Morton, D., \& Webley, P. W. 2011. The role of weather models in mitigation of tropospheric delay for SAR interferometry. In: Geoscience and Remote Sensing Symposium (IGARSS), 2011.

Logsdon, S. D. 2009. CS616 Calibration: Field versus Laboratory. Soil Sci. Soc. Am. $J ., 73,1-6$.

Lu, Z., \& Kwoun, O. I. 2008. Radarsat-1 and ERS InSAR analysis over southeastern coastal Louisiana: Implications for mapping water-level changes beneath swamp forests. IEEE Trans. Geosci. Remote Sens., 46, 2167-2184.

Mahapatra, P. S., Samiei-Esfahany, S., der Marel, H. Van, \& Hanssen, R. F. 2014. 
On the Use of Transponders as Coherent Radar Targets for SAR Interferometry. IEEE Trans. Geosci. Remote Sens., 52, 1869-1878.

Marinkovic, P. S., Ketelaar, V. B. H., Van Leijen, F. J., \& Hanssen, R. F. 2008. InSAR quality control: analysis of five years of corner reflector time series. In: Proc. FRINGE 2007 Workshop.

Massonnet, D., \& Feigl, K. L. 1998. Radar interferometry and its application to changes in the earth's surface. Rev. Geophys., 36, 441-500.

Massonnet, D., Briole, P., \& Arnaud, A. 1995. Deflation of Mount Etna monitored by spaceborne radar interferometry. Nature, 375, 567-570.

Massonnet, D., Holzer, T., \& Vadon, H. 1997. Land subsidence caused by the East Mesa geothermal field, California, observed using SAR interferometry. Geophys. Res. Lett., 24, 901-904.

Mattia, F., Satalino, G., Pauwels, V. R. N., \& Loew, A. 2009. Soil moisture retrieval through a merging of multi-temporal L-band SAR data and hydrologic modelling. Hydrol. Earth Syst. Sci., 13, 343-356.

Milillo, P., Bürgmann, R., Lundgren, P., Salzer, J., Perissin, D., Fielding, E., Biondi, F., \& Milillo, G. 2016. Space geodetic monitoring of engineered structures: The ongoing destabilization of the Mosul dam, Iraq. Sci. Rep., 6.

Milly, P. C. D. 1994. Climate, interseasonal storage of soil-water, and the annual water-balance. Adv. Water Resour., 17, 19-24.

Milly, P. C. D., \& Dunne, K. A. 1994. Sensitivity of the global water cycle to the water holding capacity of land. J. Clim., 7, 506-526.

Mitchell, A. R. 1991. Soil surface shrinkage to estimate profile soil-water. Irrigation Sci., 12, 1-6.

Mitchell, A. R., \& Van Genuchten, M. Th. 1992. Shrinkage of bare and cultivated soil. Soil Sci. Soc. Am. J., 56, 1036-1042.

Monti Guarnieri, A., \& Tebaldini, S. 2008. On the Exploitation of Target Statistics for SAR Interferometry Applications. IEEE Trans. Geosci. Remote Sens., 46, 34363443.

Moreira, A., Krieger, G., Hajnsek, I., Papathanassiou, K., Younis, M., López-Dekker, P., Huber, S., Villano, M., Pardini, M., Eineder, M., Zan, F. De, \& Parizzi, A. 2015. Tandem-L: A Highly Innovative Bistatic SAR Mission for Global Observation of Dynamic Processes on the Earth's Surface. IEEE Trans. Geosci. Remote Sens. Mag., 3, 8-23.

Morishita, Y., \& Hanssen, R. F. 2015b. Deformation Parameter Estimation in Low Coherence Areas Using a Multisatellite InSAR Approach. IEEE Trans. Geosci. Remote Sens., 53, 4275-4283.

Morrison, K., Bennett, J. C., Nolan, M., \& Menon, R. 2011. Laboratory Measurement of the DInSAR Response to Spatiotemporal Variations in Soil Moisture. IEEE Trans. Geosci. Remote Sens., 49, 3815-3823.

Neely, H. L., Ackerson, J. P., Morgan, C. L. S., \& McInnes, K. J. 2014. Instrumentation to Measure Soil Subsidence and Water Content in a Single Borehole. Soil Sci. Soc. Am. J., 78, 1251-1257. 
Nelson, J. D., \& Miller, D. J. 1992. Expansive soils: Problems and Practice in Foundation and Pavement Engineering. New York: Wiley.

Nesti, G., Tarchi, D., Despan, D., Rudant, J. P., Bedidi, A., Borderies, P., \& Bachelier, E. 1998. Phase shift and decorrelation of radar signal related to soil moisture changes. In: Proc. 2nd International Workshop on Retrieval of Bio-Physical and Geo-Physical Parameters from SAR Data for Land Application. ESA, noordwijk, Netherlands.

Nijp, J. J. 2015. Fine scale ecohydrological processes in northern peatlands and their relevance for the carbon cycle. Ph.D. thesis, Wageningen University.

Nolan, M., \& Fatland, D. R. 2003. Penetration depth as a DInSAR observable and proxy for soil moisture. IEEE Trans. Geosci. Remote Sens., 41, 532-537.

Nolan, M., Fatland, D. R., \& Hinzman, L. 2003. DInSAR measurement of soil moisture. IEEE Trans. Geosci. Remote Sens., 41, 2802-2813.

Parker, J. C., Amos, D. F., \& Kaster, D. L. 1977. Evaluation of several methods of estimating soil volume change. Soil Sci. Soc. Am. J., 41, 1059-1064.

Parsons, L. R., \& Bandaranayake, W. M. 2009. Performance of a New Capacitance Soil Moisture Probe in a Sandy Soil. Soil Sci. Soc. Am. J., 73, 1378-1385.

Peng, X., \& Horn, R. 2007. Anisotropic shrinkage and swelling of some organic and inorganic soils. Eur. J. Soil Sci., 58, 98-107.

Peng, X., Zhang, Z. B., Gan, L., \& Yoshida, S. 2016. Linking Soil Shrinkage Behavior and Cracking in Two Paddy Soils as Affected by Wetting and Drying Cycles. Evaluation of several methods of estimating soil volume change, 80, 1145-1156.

Pietroniro, A., \& Leconte, R. 2000. A review of Canadian remote sensing applications in hydrology, 1995-1999. Hydrol. Process., 14, 1641-1666.

Rabus, B., Wehn, H., \& Nolan, M. 2010. The Importance of Soil Moisture and Soil Structure for InSAR Phase and Backscatter, as Determined by FDTD Modeling. IEEE Trans. Geosci. Remote Sens., 48, 2421-2429.

Randall, D. A., Wood, R. A., Bony, S., Colman, R., Fichefet, T., Fyfe, J., Kattsov, V., Pitman, A., Shukla, J., Srinivasan, J., et al. 2007. Climate models and their evaluation. In: Climate change 200\%: The physical science basis. Contribution of Working Group I to the Fourth Assessment Report of the IPCC (FAR). Cambridge University Press.

Reeves, J. A., Knight, R., Zebker, H. A., Schreuder, W. A., Agram, P. S., \& Lauknes, T. R. 2011. High quality InSAR data linked to seasonal change in hydraulic head for an agricultural area in the San Luis Valley, Colorado. Water Resour. Res., 47.

Reeves, J. A., Knight, R., Zebker, H. A., Kitanidis, P. K., \& Schreüder, W. A. 2014. Estimating temporal changes in hydraulic head using InSAR data in the San Luis Valley, Colorado. Water Resour. Res., 50, 4459-4473.

Richards, J. A. 2009. Remote sensing with imaging radar. Signals and communication technology. Berlin: Springer.

Rijniersce, K. 1983. A simulation model for physical soil ripening in the IJsselmeerpolders. Flevobericht No.233. Rijksdienst voor de IJsselmeerpolders, Lelystad.

Robinson, D. A., Campbell, C. S., Hopmans, J. W., Hornbuckle, B. K., Jones, S. B., 
Knight, R., Ogden, F., Selker, J., \& Wendroth, O. 2008. Soil moisture measurement for ecological and hydrological watershed-scale observatories: A review. Vadose Zone J., 7, 358-389.

Ross, P. J., \& Prebble, R. E. 1989. Non-destructive measurement of soil clod volume using hexane displacement. Aust. J. Soil Res., 27, 39-44.

Rott, H., \& Nagler, T. 2006. The contribution of radar interferometry to the assessment of landslide hazards. Adv. Space Res., 37(4), 710-719.

Rudant, J. P., Bedidi, A., Calonne, R., Massonnet, D., Nesti, G., \& Tarchi, D. 1996. Laboratory experiments for the interpretation of phase shift in SAR interferograms. In: Proc. FRINGE 1996 Workshop.

Sakaki, T., Limsuwat, A., Smits, K. M., \& Illangasekare, T. H. 2008. Empirical twopoint alpha-mixing model for calibrating the ECH2O EC-5 soil moisture sensor in sands. Water Resour. Res., 44, 526-531.

Samiei-Esfahany, S., Martins, J., Van Leijen, F. J., \& Hanssen, R. F. 2016. Phase Estimation for Distributed Scatterers in InSAR Stacks using Integer Least Squares Estimation. IEEE Trans. Geosci. Remote Sens., 54, 5671-5687.

Smith, L. C. 2002. Emerging applications of interferometric synthetic aperture radar (InSAR) in geomorphology and hydrology. Ann. Assoc. Am. Geogr., 92, 385-398.

Soil Survey Staff. 2010. Keys to Soil Taxonomy. 11 edn. USDA-Natural Resources Conservation Service, Washington, DC.

Spaans, K., Hreinsdóttir, S., Hooper, A., \& Ófeigsson, B. G. 2015. Crustal movements due to Iceland's shrinking ice caps mimic magma inflow signal at Katla volcano. Sci. Rep., 5.

Stewart, R. D., Abou Najm, M. R., Rupp, D. E., \& Selker, J. S. 2012. Measurement Tool for Dynamics of Soil Cracks. Vadose Zone J., 11(2).

Stewart, R. D., Rupp, D. E., Najm, M. R. Abou, \& Selker., J. S. 2016. A Unified Model for Soil Shrinkage, Subsidence, and Cracking. Vadose Zone J., 15.

Stirk, G. B. 1954. Some aspects of soil shrinkage and the effect of cracking upon water entry into the soil. Aust. J. Agr. Res., 5, 279-290.

Topp, G. C., Davis, J. L., \& Annan, A. P. 1980. Electromagnetic determination of soil water content: Measurements in coaxial transmission lines. Water Resour. Res., 16, 574-582.

Tucker, G. E., \& Bras, R. L. 2000. A stochastic approach to modeling the role of rainfall variability in drainage basin evolution. Water Resources Research, 36, 1953-1964.

Ulaby, F. T., Dubois, P. C., \& Van Zyl, J. 1996. Radar mapping of surface soil moisture. J. Hydrol., 184, 57-84.

Usai, S., \& Klees, R. 1999. SAR interferometry on a very long time scale: a study of the interferometric characteristics of man-made features. IEEE Trans. Geosci. Remote Sens., 37, 2118-2123.

Van der Kooij, M. W. A., Van Halsema, D., Groenewoud, W., Mets, G. J., Overgaauw, B, \& Visser, P. N. A. M. 1995. SAR Land Subsidence Monitoring. Delft: Beleidscommissie Remote Sensing. 
Van Heerwaarden, C. C., Vilá-Guerau de Arellano, Jordi, \& Teuling, Adriaan J. 2010. Land-atmosphere coupling explains the link between pan evaporation and actual evapotranspiration trends in a changing climate. Geophys. Res. Lett., 37.

Van Leijen, F. J. 2014. Persistent Scatterer Interferometry based on Geodetic estimation theory. Ph.D. thesis, Delft University of Technology.

Van Leijen, F. J., Ketelaar, V. B. H., Marinkovic, P. S., \& Hanssen, R. F. 2005. Persistent scatterer interferometry: Precision, reliability and integration. In: Proceedings of ISPRS Hannover Workshop.

Vereecken, H., Huisman, J. A., Bogena, H., Vanderborght, J., Vrugt, J. A., \& Hopmans, J. W. 2008. On the value of soil moisture measurements in vadose zone hydrology: A review. Water Resour. Res., 44.

Wagner, W., \& Pathe, C. 2005. Has SAR Failed in Soil Moisture Retrieval? In: Proc. of the 2004 Envisat 8 ERS Symposium.

Wagner, W., Bloschl, G., Pampaloni, P., Calvet, J. C., Bizzarri, B., Wigneron, J. P., \& Kerr, Y. 2007. Operational readiness of microwave remote sensing of soil moisture for hydrologic applications. Nord. Hydrol., 38, 1-20.

Wagner, W., Verhoest, N. E. C., Ludwig, R., \& Tedesco, M. 2009. Editorial 'Remote sensing in hydrological sciences'. Hydrol. Earth Syst. Sci., 13, 813-817.

Walker, J. P., Willgoose, G. R., \& Kalma, J. D. 2001. One-dimensional soil moisture profile retrieval by assimilation of near-surface observations: a comparison of retrieval algorithms. Adv. Water Resour., 24, 631-650.

Wdowinski, S., Amelung, F., Miralles-Wilhelm, F., Dixon, T. H., \& Carande, R. 2004. Space-based measurements of sheet-flow characteristics in the Everglades wetland, Florida. Geophys. Res. Lett., 31.

Wells, R. R., DiCarlo, D. A., Steenhuis, T. S., Parlange, J. Y., Römkens, M. J. M., \& Prasad, S. N. 2003. Infiltration and surface geometry features of a swelling soil following successive simulated rainstorms. Soil Sci. Soc. Am. J., 67.

Western, A. W., Grayson, R. B., \& Green, T. R. 1999. The Tarrawarra project: High resolution spatial measurement, modelling and analysis of soil moisture and hydrological response. Hydrol. Process., 13, 633-652.

Yang, J., Zhang, C., Li, X., Huang, Y., Fu, S., \& Acevedo, M. F. 2010. Integration of wireless sensor networks in environmental monitoring cyber infrastructure. Wirel. Netw., 16, 1091-1108.

Yule, D. F., \& Ritchie, J. T. 1980a. Soil Shrinkage Relationships of Texas Vertisols: 1. Small Cores. Soil Sci. Soc. Am. J., 44(6), 1285-1291.

Yule, D. F., \& Ritchie, J. T. 1980b. Soil Shrinkage Relationships of Texas Vertisols: 2. Large Cores. Soil Sci. Soc. Am. J., 44(6), 1291-1295.

Yuter, S. E., Kingsmill, D. E., Nance, L. B., \& Loeffler-Mang, M. 2006. Observations of precipitation size and fall speed characteristics within coexisting rain and wet snow. J. Appl. Meteorol. Clim., 45(10), 1450-1464.

Zebker, H. A., \& Goldstein, R. M. 1986. Topographic mapping from interferometric synthetic aperture radar observations. J. Geophys. Res.-Sol. Ea., 91(B5), 49934999. 
Zebker, H. A., \& Villasenor, J. 1992. Decorrelation in interferometric radar echoes. IEEE Trans. Geosci. Remote Sens., 30(5), 950-959.

Zebker, H. A., Rosen, P. A., Goldstein, R. M., Gabriel, A., \& Werner, C. L. 1994. On the derivation of coseismic displacement fields using differential radar interferometry: The Landers earthquake. J. Geophys. Res.-Sol. Ea., 99(B10), 19617-19634.

Zhang, R. B., Guo, J. J., Zhang, L., Zhang, Y., Wang, L. H., \& Wang, Q. 2011. A calibration method of detecting soil water content based on the information-sharing in wireless sensor network. Comput. Electron. Agr., 76(2), 161-168.

Zreda, M., Shuttleworth, W. J., Zeng, X., Zweck, C., Desilets, D., Franz, T., \& Rosolem, R. 2012. COSMOS: the COsmic-ray Soil Moisture Observing System. Hydrol. Earth Syst. Sci., 16(11), 4079-4099.

Zreda, Marek, Desilets, Darin, Ferré, T. P. A., \& Scott, R. L. 2008. Measuring soil moisture content non-invasively at intermediate spatial scale using cosmic-ray neutrons. Geophys. Res. Lett., 35(21).

Zwieback, S., Hensley, S., \& Hajnsek, I. 2015. Assessment of soil moisture effects on L-band radar interferometry. Remote Sens. Environ., 164, 77-89.

Zwieback, S., Liu, X., Antonova, S., Heim, B., Bartsch, A., Boike, J., \& Hajnsek, I. 2016. A Statistical Test of Phase Closure to Detect Influences on DInSAR Deformation Estimates Besides Displacements and Decorrelation Noise: Two Case Studies in High-Latitude Regions. IEEE Trans. Geosci. Remote Sens., 54(9), 5588-5601. 


\section{Bedankt! Thanks!}

Dit proefschrift is het resultaat van ruim acht jaar werk. In deze lange tijd zijn er veel personen direct of indirect betrokken geraakt bij het proces dat tot dit proefschrift geleid heeft. Het schrijven van dit proefschrift was onmogelijk geweest zonder de hulp en aanpassingsbereidheid van deze mensen. Ongetwijfeld vergeet ik een hoop mensen, maar toch wil ik een poging wagen om de belangrijkste van deze personen bij naam te noemen.

Tineke, wat was het fijn om jou als begeleidster te hebben! Je raakte betrokken door je kennis van bodemfysica, maar dit is niet de enige reden waarom ik je begeleiding zo gewaardeerd heb. Je liet me vaak mijn gang gaan, maar stond voor mij klaar met relativering en raad als ik vastliep in zijwegen, te kleine details, of de strijd tegen de klok. Misschien niet de meest efficiënte (al neem ik de complete verantwoordelijkheid voor de duur van het gehele proces op me) maar zeker een heel leerzame en prettige, persoonlijke, aanpak.

Sjoerd, ook jouw aanpak heb ik zeer gewaardeerd: je hebt Ger, Tineke en mij altijd veel vrijheid en vertrouwen gegeven, en het was prettig om zo nu en dan eens te rade te kunnen gaan bij iemand met een frisse kijk op ons werk.

Ramon, bedankt voor de kans om me te kunnen verdiepen in de wereld van de radar interferometrie, wat voor mij een grote uitdaging was. Ik wist me niet altijd raad met je zeer kritische blik en prikkelende vragen, maar het toont je gedrevenheid, waarvoor ik veel respect heb.

Ger, je vertrek uit Wageningen leverde wat complicaties op, en het wederzijds contact daarna had beter gekund, maar ik ben je dankbaar voor het initiëren van dit onderzoek, het vertrouwen in mij en de sturing in het eerste jaar. Tof dat je er vandaag bij bent!

Van geheel andere orde, maar niet minder belangrijk, was de hulp die ik kreeg bij mijn veldwerk in de Purmer. Dirk en de hele familie de Heer, jullie leken nooit een probleem te hebben met mijn activiteiten op jullie percelen en erf, waardoor ik op een heel prettige manier mijn werk heb kunnen doen. De gezellige koffiepauzes, waarin ik nog heel wat leerde over de akkerbouw, hielpen hier uiteraard ook bij! Achteraf kan ik wel zeggen dat de toestemming om na 2010 ook in het jaar 2011 nog door te meten op jullie percelen, beslissend is geweest voor het slagen van dit promotieonderzoek. Heel erg bedankt! Ook de families Knook en Stokman wil ik bedanken voor de geboden hulp, en ruimte en stroom. 
Bij het voorbereiden en uitvoeren van de veldcampagne en de metingen in het bodemfysisch lab, heb ik veel ondersteuning gehad van onmisbare technici. Harm, bedankt voor het vele sparren en testen. Hennie, zelfs in weekenden kon ik op je hulp rekenen, bedankt!

Al mijn collega's van de vakgroepen SEG, SLM en HWM in Wageningen bedankt voor alle leuke en leerzame momenten en voor de productieve en gezellige schrijfweekenden. Ype, het was heel prettig om een senior aio als kamergenoot en mentor te hebben die zo geïnteresseerd en hulpvaardig was als ik weer eens liep te stoeien met complexe getallen. Dieuwke, met jou een kantoor delen leverde een optimale mix van hard werken, goede muziek luisteren, en ontspanning.

Marjolein, sinds ons Vietnam avontuur hebben we een hechte band en onze gezamenlijke tijd als aio heeft hier nog eens flink aan bijgedragen. Bedankt voor de koffiepauzes, lunches, wandelingen, borrels, spelletjesavonden en alle andere activiteiten. Ik ben blij dat jij en Niko weer terug zijn in Nederland, zodat je vandaag mijn paranimf kan zijn: ik kan het me niet voorstellen om deze tijd af te sluiten zonder jouw aanwezigheid.

Het Vietnam avontuur leverde ook tijdens mijn hele aio-tijd nog een mooi project on the side: Anne, Marjolein en Roel, bedankt voor het leuke mangroveproject. Met het voltooien van dit proefschrift lijkt mijn wetenschappelijke carrière ten einde, al geef ik het nog een kans als het tot een voortzetting van ons mangrove onderzoek zou kunnen komen.

I will switch to English now as I owe a lot to my mostly non-Dutch colleagues from Delft. Especially Sami, Freek, and (in the early stages) Mahmut, thanks for helping me so patiently in finding my way around InSAR processing and interpretation. Sami, thanks so much for taking time to work on this project together. I could not have finished this without your expertise and help. To all colleagues: thanks for the warm welcome every time I showed up in Delft after long periods of absence. I hope I have been able to contribute to your projects the slightest bit, with some knowledge about soils and soil moisture.

Mijn werkgevers van de afgelopen vier jaar, Eijkelkamp Soil \& Water en Ingenieursbureau Land, wil ik bedanken voor het vertrouwen en de ruimte die ik heb gekregen om dit proefschrift af te maken.

Ik heb een hele hoop vrienden die ik moet bedanken, voor hun hulp of gewoon voor hun aanwezigheid.

Henk, mede-Achterhoeker, jaargenoot, AkCie-man, GPG'er, maar bovenal enorm loyale en goede vriend; wat tof dat je mijn paranimf wil en kan zijn. Ik waardeer de moeite die jij en Anne hiervoor hebben genomen enorm!

Miranda, bedankt dat je er altijd voor mij (en Sara, en de katten) bent. Ik laat het misschien niet altijd merken, maar het is geweldig om je in de buurt te hebben! 
Bedankt voor je onvoorwaardelijke vriendschap en de LaTeX hulp.

Chiel, Ruud, Anneke en alle andere fietsers: bedankt voor de fijne tochtjes en andere activiteiten.

Mijn homies (4 leif), bedankt voor de geweldige tijd samen in VGH lang geleden, welke toch nog deels overlapte met mijn aio-schap. Straks sambuca in The Doctor?

Al lang voordat ik het woord 'proefschrift' überhaupt kende, waren de kerels van de Eibergse Alliantie er al om een prachtige vriendschap mee te delen. Dirk, Janwillem, Sten, Arend, Daniël, Jordey, Dennis en Bart, bedankt voor alle jaren door dik en dun. Het is allemaal misschien wat makker geworden, maar elke keer als weer samen komen voelt het ouderwets goed. En één keer in het jaar een weekend helemaal los met vliegen op de lamp en handen op de tafel is daar het beste bewijs van. $\mathrm{Nu}$ dit proefschrift af is, is mijn aanwezigheid de komende jaren gegarandeerd. Bedankt voor het geduld, de afleiding, de uitlaatklep, kritische vragen en mooie verhalen. Janwillem (en Danielle), ook bedankt voor het regelmatig bieden van een slaapplaats in Den Haag.

Nog zo'n mooie groep vrienden is het Grutte Pier Genootschap. We zijn allemaal wel heel serieus geworden en de borrels vinden helaas vaak plaats in klein gezelschap, maar het is altijd weer een mooi weerzien. De GPG-weekendjes zullen wel weer aantrekken als jullie allemaal gaan trouwen, en we samen white Russians kunnen bouwen. Auto's huren laat ik dan aan een ander over.

Just before I started my PhD I began playing Ultimate frisbee, but I could not have imagined how much this would offer me. I'd like to thank everyone who I've shared practices, games, and parties with, which provided great fun, goals, and friendships. Especially my team mates from Dutch Mixed 2012, UFO Open 2014, Dutch Open 2015, En de rest. But when it comes to frisbee, most of all I have to thank all my former and current team mates at WAF. Not only did WAF, and everyone involved in the club, provide great pleasure and distraction, but it did also offer me the chance to develop as player, coach, and person. Thanks to all players who have been truly great at enduring my mood swings on the field in times of stress. I feel grateful for your friendship, patience, feedback, and respect, some aspects for which I truly love WAF.

Pap en mam, het kan niet anders dan dat jullie zo nu en dan aan mijn keuzes hebben getwijfeld de afgelopen acht jaar, maar ik heb me altijd door jullie gesteund gevoeld. Ik kon altijd bij jullie aankloppen voor hulp, waarvan het oppassen op Lisanne het afgelopen jaar slechts een voorbeeld is. Bedankt dat jullie er voor mij waren, zonder het onderwerp proefschrift al te vaak aan te kaarten. Jullie zijn geweldige ouders en grootouders! Elske, het was fijn dat je Sara en mij regelmatig kwam bezoeken, aangezien wij door mijn toedoen alle moeite hadden om fatsoenlijk een bezoekje aan jou te plannen. Gijs, je strenge woorden waren soms confronterend, maar ook motiverend en leerzaam. Mooi dat we nu samen kunnen werken aan interessante projecten! En Maaike, zullen de bibliotheek medewerkers zich ooit afgevraagd hebben waar een hydroloog toch al die artikelen over de culturele geschiedenis van Indonesië voor nodig heeft? Veel succes met de laatste loodjes! 
Lieve Sara, ik heb zo ontzettend veel tijd van je gestolen en zo veel misbruik gemaakt van je onvoorwaardelijke liefde. Je hebt me de ruimte gegeven om dit slepende project tot een goed einde te brengen, zonder eisen te stellen en zonder me beperkingen op te leggen. Ik denk niet dat ik jouw investering in mij, mijn trots en eigenwaarde ooit terug kan betalen. Bedankt voor alles wat je voor me hebt gedaan en nog altijd doet. Ik kijk uit naar de toekomst met jou en Lisanne! 


\section{List of publications}

\section{Peer-reviewed articles}

Te Brake, B., Van der Ploeg, M.J., and De Rooij, G.H.: Water storage change estimation from in situ shrinkage measurements of clay soils. Hydrology and Earth System Sciences, 17, 1933-1949, 2013. doi:10.5194/hess-17-1933-2013.

Te Brake, B., Hanssen, R.F., Van der Ploeg, M.J., and De Rooij, G.H.: Satellitebased radar interferometry to estimate large-scale soil water depletion from clay shrinkage: possibilities and limitations. Vadose Zone Journal, vol. 12, num. 3, 2013. doi:10.2136/vzj2012.0098.

Van Loon, A.F., Te Brake B., Van Huijgevoort M.H.J., and Dijksma, R.: Hydrological Classification, a Practical Tool for Mangrove Restoration. PLoS ONE 11(3): e0150302, 2016. doi:10.1371/journal.pone.0150302.

\section{Book chapters}

Dijksma, R., Van Loon, A.F., Van Mensvoort, M.E.F., Van Huijgevoort, M.H.J., and Te Brake, B.: An Extended Hydrological Classification for Mangrove Rehabilitation Projects: A Case Study in Vietnam. In: Tropical Deltas and Coastal Zones: Food Production, Communities and Environment at the Land-Water Interface (eds. C.T. Hoanh et al.), CAB International, 2010, ISBN: 978184593 6181. 
The research described in this thesis was financially supported by Netherlands Space Office (NSO) under project GO-AO/12, entitled 'Measuring land elevation changes of clays by radar interferometry to quantify soil water storage change'.

Financial support from Wageningen University for printing this thesis is gratefully acknowledged.

On the cover: shrinkage crack in clayey soil in the Purmer (greyscale, photo by author), with parts of a TerraSAR-X interferogram over Noord-Holland (data provided by the German Aerospace Center). Cover design by Sara Niermeijer. 


\section{SENSE}

Netherlands Research School for the

Socio-Economic and Natural Sciences of the Environment

\section{I P L O M A}

For specialised PhD training

The Netherlands Research School for the Socio-Economic and Natural Sciences of the Environment (SENSE) declares that

\section{Bram te Brake}

born on 21 January 1984 in Eibergen, the Netherlands

has successfully fulfilled all requirements of the Educational Programme of SENSE.

Wageningen, 17 May 2017

the Chairman of the SENSE board

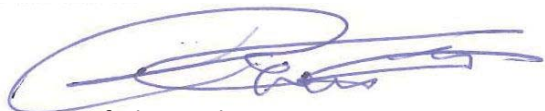

Prof. dr. Huub Rijnaarts the SENSE Director of Education

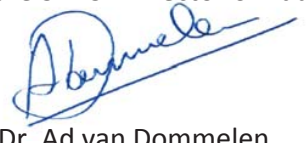

Dr. Ad van Dommelen

The SENSE Research School has been accredited by the Royal Netherlands Academy of Arts and Sciences (KNAW)

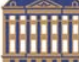

$\begin{array}{llllllllllllllllllllll}K & O & N & I & N & K & L & I & J & K & E & N & E & D & E & R & L & A & N & D & S & E\end{array}$ $\begin{array}{lllllllllllllllllllllllll}\text { A } & K & \text { A } & \text { D } & \text { E } & M & \text { I } & \text { E } & \text { V } & \text { A } & N & \text { W } & \text { E } & T & \text { E } & N & \text { S } & C & H & \text { A } & P & P & \text { E } & N\end{array}$ 


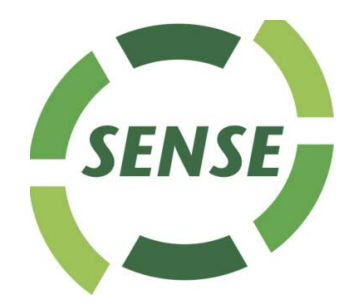

The SENSE Research School declares that Mr Bram te Brake has successfully fulfilled all requirements of the Educational PhD Programme of SENSE with a work load of 33.9 EC, including the following activities:

\section{SENSE PhD Courses}

- Environmental research in context (2010)

- Research in context activity: 'Co-organising WIMEK/SENSE symposium on: Modelling \& observing earth systems compartments' (2011)

\section{Other PhD and Advanced MSc Courses}

- Uncertainty Modelling and Analysis, Wageningen University (2010)

- Mobilising your-scientific-network, Wageningen University (2010)

- Techniques for Writing a Scientific Paper, Wageningen University (2011)

- Project and Time Management, Wageningen University (2012)

\section{External training}

- Training in processing and interpretation of satellite radar data with Doris software, Delft University of Technology (2010)

- Nest InSAR course, European Space Agency (2011)

\section{Management and Didactic Skills Training}

- Supervising two BSc students with thesis entitled 'Potential use of swell and shrinkage measurements of clays in hydrology' (2011) and 'Water storage change in clay soils derived from surface elevation changes and soil shrinkage characteristics' (2013)

\section{Oral Presentations}

- Pesticide leaching FOCUS scenarios if only dissolved pesticides degrade: re-assessing the importance of soil water flow. General Assembly European Geosciences Union, 19-24 April 2009, Vienna, Austria

- Measuring land elevation changes of clays by InSAR to quantify water storage change. AgriSAR 2009 Experimenters Meeting, ESA-ESTEC, 11 June 2009, Noordwijk, the Netherlands

- Upscaling soil water storage change in clay areas using radar interferometry. General Assembly European Geosciences Union, 3-8 April 2011, Vienna, Austria

- Linking InSAR patterns in polder areas to hydrological phenomena. WIMEK-SENSE symposium 'Water and energy cycles at multiple scales', Wageningen, 1 March 2012, Wageningen, the Netherlands

SENSE Coordinator PhD Education 